\title{
COMPUTATIONAL FLUID DYNAMICS APPLIED TO CHEMICAL REACTION ENGINEERING
}

\author{
J. A. M. Kuipers and W. P. M. van Swaaij \\ Department of Chemical Engineering \\ Twente University of Technology \\ 7500 AE Enschede, The Netherlands
}

$\begin{array}{lc}\text { I. Introduction } & 227 \\ \text { II. Traditional Approaches Followed within Chemical Reaction } & \\ \text { Engineering } & 228 \\ \text { A. Role and Types of Modeling in Chemical Engineering } & 232 \\ \text { B. Relation with Experimental Work } & 233 \\ \text { C. CFD in Chemical Engineering Education } & 234 \\ \text { III. Computational Fluid Dynamics } & 234 \\ \text { A. Definition and Theoretical Framework } & 236 \\ \text { B. Numerical Techniques } & 244 \\ \text { C. Existing Software Packages } & 251 \\ \text { IV. Application to Chemical Reaction Engineering } & 253 \\ \text { A. Single-Phase Systems } & 254 \\ \text { B. Multiphase Systems } & 265 \\ \text { C. State of the Art of CFD in Chemical Reaction Engineering } & 280 \\ \text { V. Experimental Validation } & 282 \\ \text { VI. Selected Applications of CFD Work Conducted at Twente } & \\ \text { University } & 287 \\ \text { A. Two-Fluid Simulation of Gas Fluidized Beds } & 287 \\ \text { B. Discrete Particle Simulation of Gas Fluidized Beds } & 291 \\ \text { C. Circulating Fluidized Beds } & 296 \\ \text { D. Bubble Columns } & 298 \\ \text { E. Modeling of a Laminar Entrained Flow Reactor } & 311 \\ \text { VII. Conclusion } & 313 \\ \text { Nomenclature } & 316 \\ \text { References } & 319\end{array}$

\section{Introduction}

In the last few decades computational fluid dynamics has become a very powerful and versatile tool for the analysis and solution of problems that are of considerable interest to the chemical engineer, despite the fact that CFD has not yet 227 
reached its full potential. The chemical engineering discipline has developed many valuable semiempirical strategies to solve problems of practical interest. Prior to expanding on the nature of CFD and its current possibilities and limitations, a brief outline on these strategies will be presented.

\section{Traditional Approaches Followed within Chemical Reaction Engineering}

In the process technology raw materials are converted into desired products via chemical and physical processes. These products are often intermediates that are subsequently converted in other production processes. This general scheme (see Fig. 1) from raw materials like minerals, crude oil, natural gas, agricultural products, etc., via preprocessing and purification is very common. A process engineer developing, designing, or optimizing a process has to deal with many disciplines varying from chemistry or biochemistry to economy. His or her ultimate aim is to produce a valuable product in a safe way at acceptable cost and burden to the environment. Also time is a scarce quantity because he or she has to take the changing market and competitors into account. The traditional chemical engineering sciences like transport phenomena and chemical reaction engineering play an essential role in achieving the aforementioned aims. In these sciences elements from physics (like transport theories, fluid dynamics, and thermodynamics), chemistry (kinetics, catalysis), and mathematics have been integrated to form dedicated tools to tackle extremely complicated problems that come up in studying and developing processes.

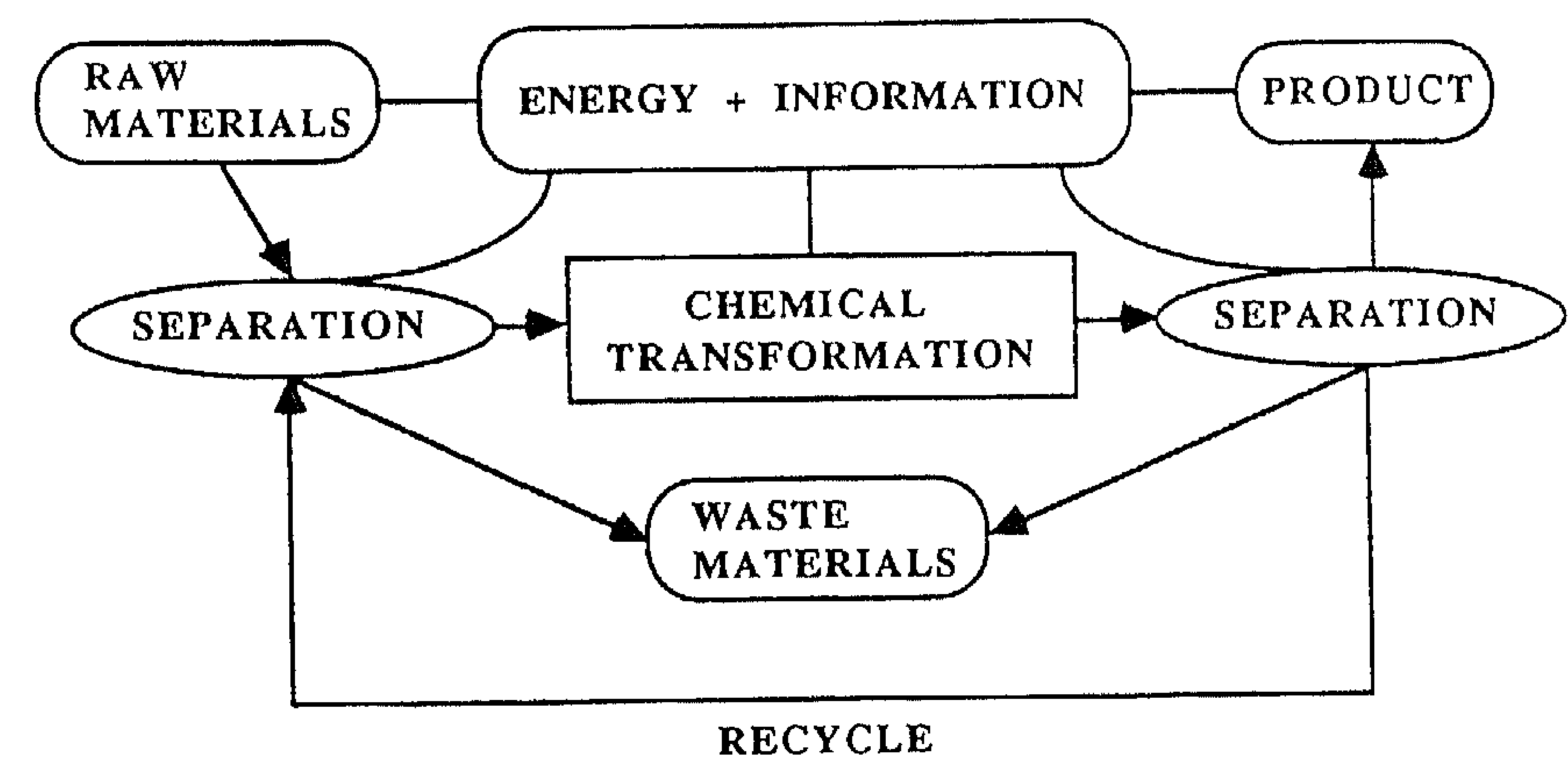

FIG. 1. Schematic representation of a production scheme typically encountered in process technology. 
A new emerging tool is a combination of fluid dynamics and numerical mathematics backed up by the immense growth of computer power: computational fluid dynamics (CFD). It is affecting the chemical engineering sciences and the art of the chemical engineer in a profound way. We also think that a special branch of CFD will originate from the confrontation with chemical engineering science. This is because of special demands in the applications, the applicationoriented attitude of the chemical engineer, and the way the knowledge has been organized traditionally.

Originally, technical scientific knowledge was arranged around the individual process steps: distillation, absorption, extraction, crystallization, heat exchange. mass exchange, etc.; the so-called "unit operations" $( \pm 1920)$. For a short period of time also the notion of unit processes was used for chemical process steps such as chlorination, oxydation, hydrogenation, etc. However, because of lack of coherence this never became a big success. Specifically after the second world war it became increasingly clear that the different unit operations could be strongly unified by combining the principle of microbalance formulation with laws of linear transport of mass, momentum, and energy while adding elements of technical fluid dynamics and radiative heat transfer. This culminated in the textbook Transport Phenomena by Bird. Stewart, and Lightfoot (1960), which is still a standard for chemical engineers. Chemical reactions often form a considerable complicition in process description and modeling. Integration of chemical reaction kinetics and thermodynamics in transport phenomena resulted in the discipline of chemical reaction engineering. The first textbooks on chemical reaction engineering appeared in the early 1960s (Levenspiel, 1962). Of course, these sciences have grown since then and the widespread use of computing facilities changed their nature allowing, amongst others, the chemical engineer to use large databases, advanced design and optimization methods, and complex and extended models that were previously difficult to handle. The increased use of the Maxwell-Stefan transport model instead of the traditional Fickian model represents an example of this development.

As mentioned earlier, in the past many powerful tools with a strong empirical base had been devised by chemical engineers to (approximately) solve extremely complicated problems encountered in chemical reaction engineering such as the design of process equipment (including chemical reactors) involving nonideal flow (see Fig. 2; Levenspiel, 1962). Very often the two idealized flow patterns, that is, plug flow and mixed flow, do not occur in reality but nevertheless these concepts have proven valuable when dealing with the design of chemical reactors because the conversion obtained in these two extreme cases provides the boundaries for the conversion in a chemical reactor in which nonideal flow prevails. Levenspiel (1962) stated that "If we know precisely what is happening within the vessel, thus if we have a complete velocity distribution map for the ffuid, then we are able to predict the behavior of a vessel as a reactor. Though fine in principle, the attendant 

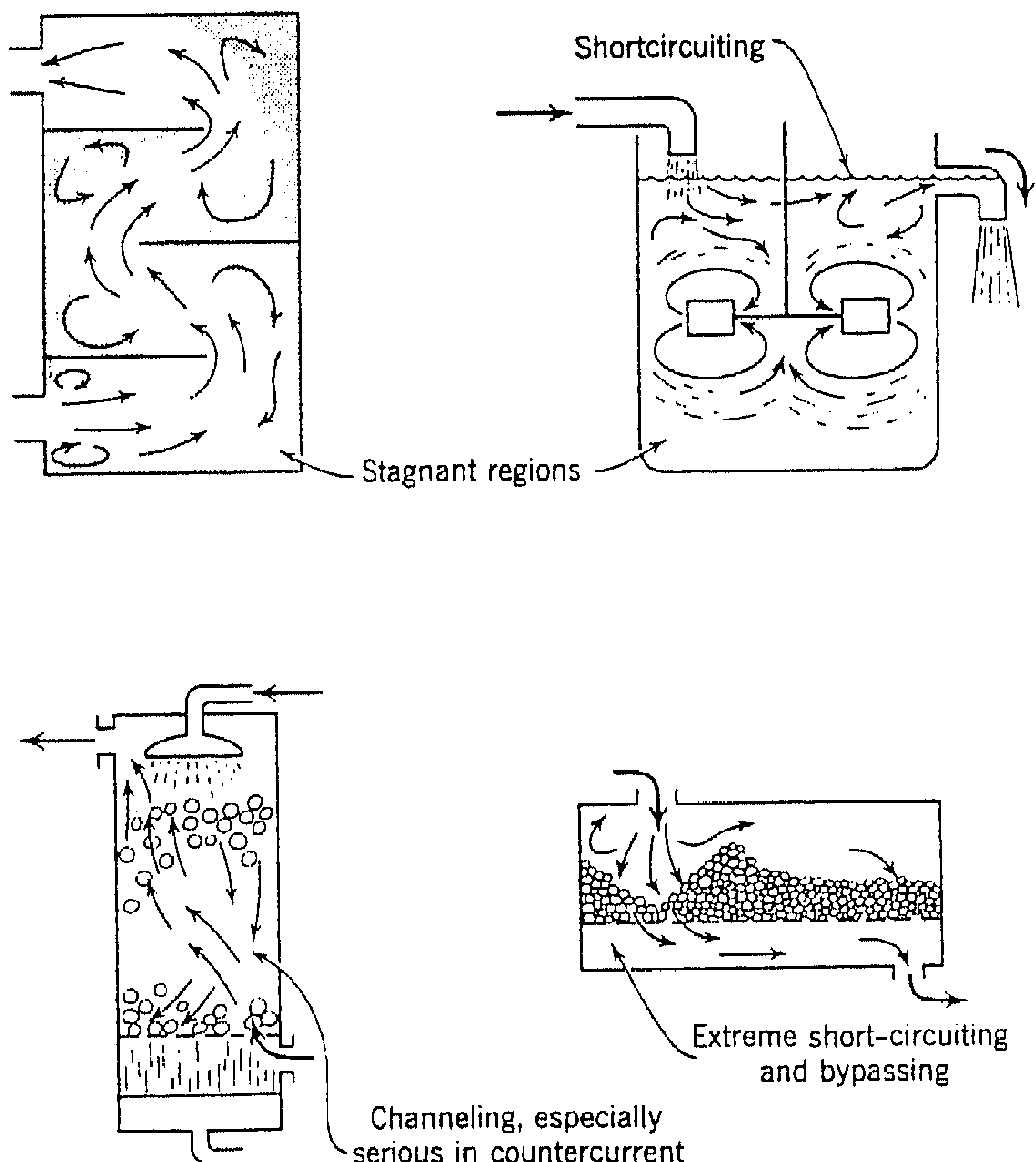

Channeling, especially serious in countercurrent

two-phase operations

Fic. 2. Some typical examples of nonideal flow patterns that can occur in process equipment. (From Levenspiel. O.. "Chemical Reaction Engineering." John Wiley \& Sons. New York, 196?. Reprinted by permission of John Wiley \& Sons. Inc.)

complexities make it impractical to use this approach." This situation has clearly changed because nowadays the "complete velocity distribution map" can in principle be obtained by computing the velocity distribution in the system of interest.

A well-known traditional approach adopted in chemical engineering to circumvent the intrinsic difficulties in obtaining the "complete velocity distribution map" is the characterization of nonideal flow patterns by means of residence time distribution (RTD) experiments where typically the response of a piece of process equipment is measured due to a disturbance of the inlet concentration of a tracer. From the measured response of the system (i.e., the concentration of the tracer measured in the outlet stream of the relevant piece of process equipment) the differential residence time distribution $E(t)$ can be obtained where $E(t) d t$ represents 
the fraction $d F(t)$ of the volume elements with a residence time between $t$ and $t+d t$ :

$$
d F(t)=E(t) d t
$$

The fraction of the fluid elements with a residence time of less than $t$ is given by the cumulative residence distribution function $F(t)$ given by:

$$
F(t)=\int_{0}^{t} E(t) d t
$$

For a continuous reactor with a nonideal flow pattern, characterized by the differential residence time distribution $E(t)$, the following expression holds for the conversion $\xi_{\text {nonideal }}$, which is attained in case complete segregation of all fluid elements passing through the reactor can be assumed:

$$
\xi_{\text {nonideal }}=\int_{t_{\text {min }}}^{t_{\text {max }}} \xi_{\text {batch }}(t) E(t) d t .
$$

where $\xi_{\text {batth }}(t)$ represents the conversion attained in a batch reactor with batch time $t$. Of course, in reality a certain degree of mixing between the fluid elements passing through the reactor occurs with simultaneous chemical transformation and therefore information on the macromixing patterns is generally not sufficient to enable accurate prediction of the extent of chemical conversion in a reactor possessing a nonideal flow pattern. This mixing phenomenon necessitates the use of a micromixing model to account for the finite rate with which segregated fluid elements eventually achieve mixing at the molecular level (i.e., segregation decay) to permit the occurrence of a chemical transformation.

Due to the advent of CFD the aforementioned approach can still be followed but now the $E(t)$ and $F(t)$ functions can in principle be computed from the computed velocity distribution. Alternatively, the species conservation equations can be solved simultaneously with the fluid fow equations and thereby the extent of chemical conversion can also be obtained directly without invoking the concept of residence time distributions.

The strong demand for data reduction also applies to results of CFD. Moreover chemical engineers have a sound distrust of, based on a lot of experience. calculated results that have not been validated with experimental data. If we compare the traditional chemical engineering approach to the pure computational fluid dynamics-based procedure several advantages and disadvantages become apparent and at least for a long period of time both approaches have to (and probably will) merge to obtain an optimal result. However, more and more the traditional empirical and phenomenological models are being replaced by more fundamental descriptions, which are based on the full microbalances for mass, momentum. and 
(thermal) energy. In the next section a brief discussion is presented of the role and types of modeling encountered in chemical engineering.

\section{A. Role and Types of Modeling in Chemical Engineering}

The widespread availability of fast computing facilities and the rapid advance of powerful numerical techniques and software offer the possibility of numerical simulation of many processes of interest in chemical engineering science and the process industry. Problems often encountered in process operations can be described by balance equations for conservation of mass, momentum, and (thermal) energy in combination with transport equations for chemical conversion or phase transition. Because the equations are in most cases coupled and nonlinear, the problem is complex. Moreover, transport rates are dependent on local fluid properties, which are often themselves a function of process variables. Therefore, only in the simplest cases can analytical solutions be found and numerical solution procedures are welcomed. CFD has been applied successfully in analyzing complex single-phase laminar flow problems. Here the accuracy of the simulations is so high that validation experiments are often considered unnecessary. This is certainly not the case for single-phase turbulent fiow problems in complex geometries, although considerable progress has been made here in recent years in turbulence modeling.

Several computer codes are now commercially available (see Section III.C) that are applicable for laminar and turbulent single-phase fluid flow problems. They show variable success in different applications. For two-phase flow the situation is still more complicated and detailed microbalance modeling is still in its infancy. Before we discuss these types of models in more detail, we first consider their advantages and disadvantages in comparison with other type of models. Specifically a comparison between detailed microbalance models (i.e., fluid flow equation-based microbalance models) and global system models (i.e.. models that are not based on fluid flow equations) is made: Table I gives a short overview. A similar table has been presented by Villermaux (1996). The arguments given in Table I are global ones, because the boundaries between the different models are not clear-cut. Moreover, a wide range of model types is possible that shows an important overlap in properties.

The most powerful property of the detailed microbalance models, especially in combination with visualization techniques, is the a priori prediction of (observable) macroscale phenomena. This can be particularly helpful in reducing the required experimental effort. Important problems are the amount of detailed information required for the microscale transport equations and the large programming and computational efforts required to solve specific problems. Nevertheless, these types of models, by generating insight in the micro- and 
TABLE I

Comparison of Detalled Microbalance Models with Global System Models

\begin{tabular}{|c|c|}
\hline $\begin{array}{c}\text { Detailed Microbalance Models } \\
\text { Advantage }\end{array}$ & $\begin{array}{c}\text { Global System Models } \\
\text { Advantage }\end{array}$ \\
\hline More exact solution available & $\begin{array}{l}\text { Simple models and simple solutions facilitate } \\
\text { understanding }\end{array}$ \\
\hline Phenomena follow trom calculations a priori & $\begin{array}{l}\text { Can be adapted to the detail of information } \\
\text { required }\end{array}$ \\
\hline $\begin{array}{l}\text { Formal balance equations can be written } \\
\text { straightforwardly (for single phase systems) }\end{array}$ & $\begin{array}{l}\text { Limited calculation capacity often sufficient } \\
\text { After adjustment of parameters accurate }\end{array}$ \\
\hline Processes can be visualized & macroscale behavior prediction \\
\hline Disadvantage & Disadvantage \\
\hline $\begin{array}{l}\text { Detailed knowledge required about the } \\
\text { elementary process }\end{array}$ & $\begin{array}{l}\text { Lot of a priori knowledge together with } \\
\text { imaginative power required }\end{array}$ \\
\hline $\begin{array}{l}\text { Massive data production. thus data } \\
\text { reduction required }\end{array}$ & $\begin{array}{l}\text { Experimental validation and adjustment of } \\
\text { parameters necessary }\end{array}$ \\
\hline Large calculation capacity required & Meaning of parameters sometimes unclear \\
\hline $\begin{array}{l}\text { Difficult to generalize. Each specilic } \\
\text { problem requires additional } \\
\text { (computational) effort }\end{array}$ & due to lumping \\
\hline $\begin{array}{l}\text { Macroscopic behavior not always } \\
\text { accurately predicted }\end{array}$ & \\
\hline
\end{tabular}

mesoscale mechanisms, can play an important role in the preparation of experimental programs and in innovations to improve existing processes.

\section{B. Relation With EXPERIMENTAL WORK}

Despite its great potential, in the near future CFD will not completely replace experimental work or standard approaches currently used by the chemical engineering community. In this connection it is even not sure that CFD is guaranteed to succeed or even be an approach that will lead to improved results in comparison with standard approaches. For single-phase turbulent flows and especially for multiphase flows, it is imperative that the results of CFD analysis somehow be compared with experimental data in order to assess the validity of the physical models and the computational algorithms. In this connection we should mention that only computational results that possess invariance with respect to spatial and temporal discretization should be confronted with experimental data. A CFD model usually gives very detailed information on the temporal and spatial variation of many key quantities (i.e., velocity components, phase volume fractions, temperatures, species concentrations, turbulence parameters), which leads to in- 
creasing demands for experimental methods. In this way CFD is fruitful because it can lead to the development of new techniques to measure quantities that previously have not been considered in detail.

\section{CFD in Chemical Engineering Education}

Due to the rapid advances in CFD and the potential it provides to analyze, on a fundamental basis, systems of considerable interest to the chemical engineer it can be anticipated that the importance of CFD as a "workhorse" for the chemical engineering community will rapidly increase in the near future. This development implies that the chemical engineer working with CFD will need a good knowledge in a large number of disciplines. including physics, chemistry, thermodynamics, materials science, fluid dynamics, chemical reaction engineering, and numerical and experimental methods. Thus broader university education is important and the ability to participate in interdisciplinary research teams is considered very important to meet the demands of an integrated approach combining experimental and theoretical methods with numerical simulation techniques. In this connection it is considered of crucial importance for (introductory) CFD courses to be implemented in the curricula of technical universities at (preferably) the undergraduate level. As far as the authors know most technical universities do not yet offer such courses.

\section{Computational Fluid Dynamics}

First we give a brief introduction to the historical development of the science of fluid mechanics, and subsequently the development and present areas of application of CFD are highlighted.

The equations that form the theoretical foundation for the whole science of fluid mechanics were derived more than one century ago by Navier (1827) and Poisson (1831) on the basis of molecular hypotheses. Later the same equations were derived by de Saint Venant (1843) and Stokes (1845) without using such hypotheses. These equations are commonly referred to as the Navier-Stokes equaltions. Despite the fact that these equations have been known of for more than a century, no general analytical solution of the Navier-Stokes equations is known. This state of the art is due to the complex mathematical (i.e., nonlinearity) nature of these equations.

Toward the end of the nineteenth century the science of fluid mechanics began to develop in two branches, namely theoretical hydrodynamics and hydraulics. The first branch evolved from Euler's equations of motion for a frictionless, non- 
viscous fluid, whereas the development of the second branch was driven by the rapid progress in technology were engineers, faced with the solution of practical problems for which the "classical" science of theoretical hydrodynamics had no answers, developed their own highly empirical science of hydraulics. At the beginning of this century Prandtl showed how the unification of the aforementioned divergent branches of fluid mechanics could be obtained. He showed both experimentally and theoretically that the neglect of the viscous forces, which are indeed very small compared to the remaining forces for the two most important fluids encountered in practice (water and air), leads to incorrect results in thin fluid regions near solid walls. In this region, the boundary layer, viscous forces are very important and therefore should be taken into account.

During the first half of this century a spectacular development in the boundary layer theory took place that was driven mainly by the needs of the aerodynamics community. Most of the initial developments involved approximate analytical solution or transformation and subsequent numerical integration of the relevant fluid flow equations (Schlichting, 1975). Until 1960 CFD was virtually absent in the aerodynamics discipline but gradually the aforementioned solution procedures were replaced by CFD-based approaches where the full conservation equations were solved. However, until 1970 the storage capacity and the speed of digital computers were not sufficient to enable efficient calculation of full three-dimensional flow fields around airplanes. This situation has by now definitely changed since a number of computer programs for the calculation of threedimensional flow fields around airplanes have become industry standards. resulting in their use as a tool in the design process. It can be anticipated that for the (future) design of hypersonic aircraft, where the ground test facilities - wind tunnels-do not exist to cover all relevant flow regimes, CFD will be the principal workhorse for the actual design. An interesting review of CFD applications in aeronautics has been presented by Jameson (1988), whereas the book of Anderson (1995) gives an excellent introduction to the basics of CFD and its applications in, amongst others, aeronautical engineering.

Although the initial development of the CFD discipline was driven by the aerodynamics community, nowadays CFD is truly interdisciplinary since its cuts across all disciplines where the analysis of fluid flow and associated phenomena is of importance. For example, CFD has found application in the automobile industries to study both the internal flow in combustion engines (Griffin et al., 1978) and the external flow (Shaw, 1988, Matsunaga et al., 1992). Also in civil engineering CFD has found application in the study of problems involving flow dynamics of rivers, lakes, and estuaries and external flow around buildings. In environmental engineering CFD has been used to analyze the complex flow patterns that exist in various types of furnaces (Bai and Fuchs, 1992). Here the CFD approach has proven useful to optimize furnace performance (i.e., improved thermal efficiency and reduction of emission of pollutants). CFD has also been applied to 
calculate air currents throughout buildings in order to arrive at improved designs of (natural) ventilation systems (Alamdari et al., 1991).

In industrial manufacturing applications a myriad of applications exist of which the modeling of chemical vapor deposition reactors in the semiconductor industries (Steijsiger et al., 1992) and the modeling of the casting process of liquid metals (Mampaey and Xu, 1992) can be mentioned as examples. For further details on CFD applications in various industrial manufacturing processes the reader is referred to previous review's (Colenbrander, 1991; Trambouze, 1993; Johansen and Kolbeinsen, 1996). Colenbrander has prepared a review on CFD applications in the petrochemical industries with specific emphasis on CFD applications and related experimental work carried out in the Shell Group laboratories. The application of CFD to chemical reaction engineering has been reviewed by Trambouze (1993). During the last two decades CFD has also become a powerful tool for analyzing and designing metallurgical processes. Johansen and Kolbeinsen (1996) have recently prepared a review on this subject, where CFD applications at SINTEF Materials and Technology were highlighted. Finally, Harris et al. (1995) have recently presented a review on the application of CFD in chemical reaction engineering (CRE) with emphasis on single-phase flow applications. Before the application of CFD to CRE is discussed, a brief outline of CFD and its theoretical framework are first presented.

\section{A. Definition and Theoretical Framework}

Computational fluid dynamics involves the analysis of fluid flow and related phenomena such as heat and/or mass transfer, mixing, and chemical reaction using numerical solution methods. Usually the domain of interest is divided into a large number of control volumes (or computational cells or elements) which have a relatively small size in comparison with the macroscopic volume of the domain of interest. For each control volume a discrete representation of the relevant conservation equations is made after which an iterative solution procedure is invoked to obtain the solution of the nonlinear equations. Due to the advent of high-speed digital computers and the availability of powerful numerical algorithms the CFD approach has become feasible. CFD can be seen as a hybrid branch of mechanics and mathematics. CFD is based on the conservation laws for mass, momentum, and (thermal) energy, which can be expressed as follows:

1. Mass is conserved.

2. Newton's second law: $\bar{F}=m \cdot \bar{a}$.

3. Energy is conserved.

Subsequently, the theoretical foundation is briefly explained where the authors have chosen to make a distinction between single-phase systems and multiphase systems. 


\section{Single-Phase Systems}

For single-phase systems involving laminar flows the conservation equations are firmly established. The mass and momentum conservation equations are respectively given by (Bird et al., (1960):

$$
\begin{gathered}
\frac{\partial}{\partial t} \rho+(\nabla \cdot \rho \bar{u})=0 \\
\frac{\partial}{\partial t} \rho \bar{u}+(\nabla \cdot \rho \bar{u} u)=-\nabla p-(\nabla \cdot \tau)+\rho \bar{g}
\end{gathered}
$$

where $\rho, \bar{u}, p$, and $\tau$, respectively, represent the fluid density, fluid velocity, pressure, and viscous stress tensor. For nonisothermal systems the mass and momentum conservation equations, Eq. (4) and (5), have to be supplemented with an energy equation for which either the total (i.e., the sum of internal energy and mechanical energy) or thermal energy equation can be used. Since in many chemical engineering applications (especially those dealing with chemical reactors) the mechanical energy changes are relatively small compared to the changes in thermal energy, the thermal energy equation is commonly used. For systems involving chemical transformations, conservation equations for all species $i$ involved have to be added. The thermal energy equation and the conservation equation for species $i$ are respectively given by:

$$
\frac{\partial}{\partial t}(\rho e)+(\nabla \cdot \rho e \bar{u})=-(\nabla \cdot \bar{q})-p(\nabla \cdot \bar{u})+(-\tau: \nabla \bar{u})+S_{\mathrm{h}}
$$

and

$$
\frac{\partial}{\partial t}\left(\rho \omega_{i}\right)+\left(\nabla \cdot \rho \bar{u} \omega_{i}\right)=-\left(\nabla \cdot \bar{J}_{i}\right)+S_{i}
$$

In Eqs. (6) and (7) $e$ represents the internal energy per unit mas, $\bar{q}$ the heat flux vector due to molecular transport, $S_{\mathrm{h}}$ the volumetric heat production rate, $\omega_{i}$ the mass fraction of species $i, \vec{J}_{i}$ the mass flux vector of species $i$ due to molecular transport, and $S_{i}$ the net production rate of species $i$ per unit volume. In many chemical engineering applications the viscous dissipation term $(-\tau: \nabla \bar{u})$ appearing in Eq. (6) can safely be neglected. For closure of the above set of equations. an equation of state for the density $\rho$ and constitutive equations for the viscous stress tensor $\tau$, the heat flux vector $\bar{q}$, and the mass flux vector $\bar{J}_{i}$ are required. In the absence of detailed knowledge on the true rheology of the fluid, Newtonian behavior is often assumed. Thus, for $\tau$ the following expression is used:

$$
\tau=-\left\{\left(\lambda-\frac{2}{3} \mu\right)(\nabla \cdot \bar{u}) \mathrm{I}+\mu\left((\nabla \bar{u})+(\nabla \bar{u})^{T}\right)\right\},
$$


where $\lambda$ and $\mu$, respectively, represent the bulk viscosity and the shear viscosity. In dense gases and liquids the bulk viscosity $\lambda$ can probably be neglected (Bird $e t$ al., 1960). For the heat flux vector $\bar{q}$ and the mass flux vector $\bar{J}_{i}$, respectively, Fourier's law and Fick's law are often used:

$$
\begin{gathered}
\bar{q}=-k \nabla T, \\
\overline{J_{i}}=-D_{i} \rho \nabla \omega_{i} .
\end{gathered}
$$

where $k$ represents the thermal conductivity. $T$ the temperature. and $D_{i}$ the Fickian diffusion coefficient of species $i$. Equation ( 9 ) for the description of the conductive heat flux is only valid for isotropic media: for anisotropic media the heat flux vector $\bar{q}$ should be written as the dot product of the conductivity tensor and the temperature gradient. Fick's law for the description of diffusional transport is. strictly speaking, only valid for the description of systems where (very) low concentrations of the relevant species prevail. For more general expressions the interested reader is referred to Bird et al. (1960).

The conservation equations. Eqs. (4)-(7) are also valid for the description of turbulent flows, but within the context of CFD a very high resolution in space and time would be required to capture all the details of the turbulent flow field. Although some exciting results have been obtained in recent years (see Section IV.A.2) in the so-called direct numerical simulation (DNS) in most industrial applications, which involve turbulent flows at (very) high Reynolds numbers, this approach cannot be followed. For the description of such turbulent flows. with their rapid temporal and spatial changes of pressure and velocity. usually the concept of Reynolds decomposition (Tennekes and Lumley, 1977: Warsi. 1993) is invoked where the instantilneous value of each variable $X$ appearing in the balance equations is represented as the sum of its time-averaged $X$ and a fluctuating component $X^{\prime}$ :

$$
X=\bar{X}+X^{\prime \prime} .
$$

Substitution of the expressions for the instantaneous $X$ values in the conservation equations, Eq. (4)-(7), followed by some kind of suitable time-averaging procedure leads to the time-averaged conservation equations for turbulent flow. These equations in fact show a very close resemblance to the original equations: The instantaneous pressure and velocity are replaced by the corresponding timeaveraged quantities, whereas the viscous stress tensor $\tau$ is replaced by the sum of $\tau$ (now appearing with time-averaged velocity) and the so-called Reynolds stress tensor $\tau^{(n)}$. The physical origin of the Reynolds stresses can be related to the interaction of the fluctuating fluid motion with the time-averaged fluid motion. Similarly, the heat flux and mass flux appearing in the time-averaged conservation equations for turbulent flow [corresponding. respectively, to Eqs. (6) and (7)] consist of the sum of the molecular flux expressions for $\bar{q}$ and $\bar{J}_{i}$ (now appearing 
with, respectively, time-averaged temperature and mass fraction of species $i$ ) and the turbulent or eddy contributions. For the treatment of the source terms appearing in Eq. (7), which very often depend in a nonlinear manner on the mass fractions of the species involved in the chemical transformation, special strategies are required depending on ratio of the characteristic time for chemical transformation and the characteristic time for mixing (Pope, 1994; Fox, 1996). For nonisothermal chemically reactive systems, the proper averaging of the heat source term $S_{\mathrm{l}}$, which depends on the temperature via an Arrhenius type expression (Levenspiel. 1962), should also be taken into account. The principal difficulty in modeling turbulent flow lies in the specification of the Reynolds stresses (and turbulent energy and species $i$ mass flux), which should be specified in terms of the time-averaged variables. This so-called closure problem constitutes a formidable problem for the fluid dynamicist and considerable effort has been made to develop the corresponding closure laws. For the description of turbulent flows a semiempirical turbulence model is often invoked to calculate the Reynolds stresses. A turbulence model is defined as a set of equations (algebraic or differential) that determines the turbulent (momentum) transport terms in the flow equations governing the time-averaged or mean flow. It should be stressed that the turbulence model does not simulate the details of the fluctuating fluid motion, merely its effect on the time-averaged fluid motion. The nature and the degree of refinement contained in the turbulence model will determine the range of flows for which its has true predictive power. Among the turbulence models most often applied one can mention (Rodi, 1980):

- Constant eddy viscosity model

- Prandtl's mixing length model

- Prandtl-Kolmogorov model

- $k-\epsilon$ model

- Algebraic stress model (ASM)

- Reynolds stress model (RSM)

All of these models require some form of empirical input information, which implies that they are not general applicable to any type of turbulent flow problem. However, in general it can be stated that the most complex models such as the ASM and RSM models offer the greatest predictive power. Many of the older turbulence models are based on Boussinesq's (1877) eddy-viscosity concept, which assumes that, in analogy with the viscous stresses in laminar flows, the Reynolds stresses are proportional to the gradients of the time-averaged velocity components:

$$
\tau_{i j}^{(l)}=-\mu^{(l)}\left[\frac{\partial U_{i}}{\partial x_{j}}+\frac{\partial U_{j}}{\partial x_{i}}\right]+\delta_{i j} \frac{2}{3} \rho k,
$$


where $\mu^{(t)}$ represents the turbulent viscosity, which, in contrast with the molecular viscosity $\mu$, is not a fluid property but a property of the turbulent flow. In Eq. (12) $U_{i}$ and $U_{j}$ represent time-averaged velocity components. Within the framework of the models that use the eddy-viscosity concept, the task of the turbulence model is the description, by means of algebraic or differential equations, of the turbulent viscosity $\mu^{(t)}$.

The constant eddy-viscosity model and Prandtl's mixing length model belong to the class of zero-equation models since no transport equations are involved for the turbulence quantities. In fact, the constant eddy-viscosity model cannot be regarded as a true turbulence model since the appropriate value of $\mu^{(t)}$ is usually fitted from experimental data. Experience has shown that mixing length models are not suitable when convective or diffusive transport processes of turbulence are important. The Prandtl-Kolmogorov model belongs to the class of singleequation models because one conservation equation for the turbulent kinetic energy $k$ is solved. It overcomes the aforementioned problem of the mixing length model because a transport equation for the characteristic velocity scale of turbulence is solved for. The weak point in this model is the specification of the characteristic length scale $L$, which is required in the Kolmogorov-Prandt expression for the turbulent viscosity:

$$
\mu^{(t)}=C_{\mu} \rho \sqrt{k} L
$$

where $C_{\mu}$ represents an empirical constant. Especially for complex flows it is difficult to specify the length scale (distribution) and therefore two-equation models such as the $k-\epsilon$ model have become more popular because here an additional transport equation is invoked to obtain the length scale (distribution) $L$. In the $k-\epsilon$ model two transport equations are solved for the turbulent kinetic energy $k$ and the viscous dissipation rate $\epsilon$. The expression for the turbulent viscosity is given by:

$$
\mu^{(i)}=C_{\mu} \rho \frac{k^{2}}{\epsilon}
$$

Computational experience has revealed that the two-equation models, employing transport equations for the velocity and length scales of the fluctuating motion. often offer the best compromise between width of application and computational economy. There are, however, certain types of flows where the $k-\epsilon$ model fails, such as complex swirling flows, and in such situations more advanced turbulence models (ASM or RSM) are required that do not involve the eddy-viscosity concept (Launder, 1991). According to the ASM and the RSM the six components of the Reynolds stress tensor are obtained from a complete set of algebraic equations and a complete set of transport equations. These models are conceptually superior with respect to the older turbulence models such as the $k-\epsilon$ model but computationally they are also (much) more involved. 
Due to the advances in computer technology and numerical solution procedures two powerful simulation types of turbulent flows have recently received particular attention, namely, direct numerical simulation (DNS) and large eddy simulation (LES). As stated earlier turbulent flows are also governed by the Navier-Stokes equations and in principle the solution of these equations with a sufficiently high temporal and spatial resolution should provide all the details of the turbulent flow without the necessity of turbulence modeling. Due to the fact that a turbulent motion contains elements with a linear dimension which is typically $O\left(10^{-3}\right)$ smaller than the linear dimension of the macroscopic flow domain, a DNS simulation in three dimensions would require roughly $O\left(10^{9}\right)$ grid points, which is still far beyond the current storage capacity of present-day computers. The estimate given here is only a very rough one and corresponds to relatively high Reynolds numbers. It should be mentioned here that the required number of grid points strongly depends on the nature of the turbulent flow (homogeneous isotropic turbulent flow or homogeneous shear flow) and also on the Reynolds number since the ratio of the dimension of the macroscopic system and the dimension of the smallest eddies present in the turbulent flow depends on the Reynolds number: The smaller the Reynolds number the smaller this ratio. For turbulent channel flow the ratio of the channel width $2 \delta$ to the scale of the smallest eddies $\lambda$ is proportional to $\left(\operatorname{Re}_{\delta}\right)^{0.9}$ where the Reynolds number $\operatorname{Re}_{\hat{\delta}}$ is defined as:

$$
\operatorname{Re}_{\delta}=\frac{U_{\mathrm{c}} \delta}{\nu},
$$

where $U_{\mathrm{c}}$ is the time-averaged velocity in the centre of the channel. On basis of the DNS performed by Kim et al. (1987) at $\mathrm{Re}_{\delta}=3300$ using $2 \cdot 10^{6}$ grid points, Reynolds (1991) has estimated the number of grid points $N_{x y}$ required for a DNS computation with comparable resolution to that of Kim et al. (1987) as:

$$
N_{x y z}=2 \cdot 10^{6}\left[\frac{\mathrm{Re}_{\delta}}{3300}\right]^{2.7}
$$

In a LES the spatial resolution of the computational mesh is (deliberately) chosen in such a manner that only the large-scale turbulent motion (eddies) is resolved. The consequence of this approach is the need to use subgrid models, which in fact model the turbulent stresses on a scale smaller than the computational grid. Due to the fact that the small-scale turbulence is isotropic, the specification of subgrid models is (far) less difficult in comparison with the aforementioned closure models for the Reynolds stresses. The advantage of LES in comparison with DNS is its possibility to study (with a given number of grid points or control volumes) turbulent flows at (significant) higher Reynolds numbers. 


\section{Multiphase Systems}

For each continuous phase $k$ present in a multiphase system consisting of $N$ phases, in principle the set of conservation equations formulated in the previous section can be applied. If one or more of the $N$ phases consists of solid particles, the Newtonian conservation laws for linear and angular momentum should be used instead. The resulting formulation of a multiphase system will be termed the local instant formulation. Through the specification of the proper initial and boundary conditions and appropriate constitutive laws for the viscous stress tensor, the hydrodynamics of a multiphase system can in principle be obtained from the solution of the governing equations.

However. for most systems of practical interest, the analysis of multiphase systems on basis of the local instant formulation is intractable, even for existing and near-future supercomputers, and consequently some kind of simplification must be made. From a computational point of view this state of the art bears some resemblance to the problems encountered in DNS of turbulent flows.

The aforementioned simplification can be achieved through a continuum mathematical description of the multiphase system. There is extensive literature on the derivation of continuum equations for multiphase systems; the interested reader is referred to Ishii (1975). The derivation of the continuum equations is usually based on spatial averaging techniques where the point hydrodynamic variables are replaced by local averaged variables. The resulting multifluid formulation can be solved by appropriate numerical methods, which in fact generalize the well-known single-phase solution procedure of Patankar (1980). For multiphase isothermal systems involving laminar flow the conservation equations for mass and momentum are respectively given by:

$$
\begin{gathered}
\frac{\partial}{\partial t} \rho_{k}+\left(\nabla \cdot \rho_{k} \bar{u}_{k}\right)=R_{k} . \\
\frac{\partial}{\partial t}\left(\rho_{k} \bar{u}_{k}\right)+\left(\nabla \cdot \rho_{k} \bar{u}_{k} \bar{u}_{k}\right)=-\epsilon_{k} \nabla p-\left(\nabla \cdot \epsilon_{k} \tau_{k}\right)+\sum_{i=1}^{N} \bar{M}_{k l}+\bar{S}_{k}+\rho_{k} \bar{g}
\end{gathered}
$$

where $\rho_{k}, \bar{u}_{k}, \epsilon_{k}$, and $\tau_{k}$ represent, respectively, the macroscopic density, velocity, volume fraction, and viscous stress tensor of the $k^{\text {th }}$ phase, $p$ the pressure, $R_{k}$ a source term describing mass exchange between phase $k$ and the other $N-1$ phases, $\bar{M}_{k l}$ the interphase momentum exchange term between phase $k$ and phase $l$. and $\bar{S}_{k}$ a momentum source term of phase $k$ due to phase changes and external forces other than gravity. For nonisothermal multiphase systems the transport equations (17) and (18) have to be supplemented with $N$ thermal energy equations (one for each phase), whereas for multiphase systems involving chemical conversion of $M$ species, in the most general case $N M$ species conservation equations 
have to be added. The thermal energy equation and the conservation equation for species $i$ present in phase $k$ are respectively given by:

$$
\begin{gathered}
\frac{\partial}{\partial t}\left(\rho_{k} e_{k}\right)+\left(\nabla \cdot \rho_{k} e_{k} \bar{u}_{k}\right)=-p\left[\frac{\partial \epsilon_{k}}{\partial t}+\left(\nabla \cdot \epsilon_{k} \bar{u}_{k}\right]-\left(\nabla \cdot \bar{q}_{k}\right)\right. \\
+\sum_{i=1}^{N} E_{k l}+S_{h, k} \\
\frac{\partial}{\partial t}\left(\rho_{k} \omega_{k, i}\right)+\left(\nabla \cdot \rho_{k} \omega_{k, i} \bar{l}_{k}\right)=-\left(\nabla \cdot \bar{J}_{k, i}\right)+S_{k, i}
\end{gathered}
$$

where $e_{k}$ represents the internal energy per unit mass of phase $k, \vec{q}_{k}$ the heat flux vector due to molecular transport in phase $k, E_{k l}$ the interphase energy transfer term between phase $k$ and phase $l, S_{h}, k$ the volumetric heat production rate in phase $k, \omega_{k, i}$ the mass fraction of species $i$ in phase $k, J_{k, i}$ the mass flux vector of species $i$ in phase $k$ due to molecular transport, and $S_{k, i}$ the net production rate of species $i$ in phase $k$ per unit volume. For closure of the conservation equations expressions similar to Eqs. (9) and (10) are used but it should be kept in mind that the transport coefficients (conductivities and diffusivities) now represent elfective transport coefficients that depend, amongst others, on the volume fractions of the $N$ phases. It should be mentioned here that in many situations the $M$ species are not present in all phases and in such circumstances (far) fewer conservation equations have to be formulated (and solved). Analogous to the situation for single-phase flows, the conservation equations (17)-(20) are also valid for the description of turbulent multiphase flows, but problems similar to those encountered in single-phase turbulent flows have to be circumvented. In this connection the strategy parallels the development presented in the previous section. However, due to the complexity of multiphase flows, the uncertainties introduced through the modeling of, for instance, the Reynolds stresses significantly increase. Additional difficulties arise due to the fact that closure equations for interphase transport of mass, momentum, and heat have also to be specified.

In multiphase systems involving one or more dispersed phases an alternative to the aforementioned complete continuum representation is possible by adopting a Lagrangian description for these phases. The advantages of this mixed Eulerian-Lagrangian approach are its greater generality and flexibility with respect to the incorporation of microscopic transport phenomena, whereas its relatively high (compared to completely Eulerian approaches) computational load constitutes its most important disadvantage. However, also from a computational point of view a mixed Eulerian-Lagrangian approach can offer certain advantages (see Section IV,B, 1). If a Lagrangian description is adopted to represent the dispersed phase, for each individual particle (or bubble or droplet) an equation of motion is solved:

$$
m_{i} \frac{d}{d t} \bar{v}_{i}=\sum \bar{F}_{i}
$$


where $m_{i}, \bar{v}_{i}$ represent, respectively, the mass and velocity of the $i$ th particle and $\Sigma \bar{F}_{i}$ the sum of the forces acting on the $i$ th particle. Forces due to gravity, drag, virtual mass, vorticity in the continuous phases, and electrical forces can be included in this term. The particle position vector is calculated from:

$$
\frac{d}{d t} \bar{x}_{i}=\bar{v}_{i}
$$

The solution of differential equations (21) and (22) can be obtained with standard numerical integration techniques.

Depending on the volume fraction of the dispersed phase, one-way coupling or two-way coupling between the dispersed phase and the continuous phase prevails. In systems involving (turbulent) multiphase flow at very small volume fraction of the dispersed phase, say, smaller than $10^{-6}$, one-way coupling may be assumed. At such low volume fractions the effect of the particles on the turbulence structure in the continuous phase is negligible while particle-particle interactions (i.e., collisions) do not play a role. For systems with higher volume fractions $\left(10^{-6}\right.$ to $\left.10^{-3}\right)$ the turbulence structure of the continuous phase is influenced by the dispersed phase while particle-particle interaction can still be neglected and two-way coupling between the phases has to be accounted for. With respect to the effect of the dispersed phase on the turbulence structure it can be mentioned that the ratio of the particle response time $\tau_{\mathrm{p}}$ and the Kolmogorov time scale $\tau_{\mathrm{K}}$ determines whether the particles will enhance the production rate of turbulence energy $\left(\tau_{\mathrm{p}} / \tau_{\mathrm{K}}>100\right)$ or increase the dissipation rate of turbulence energy $\left(\tau_{\mathrm{p}} / \tau_{\mathrm{K}}<100\right)$. For still higher volume fractions of the dispersed phase particle-particle interaction (i.e., collisions) becomes important and four-way coupling has to be accounted for (see Fig. 3; from Elgobashi, 1991). In this case an integrated modeling approach is required combining features of molecular dynamics (MD) to deal effectively with the huge number of particle-particle and/or particle-wall collisions, and CFD to obtain the velocity distribution in the continuous phase (see Section VI).

\section{B. NumERICAL TECHNIQUES}

We will not attempt here to give a detailed explanation of the numerical aspects (fundamentals of discretization, error estimates, and error control) of CFD since a number of excellent texts are available in the literature that deal in depth with this matter (Fletcher, 1988a,b; Hirsch, 1988, 1990). First some general aspects of the numerical techniques used for solving fluid flow problems are discussed and, subsequently, a distinction is made between single-phase flows and 


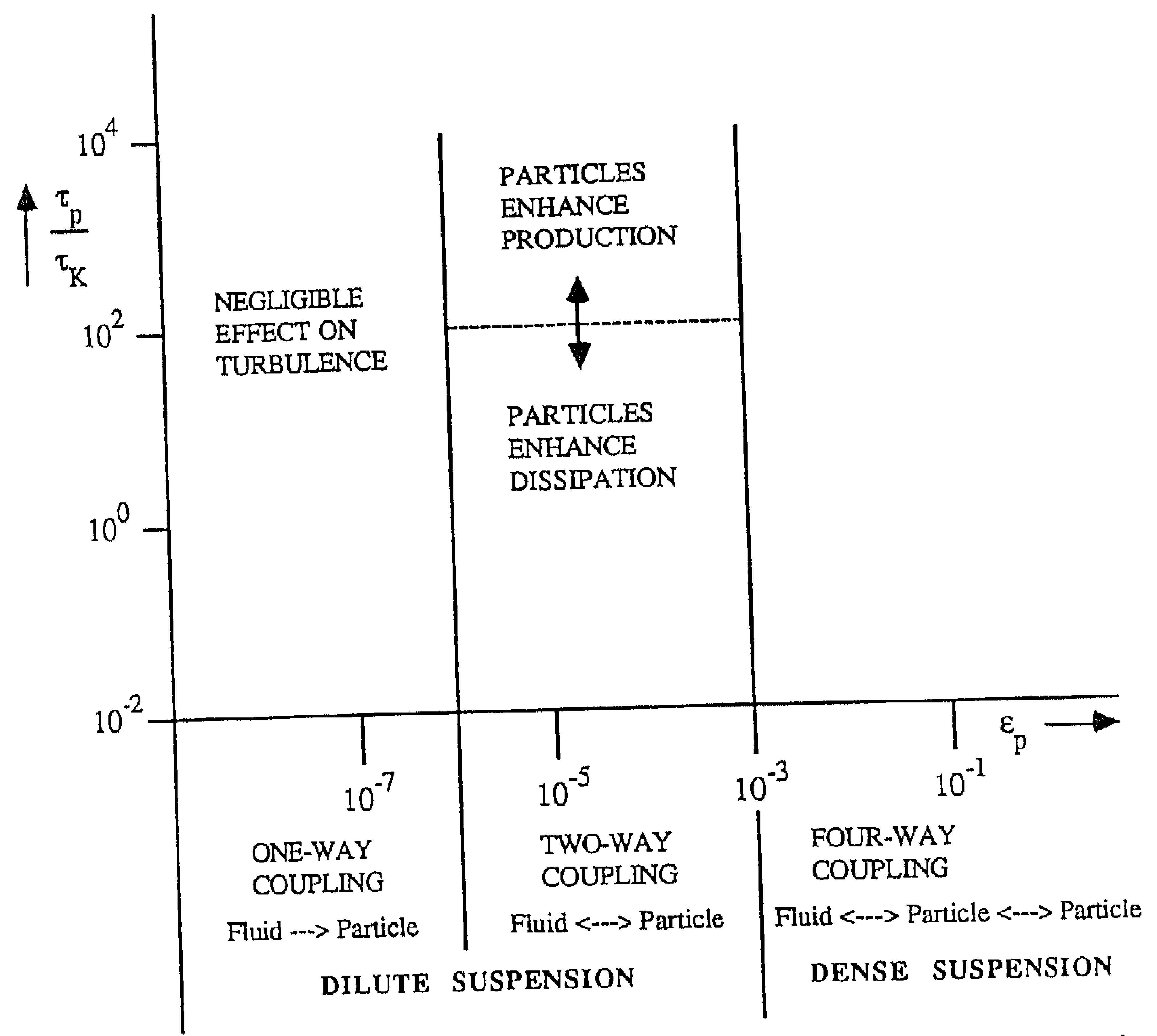

FIG. 3. Map of how regimes in particle-laden flows. (From Levenspiel, O., "Chemical Reaction Engineering." John Wiley \& Sons, New York, 1962. Reprinted by permission of John Wiley \& Sons. Inc.)

multiphase flows. In general the solution of a fluid flow problem involves the following steps:

1. grid generation with the aid of:

- Algebraic methods (Wang and Hoffman, 1986; Marcum and Hoffman, 1988; Hoffman, 1992)

- Conformal mapping (Thompson et al., 1982, 1985; Thompson, 1982)

- Systems of (elliptic) partial differential equations (Thompson et cll., 1982, 1985; Thompson, 1982)

Numerical grid generation can be seen as a highly specialized branch of CFD. The grids generated by either of the above-mentioned methods can be divided into structured grids and unstructured grids (see Fig. 4). In a structured grid the connectivity is constant throughout the interior computational domain, whereas unstructured grids do not necessarily have a constant connectivity. In general, un- 

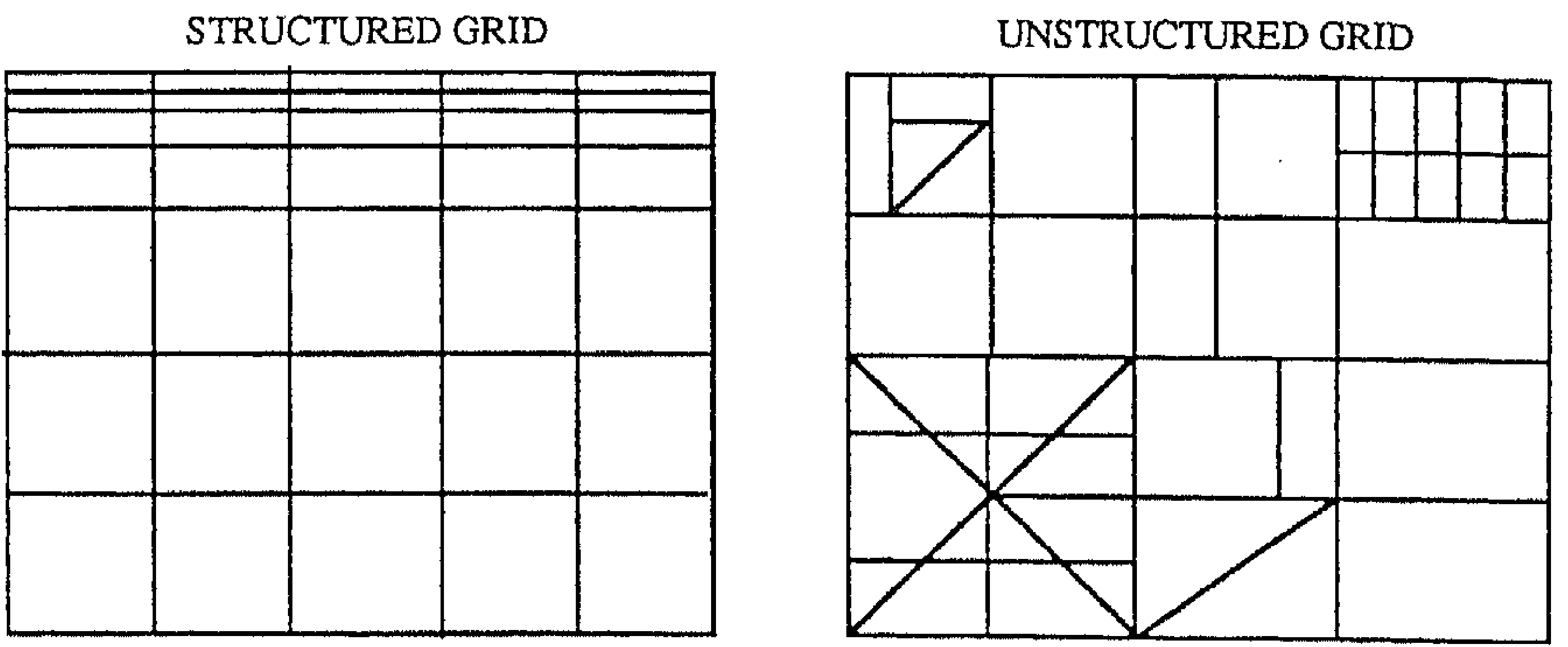

FIG. 4. Structured and unstructured grids.

structured grids offer better possibilities to represent systems with complex geometric features. Furthermore, staggered and nonstaggered (collocated) computational grids can be distinguished (see Fig. 5).

2. Discrete representation of the transport equations using:

- Finite difference methods (FDM) (Roache, 1972)

- Finite volume methods (FVM) (Patankar, 1980)

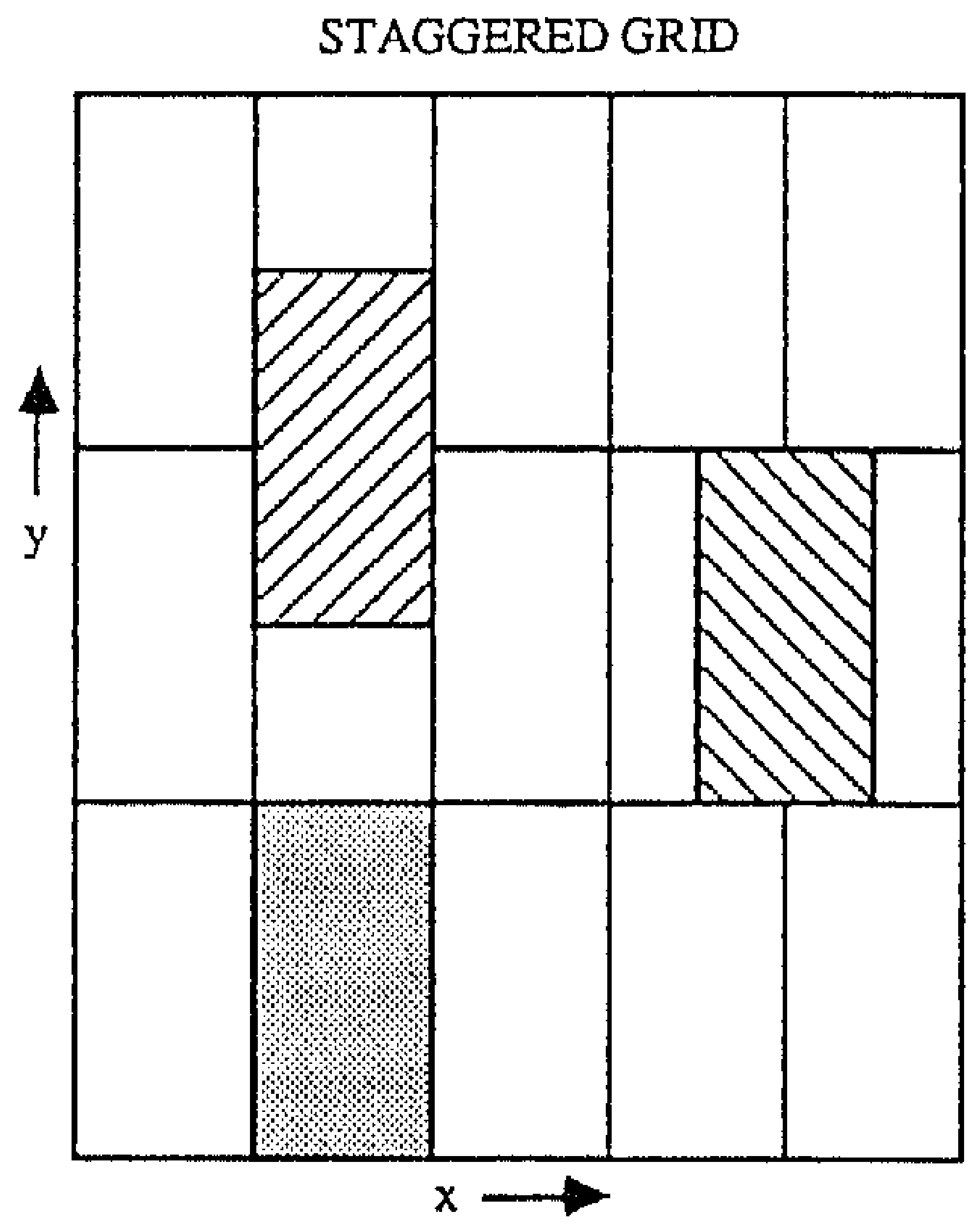

Control volume for mass

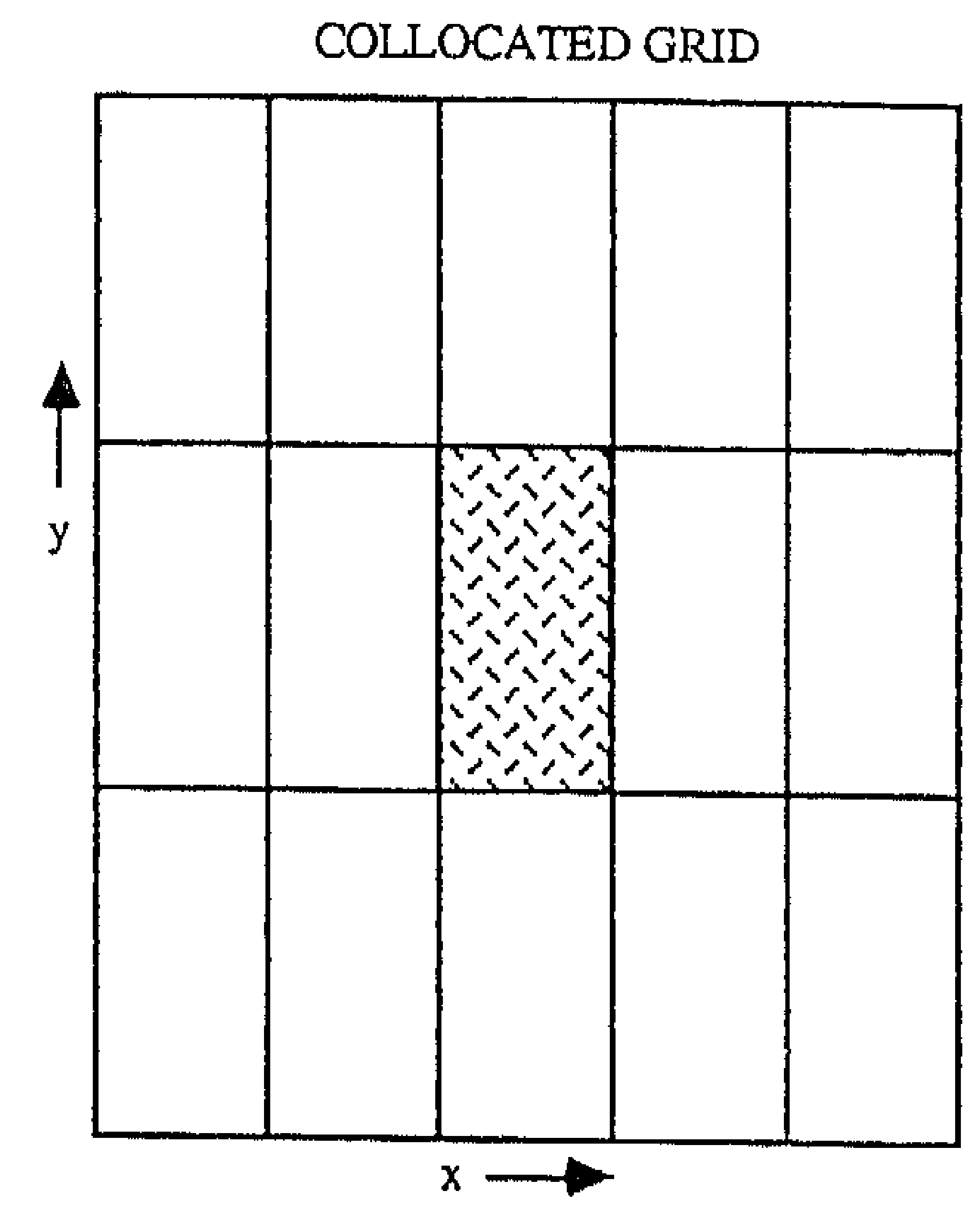

ETi? Control volume for all quantities

$\triangle \backslash \backslash \nabla$ Control volume $\mathrm{x}$-momentum

EZZZ Control volume y-momentum

FIG. 5. Staggered and collocated grids. 
- Finite element methods (FEM) (Baker, 1985; Zienkiewicz and Taylor, 1989a,b).

- Boundary element methods (BEM) (Becker, 1992)

The solution of the resulting nonlinear equations is usually achieved via an iterative algorithm. Once a converged solution has been obtained it is essential to assess the invariance of the computational results with respect to the temporal and/or spatial discretisation. This aspect is unfortunately often not addressed in computational studies due to, amongst others, computer time constraints.

3. Postprocessing:

- Contour and vector plots

- Data reduction

- Animations

Postprocessing constitutes a very important final step, especially for the chemical engineer because in many chemical processes besides fluid phenomena many other aspects have to be considered (i.e., fouling, catalyst deactivation).

\section{Single-Phase Systems}

It should be mentioned here that the discrete representation of the transport equations can be based on the so-called primary variables such as pressure and velocity components but also on the stream function vorticity formulation (Roache, 1972). The SIMPLE algorithm (Patankar, 1980) is probably the most widely employed algorithm for the solution of fluid flow problems and forms the basis of many commercial FVM-based codes (PHOENICS, FLUENT, and FLOW3D). The SIMPLE algorithm is based on a sequential solution method of the nonlinear equations, which is advantageous from a modeling point of view since additional transport equations can be added with relative ease. This solution method can cause problems in case a (very) strong coupling between chemistry and heat transfer processes prevails. Finite element-based CFD codes are mostly based on the method of weighted residuals (Baker, 1985; Zienkiewicz and Taylor, 1989a,b) and use a simultaneous solution procedure for the transport equations where typically the multidimensional Newton-Raphson method is used.

A totally different approach to solving the Navier-Stokes equations is made in alternating direction implicit (ADI) methods (Briley and McDonald, 1975) and approximate factorization implicit (AFI) methods (Beam and Warming, 1977, 1978). These methods apply an approximate spatial factorization technique to avoid the inversion of huge banded matrices and are computationally very efficient.

Another class of powerful numerical techniques that should be mentioned here are spectral and pseudospectral methods, which are often used in DNS because of 
their superior accuracy (relative to finite difference methods) in case the same resolution is used. At the same time these techniques are competitive in computational efficiency. For a detailed description of many spectral algorithms and exhaustive discussion of the theoretical aspects of these numerical methods, the interested reader is referred to Canuto et al. (1988).

An important class of fluid flow problems involves free surface flows where only the flow phenomena in a single phase are of importance despite the fact that two or more phases are present in the system. Such situations are encountered, for example, in gas-liquid two-phase flows where, due to the large density difference. from a hydrodynamic point of view the flow can be treated as a liquid flow in a vacuum. The analysis of such free surface flows is rather complex due to the fact that in addition to the fluid flow problem the position of the interface and enforcement of appropriate boundary conditions have to be dealt with.

A number of computational techniques have been proposed in the past such as the marker and cell (MAC) method and the simplified marker and cell (SMAC) method developed, respectively, by Welch et al. (1965) and Amsden and Harlow (1970). In these methods a fixed computational mesh is employed through which the fluid moves: in addition a large number of massless marker particles are used to track the interface dynamically (see Fig. 6). The movement of these marker particles is performed on basis of the local fluid flow field where area (2D) or volume (3D) weighting is applied with respect to the velocity components, which are available only at the Eulerian nodes of the computational mesh. Especially for 3D simulations the storage requirements for the marker particle based methods can become problematic and therefore more efficient (from the point of view of storage requirements) free surface computational algorithms have been devised. An interesting development in this connection is the (single-material) volume of fluid

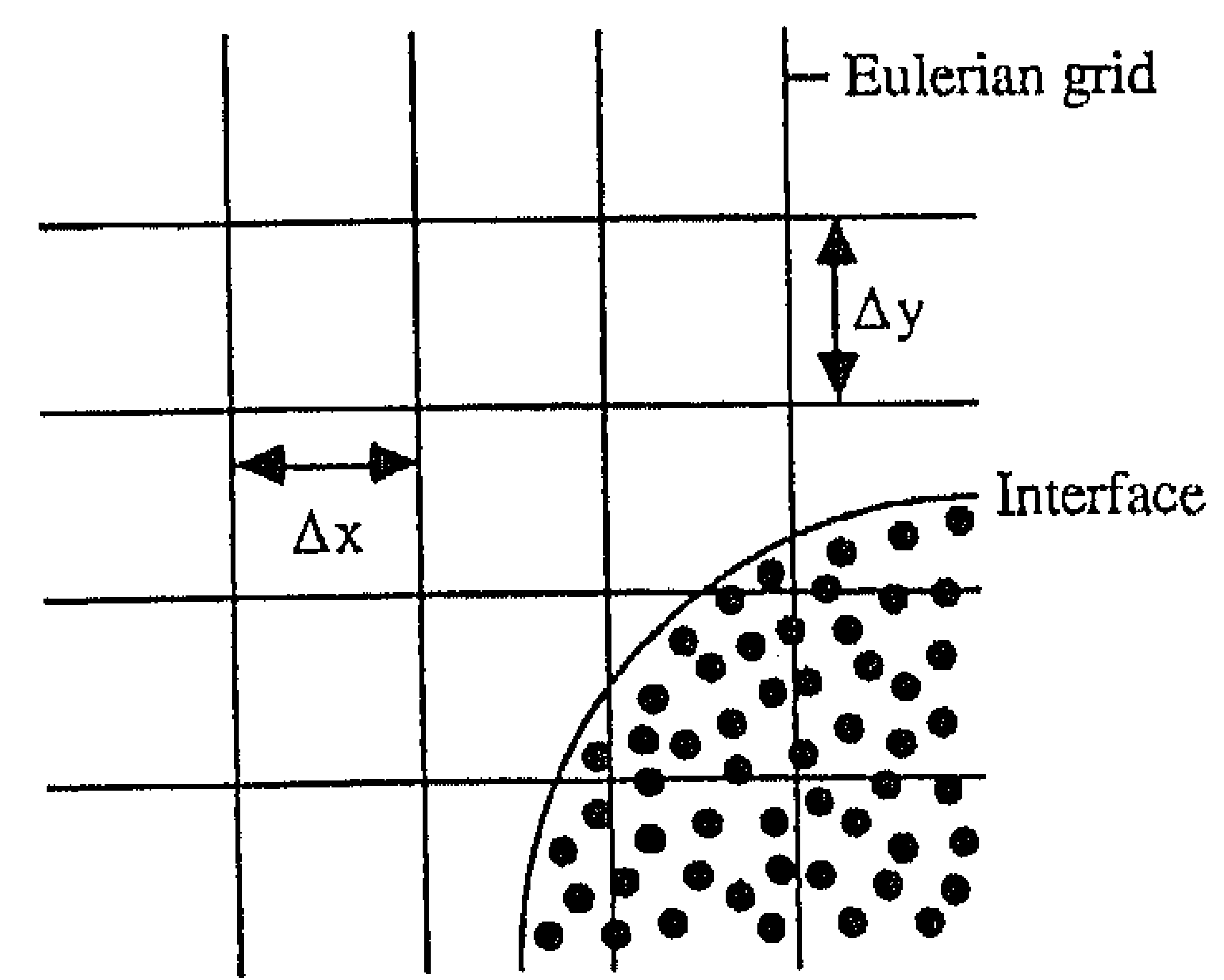

FIG. 6. Marker particles in an Eulerian grid. The marker particles are used to track the interface dynamically. 
(VOF) method (Hirt and Nichols, 1981; Nichols et al., 1980) where in addition to the Navier-Stokes equations a scalar transport equation (the so-called $F$ equation) for the fractional amount of fluid $F$ is solved for:

$$
\frac{\partial F}{\partial t}+(\nabla \cdot \bar{u} F)=0
$$

where $\bar{u}$ represents the local fluid velocity. The solution of the $F$ equation yields the $F$ values at all computational cells of the domain, and with the aid of an interface reconstruction algorithm the position and orientation of the interface can be obtained. With the VOF method it is possible to incorporate, for example. surface tension via the condition for the normal stress at the interface (Hirt and Nichols, 1981) or via the continuum surface force (CSF) model (Kothe et al., 1991).

Recently Rudman (1997) has compared the performance of various volumetracking methods for interfacial flow calculations (including the VOF method of Hirt and Nichols, 1981) and concluded that the method originally proposed by Youngs (1982) provides the best results (i.e., conservation of a sharp and welldefined interface) on various "artificial" problems including solid body rotation and shearing flow. In addition this method yielded the best results for the numerical simulation of Rayleigh-Taylor instability. Nakayama and Mori (1996) combined a marker particle approach and a finite element method (FEM) based solution methodology to model time-dependent free surface flows in complex geometries. Udaykumar et al. (1996) presented a mixed Eulerian-Lagrangian method for fiuid flows with complex and moving boundaries. They also used marker particles to represent and track the interface in the Eulerian grid. A special feature of their computational approach is the possibility to embed solidification or melting processes, with the associated dynamic evolution of the phase boundaries, in the flow simulation.

\section{Multiphase Systems}

For multiphase systems a rough distinction can be made between systems with separated flows and those with dispersed flows. This classification is not only important from a physical point of view but also from a computational perspective since for each class different computational approaches are required. For multiphase systems involving multiphase flow both Eulerian, mixed EulerianLagrangian, and two-material free surface methods can be used. An excellent review on models and numerical methods for multiphase flow has been presented by Stewart and Wendroff (1984). A similar review with emphasis on dilute gasparticle flows has been presented by Crowe (1982).

a. Eulerian Methods. The development of Eulerian computational methods for multiphase flows was pioneered by Harlow and Amsden $(1974,1975)$ at the Los 
Alamos scientific laboratory. Their implicit continuous Eulerian (ICE) method formed the basis for many (Rivard and Torrey, 1977, 1979; Cook et al., 1981) later developments.

b. Eulerian-Lagrangian Methods. For dispersed multiphase flow roughly speaking three different situations and corresponding computational strategies can be distinguished (also see Fig. 7):

1. Dilute flows where on the average less than one particle is present in a computational cel]

2. Dense flows where a relatively high number of particles are present in a computational cell

3. Dilute or dense flows where a large number of computational cells is contained in a single particle

We should mention here that the dispersed phase could also consists of drops or bubbles, which could. in principle, be deformable.

The situation for case 1 arises when suspensions are relatively dilute and the particles are small: Depending on the exact value of the volume fraction of the dispersed phase, one-way coupling or two-way coupling prevails (Pan and Banerjee, 1996a). With one-way coupling particles are being moved in response to the fluid motion without feeding back effects to the continuous phase, whereas in two-way coupling feedback effects are taken into account. As discussed in detail by Pan and Banerjee (1996a) care must be taken to implement two-way coupling correctly. Examples of one-way coupled calculations are those of Squires and Eaton (1990) and Pedionotti et al. $(1992,1993)$ who both used DNS to study, respectively, particle segregation in homogeneous isotropic turbulent flow and particle segregation in a turbulent channel flow. For two-way coupling, different computational strategies are used depending on whether there is only one particle in a computational cell or many.

In case 2 we deal with dense flows and in our computational strategy four-way coupling has to be accounted for since there is not only mutual interaction between the suspended particles and the continuous phase but also particle-particle interactions (i.e., collisions).

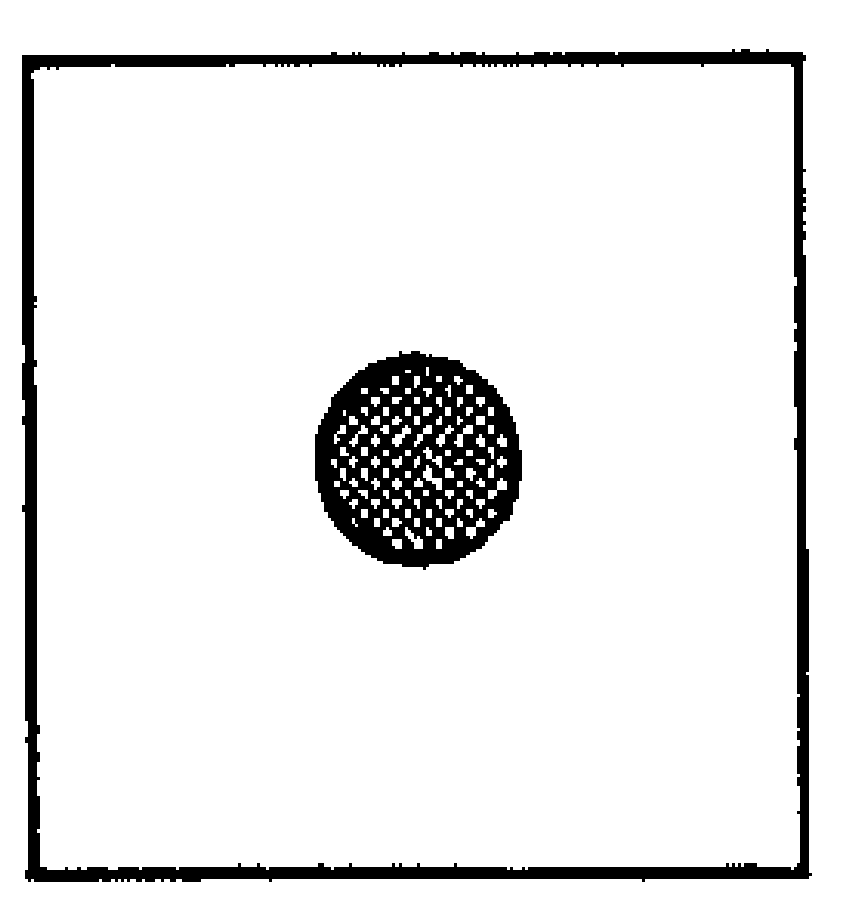

case a

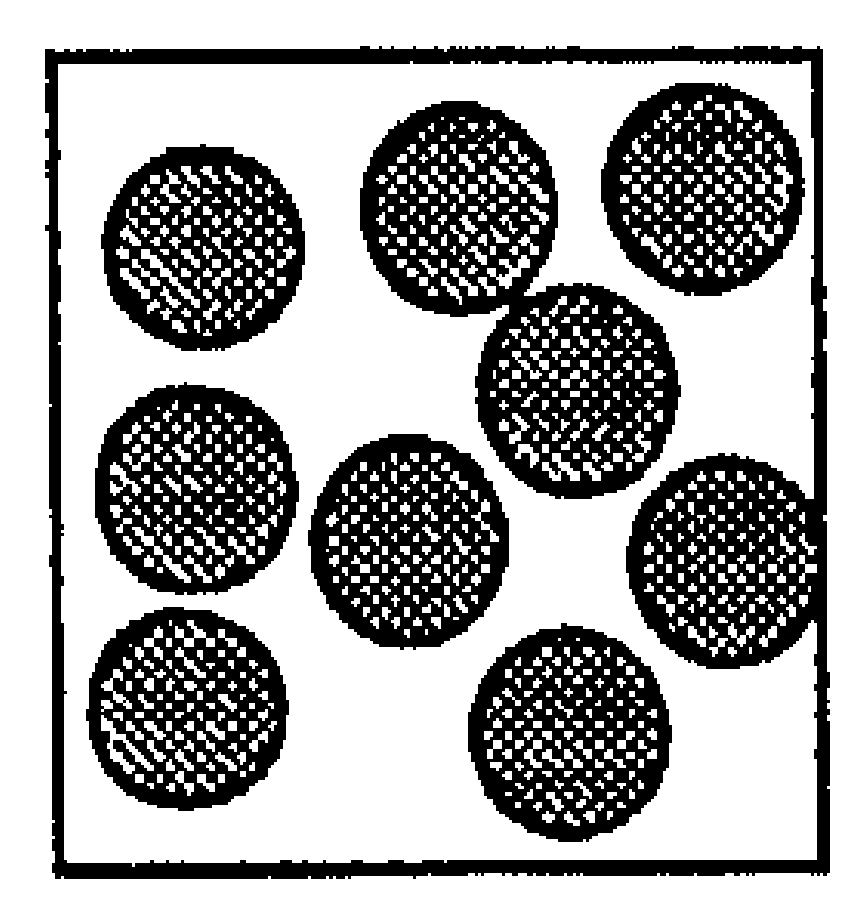

case b

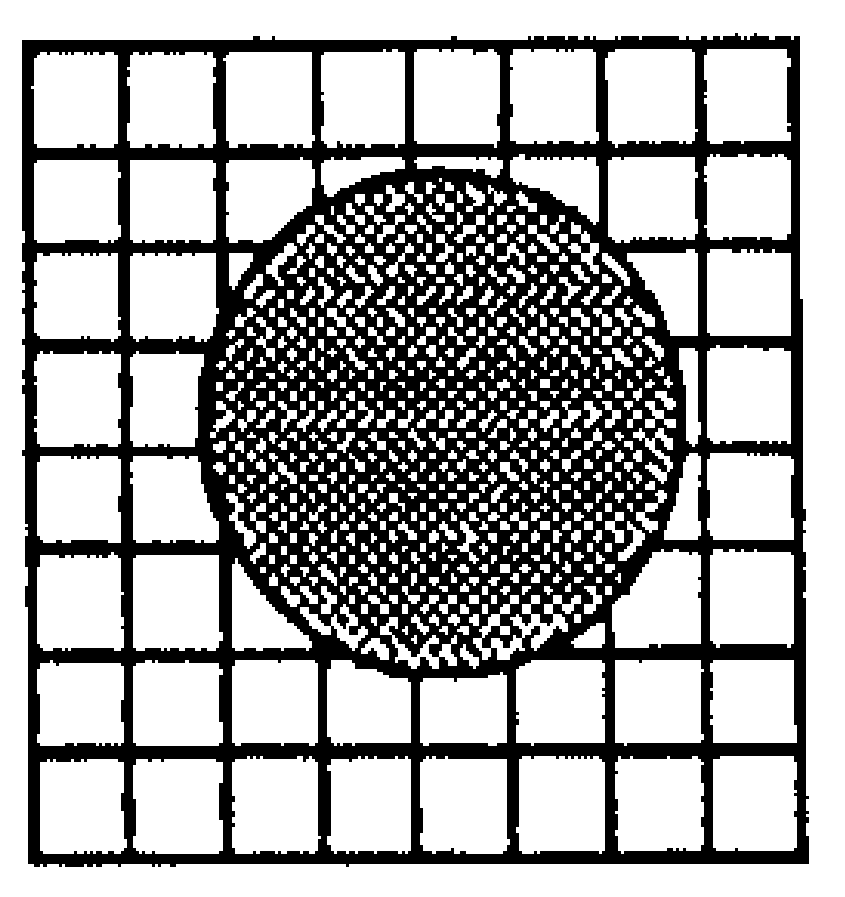

case c

FIG. 7. Different situations that can be distinguished in modeling dispersed multiphase flow. 
In case 3 the relative size of the particles (with respect to the computational cells) is large enough that they contain many hundreds or even thousands of computational cells. It should be noted that the geometry of the particles is not exactly represented by the computational mesh and special, approximate techniques (i.e. body force methods) have to be used to satisfy the appropriate boundary conditions for the continuous phase at the particle surface (see Pan and Banerjee, 1996b). Despite this approximate method, the empirically known dependence of the drag coefficient versus Reynolds number for an isolated sphere could be correctly reproduced using the body force method. Although these computations are at present limited to a relatively low number of particles they clearly have their utility because they can provide detailed information on fluid-particle interaction phenomena (i.e., wake interactions) in turbulent Hows.

c. Two-Material Free Surface Methods. The MAC or SMAC and VOF techniques can also be applied to study two-material or two-phase free surface flows. For details the interested reader is respectively referred to Welch et al. (1965) or Amsden and Harlow (1970), Hirt and Nichols (1981), and Nichols et al. (1980).

\section{Existing Software PaCKages}

Because of the mathematical and numerical difficulties involved in the development of a CFD package this activity has been undertaken mainly by specialists where the user is kept away from the details of the solution procedures. Nowadays there exist many user-friendly CFD packages which allow the engineer to set up and solve complex fluid flow problems with relative ease. First we would like to make a few comments on the selection of commercially available CFD packages and the aspects which should be kept in mind in this connection:

1. User requirements. Does the user merely want to use a CFD package in its existing form to study fluid flow and related phenomena or does the user intend to modify and further develop the physical models incorporated in this package? For the second class of users it would be very beneficial to have access to the source code of the CFD package. In this last respect it is worrying that the claimed future trend is toward fewer and fewer CFD codes being available in source code.

2. Geometry. Do we have to deal with relative simple geometries or very complex ones? In equipment encountered in many industrial processes we have to deal with complex geometries and in such situations body fitted coordinates (BFCs) may be used to fit the contours of the volume in question. To provide for greater flexibility with respect to distribution of grid points in regions of the domain (possibly adaptive) where steep gradients are 
expected, nonstructured grids might be advantageous. Both CFD codes based on finite volume and finite element formulations are suitable for analysis of fluid flow in complex geometries.

3. Dimensions. The number of relevant dimensions for the class of fluid flow problems to be studied should be identified. Although in many cases it will be necessary to solve the full three-dimensional transport equations it is often wise to first perform preliminary two-dimensional calculations in order to gain some computational experience for the system of interest.

4. Preprocessing. Especially for analysis of fluid flow phenomena in complex geometries, the availability of efficient and easy-to-use preprocessors for both problem definition and grid generation is of crucial importance. Fortunately, most commercially available CFD packages meet this requirement.

5. Numerical method. The choice of the proper numerical solution procedure should be left to specialists. Even when a particular CFD package has been chosen, the user can usually choose different solution strategies for the linearized equations (point or line relaxation techniques versus whole field solution techniques) and associated values of the relaxation parameters. The proper choice of relaxation factors to obtain converged solutions (at all) within reasonable CPU constraints is a matter of experience where cooperation between the engineer and the specialist is required. This is especially true for new classes of fluid flow problems where previous experience is nonexistent.

6. Turbulence models. Do we have to consider turbulent flow (in complex) geometries or not? Especially in systems with complex geometries where turbulent flows prevail, simple turbulence models may fail and in such circumstances advanced turbulence models (ASM or RSM) are required.

7. Multiphase capabilities. For CFD analysis of multiphase flows using commercially available CFD packages it is very important that the CFD package provide for an open programming environment where the user can implement his or her own physical submodels.

8. Postprocessing. Usually the direct result of a simulation consists of large arrays of numbers that have to be converted somehow into more appealing information. Fortunately, most CFD packages incorporate various postprocessing facilities to convert the basic data into beautiful color plots which show the spatial distribution of key variables. Despite these postprocessing facilities it is very important that some kind of further data reduction can be achieved which enables the engineer to present the computational results in an efficient and compact manner. For multiphase systems, where the flow is often nonstationary, it is furthermore of importance that the simulation results can be visualized dynamically, that is, with computer-generated movies.

The existing software packages can be divided into two broad categories, namely, commercially available general-purpose codes such as PHOENICS, 
FLUENT, FLOW3D, ASTEC, and FIDAP, and codes that have been developed at universities and industrial laboratories for more specific applications:

1. FLUFIX, for modeling fluidized bed combusters (Chang et al., 1989), at the Argonne National Laboratory (USA)

2. KIVA I and II, for analysis of internal combustion engines (Amsden, 1985 , 1989), at the Los Alamos National Laboratory (USA)

3. MELODIF and ESTET-ASTRID, for various applications in the field of energy production, at Electricité de France (Simonin, 1996)

The development of the modeling capability of commercially available codes such as PHOENICS, FLUENT, and FLOW3D has proceeded rapidly and it is to be expected that this development will continue in the near future. It is interesting to note that some vendors of general-purpose CFD packages have recently started to offer specialized versions of their codes for certain applications: POLYFLOW for CFD analysis of polymer materials processing applications (i.e., extruders), NEKTON for CFD analysis of flow phenomena in thin-film coating processes, and RAMPANT for CFD analysis of complex (external) flows. This section concludes with the specification of some of the current modeling capabilities embedded in these codes:

1. Steady-state or transient two-dimensional (2D) and three-dimensional (3D) flows in standard geometries involving Cartesian, cylindrical, or spherical coordinates and complex geometries involving BFCs with adaptive grids

2. Laminar flows involving Newtonian and non-Newtonian fluids

3. Turbulent flows with simple closure models (eddy viscosity, mixing length. $k-\epsilon$ ) or complex closure models (ASM, RSM, RNG) for the Reynolds stresses

4. Compressible and incompressible flows

5. Flows involving mixing and/or chemical reaction

6. Flows in porous media

7. Multiphase flows using Eulerian multiffuid approaches

8. Multiphase flows involving dispersed phases (particles, droplets or bubbles) using mixed Eulerian-Lagrangian approaches both with one-way and two-way coupling

9. Flows involving radiative heat transfer

10. Complex boundary conditions

\section{Application to Chemical Reaction Engineering}

As stated in the introductory section, applications of CFD may be divided into broad categories, namely, those involving single-phase systems and those involv- 
ing multiphase systems. The motivation for this distinction is due to (1) large differences in degree of complexity of physical description and (2) large differences in numerical solution strategies.

\section{A. Single-Phase Systems}

Within single-phase systems a further distinction can be made between systems involving (1) laminar flows, (2) turbulent flows, (3) flows with complex rheology, and (4) fast chemical reactions. Of course certain systems exist that fall into several of these classes and therefore also possess their main characteristics. These types of systems are discussed in more detail.

\section{Laminar Flows}

In many practical applications the chemical engineer has to deal with flows in complex geometries of which flows in curved pipes or channels and bends and flows around bodies with various shapes (i.e., cylinders and spheres) positioned inside ducts are well-known examples. As far as laminar flow systems are concerned, accurate flow field prediction, even in systems with great geometrical complexity, can be achieved nowadays with the aid of CFD and probably the accuracy of the simulations exceeds that of experiments. For systems with great geometrical complexity a number of computational approaches are possible such as finite difference or finite volume methods that invoke curvilinear coordinates (Thompson et al., 1982) or finite element methods (Baker, 1985; Zienkiewicz and Taylor, 1989a,b). In the excellent books written by Baker (1985) and Zienkiewicz and Taylor (1989a.b) and the references cited therein numerous applications of (laminar) flows involving complex geometries (ducts with complex shape and/or immersed bodies, stirred vessels, etc.) can be found. A few illustrative examples of laminar flow around bodies present inside ducts and laminar flow in stirred vessels are highlighted.

a. Laminar Flow in Ducts. An example of the application of curvilinear coordinates can be found in the paper by Wang and Andrews (1995) who presented simulation results for laminar flow of a Newtonian liquid in a helical duct. They used a helical coordinate system to describe the geometry of the flow domain and used a modified MAC method (Welch et al., 1965) to solve the two-dimensional incompressible Navier-Stokes equations. Specific features of the flow in this geometry are a relatively high frictional flow resistance and the occurrence of a socalled secondary flow pattern. In Fig. 8a the helical coordinate system is shown, whereas in Fig. $8 \mathrm{~b}$ the effect of the pressure gradient in the circumferential direction $(\partial \phi / \partial \theta)$ on the structure of the secondary flow is shown in terms of the streamline pattern. Note that with increasing circumferential pressure gradient the 

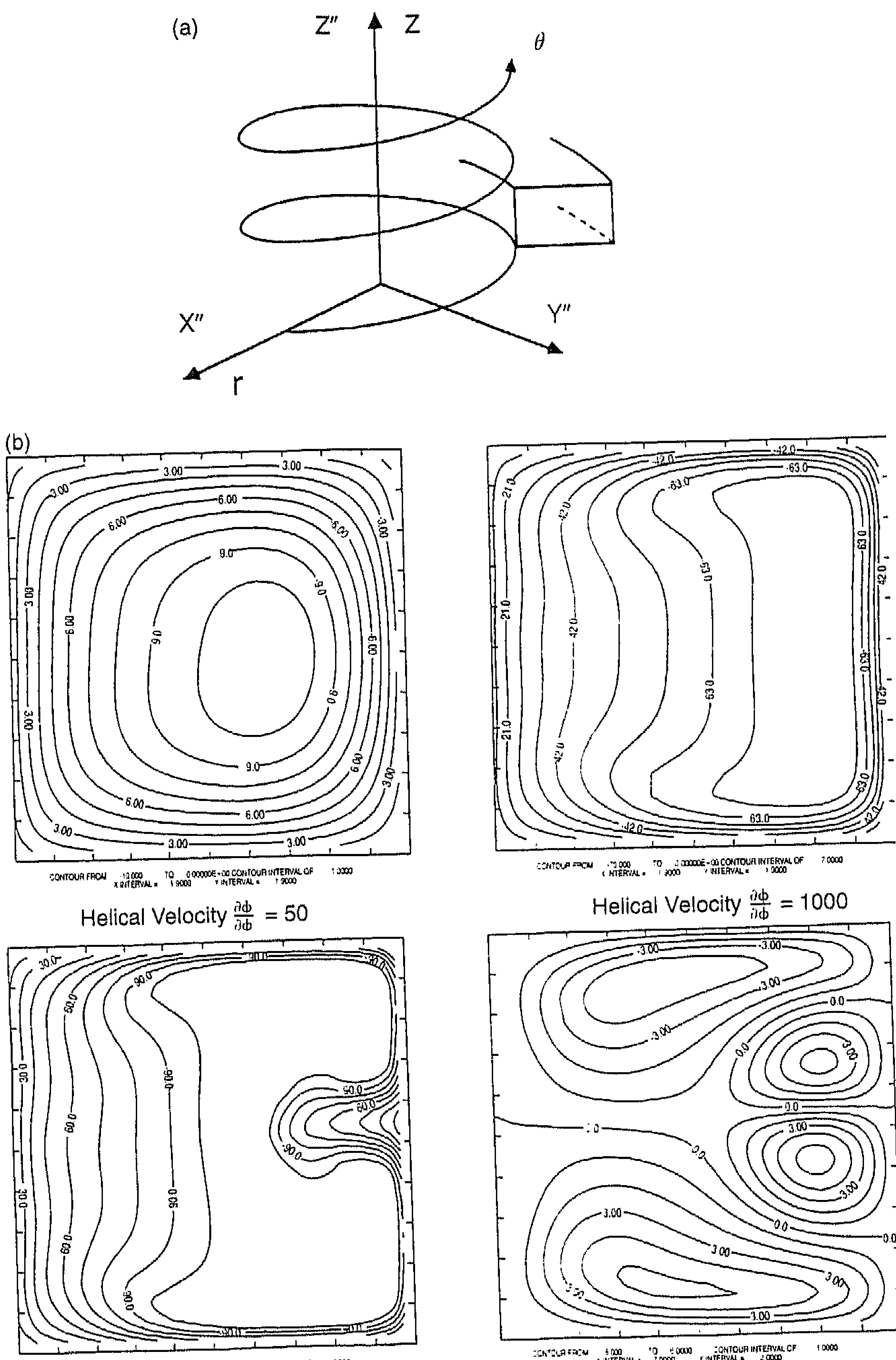

$$
\text { Helical Velocity } \frac{i \mathrm{ith}}{\mathrm{i} \phi}=2300
$$

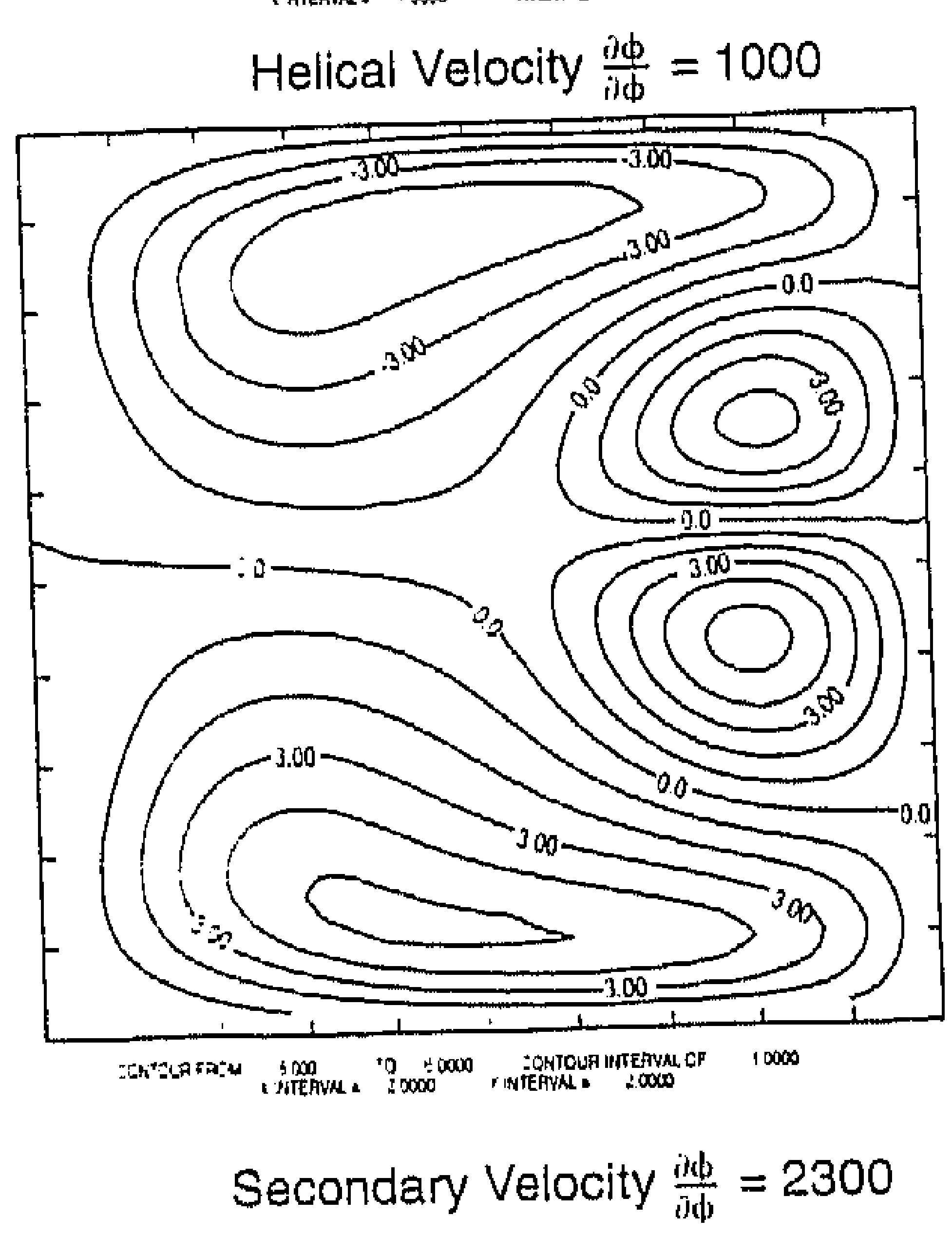

Fig 8. (a) Helical coordinate system and (b) effect of the pressure gradient in the circumferential direction $(\partial \phi \partial \theta)$ on secondary thow structure. [From Wang, J.W., and Andrews, J.R.G., Numerical simulation of flow in helical ducts. AIChE J. 41(5), 1071 (1995). Reproduced with permission of the American Institute of Chemical Engineers. Copyright 1995 AIChE. All rights reserved.] 
secondary flow changes significantly. These calculations are highly relevant to tubular reactors where the "tube" has been wrapped in the form of a coil due to space limitations. The occurrence of secondary flow can also be used to promote transport of mass and/or heat to the tube wall and in this case it is important that a reliable prediction of the associated enhancement factors can be made.

Further examples of recent applications of the finite element method can be found in Targett et al. (1995) who studied flow through curved rectangular channels of large aspect ratio using the FEM-based FIDAP code. They also compared their computational results with experimental data obtained from visualization experiments and found good agreement between theory and experiment.

b. Laminar Flow around Bluff Bodies Inserted in Channels. In the literature numerous computational studies can be found that deal with the flow around bluff bodies. Anagnostopoulos and Iliadis (1996) used a FEM to study the vortex shedding behind a cylinder positioned in a channel, whereas Xu and Michaelides (1996) performed a numerical study of the flow over an ellipsoidal object inside a cylindrical tube. The extension of such calculations to systems that involve arrays of cylinders or "tube bundles" as encountered in many heat transfer applications is in principle possible but they are very CPU intensive. Figure 9 shows as an itlustration of the Karman vortex street formed behind a cylinder (diameter $d$ ) positioned in a channel (width 2h) (Anagnostopoulos and lliadis, 1996) at a Reynolds number of 106. From the pattern of the solid lines (equivorticity lines) shown in this figure the vortical structures behind the cylinder can be clearly recognized. In addition, the increasing influence of the channel walls on the vortical structures with increasing $d / h$ ratio can be inferred from Fig. 9.

c. Laminar Flow in Stirred Vessels. The blending or mixing of highly viscous (miscible) liquids is encountered in a variety of industrial operations and has been studied computationally by, among others, Abid et al. (1992). They studied the effect of agitator geometry on the flow patterns generated in a stirred vessel containing a very viscous liquid and performed both $2 \mathrm{D}$ and $3 \mathrm{D}$ numerical simulattions. Especially in the case of very viscous fluids, which are often not transparent and therefore not accessible to advanced experimental techniques such at laser Doppler anemometry (LDA), a CFD approach can be very beneficial to generate useful data, provided that some kind of experimental validation of the computational strategy has been undertaken. This is especially important for these types of flows where (considerable) simplifications of the agitator geometry are often made in the computations.

d. Concluding Remarks. The simulation of processes involving 3D laminar fluid flow and associated phenomena such as mass and heat transport and chemical 

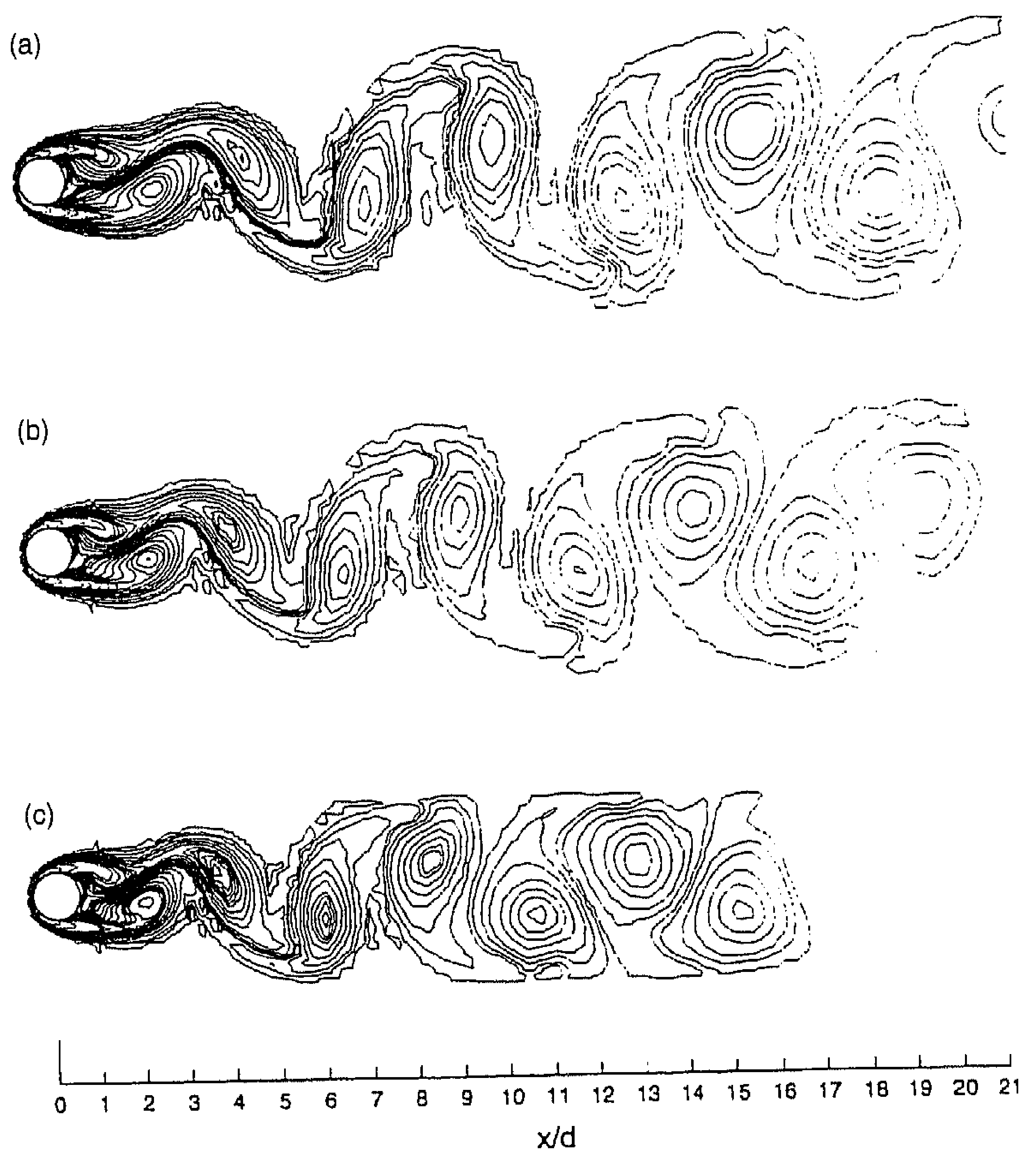

Fig. 9. Karman vortex street formed behind a cylinder (diameter $d$ ) positioned in a channel (width $2 / 2$ ) at a Reynolds number of 106 . [From Anagnostopoulos, P.. and Iliadis. G. Numerical study of the blockage effect on viscous flow past a circular cylinder. Int. J. Num. Methods Fluids 22. 1061 (1996). Copyright John Wiley \& Sons Limited. Reproduced with permission.]

transformation can nowadays be performed with great accuracy. The main difficulties that can emerge here are due to (1) the use of insufficiently refined computational grids to resolve all relevant details of the flow. (2) the accurate treatment of moving boundaries as encountered in agitated vessels, and (3) the use of inappropriate numerical methods which cause severe numerical diffusion.

\section{Turbulent Flows}

For systems involving turbulent flows accurate modeling is much more complicated in comparison with those involving laminar flow and here it is considered very important to interpret and use the computational results with great care, especially when one deals with turbulent flows in complex geometries. Whenever 
possible a (limited) comparison with experimental data should be made to assess the correctness of the computations. This state of the art is due to the fact that our understanding of and our capability to predict turbulent fluid flow phenomena is unfortunately still limited as pointed out by Banerjee (1991) in a very lucid manner. Other reviews on the modeling and numerical simulation of turbulent flows can be found in Rogallo and Moin (1984), Launder (1991), and Reynolds (1991),

In studies that involve the CFD analysis of turbulent fluid flow, the $k$ - $\epsilon$ model is most frequently used because it offers the best compromise between width of application and computational economy (Launder, 1991). Despite its widespread popularity the $k-\epsilon$ model, if used to generate an isotropic turbulent viscosity, is inappropriate for simulation of turbulent swirling flows as encountered in process equipment such as cyclones and hydrocyclones (Hargreaves and Silvester, 1990) and more advanced turbulence models such as the ASM or the RSM should be considered. Because these models are computationally much more demanding and involve an increased number of empirical parameters compared to the $k-\epsilon$ model, other strategies have been worked out (Boysan et al., 1982; Hargreaves and Silvester, 1990) to avoid the isotropic nature of the classical $k-\epsilon$ model.

Due to advances in computer technology and numerical solution techniques both DNS and LES have become feasible. Although DNS is still limited to relatively low Reynolds numbers, it is a very powerful tool for understanding turbulent flow structure and a tool to generate databases of turbulent flows which can be used, for example, to validate conventional turbulence models such as $k-\epsilon$ or Reynolds stress models. A good example in this respect is the study conducted by Hrenya et al. (1995) who tested 10 turbulence models against DNS results and experimental data. Hrenya et al. found that the model proposed by Myong and Kasagi (1990) showed the best overall performance in predicting fully developed, turbulent pipe flow. Eggels (1994) performed both DNS and LES simulations of turbulent pipe flow and also made a detailed comparison with experimental data obtained with a digital particle image velocimetry (DPIV) technique developed by Westerweel (1993).

The extension of this approach to higher Reynolds numbers and more complex geometries is very important and deserves further attention in the future but depends critically on the future advances in computer hardw'are and numerical solution methods. As far as LES or partially resolved simulations are concerned, similar limitations exist although these type of simulations can be carried to (much) higher Reynolds numbers. DNS can also be used to obtain insight in turbulence producing mechanisms as shown in the study of Lyons et al. (1989).

A flow configuration of particular interest to the chemical engineer is the (baffled) stirred vessel and very significant efforts have been documented in the literature (Ranade and Joshi, 1990a,b), Kresta and Wood, 1991) to compute the flow patterns inside the vessel using CFD models. Here models with a varying degree of sophistication both from a physical and numerical point of view have been 
used. In most studies the $k-\epsilon$ model was used with standard values for the turbulence parameters to compute the turbulent flow patterns despite the fact that these parameter values were obtained for (much) simpler geometries. Another principal difficulty in modeling turbulent flows in stirred vessels is the accurate description of the impeller geometry, whereas the resolution of the vortical structures behind the baffles is also problematic due to the coarse grids which are commonly employed in the simulations. A possible approach to describe the impeller geometry in more detail is the application of so-called sliding meshes where two meshes are applied: one mesh for the central impeller region, which rotates with the speed of the impeller, and another one for the exterior region. which is fixed in space. An alternative approach is the use of a single moving deforming mesh as reported by Perng and Murthy (1992). Here the grid is attached to the impeller and moves with it and as a consequence mesh deformation occurs.

CFD has also been applied to analyze the flow patterns in a special countercurrent solvent extraction column (Angelov et al., 1990). They used a singlephase flow representation and a $k-\epsilon$ turbulence model to compute the flow patterns in a periodic structure of the column. Validation of the computational results was achieved by applying LDA to obtain experimental data on the velocity profiles. CFD is a very useful tool here because the optimization of the performance of the extraction column from a geometrical point of view can be achieved with relative ease in comparison with a pure empirical strategy.

In the last decade very significant progress has been made in modeling turbulent fluid flow. There remain, however, very significant problems of which. in addition to the problems mentioned in the previous section, we would like to mention the following problem areas: (1) availability of accurate turbulence models which can be used with confidence in complex geometries while at the same time the computational cost should be acceptable and (2) availability of DNSgenerated databases to validate semiempirical turbulence models.

\section{Complex Rheology}

In several industrial processes the engineer has to deal with non-Newtonian fluids of which food processing and production processes involving glues, colors. polymer solutions, and pure polymers are well-known examples.

The simulation of non-Newtonian fluid flow is significantly more complex in comparison with the simulation of Newtonian fluid How due to the possible occurrence of sharp stress gradients which necessitates the use of (local) mesh refinement techniques. Also the coupling between momentum and constitutive equations makes the problem extremely stiff and often time-dependent calculations have to be performed due to memory effects and also due to the possible occurrence of bifurcations. These requirements explain the existence of specialized (otten FEM-based) CFD packages for non-Newtonian flow such as POLYFLOW. 
Both FVM and FEM based packages have been applied successfully to study non-Newtonian fluid flow.

The progress and challenges in computational rheology have been recently reviewed by Keunings (1990). In literature viscoelastic liquids have received particular attention (Crochet, 1987; Boger, 1987; Keunings, 1990: Yoo and Na, 1991; Van Kemenade and Deville, 1994; Mompean and Deville, 1996) due to the fact that many industrially relevant non-Newtonian materials such as polymer solutions and polymers exhibit viscoelastic behavior. In addition the strong interplay between modeling and mathematical and numerical considerations makes this particular field fascinating but also very complex, which requires good cooperation among rheologists, mathematicians, and CFD experts.

Boger (1987) has reviewed viscoelastic flows through contractions and has pointed out that three-dimensional and time-dependence characteristics have to be taken into account for flows at high Weissenberg number We defined by:

$$
\mathrm{We}=\frac{\lambda V}{L}
$$

where $\lambda$ is the relaxation time of the fluid and $L / V$ the characteristic inertial time of the flow based on a reference length $L$ and reference velocity $V$.

Examples of CFD applications involving non-Newtonian flow can be found, for example, in papers by Keunings and Crochet (1984), Van Kemenade and Deville (1994), and Mompean and Deville (1996). Van Kemenade and Deville used a spectral FEM and experienced severe numerical problems at high values of the Weissenberg number. In a later study Mompean and Deville (1996) could surmount these numerical difficulties by using a semi-implicit finite volume method.

Similar to the role that DNS and discrete particle models (see Section IV,B.3) might play in the development of improved turbulence models, which can be used in engineering applications, and closure laws for gas-solid continuun models. Brownian dynamics (BD) should be mentioned as al powerful tool to develop closure models for non-Newtonian fluids (Brady and Bossis, 1988).

The CFD-analysis of non-Newtonian flow has made progress in the last decade but the extension of the calculations to other types of non-Newtonian fluid flow and more complex geometries is highly desirable from the perspective of the chemical engineer.

\section{Mixing and Chemically Reactive Flows}

By the very nature of the profession, the chemical engineer has to deal very frequently with chemically reactive flows in various types of single-phase and multiphase reactors. Before the advent of CFD he or she typically had to use 
highly idealized and approximate solution strategies supplemented with empirical information to obtain solutions for practical problems. A very well-known approach in this connection is the combined use of macromixing and micromixing models to predict the performance (i.e., conversion and/or selectivity) of a chemical reactor. Here, with the aid of stimulus-response experimental techniques, information on the residence time distribution (RTD) is obtained that is subsequently used to devise a macromixing model whereas a postulated micromixing model is invoked to account for the finite rate with which segregated fluid parcels eventually achieve mixing at the molecular level (i.e., segregation decay) to permit the occurrence of a chemical transformation. During the last 30 years a host of micromixing models has been proposed in the literature of which the coalescence dispersion (CD) model (Curl, 1963; Spielman and Levenspiel, 1965), the interaction by exchange with the mean model (IEM) (Villermaux and Devillon, 1972), and the general micromixing model (GMM) (Villermaux and Falk, 1994) should be mentioned as typical examples. For further references in this area, the interested reader is referred to the reviews by Ottino (1994), Villermaux and Falk (1996), and Fox (1996). It should also be mentioned here that very important contributions toward a better understanding and description of chemically reactive flows in general and related CFD work in particular have been made by the mechanical engineering community with combustion research as an important driver. For a review from this perspective we refer to Correa and Shyy (1987).

Until 10 to 15 years ago the combined approach of macromixing and micromixing models was very widely used in the field of CRE but gradually CFDbased strategies have replaced the first mentioned strategy. In this respect it should be noted that this change also introduced big conceptual differences because the traditional CRE approach is usually formulated in the "age" space of fluid parcels whereas in CFD approaches a Eulerian framework is often adopted. Subsequently a brief overview of CFD-based approaches for reacting flows is presented and the current limitations are also indicated.

a. Direct Numerical Simulation. Direct numerical simulation (DNS) has also been applied to chemically reactive flows (Givi and McMurtry, 1988; Leonard and Hill, 1988; McMurtry and Givi, 1989) but due to current limitations in computer capacity it is very difficult to apply DNS at high Reynolds and Schmidt numbers. Since such situations are frequently encountered in chemical process engineering where in addition the geometry is often (very) complex, DNS is not considered a practical tool for the simulation of industrial reactors. However, it is a very interesting research tool to study chemistry-turbulence interactions and to generate databases which can be used for the verification of mixing closure laws used in process engineering models. 
b. Large Eddy Simulation. Although LES can be applied at (much) higher Reynolds numbers and has the potential to predict accurately time-dependent macromixing patterns in process equipment with complex geometries (i.e., stirred tanks), its principal weakness lies in the fact that turbulence-chemistry interaction at the subgrid scale (SGS) (i.e., the scale smaller than the smallest resolved scale) has to be modeled. These SGS models themselves are computationally demanding, which hampers the combined use with LES, which is also computationally intensive.

c. Moment Methods. Turbulent reacting flows are frequently encountered in the chemical process industry and very often (i.e., in most commercial CFD codes) the theoretical description of these flows is based on the Reynolds averaged Navier-Stokes (RANS) equations (Brodkey and Lawelle, 1985; Launder, 1991). The consequence of this approach is the necessity to specify closure laws for the Reynolds stresses and fluxes of scalar quantities (i.e., mass or molar fluxes) and most importantly the average reaction rate. This last term is the most difficult term due to its nonlinearity, and its treatment depends on the magnitude of the Damköhler number Da (Fox, 1996). For chemically reactive flows a distinction should be made between three important classes, which can be classified in terms of the Damköhler number $\mathrm{Da}=t_{\mathrm{m}} / t_{\mathrm{r}}$ where $t_{\mathrm{m}}$ and $t_{\mathrm{r}}$, respectively, represent a characteristic time scale for turbulent micromixing and a characteristic time scale for chemical transformation:

- $D a<<1$ : flows where the time scale for chemical conversion $t_{\mathrm{r}}$ is relatively large compared to the time scale for turbulent micromixing $t_{\mathrm{m}}$

- $D a>>1$ : flows where the time scale for chemical conversion $t_{\mathrm{r}}$ is relatively small compared to the time scale for turbulent micromixing $t_{\mathrm{m}}$

- $D a \approx 1$ : flows where the time scale for chemical conversion $t_{\mathrm{r}}$ is comparable in magnitude to the time scale for turbulent micromixing $t_{\mathrm{m}}$.

For the first two cases $\mathrm{Da}<<1$ (slow reactions) and $\mathrm{Da}>>1$ (very fast reactions) adequate closure models are available in many commercial CFD codes. For the third case, where the time scale for chemical conversion approximately equals the time scale for turbulent micromixing, moment methods are inappropriate and other methods should be used. In this situation the reactor performance may be significantly affected by mixing efficiency. Here the engineer is faced with the difficult problem of predicting the overall conversion and/or selectivity of the chemical process. In the last three decades this problem has received considerable attention in three scientific areas, namely, chemical reaction engineering, fluid mechanics and combustion, and various approaches have been followed.

d. Micromixing Models. Micromixing models offer the advantage that the chemical reaction rate expression is treated in an exact manner but they suffer from the 
fact that highly idealized descriptions of the fluid dynamics (backed up by empirical information) are used. This latter point certainly constitutes a major weakness of micromixing models, however, by incorporating micromixing models in RANS models this disadvantage can be partly overcome. For complex inhomogeneous flows this approach again introduces significant difficulties and recourse should be made to methods that treat the chemistry and turbulence and their mutual interaction on a more fundamental basis.

e. Probability Density Function Methods. Probability density function (PDF) methods combine the strong points of exact treatment of the chemical reaction term and second-order turbulence closure (Fox, 1996). PDF methods make use of a probability density distribution function $f$ which can take the form of a presumed function or a transport equation which has to be solved simultaneously with the fluid flow equations. Once the function $f$ is known, the expected value $\langle c\rangle$ of an arbitrary function $c$ (including, for example, the chemical reaction rate) can be computed as follows:

$$
<c>=\int c f d \vec{x}
$$

where the integration has to be performed over all possible values of all elements constituting the vector $\bar{x}$. This vector usually consists of the three velocity components and all concentrations (or mass fractions, etc.) of the species involved in the chemical transformation, where all quantities are evaluated at a fixed point. Full PDF methods have proven very successful in the description of complex turbulent reacting flow but, unfortunately, due to limitations in computer hardware, at present full PDF methods are limited to the analysis of turbulent reacting flows involving three spatial variables and for five to six chemical species with arbitrary chemical kinetics. This limitation is due to the fact that full PDF methods typically make use of a so-called "chemical look-up table" which contains information of precomputed composition changes due to chemical conversion. For further details on full PDF methods the interested reader is referred to Pope (1994) and the excellent review paper by Fox (1996). These classes of chemically reactive flows are briefly discussed together with some illustrative applications.

f. Slow Chemical Reactions $D a \ll 1$. De Saegher et al. (1996) developed a comprehensive 3D reactor model for thermal cracking of hydrocarbons in internally finned tube reactors where both tubes with longitudinal and helicoidal tins were studied. Their model combines detailed descriptions of the 3D turbulent fluid flow in tubes with a complex internal geometry, endothermic multispecies chemical conversion, and heat transfer. Despite the fact that thermal cracking processes of hydrocarbons typically involve high temperatures, they could show that the ratio of $t_{\mathrm{r}}$ and $t_{\mathrm{m}}$ was sufficiently small to justify the neglect of 
turbulence-chemistry interactions. Although the gas temperatures did not differ much between tubes with longitudinal fins and tubes with helicoidal fins, the nonuniformity of the process gas over the tube cross section was much more pronounced for tubes with longitudinal fins. Traditional reactor models, which assume plug flow, are inadequate to predict the performance of cracking furnaces employing such new internal configurations of the tubes. Moreover these simple reactor models are not able to predict the circumferential nonuniform coke deposition in the tubes.

g. Very Fast Chemical Reactions Da $\gg 1$. Very fast reactions are encountered, for example, in the domain of acid-base chemistry. Pipino and Fox (1994), for example, studied acid-base (i.e., $\mathrm{HCl}-\mathrm{NaOH}$ ) neutralization in a laboratory tubular reactor and used two different PDF approaches to describe quantitatively the effect of turbulent mixing on the chemical conversion. Their first description was based on the Lagrangian joint PDF of velocity and composition, whereas the second description was based on the Eulerian composition PDF. Pipino and Fox used the CFD package FLUENT to obtain the mean velocity field and the turbulence quantities and proposed a new model for molecular mixing that explicitly accounts for relaxation of the concentration spectrum. They used a spectroscopic method to determine the absorbance of the reacting mixture, containing phenol red as $\mathrm{pH}$ indicator, along the reactor axis. In their paper they reported good agreement between simulation results and experimental data, provided that their new molecular mixing model was invoked in the simulations. The study of Pipino and Fox clearly demonstrates that PDF simulations are a powerful tool for simulating mixing-sensitive chemical transformations in a turbulent flow field. The extension to (significantly) more complex reaction schemes and geometries is considered highly desirable.

h. Fast Chemical Reactions $D a \approx 1$. A very difficult and challenging (both from a theoretical and a computational point of view) problem arises when the time scale for the chemical reaction is comparable to the time scale for (turbulent) mixing of the fluid. A well-known example of this class is encountered in the diazocoupling of 1-naphtol (A) and diazotized sulfanilic acid (B) in an alkalinebuffered solution:

$$
\begin{aligned}
& \mathrm{A}+\mathrm{B} \frac{k_{1}}{\rightarrow} \mathrm{R} \\
& \mathrm{R}+\mathrm{B} \underset{\frac{k_{2}}{\rightarrow}}{\rightarrow} \mathrm{S}
\end{aligned}
$$

In this case $k_{1} \gg k_{2} \approx 1 / t_{\mathrm{m}}$ and consequently full PDF methods are required to describe accurately the turbulence-chemistry interactions. Tsai and Fox (1994) 
have reported full PDF simulations for the above-mentioned chemical system and have compared their simulation results with experimental data reported by Li and Toor (1986).

i. Concluding Remarks. With respect to chemically reactive flows it can be stated that significant progress has been made toward a fundamental CFD-based description of these systems. Particularly promising are the so-called full PDF models (Pope, 1994; Fox, 1996). However, the industrial demands are in the area of (much) more complex reaction schemes involving a large number of species and reactions, very viscous fluids, non-Newtonian flow, and last but not least multiphase flows. In these areas much (CFD) work remains to be done. Keep in mind, however. that in many processes encountered in chemical engineering detailed information on the intrinsic chemical kinetics is lacking and therefore a good balance should be maintained between (very) detailed modeling of turbulence and turbulence-chemistry interactions and available information on the rate of the chemical transformations. In addition the availability of nonintrusive experimental techniques to obtain whole field concentration distributions is critical for the future development of this important class of CRE problems (see Section V).

\section{B. Multiphase SySTEMS}

In many processes encountered in industrial practice, multiphase flows are encountered and in general it can be stated that, due to the inherent complexity of such flows, general applicable models and related CFD codes are nonexistent. The reason for this relatively unsatisfactory state of the art is due to the following causes:

- Many types of multiphase flow exist (i.e., gas-liquid, gas-solid, liquidliquid, gas-liquid-solid) where within one type of flow several possible flow regimes exist. In Fig. 10 (Ishii, 1975) a classification is given for two-phase flow.

- The detailed physical laws and correct mathematical representation of phenomena taking place in the vicinity of interfaces (coalescence, breakup, accumulation of impurities) are still largely undeveloped.

Very often multiphase flow systems show inherent oscillatory behavior that necessitates the use of transient solution algorithms. Examples of such flows are encountered in bubbling gas-fluidized beds, circulating gas-fluidized beds, and bubble columns where, respectively, bubbles, clusters, or strands and bubble plumes are present that continuously change the flow pattern. 


\begin{tabular}{|c|c|c|c|c|}
\hline Class & $\begin{array}{l}\text { Typical } \\
\text { regimes }\end{array}$ & Geometry & Configuration & Examples \\
\hline \multirow{3}{*}{ 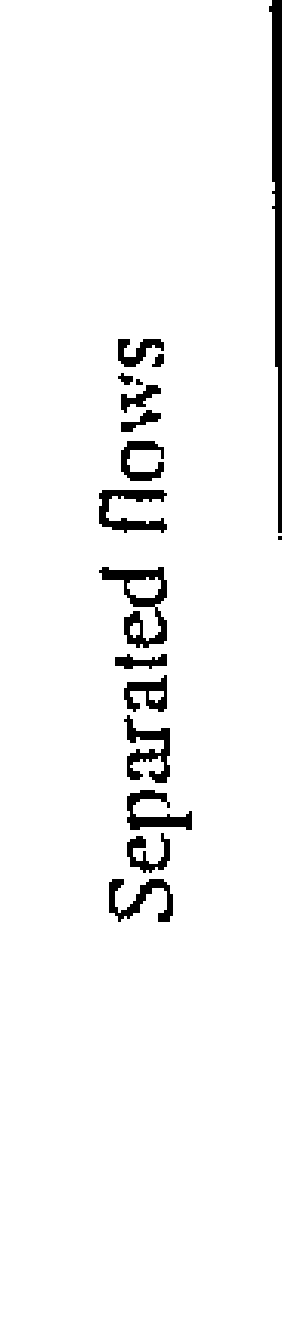 } & Film flow & 雨 & $\begin{array}{l}\text { liquid film in gas } \\
\text { gas film in liquid }\end{array}$ & $\begin{array}{l}\text { film cooling } \\
\text { film boiling }\end{array}$ \\
\hline & Annular flow & 羿\} & $\begin{array}{l}\text { liquid core and gas film } \\
\text { gas core and liquid film }\end{array}$ & $\begin{array}{l}\text { film boiling } \\
\text { condensers }\end{array}$ \\
\hline & Jet flow & हो & $\begin{array}{l}\text { liquid jet in gas } \\
\text { gas jet in liquid }\end{array}$ & $\begin{array}{l}\text { atomization } \\
\text { jet condenser }\end{array}$ \\
\hline \multirow{4}{*}{ 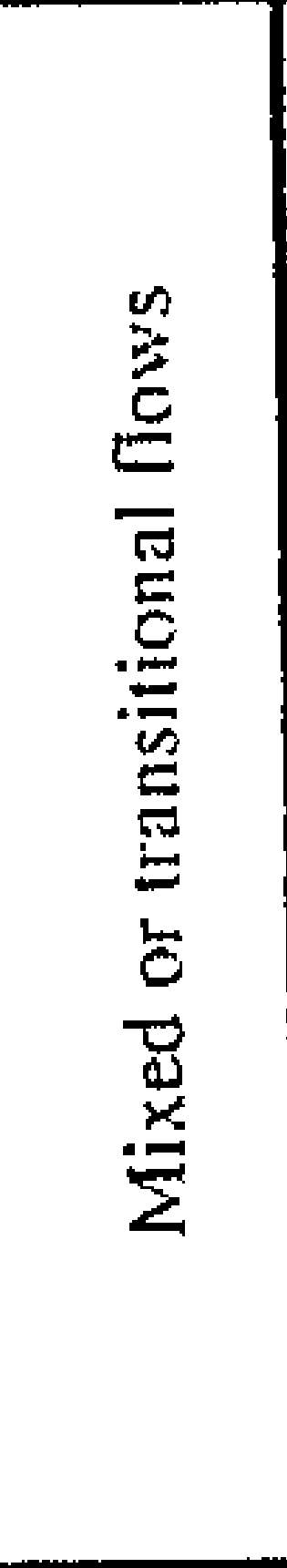 } & Slug (plug) flow & 3 & gas pocket in liquid & $\begin{array}{l}\text { sodium boiling in forced } \\
\text { convection (liquid metal } \\
\text { fast breeder reactor) }\end{array}$ \\
\hline & $\begin{array}{l}\text { Bubbly annular } \\
\text { flow }\end{array}$ & 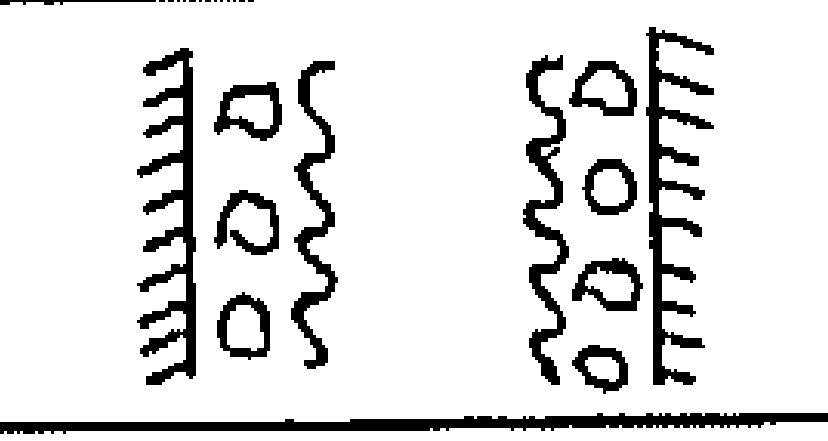 & $\begin{array}{l}\text { gas bubbles in liquid } \\
\text { film with gas core }\end{array}$ & $\begin{array}{l}\text { evaporators with wall } \\
\text { nucleation }\end{array}$ \\
\hline & $\begin{array}{l}\text { Droplet annular } \\
\text { flow }\end{array}$ & $\exists\left\{_{300\}}=0\right.$ & $\begin{array}{l}\text { gas core with droplets } \\
\text { and liquid film }\end{array}$ & steam generator \\
\hline & $\begin{array}{l}\text { Bubbly droplet } \\
\text { annular flow }\end{array}$ & $\begin{array}{l}\text { Jos:0 sok } \\
\text { J050050E } \\
\text { J05:050 }\end{array}$ & $\begin{array}{l}\text { gas core with droplets } \\
\text { and liquid film with } \\
\text { gas bubbles }\end{array}$ & $\begin{array}{l}\text { boiling nuclear } \\
\text { reactor channel }\end{array}$ \\
\hline \multirow{3}{*}{ 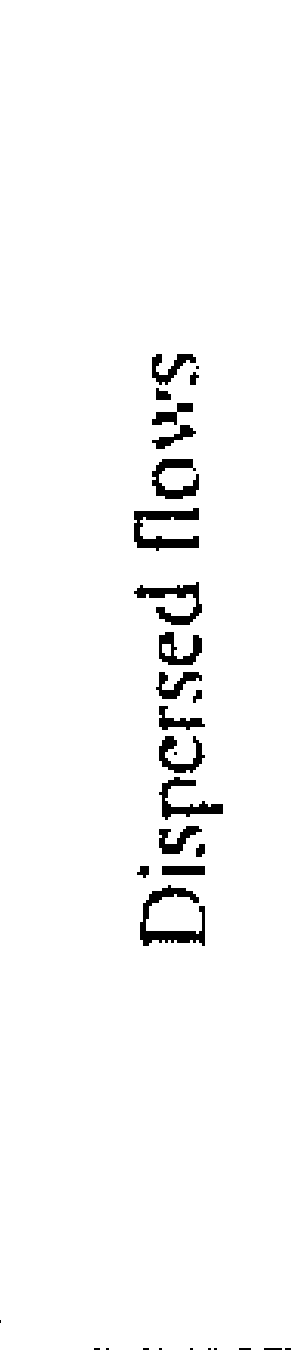 } & Bubbly flow & 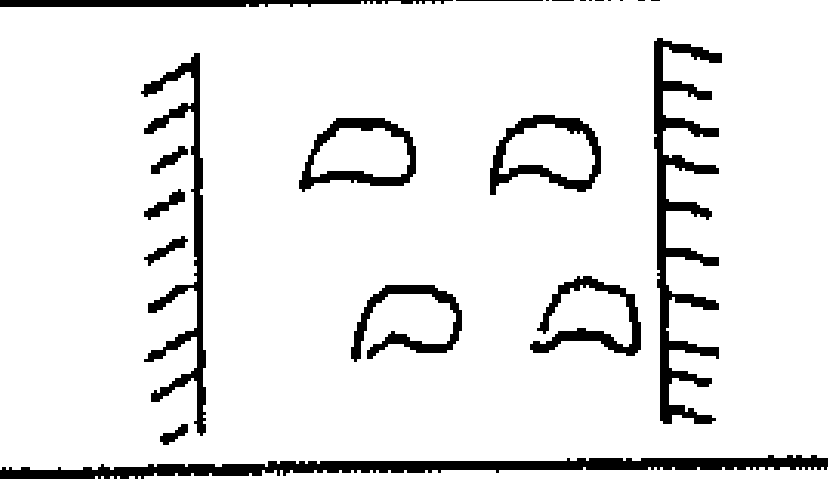 & gas bubbles in liquid & bubble columns \\
\hline & Droplet flow & $\exists=10 \mathrm{E}$ & liquid droplets in gas & spray towers \\
\hline & Particulate flow & $\exists \cdot \because E$ & $\begin{array}{l}\text { solid particles in gas } \\
\text { solid particles in liquid }\end{array}$ & $\begin{array}{l}\text { gas fluidized beds } \\
\text { liquid fluidized beds }\end{array}$ \\
\hline
\end{tabular}

Fig. 10. Flow regime classification for two-phase flow (Adapled from Ishii (1975)).

Prior to the discussion of the progress in the CFD analysis of multiphase flows, first some general requirements for modeling multiphase systems are mentioned:

- Regime characterization and flow regime transition

- Spatial distribution of the phases

- Sensitivity of system behavior for physicochemical parameters

- Prediction of effect of internals 


\section{Gas-Liquid (G-L) Systems}

Gas-liquid systems are encountered very frequently in a variety of industrial applications. For example, the production of crude oil and natural gas involves the transportation of a gas and a liquid phase in pipes. Although very significant efforts have been made to arrive at a fundamental description and subsequent CFD modeling of these type of flows, unfortunately the progress is still very limited and the engineer, faced with the solution of practical problems, very often has to resort to semiempirical methods. This state of the art is mainly due to the fact that numerous flow regimes, with their specific hydrodynamic characteristics, can prevail.

Gas-liquid systems of particular interest to the chemical engineer are encountered in bubble columns, spray columns, air lift, falling film, and stirred tank reactors. Usually the form of these reactors corresponds to that of vessels or columns. From the perspective of the chemical engineer, who is concerned with the conversion and selectivity of chemical transformations, it is of utmost importance that an intensive contact between a gas and a liquid be achieved and therefore very often one phase is continuous whereas the other is disperse. Therefore, the interfacial area and the size of the disperse phase elements constitute very important aspects of CFD modeling of these types of systems.

As mentioned earlier many two-phase gas-liquid flows are highly dynamic and this property requires the use of dynamic simulation methods. A well-known example of such a type of flow is encountered in bubble columns where recirculating flow structures are present which are not stationary but which continuously change their size and location in the column. Due to their frequent application in chemical reaction engineering and their relatively simple geometry, CFD analyses of bubble columns have received significant attention and are discussed in more detail here.

a. Bubble Columns. Both two-fluid Eulerian models (Gasche et al., 1990; Torvik and Svendsen, 1990; Svendsen et al., 1992; Hjertager and Morud, 1993: Sokolichin and Eigenberger, 1994) and mixed Eulerian-Lagrangian models (Trapp and Mortensen, 1993; Lapin and Lübbert, 1994; Devanathan et al., 1995; Delnoij et cll., 1997a) have been used to study gas-liquid two-phase flow. The general advantages of mixed Eulerian-Lagrangian models in comparison with completely Eulerian models are as follows: (1) Phenomena such as bubble breakup and coalescence can be implemented in a more direct way, and (2) the computational smearing or numerical diffusion can be significantly reduced (see, for example, Lapin and Lübbert, 1994). However, by applying higher order numerical schemes for the evaluation of the convective fluxes instead of the often used first-order UPWIND numerical scheme, the computational smearing of the 
Eulerian approach can be reduced significantly (Sokolichin et al., 1997). Especially for dispersed flows with a high volume fraction of the dispersed phase, the increased computational requirements of mixed Eulerian-Lagrangian approaches should be mentioned as a disadvantage.

Figure 11 shows as an example some typical computational results obtained by Lapin and Lübbert with a mixed Eulerian-Lagrangian approach. This figure shows the combined results for the liquid phase velocity pattern (left) and the bubble positions (right) in a wafer column (diameter, $1.0 \mathrm{~m}$; height, $1.5 \mathrm{~m}$ ) where the bubbles are generated uniformly over its entire bottom. The simulation was started at $T=0$ (s) with a bubble-free liquid. Initially the bubble clusters were found to rise in the liquid in the shape of a plane front but after approximately $2 \mathrm{~s}$ the front becomes unstable and attains a wavy shape. After $5 \mathrm{~s}$ a considcrable instability has developed near the wall, which eventually leads to macroscopic flow instability (i.e., very irregular flow structure with large density differences). However, about $70 \mathrm{~s}$ after startup fairly regular flow patterns are established where several circulation cells can be recognized which were found to change their size and location continuously during the rest of the simulation. The flow in the bubble column is thus inherently instationary and a true steady-state flow pattern does not exist. Lapin and Lübbert stress in their paper the use of a sufficiently refined computational mesh in order to avoid excessive computational smearing of the resolved flow structures. With respect to the model presented by Lapin and Lübbert it should be mentioned here that their approach is essentially a pseudo-Lagrangian one due to the fact that they track bubble clusters instead of individual bubbles. Moreover, their model does not account for bubble-bubble interactions and momentum exchange between the bubbles and the liquid. The induced flow in the liquid phase is thus in fact assumed to be due to buoyancy effects only, which is probably a reasonable assumption in this type of flow. A more refined model in this respect has been developed by Delnoij et al. (1997a) and is discussed in more detail in Section VI.

Kumar et al. (1995) used the CFDLIB code developed at Los Alamos Scientific Laboratory to simulate the gas-liquid flow in bubble columns. Their model, which is based on the Eulerian approach, could successfully predict the experimentally observed von Karman vortices (Chen et al., 1989) in a 2D bubble column with large aspect ratio (i.e., ratio of column height and column diameter).

Besides the modeling of bubble columns with either Eulerian or mixed Eulerian-Lagrangian models the dynamics of individual bubbles has also been studied extensively in literature using various CFD approaches (Ryskin and Leal, 1984a.b.c: Tomiyama et al.. 1993; and Hoffman and van den Bogaard, 1995). Ryskin and Leal solved the Navier-Stokes equations for the liquid flowing around a deformable bubble where the orthogonal curvilinear coordinate system was constructed in accordance with the shape which the bubble attained during its rise through the liquid, whereas Tomiyama et al. and Hoffman et al., respectively, used the VOF method (Hirt and Nichols, 1981) and the finite element based 
a: $\mathrm{T}=6.7[\mathrm{~s}]$

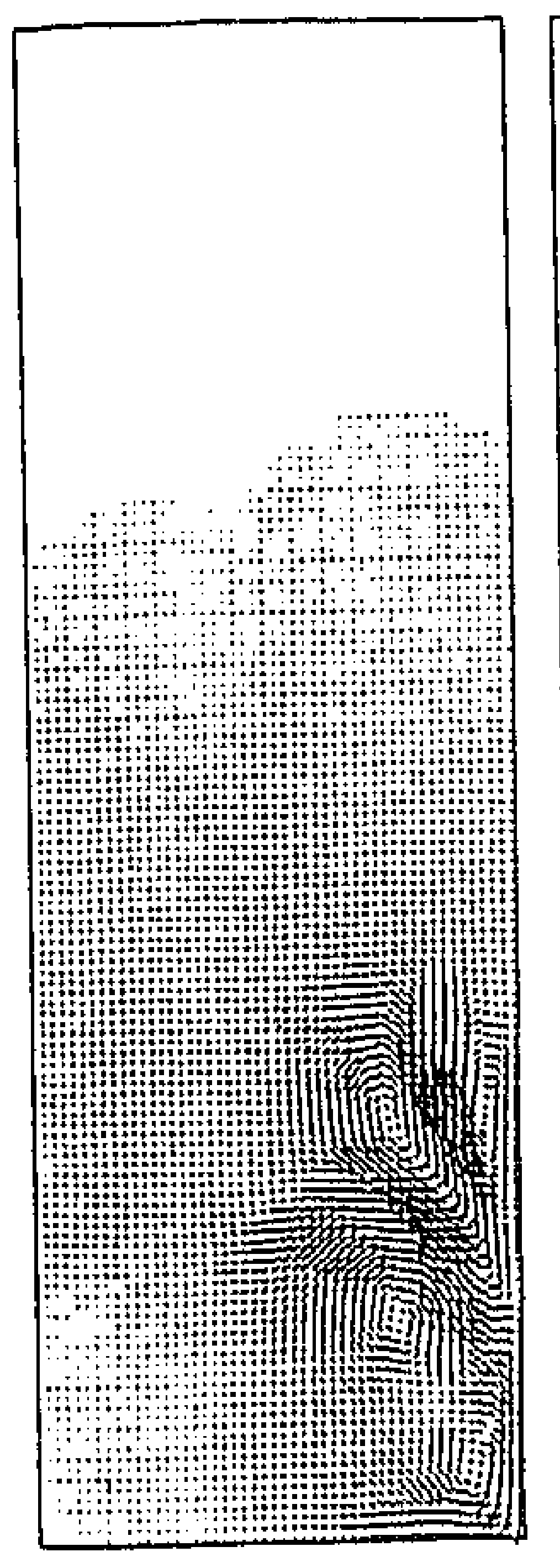

c: $\mathrm{T}=69.7[\mathrm{~s}]$

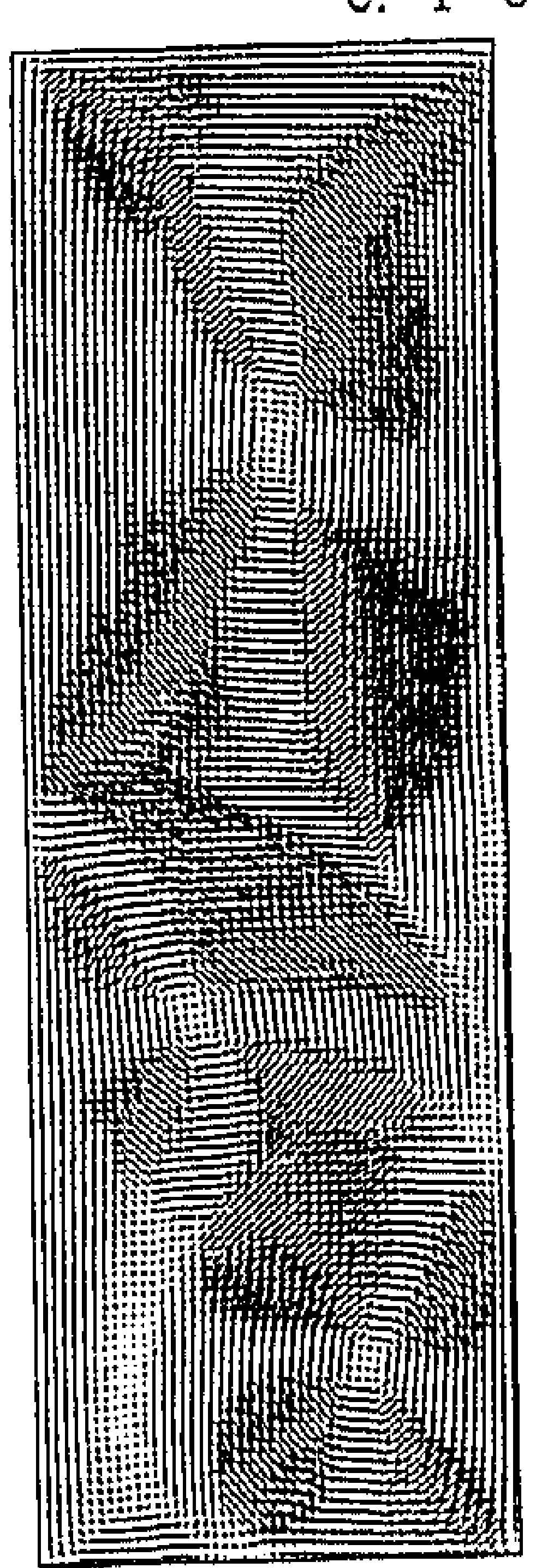

b: $\mathrm{T}=19.9[\mathrm{~s}]$
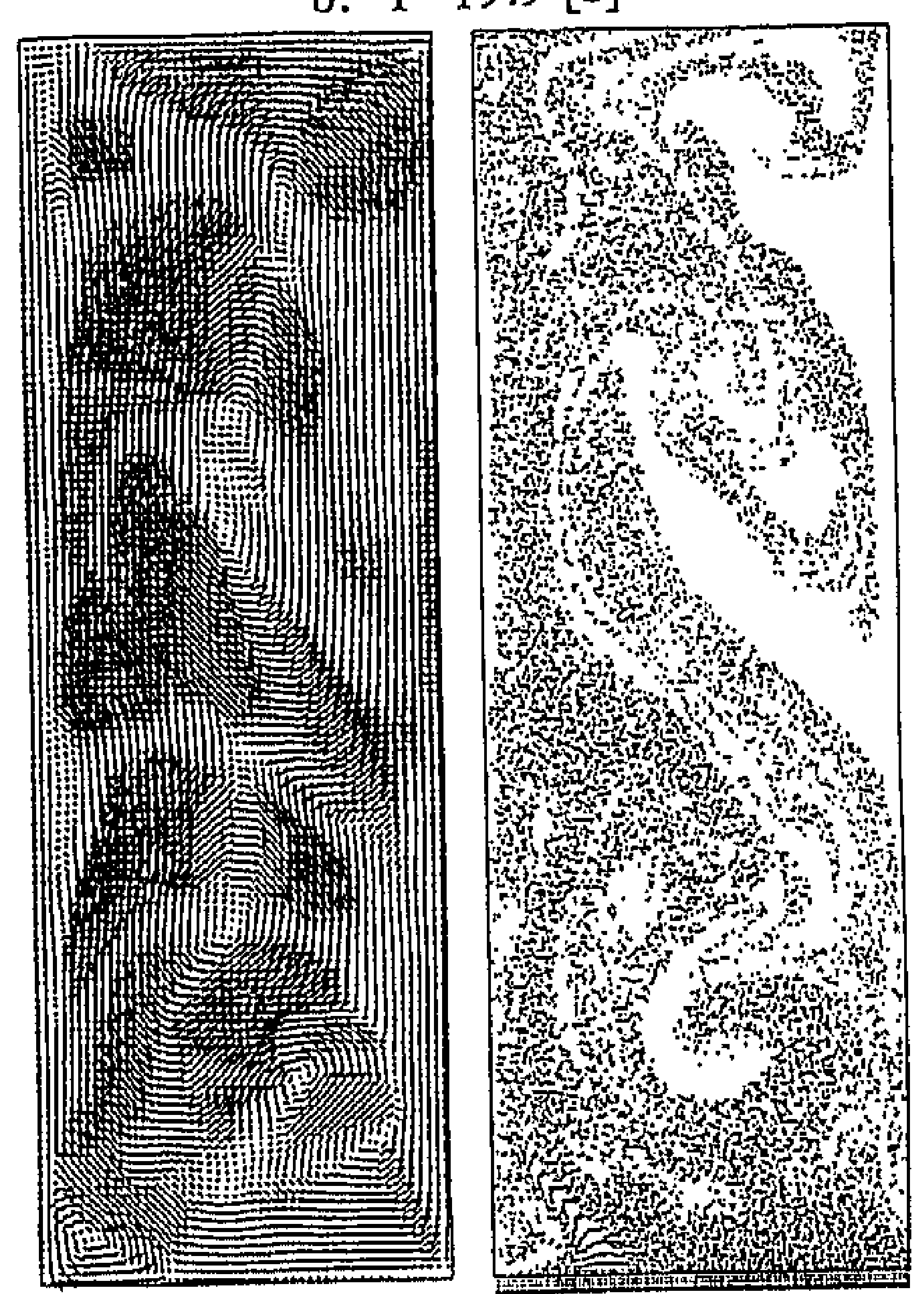

$\mathrm{d}: \mathrm{T}=110[\mathrm{~s}]$

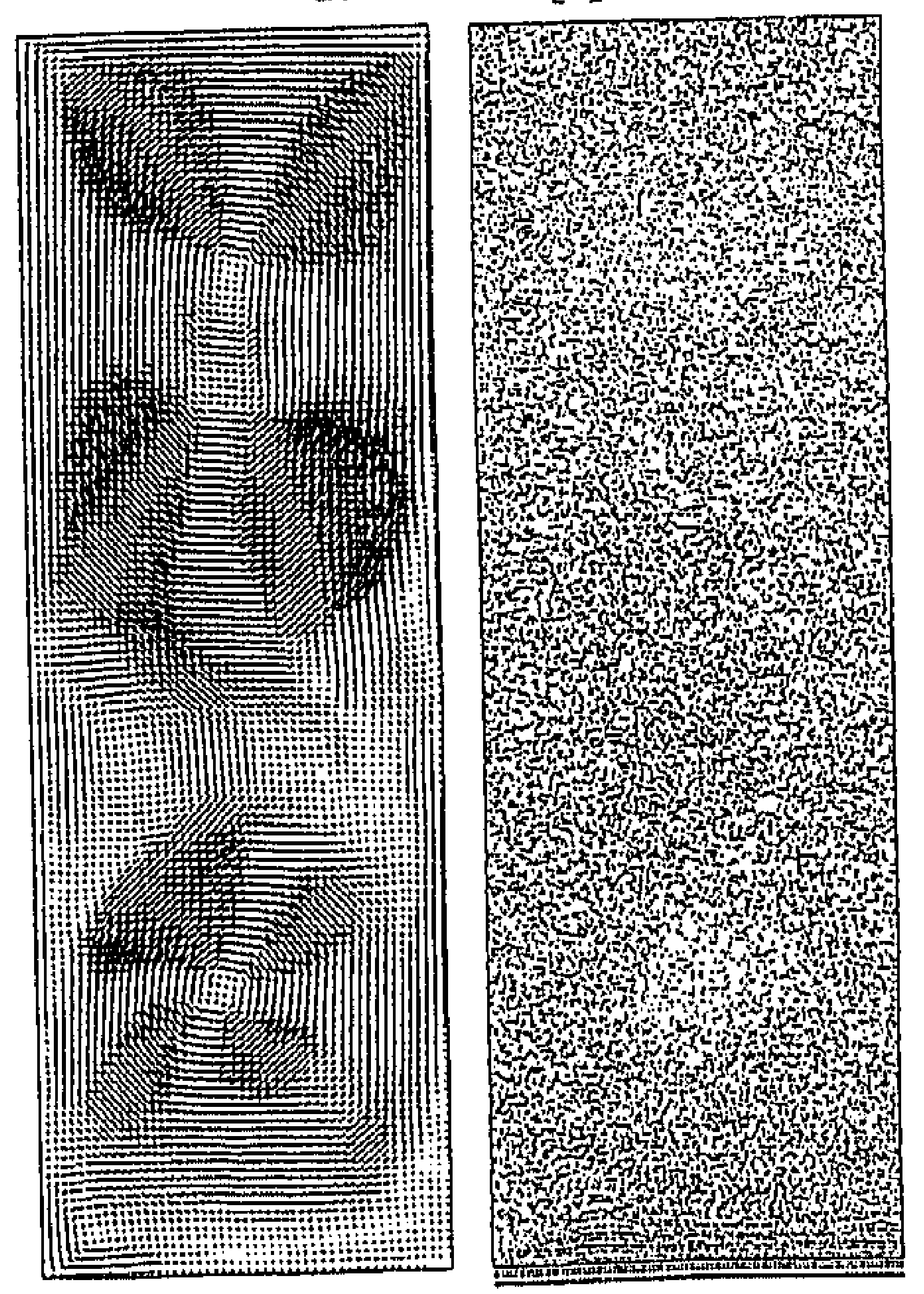

Fig. 11. Typical computational results obtained by Lapin and Lübbert (1994) with a mixed Eulerian-Lagrangian approach. Liquid phase velocity pattern (left) and the bubble positions (right) in a wafer column (diameter, $1.0 \mathrm{~m}$; height. $1.5 \mathrm{~m}$ ) where the bubbles are generated uniformly over in a wafer column (diameter, Lübbert, A., Numerical simulations of the dynamics of two-phase gas-liquid flows in bubble columns, p. 3661, copyright 1994 with permission from Elsevier Science.) 
SEPRAN package (Cuvelier et al., 1986). Recently Delnoij et al. (1997b) applied the VOF method to study the dynamics of single gas bubbles rising in a quiescent Newtonian liquid. They were able to demonstrate that the predicted bubble shape and the induced flow patterns in the liquid phase could be predicted very well as a function of the key physical properties of the liquid phase such as density, viscosity, and surface tension (see Section VI.D). The results reported by Tomiyama et al. are discussed in more detail below.

Tomiyama et al. (1993) applied the VOF method to analyze the motion of a single gas bubble rising in a liquid. They were able to show that the shape and terminal velocities of the gas bubbles could be predicted satisfactorily well over a wide range of Eotvös and Morton numbers. Figure 12a shows some typical results reported by Tomiyama et al. (1993) on the effect of the Morton number M on the shape and dynamics of a single bubble rising in a Newtonian liquid. For the purpose of reference in Fig. 12b the graphical correlation presented by Grace (1973) and Grace et al. (1976) is included, which takes into account the effect of fluid properties and equivalent bubble diameter $d_{b}$ on the bubble shape and the terminal velocity $V_{1}$ using three dimensionless quantities: the Reynolds number Re, the Morton number $M$, and the Eotvös number Eo defined by:

$$
\begin{aligned}
& \operatorname{Re}=\frac{\rho V_{1} d_{\mathrm{b}}}{\mu}, \\
& M=\frac{g \mu^{-4} \Delta \rho}{\rho^{2} \sigma^{3}}, \\
& E_{O}=\frac{g \Delta \rho d_{b}^{2}}{\sigma},
\end{aligned}
$$

where $\rho . \Delta \rho, \mu$, and $\sigma$ represent, respectively, the liquid density, the density difference between the liquid and gas, the dynamic shear viscosity of the liquid, and the surface tension of the liquid. From inspection of Figs. 12a and $b$ it can be concluded that the predicted effect of the Morton number at least qualitatively agrees. with those obtained from the graphical correlation presented by Grace (1973) and Grace et al. (1976).

Recently Lin et al. (1996) applied the VOF method to study the timedependent behavior of bubbly flows and compared their computational results with experimental data obtained with a particle image velocimetry (PIV) technique. In their study the VOF technique was applied to track several bubbles emanating from a small number of orifices. Lin $\mathrm{et}$ al. reported satisfactory agreement between theory and experiment.

b. Gas-Liquid Stirred Tank Reactors. CFD has also been applied to study the flow phenomena in gas-liquid stirred tank reactors. In many studies one-way cou- 

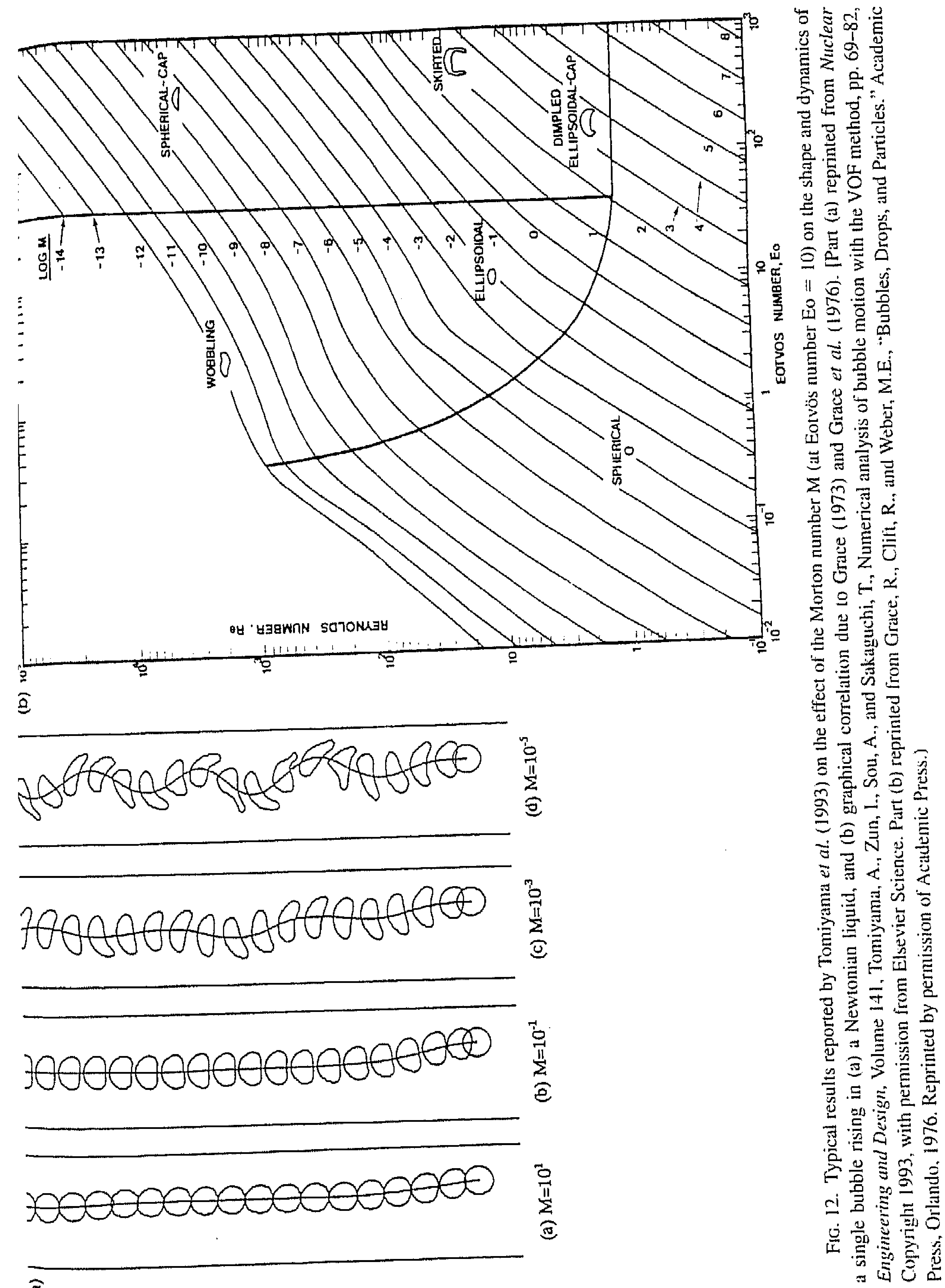
pling (Bakker and van den Akker, 1994) was assumed in the simulations although, in view of the reported values of the gas phase volume fraction $(5-10 \%)$, it would have been more appropriate to account for two-way coupling. This is to some extent due to the possibilities offered in (early versions) of commercial CFD packages. Another problem is the turbulence modeling, where due to the absence of more detailed knowledge often the standard single-phase $k-\epsilon$ model is used. Finally difficulties in accurately representing the impeller geometry are also encountered in this type of flow (also see Sections IV,A,1 and 2).

c. Other Gas-Liquid Reactors. In literature a variety of other CFD applications involving gas-liquid reactors have been reported such as hydrocyclones (Hargreaves and Silvester, 1990; Hsieh and Rajamani, 1991) and loop reactors (Sokolichin and Eigenberger, 1994).

d. Concluding Remarks. The studies reported in this section clearly show that for gas-liquid two-phase flow very promising results have been obtained in recent years. The results obtained with the mixed Eulerian-Lagrangian method and the VOF method in particular are very promising despite the fact that due to CPU constraints the analysis is limited respectively to moderate (typically $10^{5}$ ) and a relatively small (typically 10 ) number of bubbles.

Despite this significant progress, complicated problems still remain such as the prediction of the rate of bubble formation at gas distributors and the coalescence and breakup of bubbles in the gas-liquid dispersion. More general it is still impossible to predict flow regime transition in various types of gas-liquid twophase flow. Also the coupling to chemical transformation remains extremely difficult due to the fact that often an extremely fine spatial and temporal resolution has to be obtained especially in cases where chemically enhanced mass transfer takes place (Versteeg et al., 1989, 1990; Frank et al., 1995a,b). Nevertheless the coupling of mass transfer models described by Versteeg et al. and Frank et al. with mixed Eulerian-Lagrangian models as developed by Delnoij et al. (1997a) offers a potential to arrive at fundamental reactor models for bubble columns in the foreseeable future.

However, with respect to the mixed Eulerian-Lagrangian approach it should be mentioned that disagreement still exists on rather fundamental issues such as the correct description of the forces acting on a single bubble and the effect of other bubbles on these forces. In this area much fundamental work remains to be done. Moreover the extension of the mixed Eulerian-Lagrangian approach to the churn-turbulent or heterogeneous bubbly regime is of crucial importance due to the fact that this flow regime is frequently encountered in industrial applications involving bubble columns. In this respect a modeling approach is required that can simultaneously deal with "large" bubbles and "small" bubbles. In this respect 
a model that combines the features of the VOF method and the mixed Eulerian-Lagrangian method deserves attention. Last but not least the incorporation of turbulence effects is still problematic and should receive more attention in the future.

\section{Liquid-Liquid (L-L) Systems}

Liquid-liquid systems are encountered in many practical applications involving physical separations of which extraction processes performed in both sievetray and packed columns are well-known examples. In principle, all three methods discussed in Section III,B,2 can be used to model liquid-liquid two-phase flow problems. The added complexity in this case is the possible deformation of the interface and the occurrence of flow inside the droplet.

Of particular interest in this connection is the work of Ohta et al. (1995) who used a two-material VOF method to study the formation of a single droplet at an orifice in a pulsed sieve-plate column. They also compared the theoretically computed droplet sizes with those obtained from experiments using a high-speed video camera and reported good agreement between theory and experiment. In Fig. 13 the observed (a) and computed (b) droplet formation reported by Ohta et al. (1995) is shown. Note that the agreement between theory and experiment is reasonable although subtle differences between the computed and observed droplet shape can be clearly recognized. One of the great advantages of the twomaterial VOF method is its possibility to provide detailed information on the fluid flow both inside and outside the droplet. The VOF method is a very powerful numerical tool for analyzing complex free surface flows and its possible ability to study systems involving for example droplet interaction should be further explored. In this respect the required computational time may become problematic.

\section{Fluid-Solid (G-S and L-S) Systems}

Fluid-solid systems, especially in situations where the fluid is a gas. are very frequently encountered in various important industrial processes such as packedbed reactors, moving-bed reactors, fluidized-bed reactors, and entrained reactors.

a. Packed-Bed Reactors. Although flows in packed-bed reactors can also be classified as a chemically reactive single-phase flow in a complex geometry, the authors think that it is more appropriate to treat this type of flow as a special type of two-phase flow. The analysis of heat transfer processes and coupled chemical conversion in packed-bed reactors has traditionally received considerable attention from chemical engineers. In most of these studies rather simple representations of the prevailing flow patterns (i.e., plug flow) were used despite the fact that flow inhomogeneities are known to exist due to (local) porosity disturbances. 
(a)
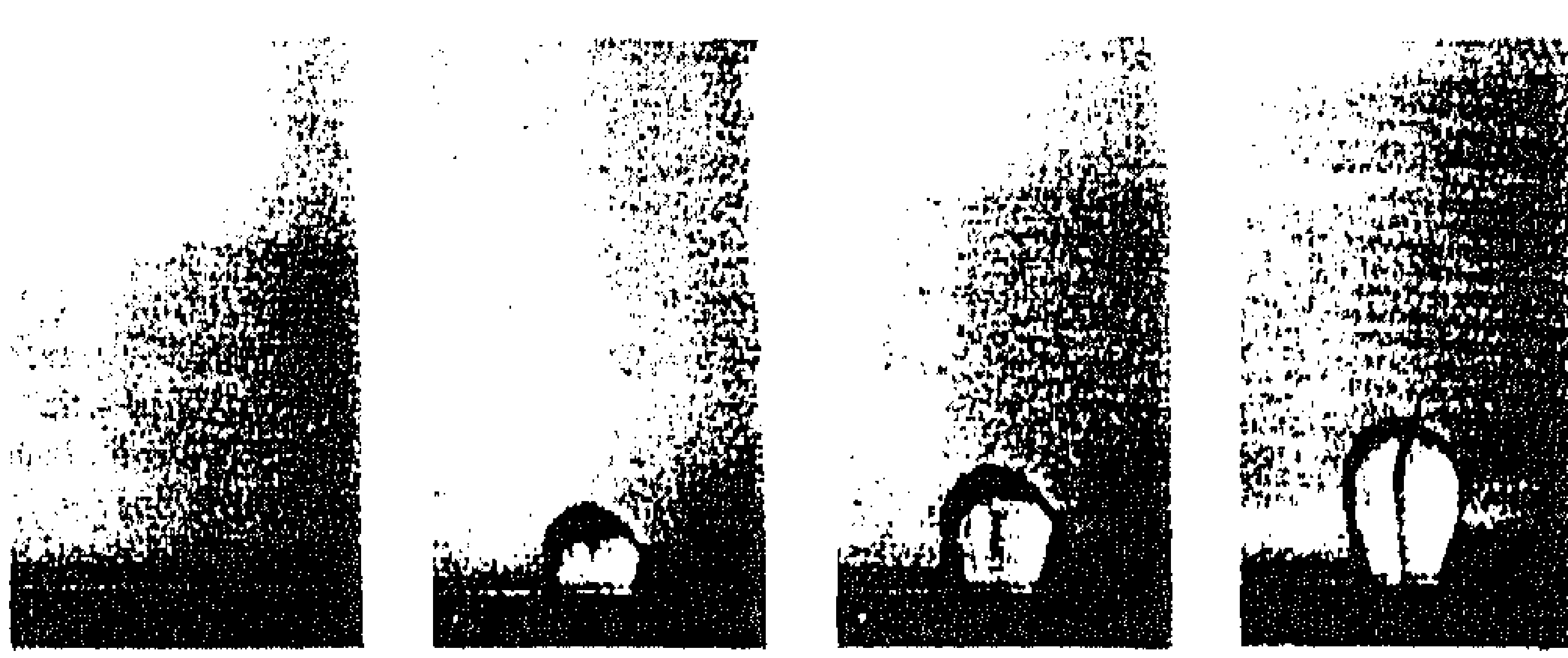

$\mathrm{T}=0.00[\mathrm{sec}]$

$\mathrm{T}=0.10[\mathrm{sec}]$
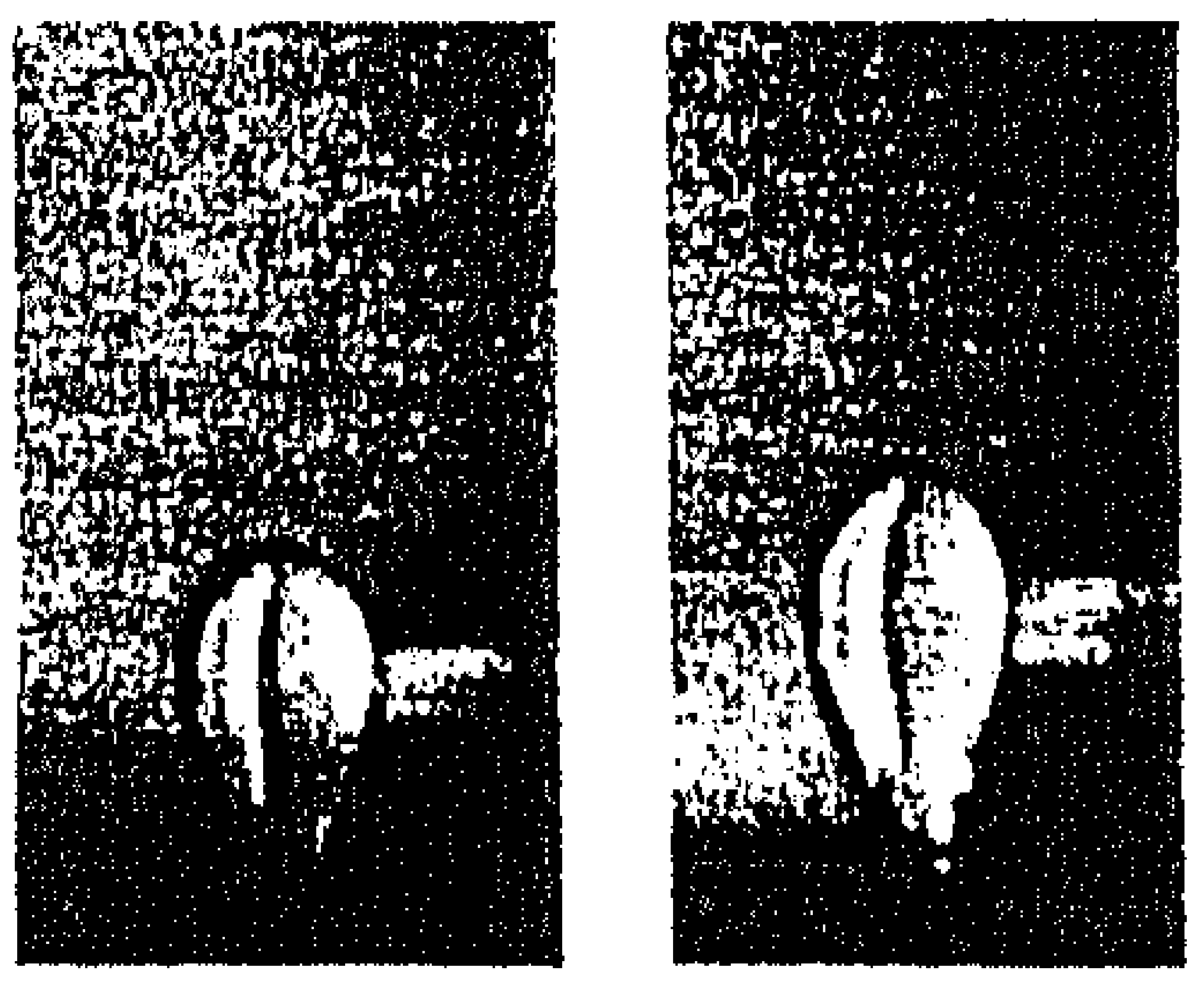

$\mathrm{T}=0.15[\mathrm{sec}]$

$T=0.20$ [sec]
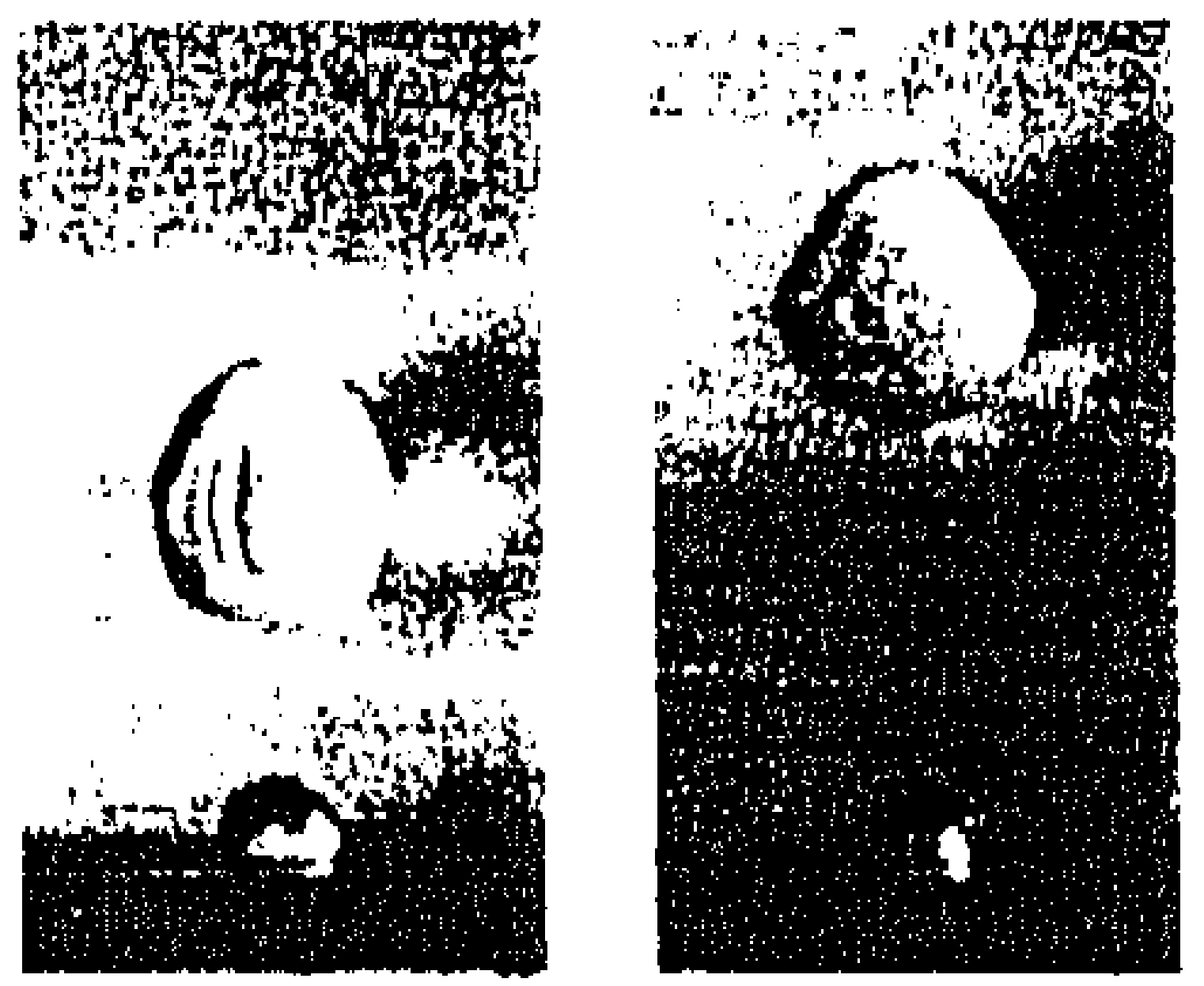

$\mathrm{T}=0.25[\mathrm{sec}]$

$\mathrm{T}=0.30[\mathrm{sec}]$

$T=0.35[\mathrm{sec}]$

$T=0.40[\mathrm{sec}]$

(b)

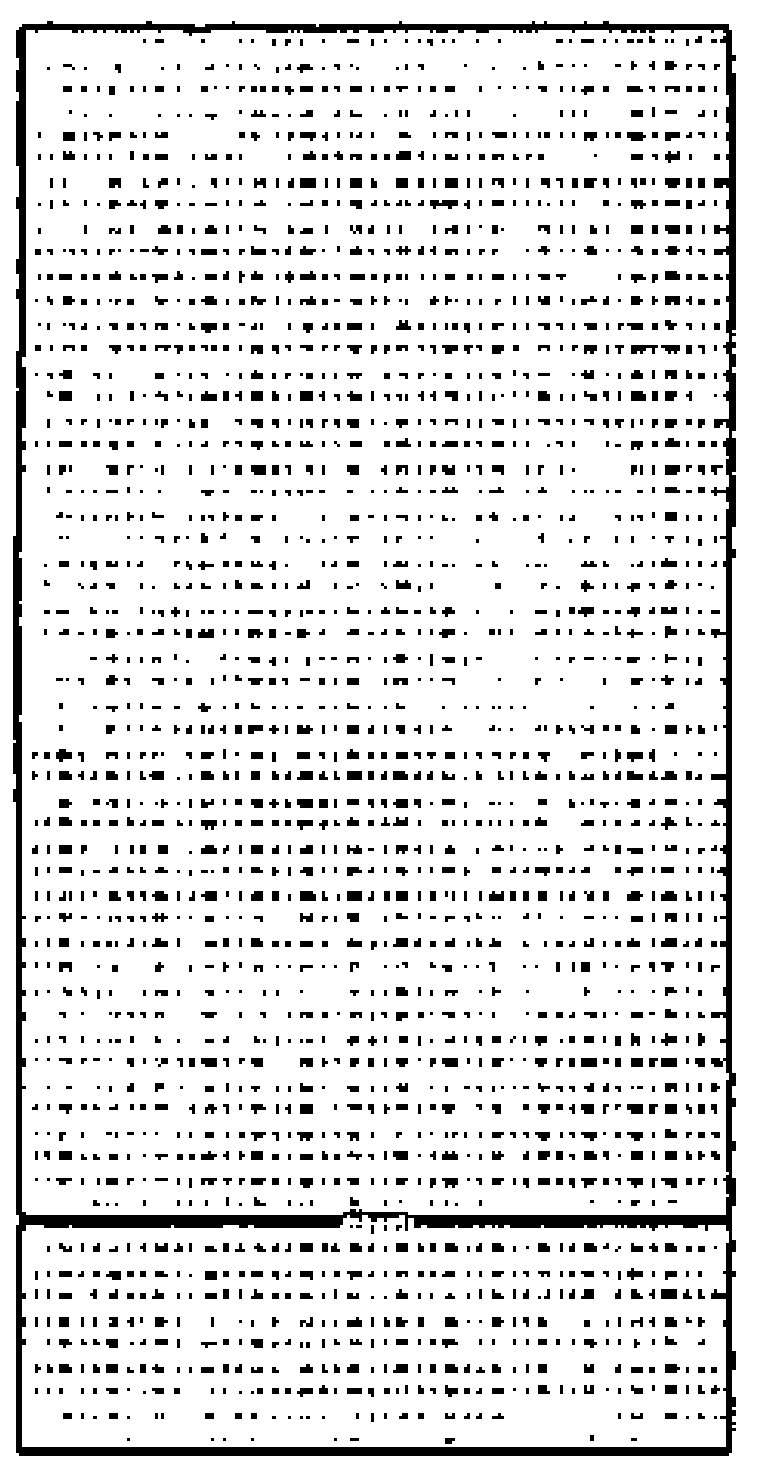

$\mathrm{T}=0.00[\mathrm{sec}]$

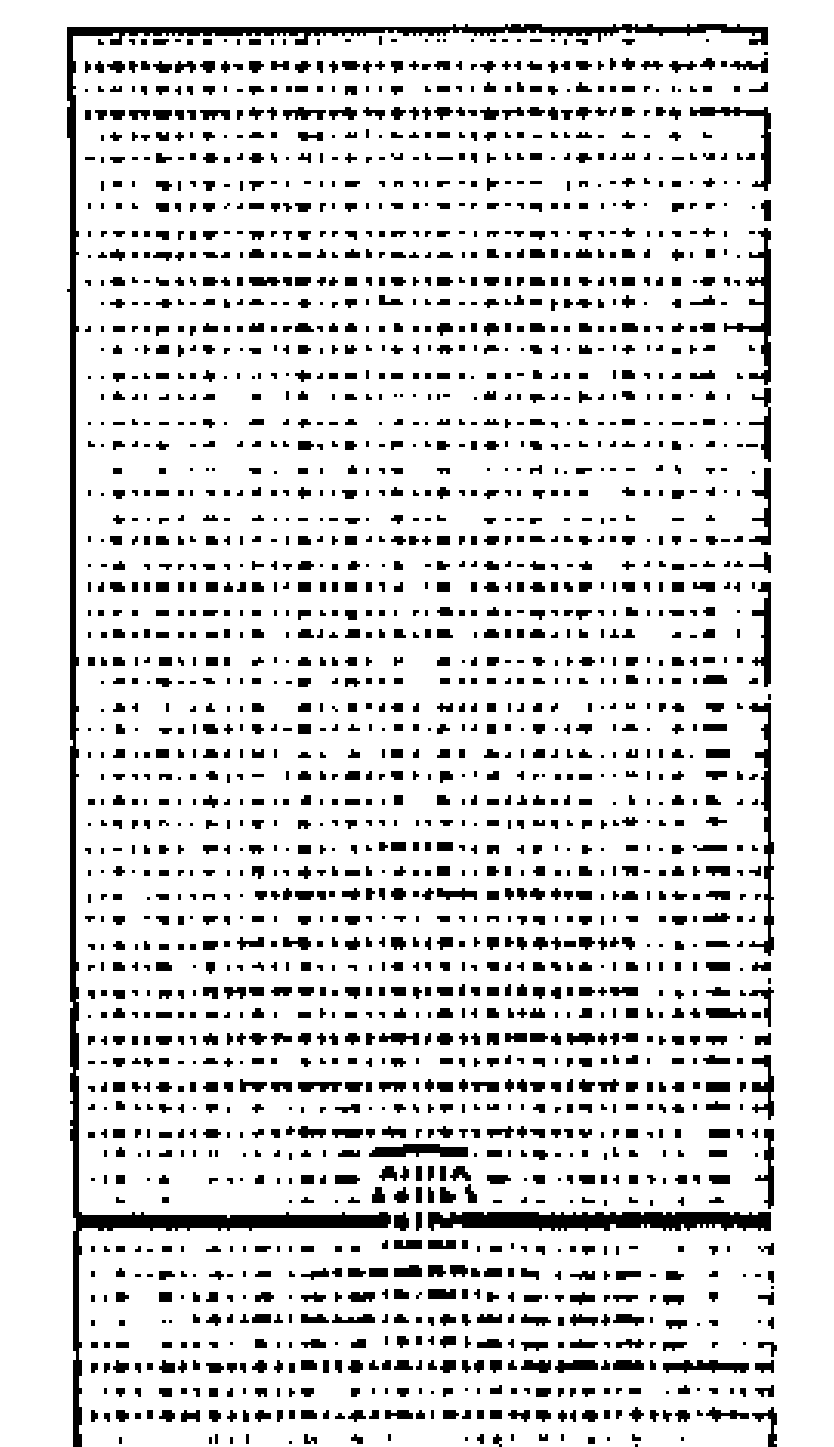

$\mathrm{T}=0.10[\mathrm{sec}]$

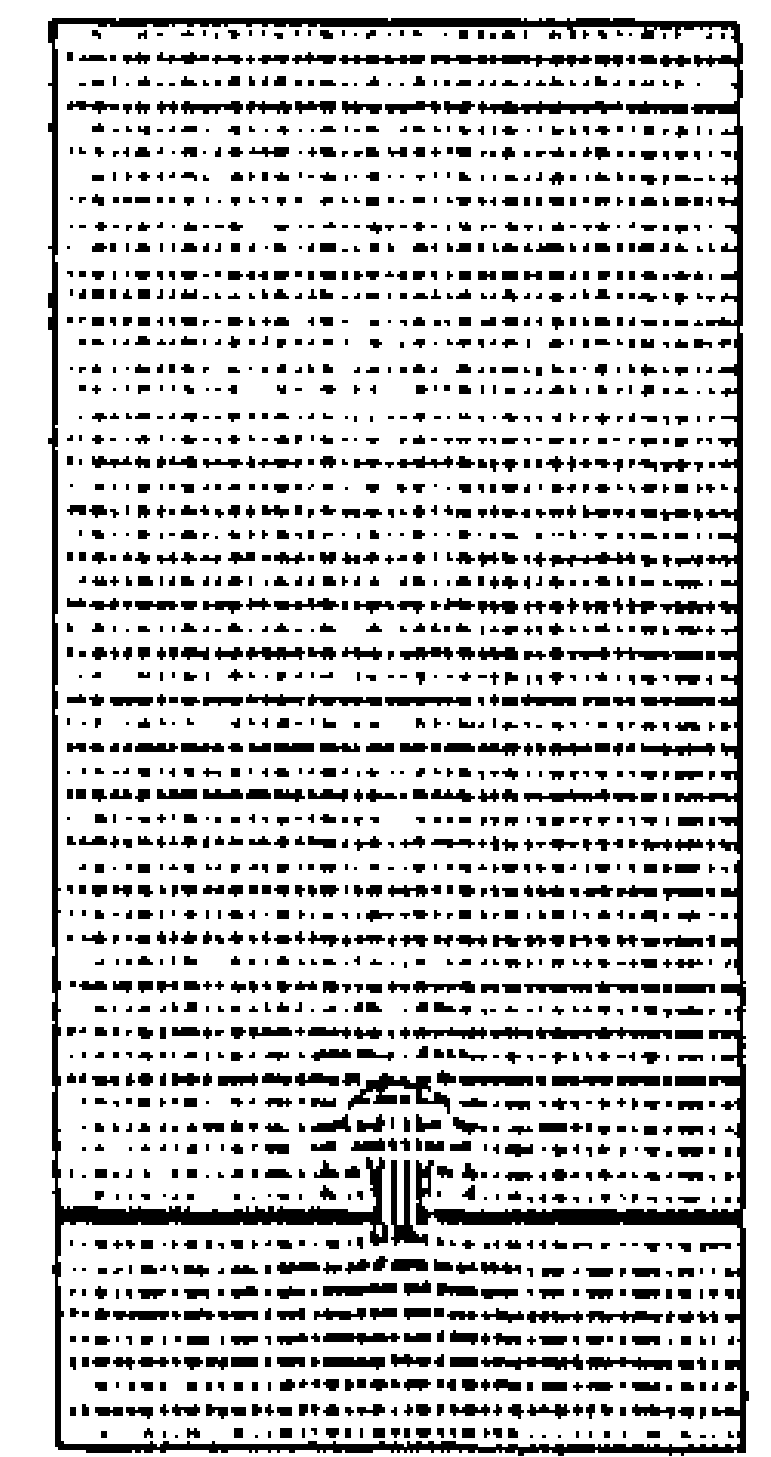

$\mathrm{T}=0.15[\mathrm{sec}]$

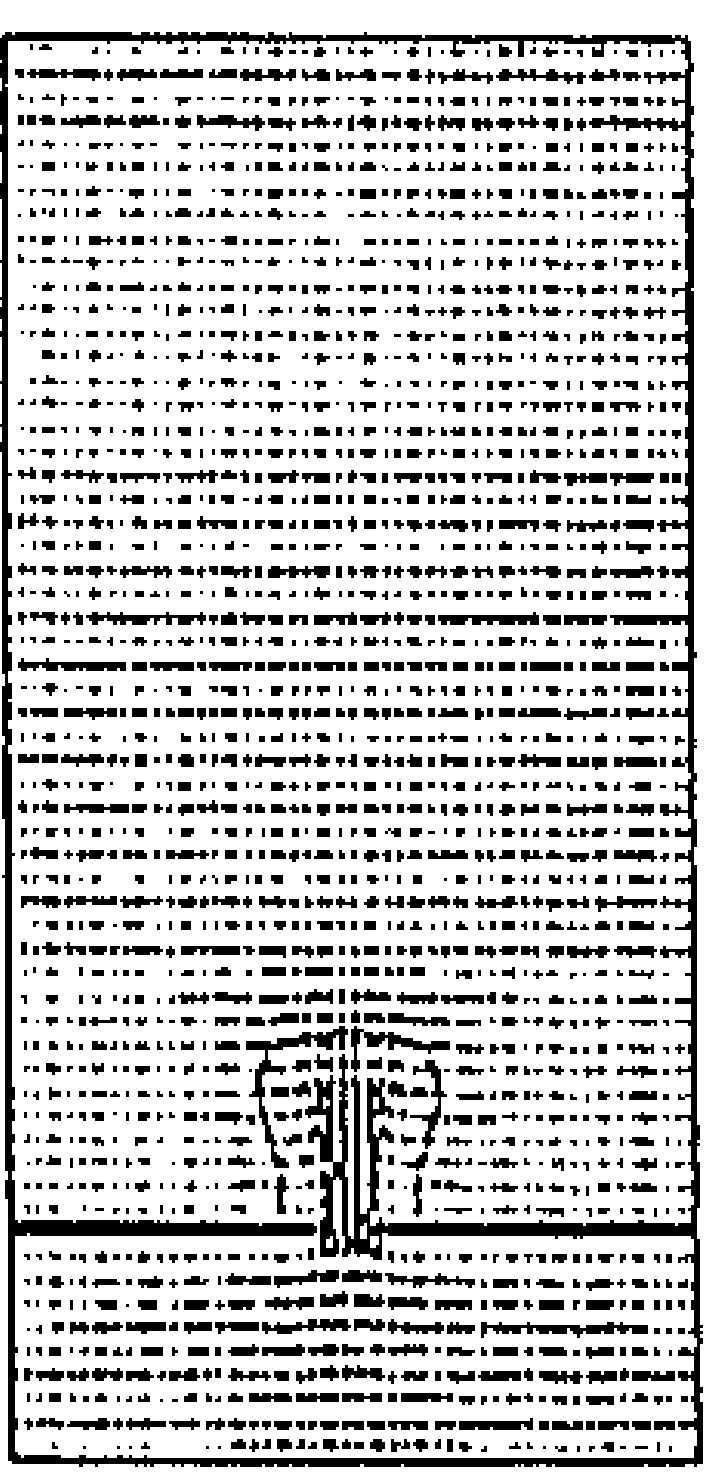

$\mathrm{T}=0.20[\mathrm{sec}]$

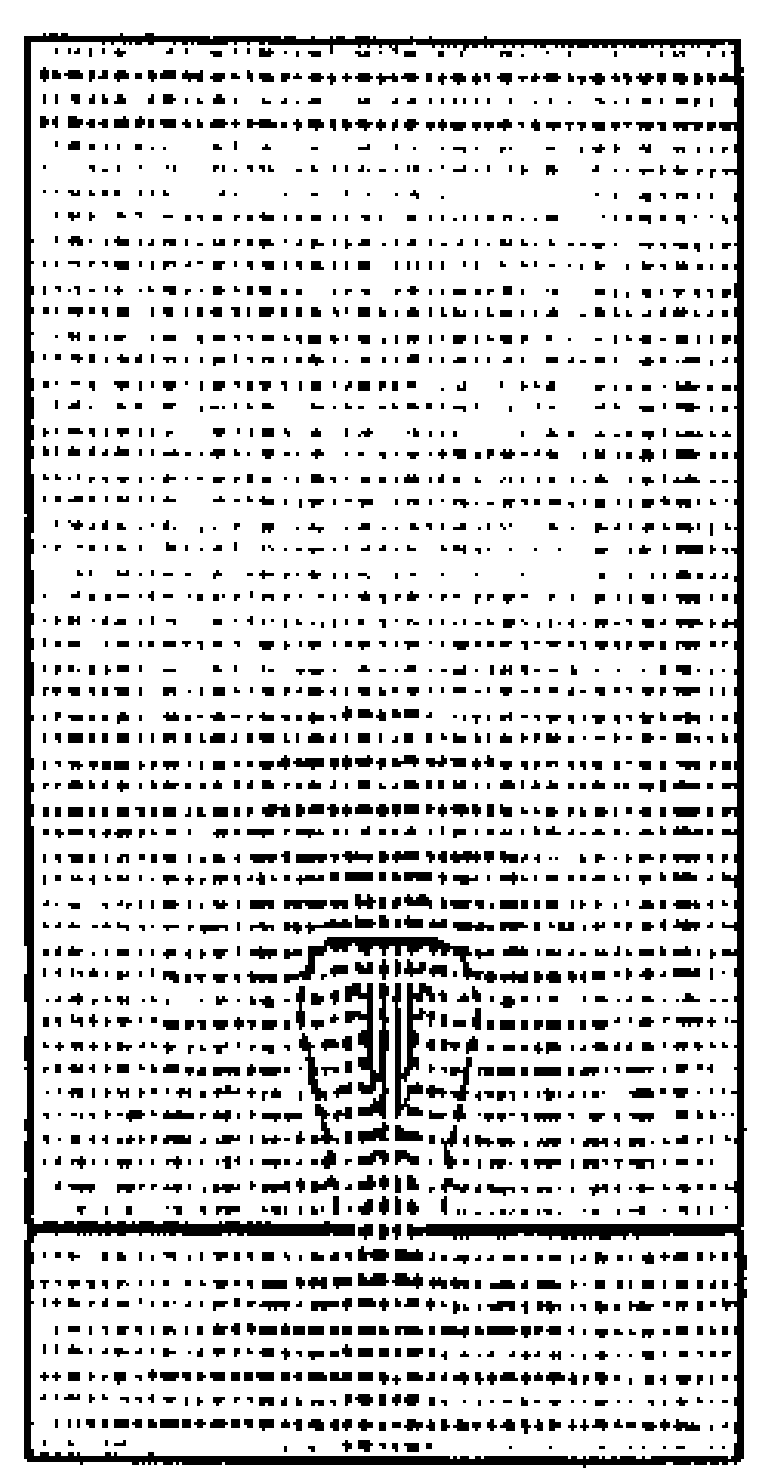

$\mathrm{T}=0.25[\mathrm{sec}]$

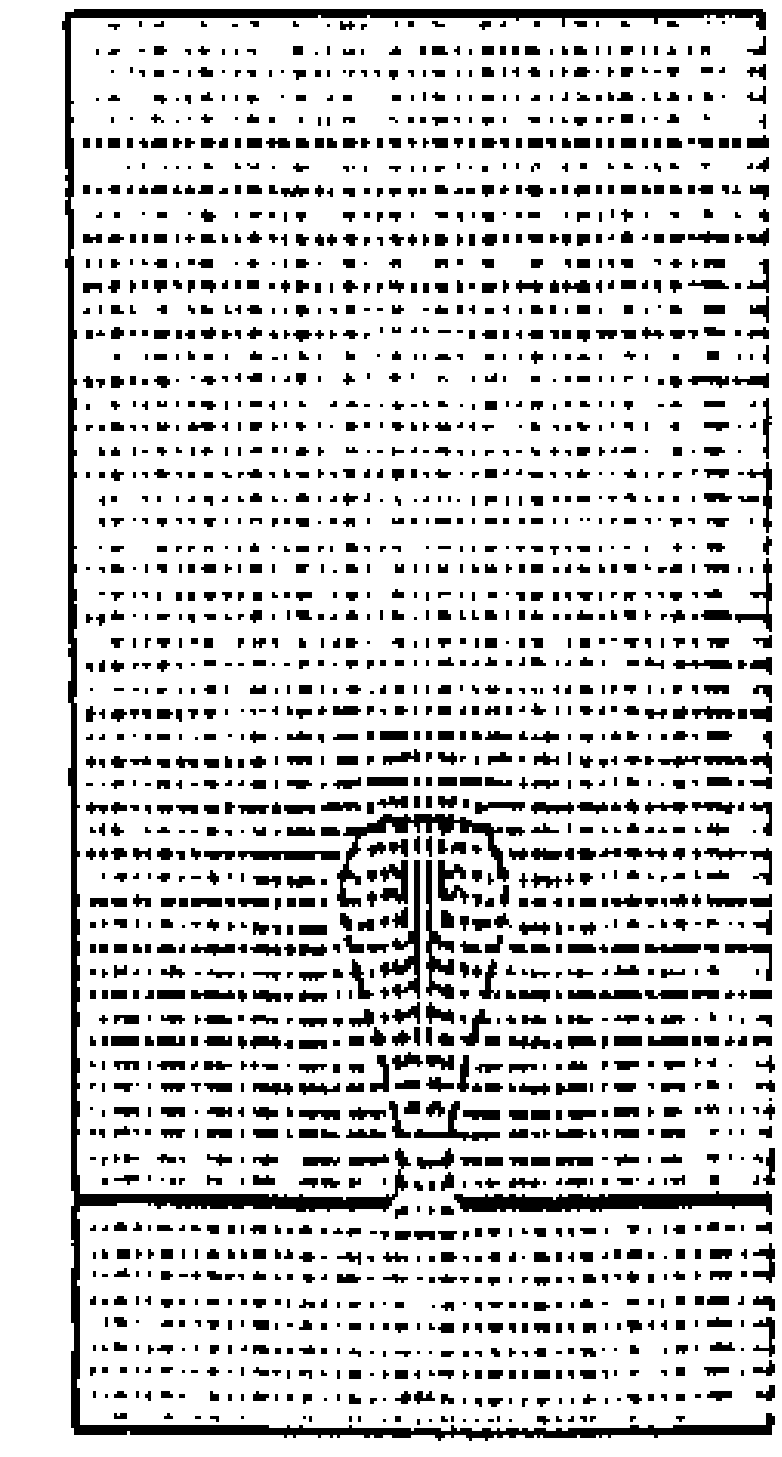

$T=0.30[\mathrm{sec}]$

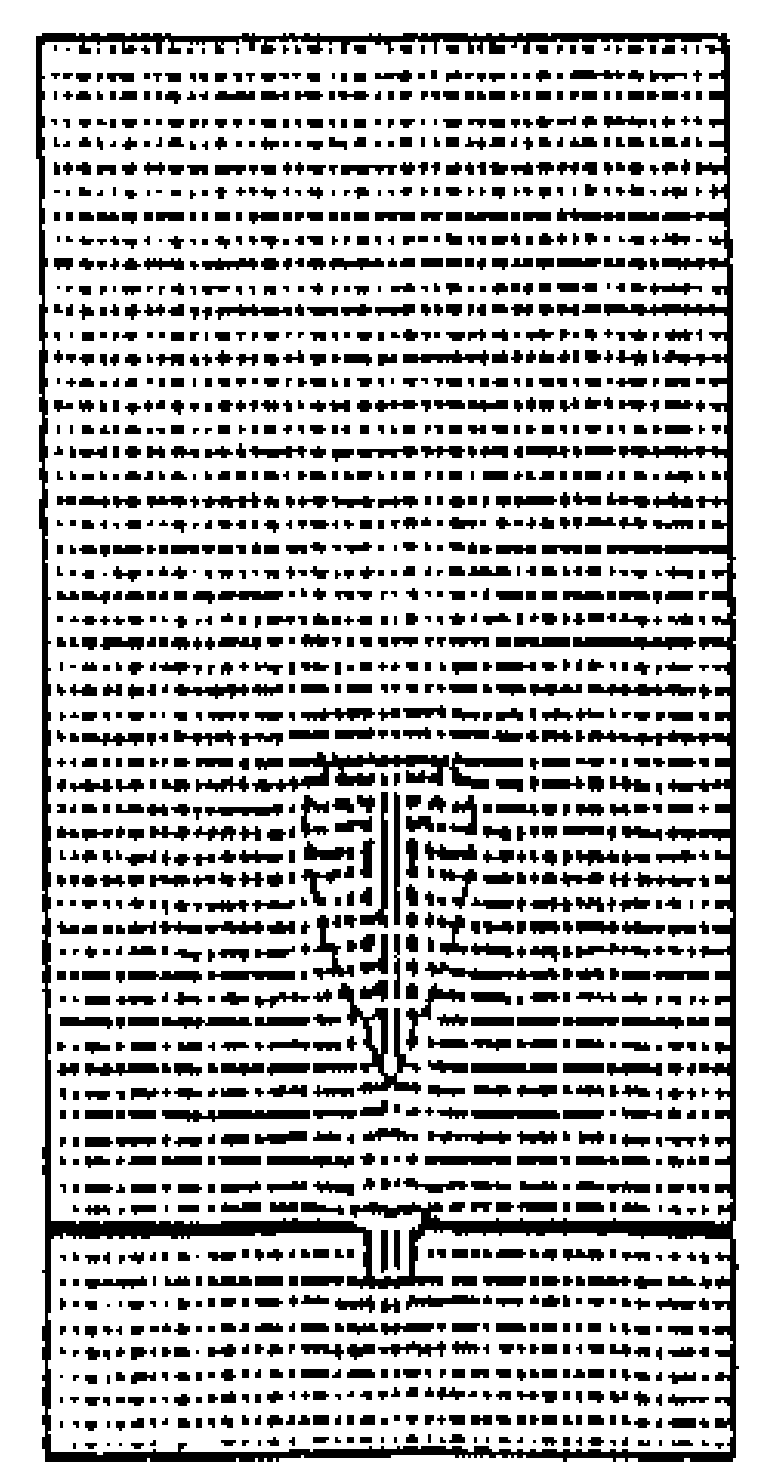

$\mathrm{T}=0.35[\mathrm{sec}]$

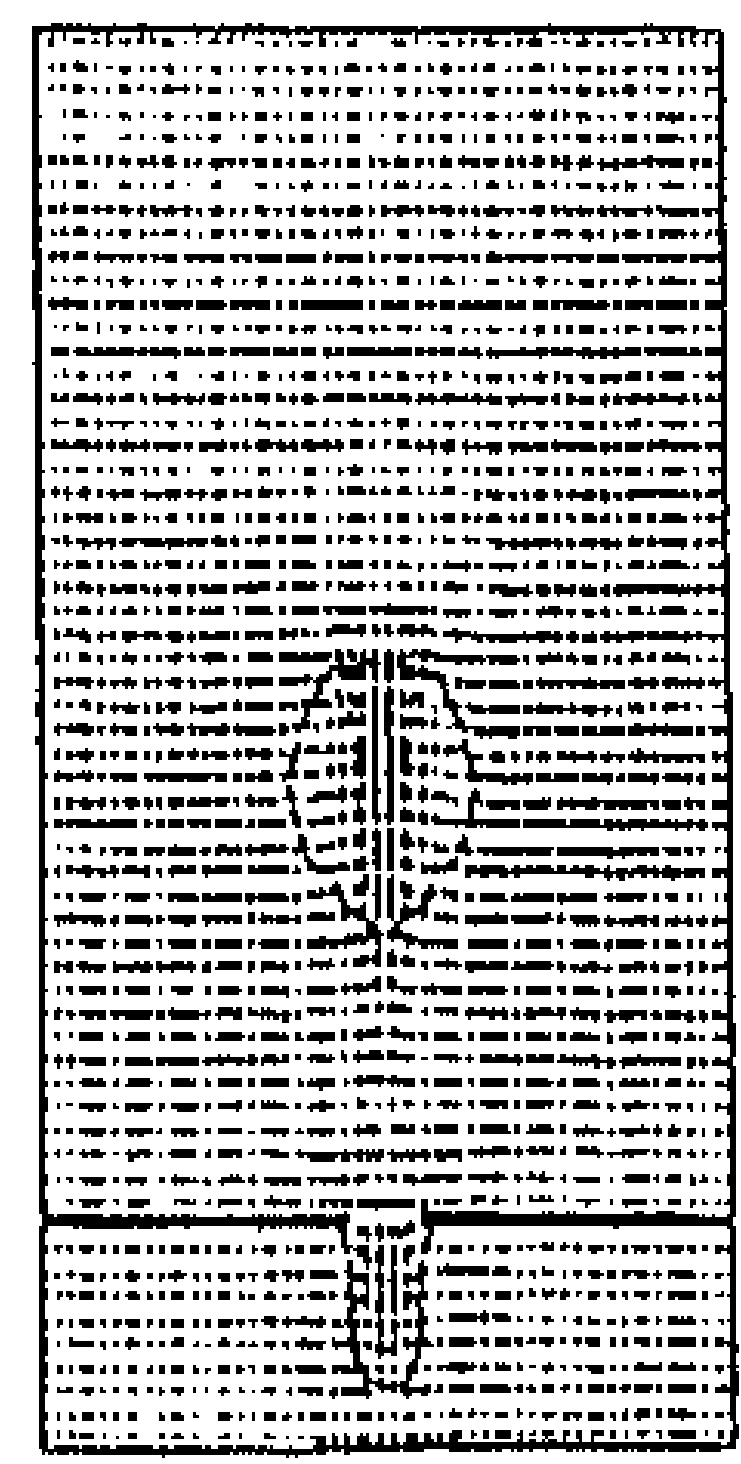

$\mathrm{T}=0.40[\mathrm{sec}]$

FIG. 13. (a) Observed and (b) computed formation of a droplet at an orifice in a pulsed sieveplate extraction column. (Reprinted from Chemical Engineering Science, Volume 50, Ohta M., el al.. Numerical analysis of a single drop formation process under pressure pulse condition, $\mathrm{pp}$. 2923-2931, copyright 1995, with permission from Elsevier Science.) 
A very important form of such disturbances is caused by the presence of the wall of the tube containing the packed bed. Vortmeyer and Schuster (1983) have used a variational approach to evaluate the steady two-dimensional velocity profiles for isothermal incompressible flow in rectangular and circular packed beds. They used the continuity equation, Brinkman's equation (1947), and a semiempirical expression for the radial porosity profile in the packed bed to compute these profiles. They were able to show that significant preferential wall flow occurs when the ratio of the channel diameter to the particle diameter becomes sufficiently small. Although their study was done for an idealized situation it has laid the foundation for more detailed studies. Here CFD has definitely contributed to the improvements of theoretical prediction of reactor performance.

Another possibility for modeling packed-bed reactors involves the use of a socalled unit cell approach where a suitable periodic structure in the packing is identified and subsequently used to define the boundaries of the computational domain. Due to the geometrical complexity the Huid flow (and other relevant equations have to be formulated and solved in curvilinear coordinates. In fact this approach has been followed for example, by Guj and De Matteis (1986) who used a MAC-like scheme (Welch et al., 1965) to solve the Navier-Stokes equations. For random packings the unit cell approach becomes much more difficult due to the fact that a suitable periodic structure is difficult to define.

b. Fluidized Bed Reactors. Another type of chemical reactor where the CFD approach has proven fruitful is the gas fluidized-bed reactor. These reactors find a widespread application in the petroleum, chemical, metallurgical, and energy industries (Kunii and Levenspiel, 1991) and significant research efforts have been made in both academic and industrial research laboratories to develop detailed micobalance models of gas-fluidized beds.

A very lucid review on fluidized-bed modeling and analysis of existing hydrodynamic models in terms of Occam's razor (a philosophical maxim based on the following principle: "It is futile to do with more what can be done with fewer") has been given by Clift (1993). Jackson (1994) has recently prepared a review on the state of the art of our understanding of the mechanics of fluidized beds and emphasizes that despite the fact that the goal of deriving exactly correct equations on the basis of the local instant formulation may be unattainable in the future, for many practical purposes it is acceptable to use a set of equations that is good enough (i.e., with acceptable engineering accuracy) to describe the behavior of a range of situations relevant to the chemical engineer.

Pritchett et.al. (1978) were the first to report numerical solutions of the nonlinear equations of change for fluidized suspensions. With their computer code, for the first time, bubbles issuing from a jet with continuous gas through-flow, could be calculated theoretically. Figure 14 shows as an illustration the computed mo- 

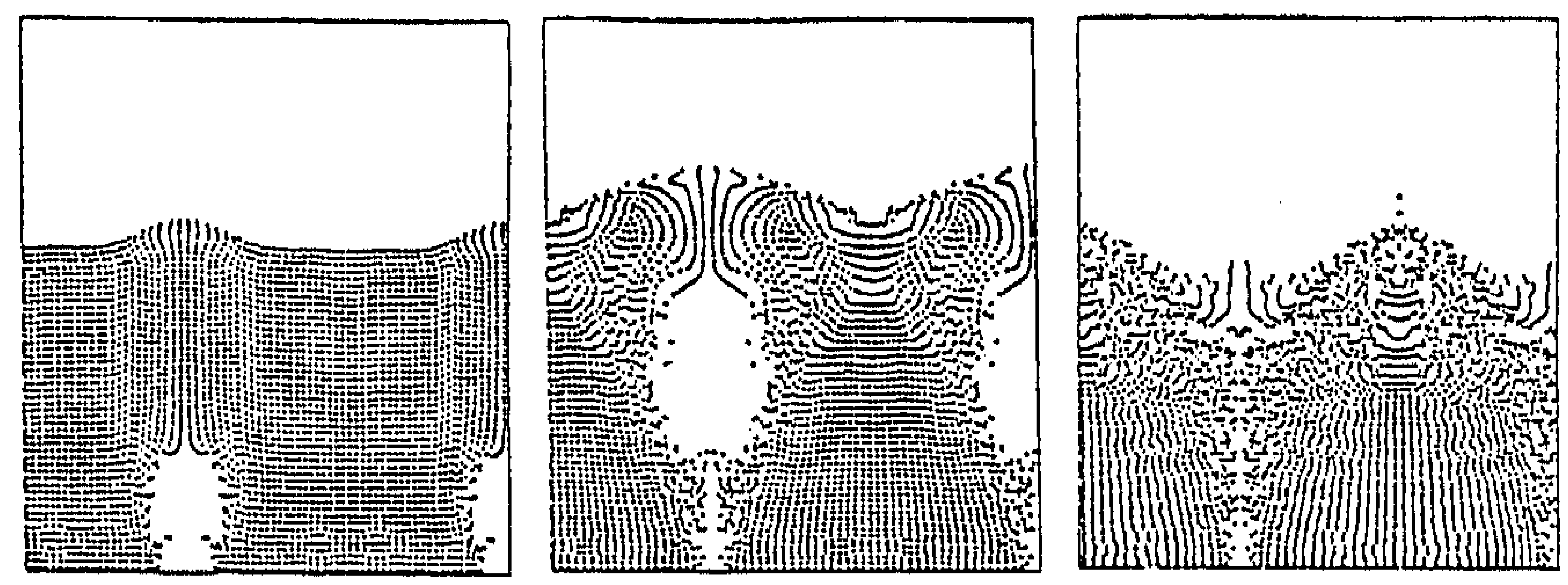

FIG. 14. Computed motion of a collection of pseudo-Lagrangian particles due to the evolution and propagation of gas bubbles in a section of a hypothetical two-dimensional gas fluidized bed [From Pritchett, J.W., Blake, T.R., and Garg, S.K. A numerical model of gas fluidized beds. AIChE Symp. Ser: 176, 74, 134 (1978). Reproduced with permission of the American Institute of Chemical Engineers. Copyright $(\mathcal{O} 1978$ AIChE. All rights reserved.]

tion of a collection of pseduo-Lagrangian particles due to the evolution and propagation of bubbles in a section of a hypothetical two-dimensional gas fluidized bed. Schneyer et al. (1981) extended this hydrodynamic model to a complete reactor model for a fluidized-bed coal gasifier. Their model incorporates the kinetics of the heterogeneous and homogeneous chemistry of combustion and gasification and probably represents the most ambitious and comprehensive fluidization modeling effort to date. A more or less parallel development has been made by the Jaycor group (Henline et al., 1981; Scharff et al., 1982) who also made an attempt to model a fluidized bed coal gasifier from first principles.

In the literature a relatively large number of publications exist which deal with the CFD analysis of gas-fluidized beds using two-fluid models (Gidaspow and Ettehadieh, 1983; Ettehadieh et al., 1984; Syamlal and Gidaspow, 1985; Gidaspow, 1986; Bouillard et al., 1989; Kuipers 1990; Kuipers et al., 1991, 1992a,b.c. 1993; Nieuwland et al. 1996a). These studies have clearly revealed that many important key properties of bubbling fluidized beds, that is, gas bubble behavior. spatial voidage distributions, and wall-to-bed heat transfer processes can be predicted satisfactorily from two-fluid simulations (see Section VI,A). However, the agreement between theory and experiments is not perfect and has been attributed among other factors to the simple solids rheology (i.e., Newtonian behaviour) incorporated in the two-fluid models. Further theoretical developments have led to the application of the kinetic theory of granular flow (KTGF) to gas-fluidized beds (Ding and Gidaspow, 1990; Manger, 1996) of which its ability to provide a priori information on the viscosities of the particulate phase can be mentioned as an important advantage over the constant-solids-viscosity two-fluid models. It has however not yet been demonstrated that the KTGF based model predicts more realistic gas bubble behavior in comparison with the constant-solids-viscosity two-fluid models.

CFD has also been applied to predict the flow patterns in the circulating fluidized bed (CFB) riser (Tsuo and Gidaspow, 1990). Despite their widespread in- 
dustrial application in. for example, petrochemical and energy production processes, our understanding of the hydrodynamics of these systems is, unfortunately, still very limited. For reliable design of large-scale CFB risers the designer requires accurate information regarding the spatial solids distribution, which is generally inhomogeneous in both axial and radial direction. The inhomogeneous solids distribution determines the gas-solid phase flow patterns to a large extent and thereby significantly influences riser reactor performance. Understanding and a priori prediction of this and related hydrodynamic aspects of riser flow are of crucial importance for design purposes. In literature lateral solids segregation has been attributed to interaction between gas phase eddies and dispersed particles and additionally to direct collisional interaction between particles. In most studies the direct particle-particle collisions have been described using KTGF, which is basically an extension of the Chapman-Enskog theory of dense gases. The KTGF was first applied by Sinclair and Jackson (1989) to predict successfully lateral segregation in CFB risers. Pita and Sundaresan (1991, 1993) further developed this model and found strong parametric sensitivity of their model predictions with respect to the value of the restitution coefficient $e$ for particle-particle collisions. In cases where realistic $e$ values were used, no lateral segregation could be predicted. In none of these models was turbulence accounted for, and very limited comparison with experimental data was reported in these studies.

Theologos and Markatos (1992) used the PHOENICS program to model the flow and heat transfer in fluidized catalytic cracking (FCC) riser-type reactors. They did not account for collisional particle-particle and particle-wall interactions and therefore it seems unlikely that this type of simulation will produce the correct flow structure in the riser reactor. Nevertheless it is one of the first attempts to integrate multiphase hydrodynamics and heat transfer.

A more recent and very promising development in the modeling of (dense) gas-particle flows, as encountered in bubbling fluidized beds and circulating fluidized beds, is the discrete particle approach (Tsuji et al., 1993; Kawaguchi et al., 1995; Tanaka et al., 1996; Hoomans et al., 1996) where the motion of individual spherical particles is directly calculated from the forces acting on them, accounting for the particle-particle and particle--wall interactions and drag between the particles and the interstitial gas phase. Tsuji et al. used the distinct element model (DEM), originally developed by Cundall and Strack (1979), to represent the interaction forces resulting from particle-particle and particle-wall encounters. The model developed by Tsuji et al. in fact represents a so-called "soft sphere" model because the particles are thought to undergo deformation during their contact where the interaction forces in both the normal and tangential direction are calculated from simple mechanical representations involving a spring, a dashpot, and a slider. Usually this model type requires the spring constant, the damping coefficient of the dashpot, and the friction coefficient of the slider as input parameters. 
The model developed by Hoomans et al. (1997) represents a so-called "hard sphere" model because in their model quasi-rigid spheres are implied which undergo a quasi-instantaneous collision where point contact prevails. Their model requires specification of two phenomenological parameters, namely, the coefficient of restitution and the friction coefficient. Some illustrative results that have been obtained from this type of modeling of gas fluidized beds are presented in Section VI.B. Hoomans et al. (1998) have compared the predictions obtained from hard sphere models with those of soft sphere models and found that the macroscopic system behavior predicted by both types of models is very similar provided that the spring constant used in the DEM model is given a sufficiently large value.

In the discrete particle models discussed so far the hydrodynamic interaction between particles was neglected. For gas-solid systems this assumption will be valid but for liquid-solid systems this type of interaction will become important and has to be accounted for. Stokesian dynamics (Brady and Bossis, 1988) constitutes a very powerful tool and offers significant possibilities for understanding of suspension dynamics on a more fundamental basis. For example, Ichiki and Hayakawa (1995) presented a Stokesian dynamics based model in which hydrodynamically interacting granular particles were considered. They neglected the inertial terms in the Navier-Stokes equations describing the interstitial fluid flow and therefore their model is only valid for relatively small particle Reynolds numbers (i.e., $\operatorname{Re}_{p}<1$ ). These computations are very CPU intensive and at present only simulations with a relatively low number of particles are possible. Despite these (serious) limitations this type of work is very inportant because it can lead to improved constitutive equations for fluid-particle interactions, which can be used subsequently in both discrete particle models which do not treat the flow of the continuous phase at the scale of a single particle.

Koelman and Hoogerbrugge (1993) have developed a particle-based method that combines features from molecular dynamics (MD) and lattice-gas automata (LGA) to simulate the dynamics of hard sphere suspensions. A similar approach has been followed by $\mathrm{Ge}$ and $\mathrm{Li}$ (1996) who used a pseudo-particle approach to study the hydrodynamics of gas-solid two-phase flow. In both studies, instead of the Navier-Stokes equations, fictitious gas particles were used to represent and model the flow behavior of the interstial fluid while collisional particle-particle interactions were also accounted for. The power of these approaches is given by the fact that both particle-particle interactions (i.e., collisions) and hydrodynamic interactions in the particle assembly are taken into account. Moreover, these modeling approaches do not require the specification of closure laws for the interphase momentum transfer between the particles and the interstitial fluid. Although these types of models cannot yet be applied to macroscopic systems of interest to the chemical engineer they can provide detailed information which can subsequently be used in (continuum) models which are suited for simulation of macroscopic systems. In this context improved rheological models and boundary condition descriptions can be mentioned as examples. 
c. Concluding Remarks. On the basis of the aforementioned results it is clear that significant progress has been made in modeling gas-particle flows and to a lesser extent liquid-particle flows. There remain however very significant challenges of which we would like to mention the following:

- Detailed modeling of the (mutual) interaction between turbulent flowing fluids and suspended particles

- Accurate description of particle-particle and particle-wall interactions including effects due to surface roughness and deviations from spherical particle shape

- Development of improved closure laws for the rheological description of fluidized suspensions on basis of discrete particle models

- Prediction of regime transition in gas-particle flows

- Incorporation of chemical conversion models in multiphase flow simulation

\section{Gas-Liquid-Solid Systems}

In many commercial processes efficient transfer of soluble substances or heat between a gas and a (reactive) liquid is achieved by contacting the phases in the presence of a packing where the function of the packing is the creation of sufficient contact area between the gas and liquid phase. In addition the packing can also act as a catalyst to enhance desired chemical transformations. Although the flow of the gas-liquid mixture through the packing can be classified as a two-phase flow in a complex geometry the authors think that it is more appropriate to treat this type of flow as a special type of three-phase flow. Such three-phase flows are encountered for example in trickle-bed reactors. In addition to these reactors other three-phase reactors find frequent application in commercial processes such as slurry reactors and three-phase fluidized-bed reactors. Contrary to the situation for trickle-bed reactors, in this case the solid phase (usually a catalyst) is suspended in the (continuous) liquid phase and can move freely in the reactor. The state of the art of CFD analysis of these three-phase reactors is briefly discussed.

a. Trickle-Bed Reactors. Unfortunately, very little progress has been made up to now on the CFD analysis of trickle-bed reactors. The reason for this state of the art is due to (1) the great geometrical complexity of the packing (often a catalyst), (2) the fact that we have to deal with a complex free surface flow with a very complex interfacial structure (i.e., partially wetted packing) where in certain flow regimes the liquid film interacts with the gas phase, and (3) the prevailing flow regime in a trickle-bed reactor can depend on the flow history experienced by the packing. Due to the aforementioned difficulties it will be very difficult to obtain significant progress in CFD modeling of trickle-bed reactors in the near future. and as a first step toward a more fundamental understanding of these complex systems approaches as followed by Melli and Scriven (1991) will probably be more fruitful. 
b. Slurry Reactors. As far as the computation of macroscopic flow patterns is concerned, the CFD analysis of slurry reactors shows some progress but unfortunately very little work has been done yet in this area, and in the few existing studies no comparison with experimental data is reported. For example, very recently Hamill et al. (1995) applied the CFDS-FLOW3D package to study the three-phase flow of solids, liquid, and a gas in a mixing vessel agitated by five impellers. They used the standard $k-\epsilon$ turbulence model and an explicit model for the modeling of the fluid flow around the impellers. Although their computational results appear reasonable, no comparison with experimental data was made in this case. Torvik and Svendsen (1990) used a multifluid formulation to model slurry reactors.

Despite the fact that there is some progress in modeling the macroscopic flow structure of slurry reactors, a number of microscopic phenomena are very difficult to capture in macroscopic flow simulation models such as the possible accumulation of solid particles near the gas-liquid interface, which significantly affects the mass transfer characteristics of the slurry system (Beenackers and van Swaaij, 1993).

c. Three-Phase Fluidized Beds. Similar to the situation for slurry reactors very little progress is seen in this area, which seems understandable in view of the remaining difficulties in modeling gas-liquid and liquid-solid two-phase flow.

\section{State of the Art of CFD in Chemical Reaction EngineERing}

As is evident from the examples mentioned in the preceding sections it has hopefully become apparent that in various areas of single-phase and multiphase flows significant progress has been made in CFD-based system descriptions. In

TABLE II

Present Situation and Prospects for CFD in CRE Involving Single-Phase Flon'

\begin{tabular}{|c|c|c|}
\hline Flow Category & Progress & Future Trends and Areas of Interest \\
\hline Laminar flows & Significant & $\begin{array}{l}\text { More complex geometries + interaction with mixing } \\
\text { and chemical transformation processes }\end{array}$ \\
\hline Turbulent flows & Reasonable & $\begin{array}{l}\text { Development of accurate and reliable turbulence mod- } \\
\text { els for enginering applications + use of LES and } \\
\text { DNS for generation of databases for turbulence } \\
\text { quantities }\end{array}$ \\
\hline Non-Newtonian flows & Reasonable & $\begin{array}{l}\text { More complex materials and more complex geometries } \\
+ \text { improved numerical solution procedures }+ \text { exten- } \\
\text { sion to multiphase flow }\end{array}$ \\
\hline Chemically reactive flows & Reasonable & $\begin{array}{l}\text { Application to more complex reaction schemes and } \\
\text { more complex geometries }+ \text { extension to multiphase } \\
\text { flow }\end{array}$ \\
\hline
\end{tabular}


Tables II and III, respectively, the progress and the future trends and remaining difficulties in systems involving single-phase flows and multiphase flows are briefly summarized.

TABLE III

Present Situation and Prospects for CFD in CRE Involving Multiphase Flow

\begin{tabular}{|c|c|c|}
\hline Flow Category & Progress & Future Trends or Problem Areas \\
\hline Gas--liquid & Reasonable & $\begin{array}{l}\text { Turbulence modeling + modeling of interfa- } \\
\text { cial transport phenomena + prediction of } \\
\text { flow regime transition + interaction of hy- } \\
\text { drodynamics with chemical transformation } \\
\text { processes }\end{array}$ \\
\hline Bubble columns & Reasonable & Modeling of churn-turbulent flow regime \\
\hline Loop reactors & Reasonable & Modeling of churn-turbulent flow regime \\
\hline Stirred tanks & Realsonable & $\begin{array}{l}\text { Improved geometrical representation of im- } \\
\text { peller and balffes }\end{array}$ \\
\hline Hydrocyclones & Reilsonable & Improved geometrical representation of system \\
\hline Liquid-liquid & Little & $\begin{array}{l}\text { Turbulence modeling }+ \text { modeling of interfa- } \\
\text { cial transport phenomena }+ \text { interaction of } \\
\text { hydrodynamics with chemical transtorma- } \\
\text { tion processes }\end{array}$ \\
\hline Fluid-solid & Reasonable & $\begin{array}{l}\text { Turbulence modeling }+ \text { refined models for } \\
\text { particle-particle and particle-wall interac- } \\
\text { tion }+ \text { prediction of flow regime transition } \\
+ \text { interaction of hydrodynamics with chemi- } \\
\text { cal transformation processes }\end{array}$ \\
\hline Packed beds & Some reasonable & Unit cell approach \\
\hline Fluidized beds & & $\begin{array}{l}\text { Extension to industrial fluidized beds with } \\
\text { complex geometries }\end{array}$ \\
\hline Gas-liquid-solid & & $\begin{array}{l}\text { Turbulence modeling + prediction of flow } \\
\text { regime transition + interaction of hydrody- } \\
\text { namics with chemical transformation } \\
\text { processes }\end{array}$ \\
\hline Trickle bed reactors & No & $\begin{array}{l}\text { Modeling on basis of unit cell approach }+ \text { de- } \\
\text { velopment of correspondence rules for } \\
\text { macroscopic system behavior }\end{array}$ \\
\hline Slurry reactors & Little & $\begin{array}{l}\text { Modeling of the effect of the solids phase on } \\
\text { interfacial transport phenomena }\end{array}$ \\
\hline Three-phase fluidized beds & Little & $\begin{array}{l}\text { Modeling of the effect of the solids phase on } \\
\text { interfacial transport phenomena }+ \text { develop- } \\
\text { ment of refined models for particle-particle } \\
\text { and particle-wall interaction }\end{array}$ \\
\hline
\end{tabular}




\section{Experimental Validation}

Experimental validation of CFD results is considered a prerequisite to paving the road for widespread acceptance of CFD in the chemical engineering community, especially in connection with multiphase flow applications. The authors do not intend to give here a complete review on available measuring techniques for single-phase and multiphase flows; only the more advanced techniques are briefly discussed. For an overview of the latest advances realized in noninvasive measurement of multiphase systems the interested reader is referred to Chaouki et al. (1997). The available experimental techniques can be classified according to the following aspects:

- Type of quantity measured

- Local or whole field measuring method

- Instantaneous or time-averaged quantities measured

- Intrusive or nonintrusive method

For the measurement of pressure, temperature, phase concentration, composition, and velocities in single-phase and multiphase systems a variety of experimental methods are available ranging from simple probe techniques to sophisticated whole field measuring methods. Thermal anemometry, electrical sensing techniques, light scattering and optical methods, electromagnetic wave techniques, and ultrasonic techniques have all been used to study complex fluid flows. For an overview of these techniques the interested reader is referred to Cheremisinoff (1986a).

Especially for multiphase systems flow visualization (Wen-Jei Yang, 1989; Merzkirch, 1987) can provide valuable initial information on the prevailing flow patterns and should at least always be considered as a first step. Of course, in applications that involve extreme conditions such as high temperature and/or pressure it is very difficult if not impossible to apply flow' visualization and other techniques should be considered. Here the use of cold flow models which permit visual observation might be considered as an alternative as an important first step to obtain (qualitative) information on the flow regime and associated flow pattern. Of course, multiphase flows exist such as dense gas-solid flows that do not permit visual observation and in such cases the application of idealized flow geometries should be considered. A well-known example in this respect is the application of so-called 2D gas fluidized beds to study gas bubble behavior (Rowe, 1971).

Due to the fact that the CFD approach usually leads to very detailed information on the temporal and spatial distribution of key variables it has also led to the development of very advanced experimental techniques to obtain the relevant experimental data. Some of these techniques, which reflect the recent progress in experimental fluid dynamics are discussed in more detail. Point-measuring tech- 
niques such as hot-wire anemometry (HWA) and laser Doppler anemometry (LDA) cannot give information on the instantaneous spatial structure of the flow. Especially in situations where one is interested in the dynamics of coherent structures, as encountered in, for example, turbulent single phase flows and dispersed gas-liquid two-phase flow, point-measuring techniques are inadequate and more advanced techniques are required.

a. Laser Doppler Anemometry. With LDA the velocities of flowing (small) particles can be determined from the frequency information contained in light scattered by the particles as they pass through a fringe or interference pattern. In single-phase flows these particles, which have to be sufficiently small to prevent separation effects, are deliberately added to the fluid (seeding of the flow). Since the intensity of the scattered light also contains information on the particle size LDA has also found application in atomized and dispersed flows to obtain particle size information (Farmer, 1972, 1974).

b. Particle Image Velocimetry. A very powerful class of velocity measuring techniques, termed pulsed light velocimetry, has become available in experimental fluid dynamics. In these techniques typically the motion of small, marked regions of a fluid is measured by observing the locations of the images of the markers at two or more times. The velocity is obtained from the ratio of the observed displacement $\Delta \bar{x}$ and the time interval $\Delta t$ separating the subsequent observations of the marker images:

$$
\bar{u}=\frac{\Delta \bar{x}}{\Delta t} .
$$

The marker fluid parcels can consist of small particles, bubbles, or droplets but can also be generated in situ by activating molecules constituting the fluid with laser beams, causing them to fluoresce (Gharib et al., 1985).

A very powerful method that belongs to the class of PLV techniques to obtain quantitative information on the instantaneous structure of the flow is particle image velocimetry (PIV) and its digital counterpart (DPIV). In these techniques a suitably chosen number of neutrally buoyant tracer particles are suspended in the fluid (usually a liquid) and subsequently a section of the flow is illuminated with the aid of a laser whereby the tracer particles become visible. By making highresolution video recordings of the illuminated section of the flow and subsequent processing of the digitized images using advanced statistical techniques (Westerweel, 1993) the instantaneous flow field of the filuid in the test section can be obtained. Due to this ability DPIV can provide detailed information on the dynamics of coherent flow structures, which is typically also the type of information that can be obtained from (advanced) CFD approaches. A critical aspect of PIV is the 
so-called seeding of the fluid with marker particles. For further details the interested reader is referred to the review paper of Adrian (1991) and Westerweel (1993) and the references cited therein.

PIV has also been applied to gas-liquid systems (Reese and Fan, 1994; Lin et al., 1996) to study the flow structure in bubble columns. A specific complication here is caused by the presence of the gas bubbles. On the basis of a prior knowledge of the size distribution of the tracer particles and the gas bubbles it is possible to discriminate bubbles from particles and thus phase-specific postprocessing of the images can be undertaken whereby both the flow pattern of the bubbles and the liquid in principle can be obtained. Particle image velocimetry has also been applied (Chen and Fan, 1992) to study the flow structure in 3D gas-liquid-solid fluidized beds.

c. Nuclear Magnetic Resonance Imaging. Although LDA and HWA are powerful methods for studying spatial and temporal variations of fluid velocities under a wide range of conditions, unfortunately these methods cannot be applied in optically opaque fluids (dense slurries, pastes. etc.) and flows which are inaccessible due to geometric constraints (i.e., porous media). In these situations magnetic resonance imaging (MRI) offers a convenient means to obtain detailed information on the flow (see, for example, Derbyshire et al., 1994).

MRI techniques can be broadly divided into magnitude and phase-based methods which respectively employ the magnitude and orientation of the local nuclear magnetization vector $M$ as tags which can be monitored in space and time. After creation and evolution of the tags the local magnetization state can be imaged. By creating subsequent images the displacement of fluid parcels over distances ranging from micrometers to centimeters occurring on a time scale varying from milliseconds to seconds can be registered. Especially for multiphase flows this type of measuring method possesses great potential. An example of the application of MRI to determine concentration and velocity profiles in solid suspensions in rotating geometries can be found in the paper of Corbett et al. (1995).

d. Laser-Induced Fluorescence. Another technicque that is particularly powerful in studies involving (turbulent) mixing processes. possibly in the presence of fast chemical transformations, involves fluorescent tracers together with laserinduced fluorescence (LIF) techniques (Gaskey et al., 1990; André et al., 1992). Through the application of laser sheet techniques it is possible to make measurements in a very thin cross section of the mixing zone. which is of crucial importance to validate closure models for turbulent reactive flow involving fast chemical reactions. A very recent development in this connection is the work by Dahm et al. (1995) where a very high temporal and spatial resolution was achieved. Recently Lemoine et al. 1996 used LIF and LDA to measure simultaneously species concentration and velocity in turbulent flows. 
e. Particle Tracking Methods. In particle tracking methods typically the motion of a particle is tracked in time using a set of detectors with a suitable geometrical arrangement where the behavior of the particle is (considered) representative for the phase under consideration. In literature several particle tracking techniques have been successfully employed to study multiphase flow systems such as bubble columns [Devanathan et al., 1990, using computer-aided radioactive particle tracking (CARPT)], fluidized beds [Larachi et al., 1995, 1996 using radioactive particle tracking (RPT)], and rotary mixers [Bridgwater et al., 1993, using positron emission particle tracking (PEPT)].

Devanathan et al. (1990) employed their CARPT technique to obtain information on the 3D path of a particle which from a hydrodynamic point of view can be considered as representative for the liquid phase in a bubble column. This technique thus offers the possibility to obtain important quantitative information on a key quantity of the Lagrangian representation of the flow. CARPT is essentially a particle tracking technique, in which the position of an essentially neutrally buoyant tiny particle, suspended in a two-phase gas-liquid flow, is registered as a function of time with a set of detectors with a proper geometrical arrangement around the bubble column. In principle, this technique can also be applied to other multiphase reactors such as gas fluidized or liquid fluidized beds and three-phase fluidized beds. Figure 15 shows the time-averaged streamlines and velocity vectors of the liquid phase obtained with the CARPT technique (Denavathan et al., 1990) in a 12 -in.-diameter bubble column operated at a superficial gas velocity of 0.105 $\mathrm{m} / \mathrm{s}$. From this figure the existence of a single circulation cell can be clearly inferred, which is contrary to the hypothesis of the existence of multiple circulation cells with cell height equal to the column diameter (Joshi and Sharma, 1979).

Larachi et al. (1996) applied their radioactive particle tracking technique to determine the time-averaged solids velocity in a three-phase fluidized bed. Techniques which are similar to CARPT, such as positron emission particle tracking and radioactive particle tracking, have been applied successfully to systems involving particulate solids.

f. Tomographic Techniques. Tomography refers to the cross-sectional imaging of an object from data collected by illuminating this object from many directions. This class of techniques is particularly powerful to obtain information on the spatial distribution of phases in a multiphase system in a nonintrusive manner. Thus tomographic techniques usually provide information on the flow morphology only and not on the motion of the flowing phases. X-ray tomography, $\gamma$-ray tomography, and electrical capacitance tomography constitute some of the better known tomography methods. Toye et al. (1995), for example, applied X-ray tomography to study liquid flow distribution in a trickle-bed reactor. Such measurements are considered very important in connection with the future development 

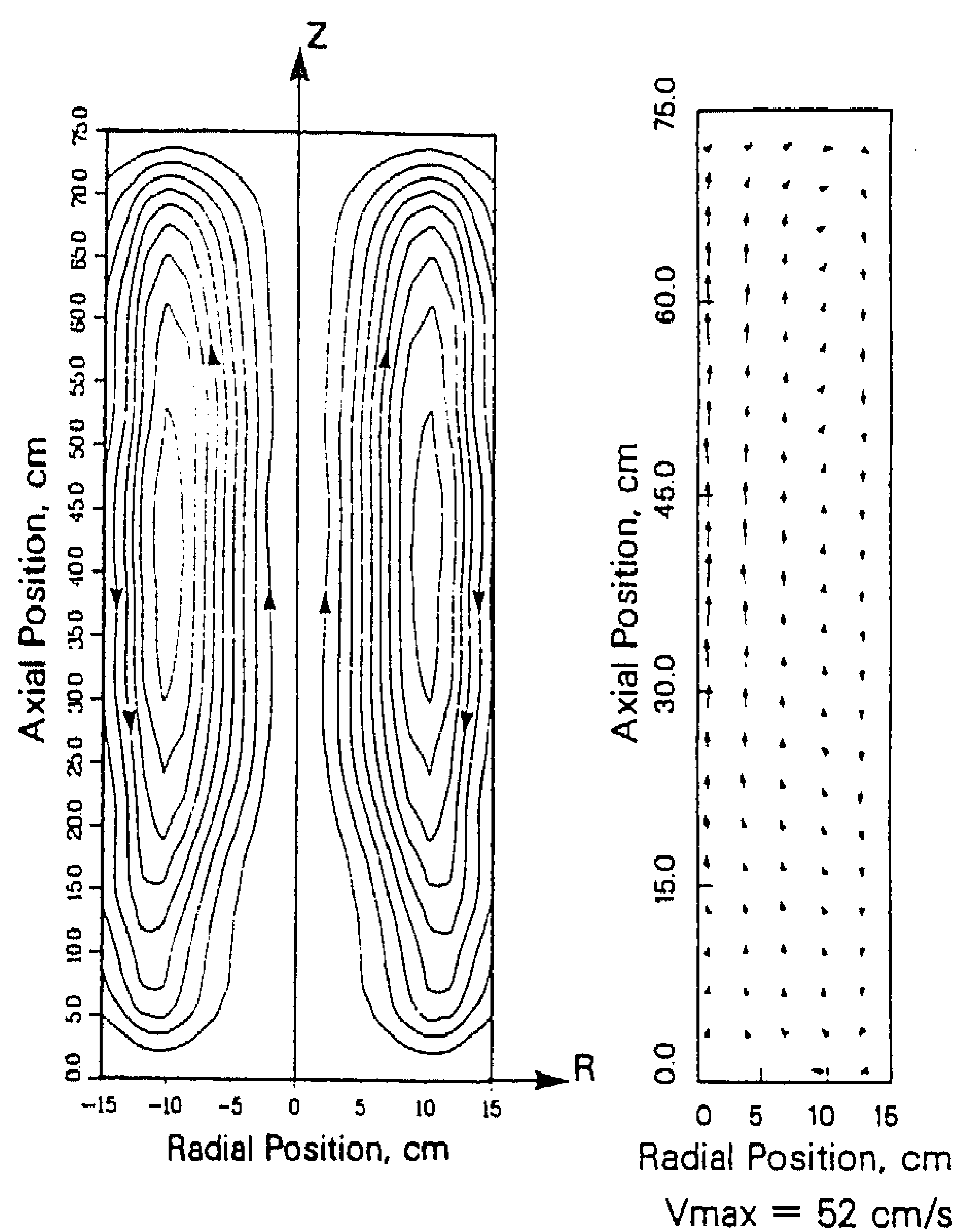

FIG. 15. Time-averaged streamlines and velocity vectors obtained by Devanathan ef al. $(1990$, using the CARPT technique. (From Chemical Engineering Science. Volume 45. Devanathan. N.. Moslemian, D.. and Dudukovicz. M.P. Flow mapping in bubble colunns using CARPT, pp. 2285-2291, copyright 1990. with permission from Elsevier Science.)

of fundamental hydrodynamic models for trickle-bed reactors. Martin et al. (1992) applied $\gamma$-ray tomography to determine the spatial distribution of cracking catalyst in a riser tube $(0.19 \mathrm{~m}$ diameter) of a cold-flow circulating fluidized bed and could assess the existence of the so-called core-annulus structure of the flow with a relative dense region near the wall of the riser tube.

g. $\gamma$-Ray Densitometr: A powerful method used to obtain information on the spatial solids distribution is $\gamma$-ray densitometry, which has been successfully applied in bubbling fluidized beds (Gidaspow et al., 1983) and circulating fluidized beds.

h. Miscellaneous Methods. For the measurement of key parameters, such as the solids volume fraction and solids velocity, in systems involving (dense) gas-solid two-phase flow a number of (specific) experimental techniques are available (Cheremisinoff. 1986b). Unfortunately, a number of powerful techniques such as 
LDA and PIV, which are applicable in single-phase systems, cannot be applied in dense gas-solid systems due to the fact that they are not optically transparent. Due to this fact a large number of specific techniques have been developed for these industrially important flows. Very often probe techniques have been used to measure locally the particle concentration and/or the particle velocity (see Nieuwland et al., 1996d, for a brief review). Due to the fact that probe techniques are usually intrusive, they may cause significant disturbances of the local flow behavior. which is especially disturbing in connection with validation of CFD models. Lim et al. (1995) have recently reviewed the hydrodynamics of gas-solid fluidization with particular emphasis on experimental findings and phenomenological modeling of bubbling and circulating fluidized beds. For gas-liquid systems a relatively large number of experimental techniques are available to obtain information on the gas holdup distribution. Also specific methods such as the "chemical method" should be mentioned here to determine the intefacial area by measuring the absorption rate of a gas in a suitably chosen absorption regime.

i. Concluding Remarks. Significant progress has been made in recent years with respect to the development of experimental techniques for both single-phase and multiphase flow applications. In Table IV a brief overview and classification of available experimental methods is presented. Clearly CFD has partly generated the driving force that has led to the development of some of these advanced experimental techniques and will continue to do so in the future. For multiphase flow there still exists a strong demand for nonintrusive experimental techniques which permit measurement of both time-averaged and fluctuating components of key quantities such as the local velocity and concentration of the phases present in the mixture.

\section{Selected Applications of CFD Work Conducted at Twente University}

In this section some selected results are reported on CFD work that has been carried out within the context of several Ph.D. programs completed at Twente University. Most of this work has been done on hydrodynamic modeling of multiphase systems.

\section{A. Two-Fluid Simulation of Gas Fluidized Beds}

Our previous studies have clearly shown that two-fluid models are able to capture much of the complex system behavior featured by gas fluidized beds. For example, Kuipers et al. (1991) and Nieuwland et al. (1996a) studied bubble forma- 
TABLE IV

OVERVIEW AND CLASSIFICATION OF EXPERIMENTAL METHODS

\begin{tabular}{|c|c|c|c|c|c|}
\hline $\begin{array}{l}\text { Measuring } \\
\text { Technique }\end{array}$ & $\begin{array}{l}\text { Quantity } \\
\text { Measured }\end{array}$ & $\begin{array}{l}\text { Point/Whole } \\
\text { Field Technique }\end{array}$ & $\begin{array}{l}\text { Instantaneous/ } \\
\text { Time-Averaged }\end{array}$ & $\begin{array}{l}\text { Intrusive/ } \\
\text { Nonintrusive }\end{array}$ & $\begin{array}{l}\text { Type of Flow } \\
\text { Application }\end{array}$ \\
\hline HWA & Velocity & Point & Instantaneous & Intrusive & $\begin{array}{c}\text { Single phase } \\
G+L\end{array}$ \\
\hline LDA & Velocity & Point & Instantaneous & Nonintrusive & $\begin{array}{l}\text { Single phase } \\
\text { G }+\mathrm{L} \\
\text { Multiphase } \\
\text { GL + dilute GS }\end{array}$ \\
\hline LDA & Particle size & Point & Instantaneous & Nonintrusive & $\begin{array}{l}\text { Single phase } \\
G+L \\
\text { multiphase } \\
\text { dilute GS }\end{array}$ \\
\hline PDA & Velocity & Point & Instantaneous & Nonintrusive & $\begin{array}{l}\text { Single phase } \\
\text { G+ }+ \\
\text { Multiphase } \\
\text { GL + dilute GS }\end{array}$ \\
\hline PDA & Particle size & Point & Instantaneous & Nonintrusive & $\begin{array}{l}\text { Single phase } \\
G+L \\
\text { Multiphase } \\
\text { dilute GS }\end{array}$ \\
\hline PIV & Velocity & Whole field & Instantaneous & Nonintrusive & $\begin{array}{l}\text { Single phase } \\
\text { G }+\mathrm{L} \\
\text { Multiphase } \\
\text { GL + GLS }\end{array}$ \\
\hline MRI & Velocity & Whole field & Instantaneous & Nonintrusive & $\begin{array}{l}\text { Single phase } \\
\qquad G+L\end{array}$ \\
\hline LIF & Concentration & Point & Instantaneous & Nonintrusive & $\begin{array}{c}\text { Single phase } \\
G+L\end{array}$ \\
\hline CARPT & Velocity & Whole field & Time-averaged & Nonintrusive & Multiphase GL \\
\hline RPT & Velocity & Whole field & Time-alveraged & Nonintrusive & $\begin{array}{l}\text { Multiphase } \\
\text { GS + GLS }\end{array}$ \\
\hline
\end{tabular}

tion at a single orifice in gas fluidized beds and have reported good agreement between experimentally observed bubble sizes and those obtained from two-fluid simulations. They have also shown that the two-fluid model, compared to existing approximate bubble formation models, in general, produced superior results. Figure 16 shows, as an example, the theoretically calculated growth, rise, and eruption of a single air bubble injected into a $2 \mathrm{D}$ air fluidized bed operated at minimum fluidization conditions, whereas Fig. 17 show's the experimentally observed and theoretically calculated bubble growth at a single orifice in a $2 \mathrm{D}$ air fluidized bed. From Fig. 17 it can be seen that the agreement between theory and experiment is satisfactory especially when one realizes that none of the parameter values required in the numerical simulation was derived from experimental data. Both theory and experiment indicate that especially during the initial stage of bubble formation a very sig- 

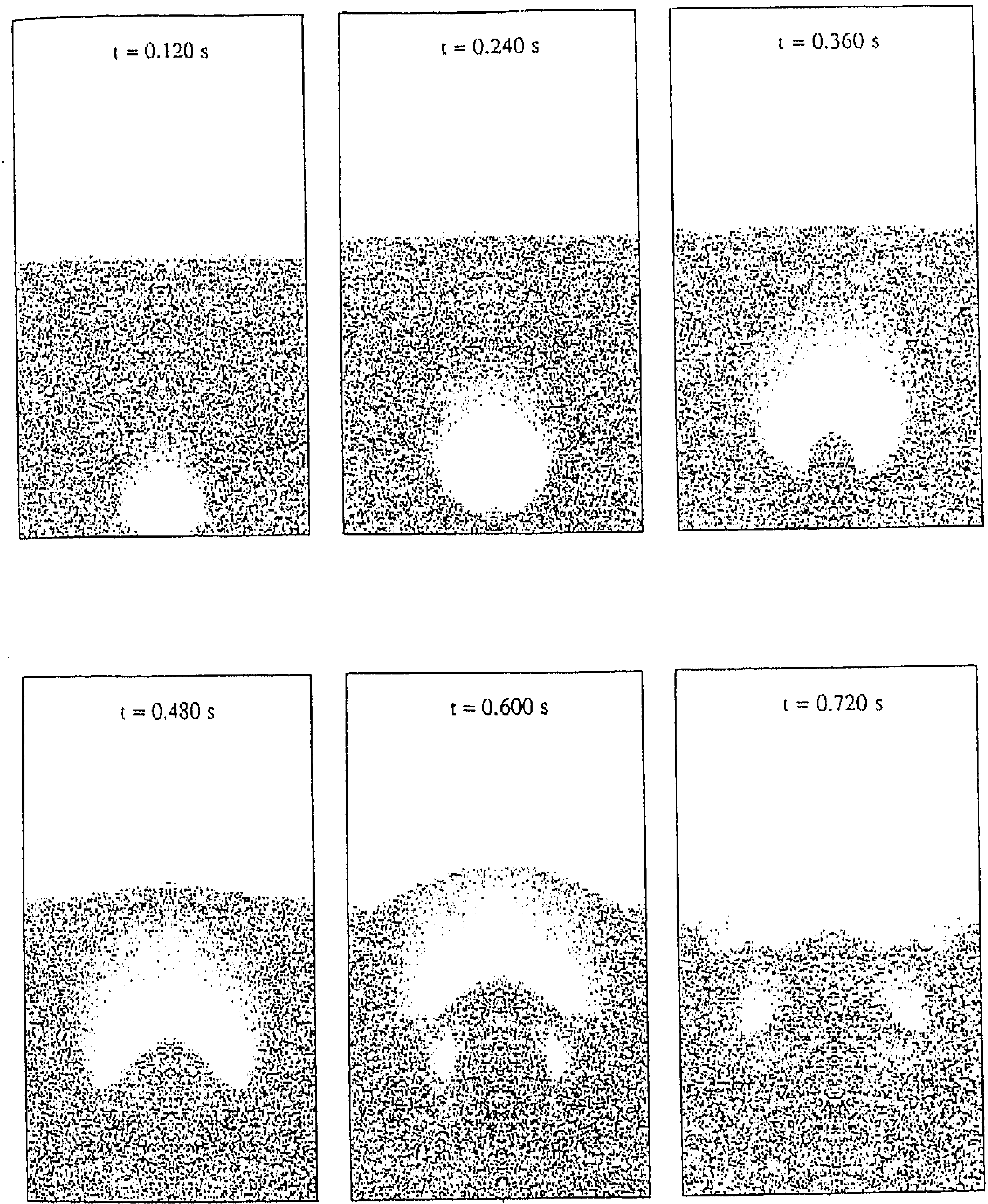

FIc. 16. Computer-generated solidity distribution showing the formation, rise, and eruption of a single bubble in a 2D gas fluidized bed. Physical properties of the particles: diameter, $500 \mu \mathrm{m}$ : density, $2660 \mathrm{~kg} / \mathrm{m}^{3}$. Bed dimensions: width, $0.58 \mathrm{~m}$; height, $1.0 \mathrm{~m}$.

nificant gas leakage from the bubble to the emulsion phase occurs. A quantitative description of this phenomenon is of considerable importance to gain insight in grid conversion phenomena in fluidized-bed reactors.

Nieuwland et al. (1995) studied bubble formation at a single orifice in a 2D gas fluidized bed operated at elevated pressure and found very good agreement between theory (two-fluid simulations) and experiment. Similar results were ob- 

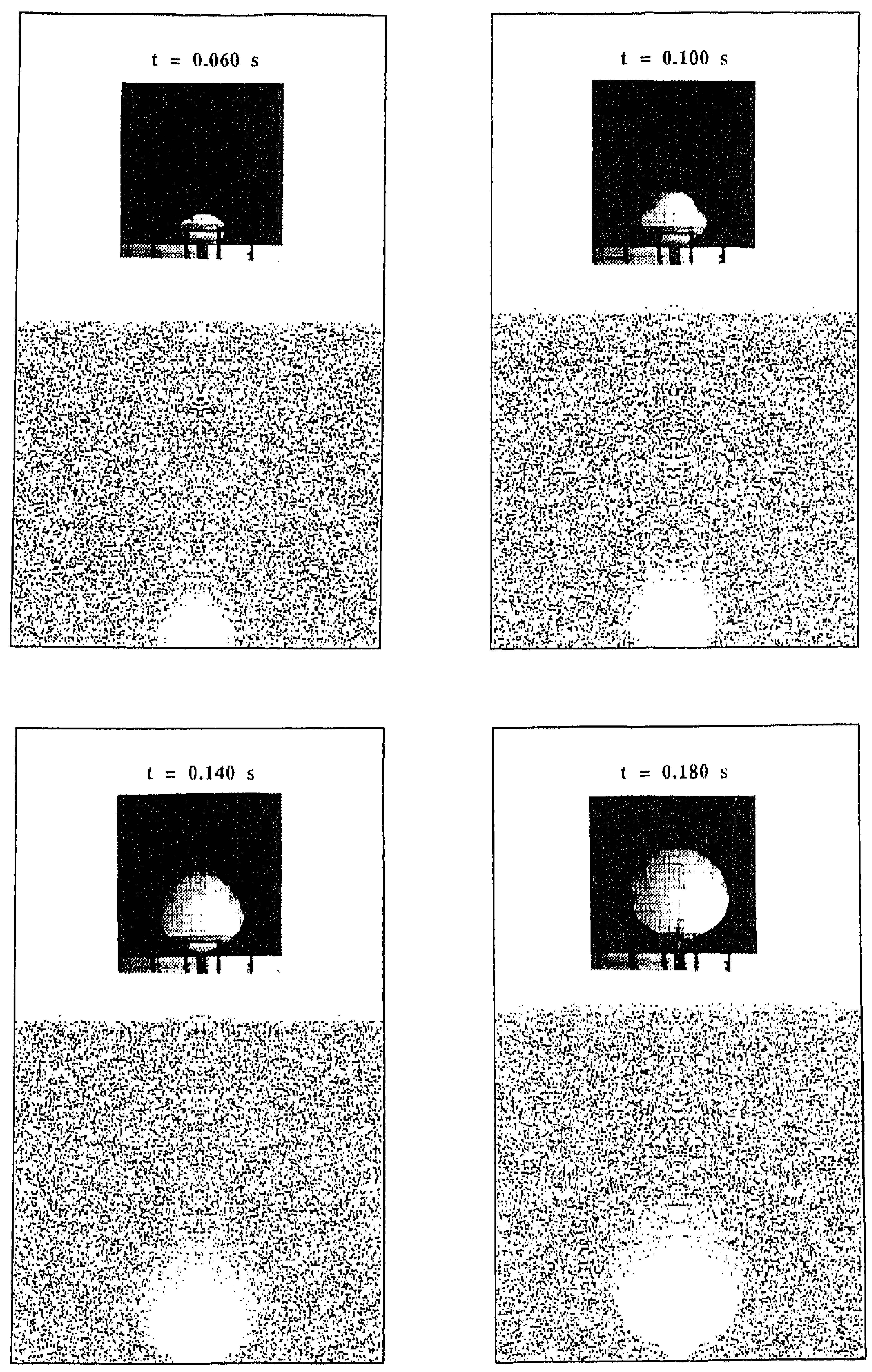

Fig. 17. Photographically observed and theoretically calculated bubble growth at a single orifice in a 2D gas fluidized bed. Physical properties of the particles: diameter, $500 \mu \mathrm{m}$ : density, 2660 $\mathrm{kg} / \mathrm{m}^{3}$. Bed dimensions: width, $0.58 \mathrm{~m}$; height, $1.0 \mathrm{~m}$. Injection velocity through orifice $10.0 \mathrm{~m} / \mathrm{s}$. 
tained by Huttenhuis et al. (1996) who studied the effect of gas phase density on bubble formation by injecting $\mathrm{He}$, air, or $\mathrm{SF}_{6}$ into an incipiently air-fluidized bed. Huttenhuis et al. (1996) also reported results of 3D simulations and found a clear effect of the front and back wall of their pseudo $2 \mathrm{D}$ bed. Their results stress the importance of $3 \mathrm{D}$ hydrodynamic modeling.

Two-fluid simulations have also been performed to predict void profiles (Kuipers et al., 1992b) and local wall-to-bed heat transfer coefficients in gas fluidized beds (Kuipers et al., 1992c). In Fig. 18 a comparison is shown between experimental (a) and theoretical (b) time-averaged porosity distributions obtained for a $2 \mathrm{D}$ air fluidized bed with a central jet (air injection velocity through the orifice: $10.0 \mathrm{~m} / \mathrm{s}$ which corresponds to $40 \mathrm{u}_{\mathrm{mf}}$ ). The experimental porosity distributions were obtained with the aid of a nonintrusive light transmission technique where the principles of liquid-solid fluidization and vibrofluidization were employed to perform the necessary calibration. The principal differences between theory and experiment can be attributed to the simplified solids rheology assumed in the hydrodynamic model and to asymmetries present in the experiment.

Figure 19 shows, as an example, the evolution and propagation of bubbles in a 2D gas-fluidized bed with a heated wall. The bubbles originate from an orifice near the heated right wall (air injection velocity through the orifice's $5.25 \mathrm{~m} / \mathrm{s}$, which corresponds to $2\left(u_{\mathrm{mf}}\right)$. The instantaneous axial profile of the wall-to-bed heat transfer coefficient is included in Fig. 19. From this figure the role of the developing bubble wake and the associated bed material refreshment along the heated wall, and its consequences for the local instantaneous heat transfer coefficient, can be clearly seen. In this study it became clear that CFD based models can be used as a tool (i.e., a learning model) to gain insight into complex system behavior.

\section{B. Discrete Particle Simllation of Gas Fluidized Beds}

Hoomans et al. (1996) developed a discrete particle model of gas fluidized beds where the 2D motion of individual, spherical particles was directly calculated from the forces acting on them, accounting for particle-particle and particle-wall interaction and interaction with the interstitial gas phase. Their collision model is based on the conservation laws for linear and angular momentum and requires, apart from geometrical parameters, a restitution coefficient and a friction coefficient. Techniques which are well known within the field of molecular dynamics were used to process a sequence of collisions. In fact, the model developed by Hoomans et al. can be seen as a combination of a so-called granular dynamics approach for the colliding particles and a CFD approach for the gas phase percolating through the particles. Discrete particle models offer certain distinct advantages over two-fluid models since they require no specific assumptions concerning the solids rheology. Furthermore, the incorporation of a particle size distribution can be accommodated with relative ease. 


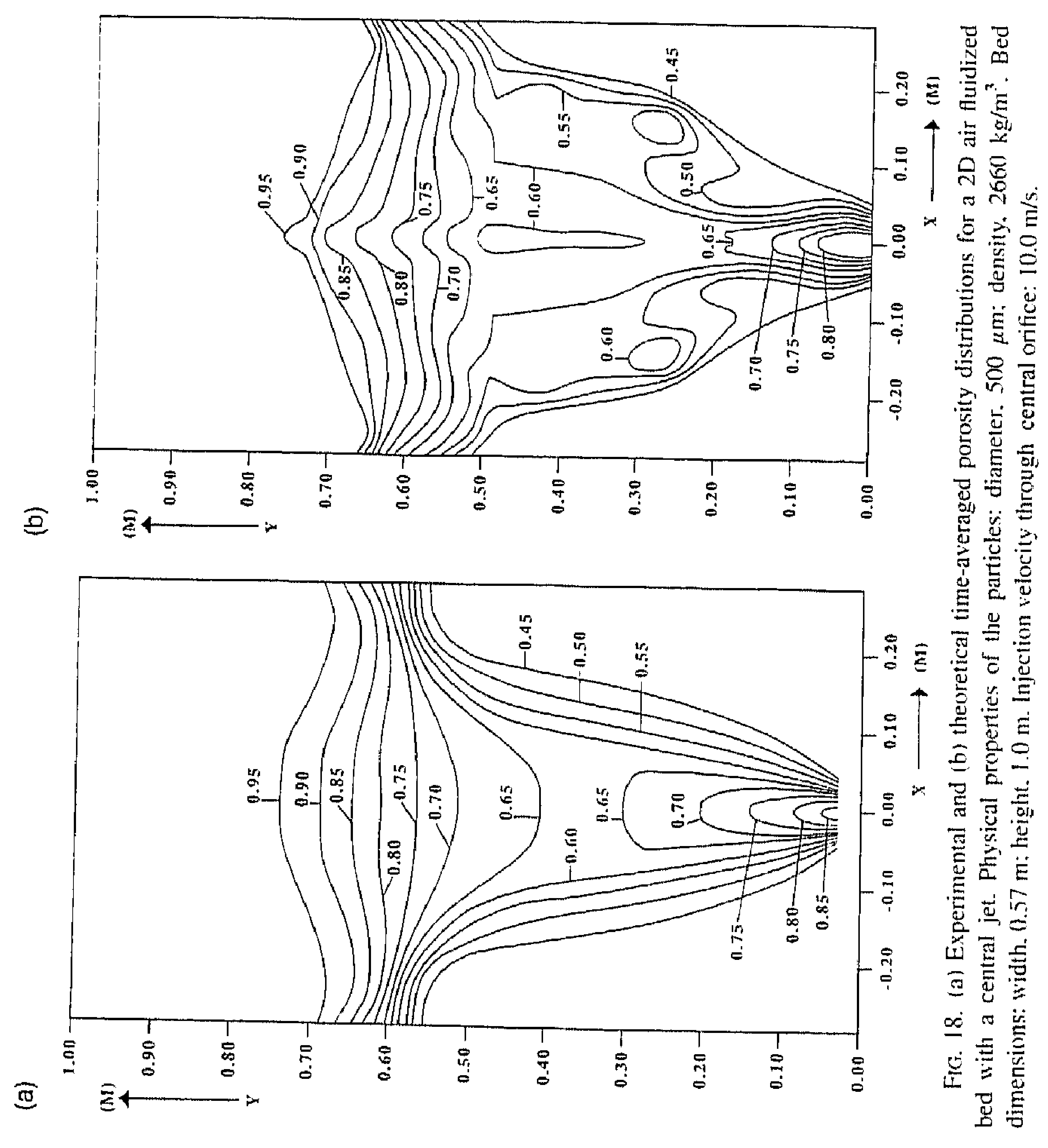



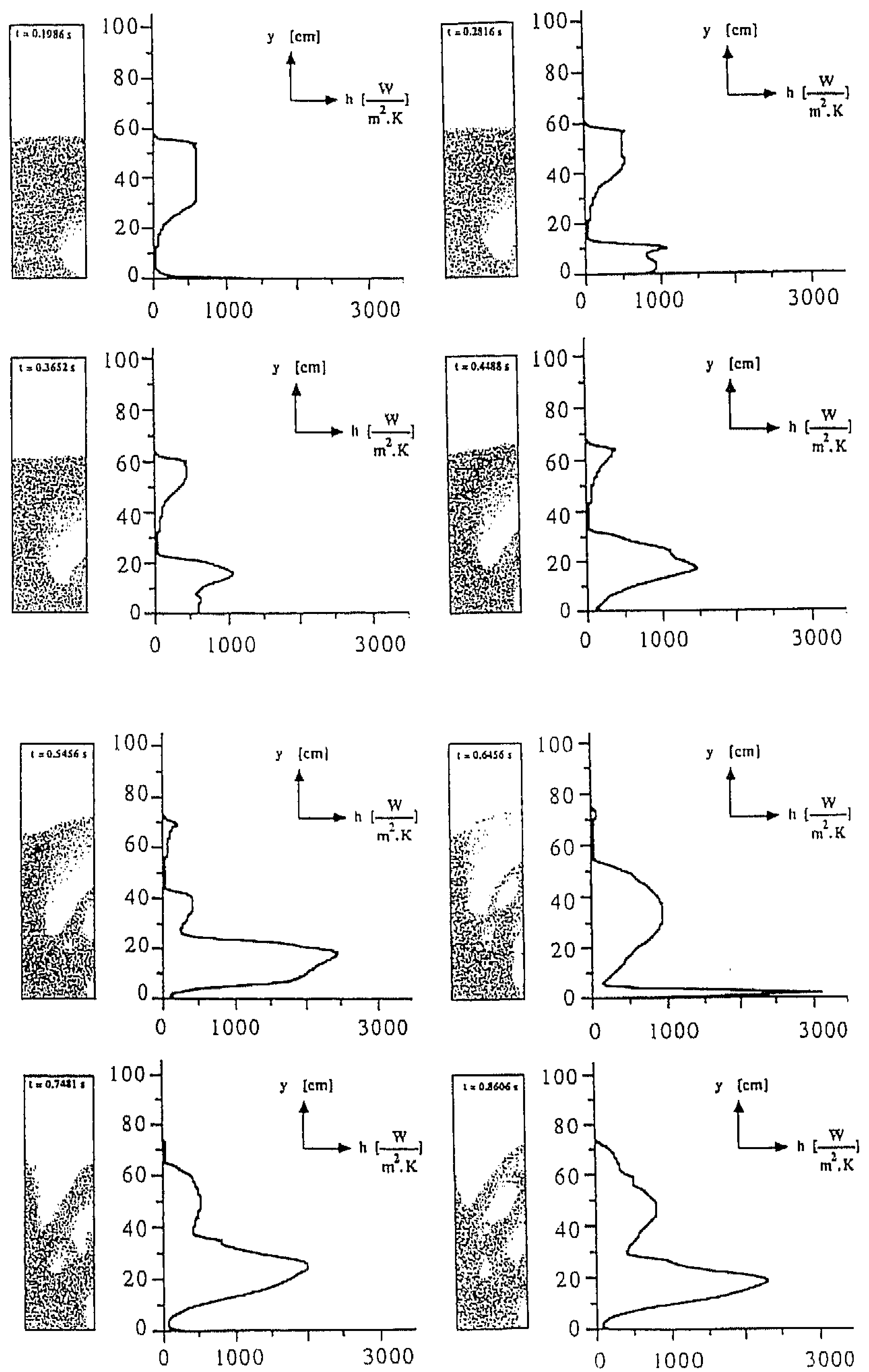

FIG. 19. Evolution and propagation of bubbles in a 2D air fluidized bed with a heated wall. The bubbles originate from an orifice near the heated right wall. Physical properties of the particles: diameter, $500 \mu \mathrm{m}$; density, $2660 \mathrm{~kg} / \mathrm{m}^{3}$. Bed dimensions: width, $0.285 \mathrm{~m}$; height, $1.0 \mathrm{~m}$. Injection velocity through orifice $5.25 \mathrm{~m} / \mathrm{s}$. 

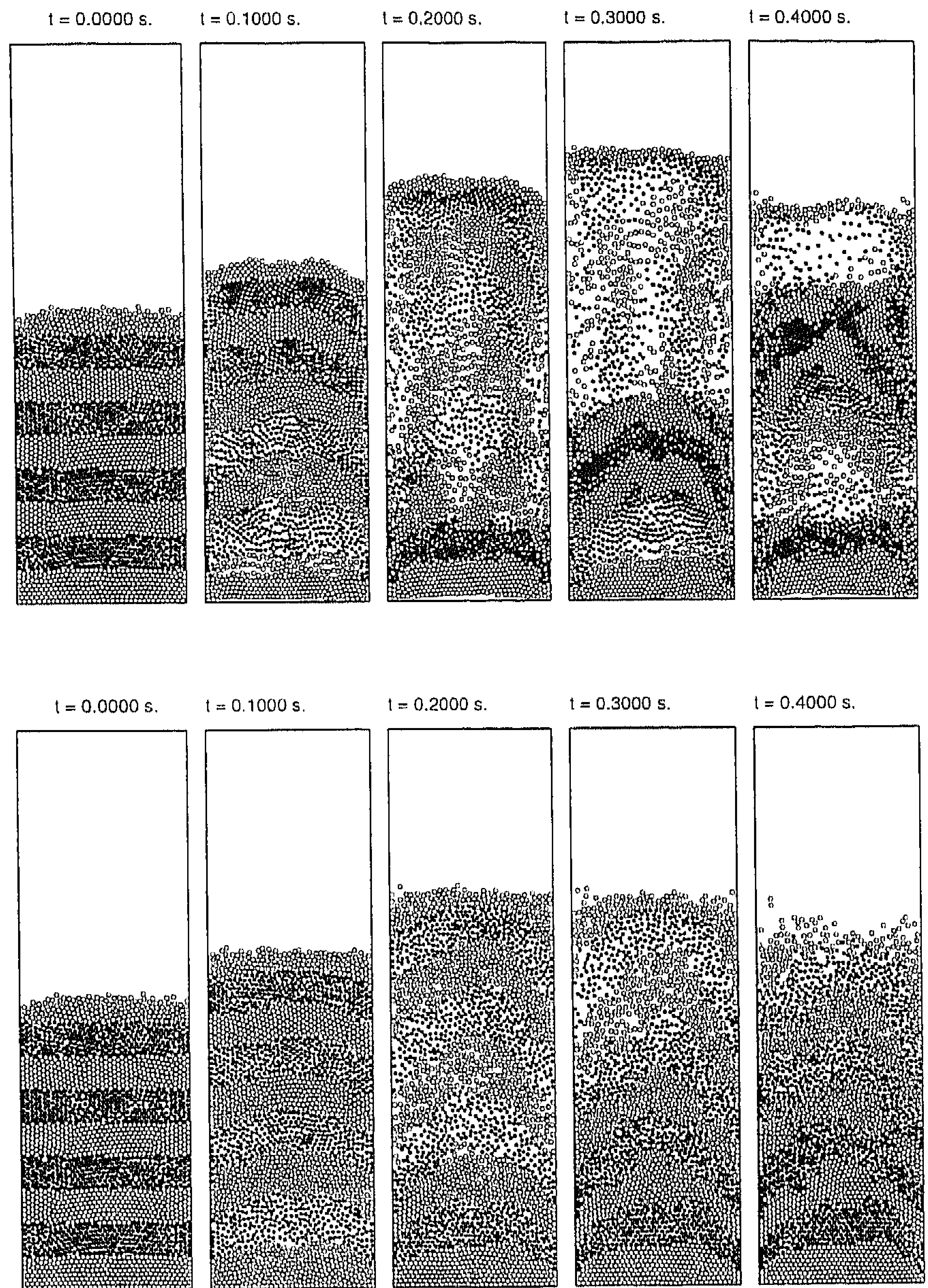

FIG. 20. Snapshots of particle configurations for the simulation of slug formation with homogeneous inflow conditions in a 2D gas fluidized bed. Top: Nonideal particles $\left(e=e_{\mathrm{w}}=0.9\right.$ and $\mu=\mu_{\mathrm{w}}=0.3$ ); bottom: ideal particles $\left(e=e_{\mathrm{w}}=1.0\right.$ and $\left.\mu=\mu_{\mathrm{w}}=0.0\right)$. 

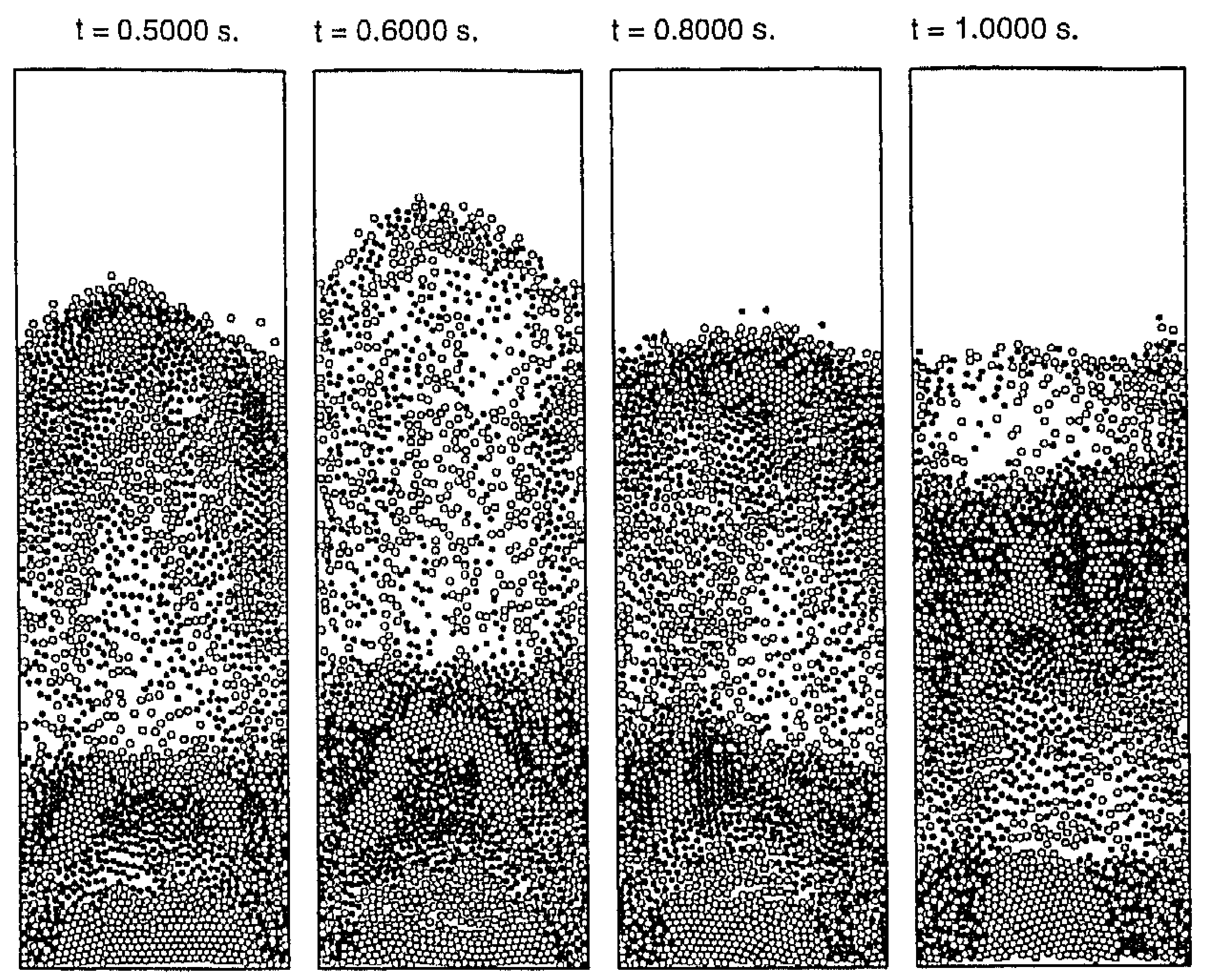

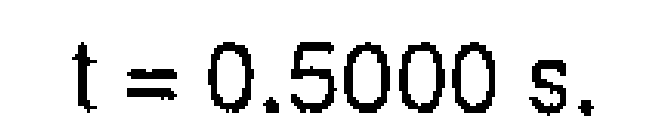

$$
t=0.6000 \mathrm{~s} .
$$

$t=0.8000 s$.
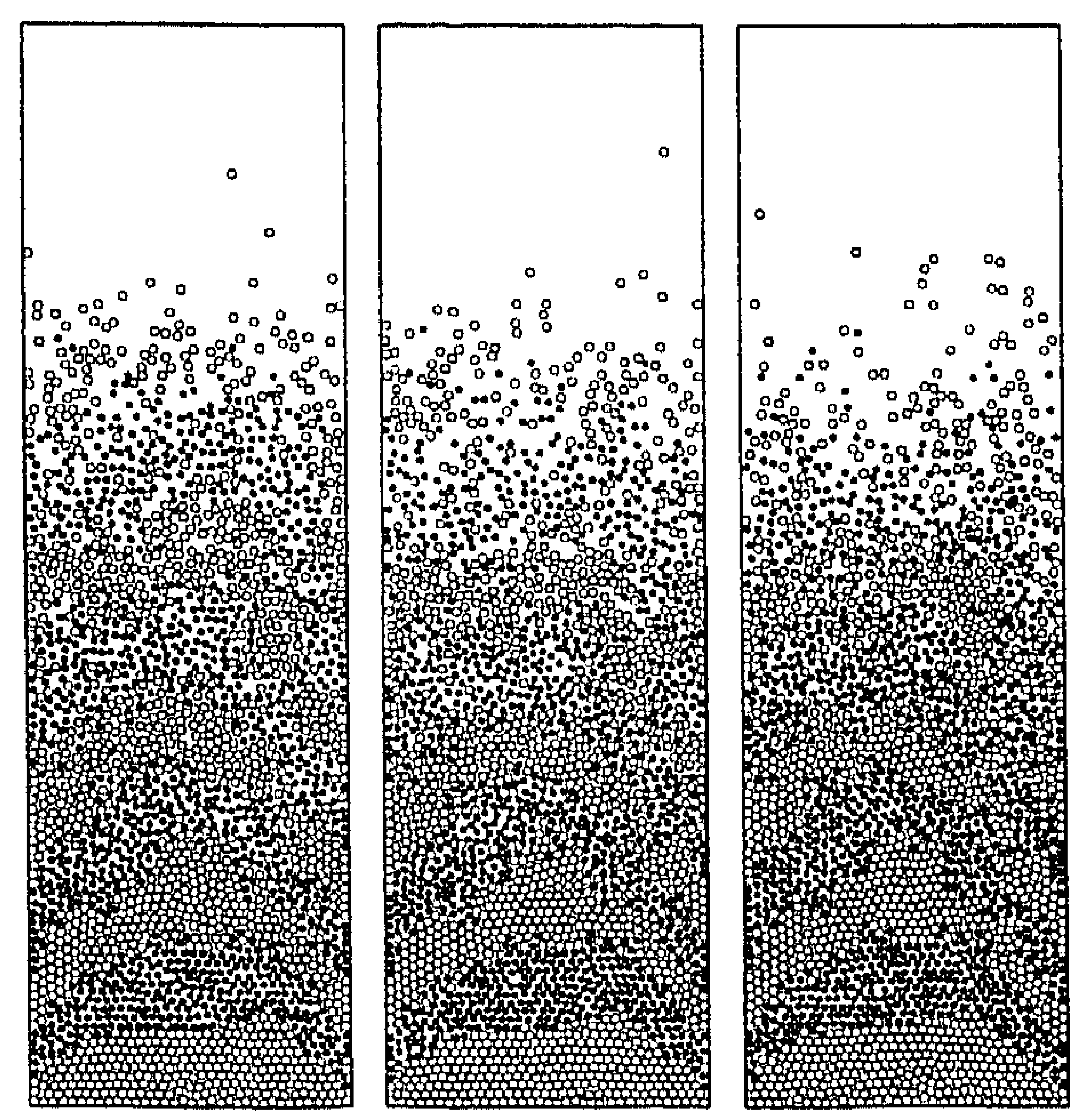

Fig. 20. Continued. $t=1.0000 s$.

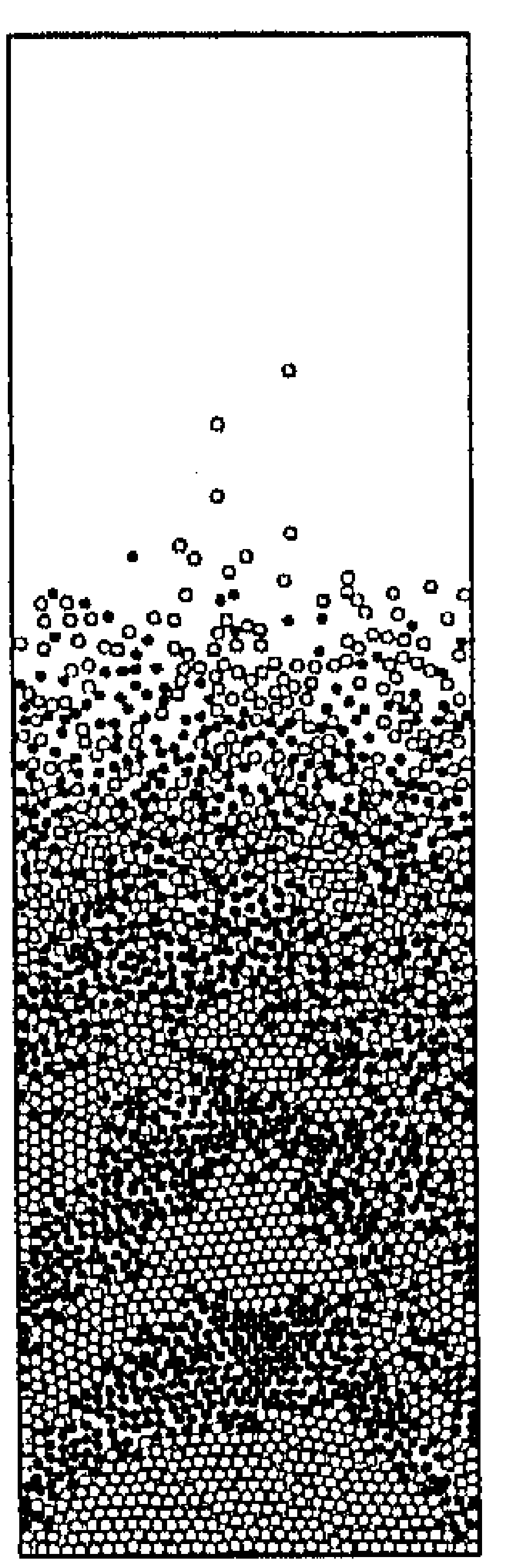


Figure 20 shows a sequence of particle configurations obtained from a discrete particle simulation of a two-dimensional gas fluidized bed where homogeneous inflow conditions were set at the bottom of the bed. The number of particles was 2400 whereas the diameter and the density of the particles were, respectively, $4 \mathrm{~mm}$ and $2700 \mathrm{~kg} / \mathrm{m}^{3}$. The particle configurations shown in Fig. 20 (top) represent the predicted bed behavior in case realistic values were used for the restitution and friction coefficient ( $e=0.9$ and $\mu=0.3$ ), whereas the particle configurations shown in Fig. 20 (bottom) refer to ideal particles $(e=1$ and $\mu=0)$. From this figure it can clearly be seen that the global system behavior, that is, the occurrence of bubbles and slugs and the associated mixing rate, differs significantly between the two cases. These results clearly indicate that dissipative processes on a microscale have a decisive effect on the global system dynamics.

\section{Circulating Fluidized Beds}

Nieuwland et al. (1996b,c) applied the kinetic theory of granular flow (KTGF) to study the hydrodynamics in a CFB riser tube. Their hydrodynamic model is based on the concept of two fully interpenetrating continua and consists of the two-fluid mass and momentum conservation equations describing the mean motion of the gas-solid dispersion and the granular temperature equation describing the fluctuating motion in the solid phase (Nieuwland, 1995). They used a modified Prandtl mixing length model to account for turbulent momentum transport in the gas phase. Nieuwland $e t$ al. assumed ideal (i.e., elastic) particle-particle collisions and nonideal particle-wall collisions because preliminary computations in which inelastic mutual particle collisions were assumed failed to produce the experimentally observed lateral solids segregation (Nieuwland et al., 1996b). This phenomenon is most likely due to the fact that the interaction between the fluctuating motions in the gas phase and solids phase is not accounted for in the model used by Nieuwland et al.

Figure 21a shows a comparison between computed and measured radial profiles of solids concentration for three superficial gas velocities $[U=7.5 \mathrm{~m} / \mathrm{s}, U=$ $10.0 \mathrm{~m} / \mathrm{s}$, and $U=15.0 \mathrm{~m} / \mathrm{s}$ ) at a constant solids mass flux $G_{\mathrm{s}}$ of $\left.300 \mathrm{~kg} /\left(\mathrm{m}^{2} \cdot \mathrm{s}\right)\right]$. The corresponding radial profiles of the axial solids velocity are shown in Figure $21 \mathrm{~b}$. The radial profiles of solids volume fraction and axial solids velocity were obtained with the aid of an optical probe developed by Nieuwland et al. (1996d) and similar in concept to the probe developed by Hartge et al. (1986). From Fig. 21a it can be seen that the radial solids segregation significantly increases with decreasing superficial gas velocity, which is in accordance with expectations. In general, their hydrodynamic model seems to underpredict the experimentally observed lateral solids segregation especially at the lowest superficial gas velocity of $7.5 \mathrm{~m} / \mathrm{s}$. However, it should be mentioned that in this case the average solids mass flux obtained from the experimental data showed a considerable positive $(48 \%)$ deviation form the imposed solids mass flux of $300 \mathrm{~kg} /\left(\mathrm{m}^{2} \cdot \mathrm{s}\right)$. In 
(a)

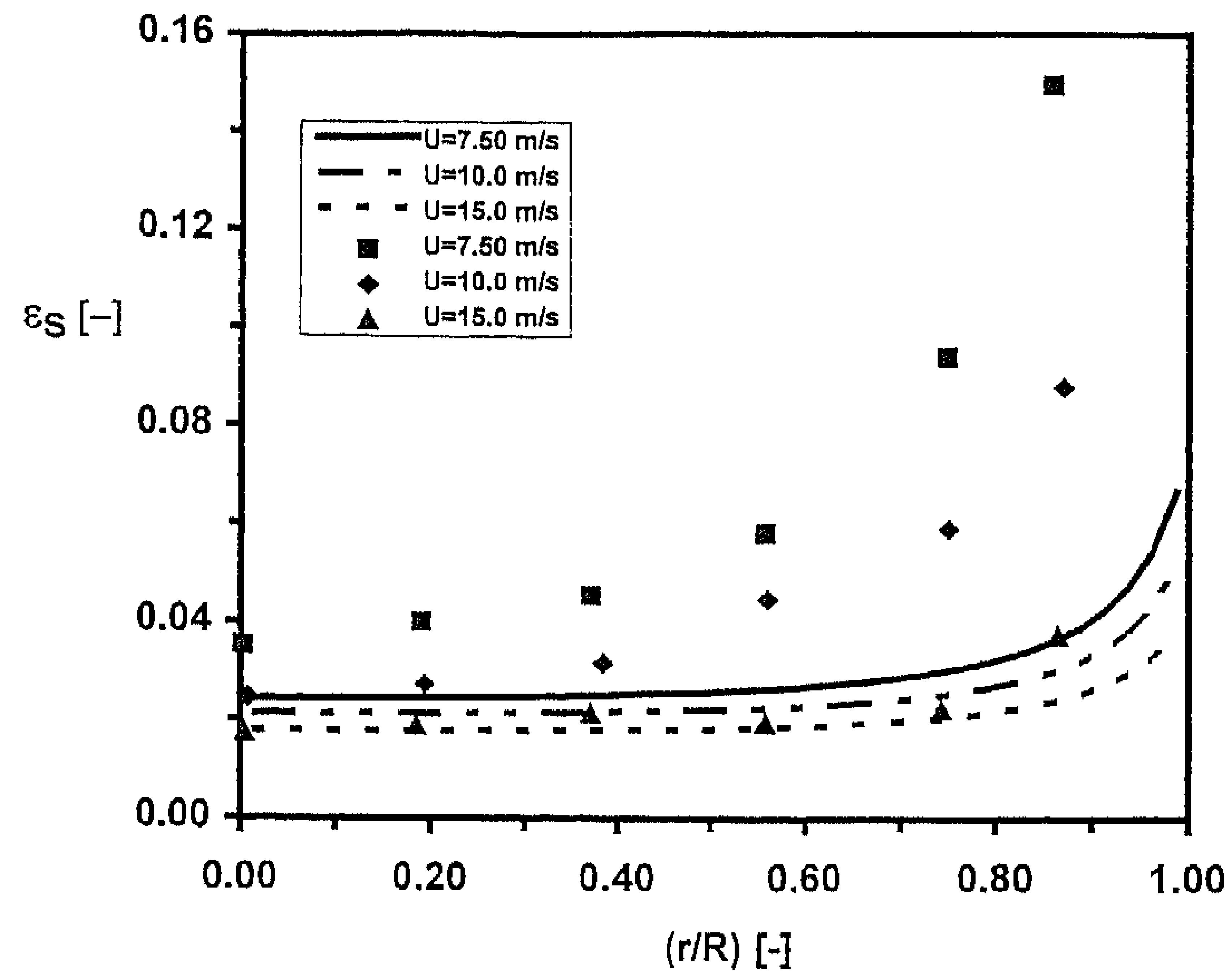

(b)

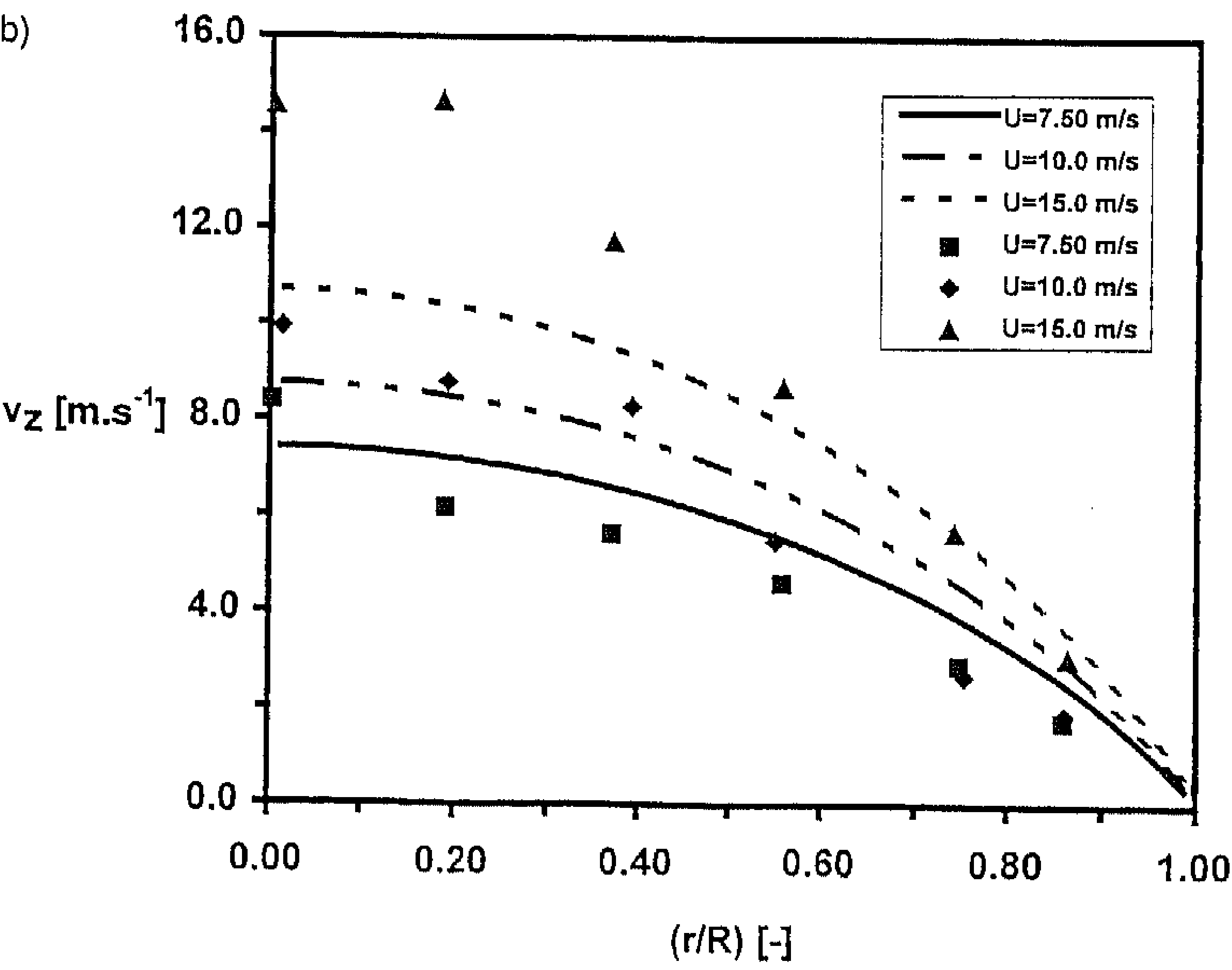

FIG. 21. Computed and measured radial protiles of (a) solids concentration and (b) axial solids velocity in a CFB riser for three superficial gas velocities $(U=7.5 \mathrm{~m} / \mathrm{s}, U=10.0 \mathrm{~m} / \mathrm{s}$. and $U=15.0$ $\mathrm{m} / \mathrm{s}$ ) at a constant mass flux $G_{\mathrm{s}}$ of $300 \mathrm{~kg} /\left(\mathrm{m}^{2} \cdot \mathrm{s}\right)$. Riser diameter $D=0.0536 \mathrm{~m}$, physical properties of the particles: diameter, $126 \mu \mathrm{m}$; density, $2540 \mathrm{~kg} / \mathrm{m}^{3}$. 
general, the agreement between theory and experiment is quite reasonable especially when it is borne in mind that their model contains no adjustable parameters. From Figure 21b it can be seen that the model predicts, in accordance with the experimental data, no solids downflow near the tube wall.

In addition Nieuwland et al. (1996b,c) compared their computational results with the experimental data reported by Bader et al. (1988). They performed experiments in a cold-flow CFB unit (riser tube: $D=0.304 \mathrm{~m} . L=10.0 \mathrm{~m}$ ) with FCC $\left(d_{\mathrm{p}}=76 \mu \mathrm{m}, \rho_{\mathrm{s}}=1714 \mathrm{~kg} / \mathrm{m}^{3}\right)$ as bed material and air as fluidizing agent. Preliminary calculations without using the KTGF failed to produce the experimentally observed lateral solids segregation, which demonstrates the importance of accounting for the collisional interaction between particles. Figure 22a shows a comparison between computed and measured radial profiles of solids concentration for a superficial gas velocity $U$ of $3.7 \mathrm{~m} / \mathrm{s}$ and a solids mass flux $G_{\mathrm{s}}$ of $98 \mathrm{~kg} /\left(\mathrm{m}^{2} \cdot \mathrm{s}\right)$. The corresponding radial profiles of the axial solids velocity are shown in Fig. 22b. Note that in this case the model predicts. in accordance with experiments, solids downflow near the tube wall.

In both figures the results of two additional computations are shown. In one of these calculations the constitutive equations derived by Ding and Gidaspow (1990) were used, which neglect the kinetic contributions in, respectively, the expressions for the solid phase shear viscosity and the solid phase pseudoconductivity. For the intermediate diameter riser $(D=0.304 \mathrm{~m})$ used by Bader et al. (1988) the total (i.e.. for both gas and solid phase) momentum transport in the radial direction is dominated by the turbulent contribution in the gas phase. which explains the small effect of neglecting the aforementioned kinetic contributions in the solid phase. In the other additional calculation shown in Figs 22a and $b$ the turbulent contribution in the expression for the gas phase shear viscosity was neglected. In this case the solids downflow near the tube wall is significantly overpredicted, which demonstrates the importance of accounting for macroscopic turbulent transport in the gas phase.

The results presented so far in this section correspond to the regime of fully developed riser flow. Kuipers and van Swaaij (1996) applied the KTGF-based model developed by Nieuwland et al. (1996b.c) to study the effect of riser inlet configuration on the (developing) flow in CFB riser tubes and found that the differences in computed radial profiles of hydrodynamic key variables (i.e.. gas and solids phase mass fluxes) rapidly disappear with increasing elevation in the riser tube.

\section{Bubble Columns}

Bubble columns find frequent application in the process industries due to their relatively simple construction and advantageous properties such as excellent heat transfer characteristics to immersed surfaces. Despite their frequent use in a variety of industrial processes. many important fluid dynamical aspects of the prevailing gas-liquid two-phase flow in bubble columns are unfortunately poorly un- 

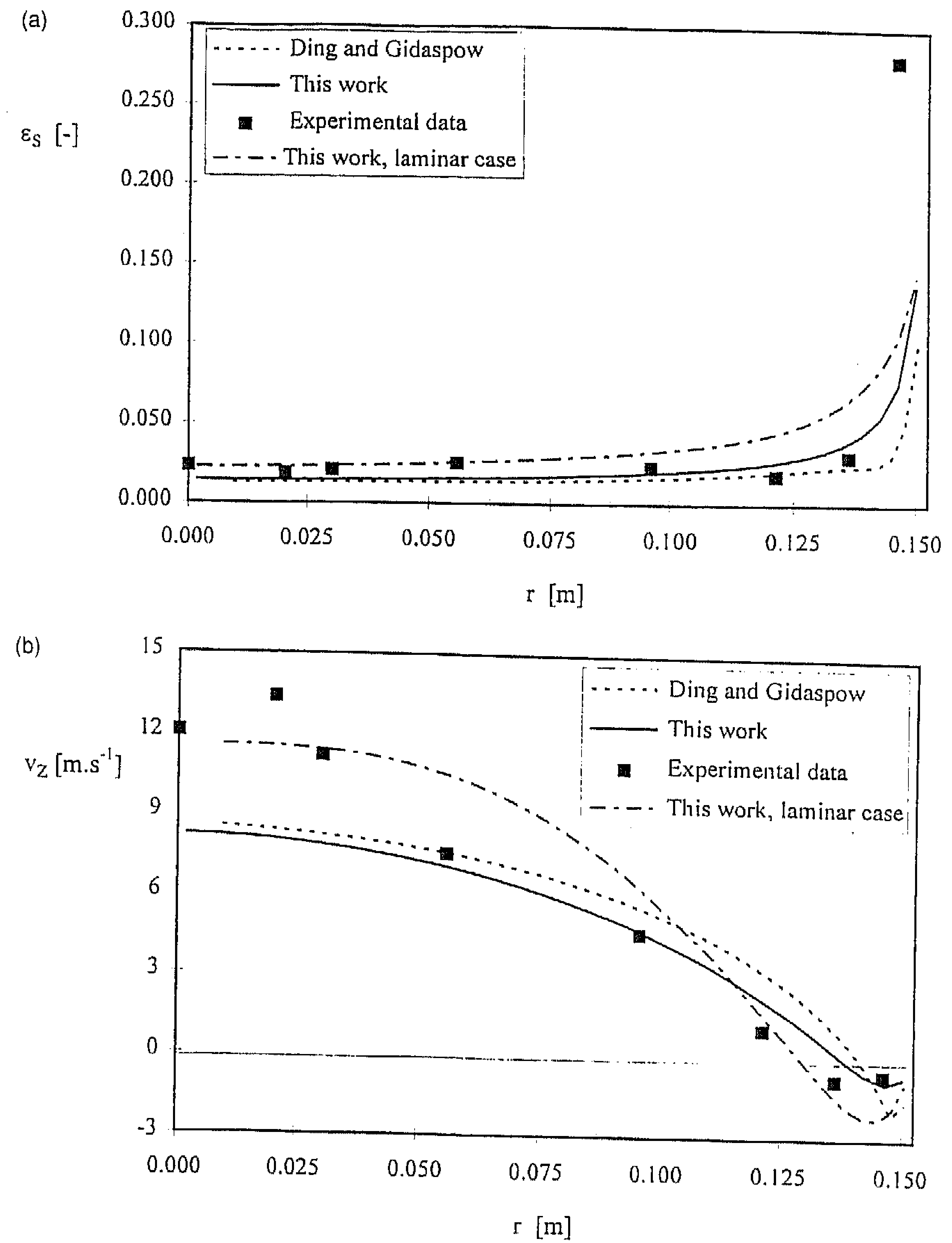

FIG. 22. Computed and measured (Bader et al., 1988) radial protiles of (a) solids concentration and (b) axial solids velocity in a CFB riser for a superficial gas velocity $U$ of $3.7 \mathrm{~m} / \mathrm{s}$ and a mass flux $G_{i}$ of $98 \mathrm{~kg} /\left(\mathrm{m}^{2} \cdot \mathrm{s}\right)$. Riser diameter $D=0.304 \mathrm{~m}$, physical properties of the particles: diamerer. 76 $\mu \mathrm{m} ;$ density, $1714 \mathrm{~kg} / \mathrm{m}^{3}$. 
derstood. This relative unsatisfactory state of the art has led to an increased interest in recent years in development of advanced experimental tools (Devanathan $e t$ al., 1990; Lin et al., 1996) and detailed modeling of bubble columns on the basis of the hydrodynamic equations of change (see Sect. IV, B,1).

Depending on the magnitude of the superficial gas velocity, three flow regimes (Fan, 1989) can be distinguished, namely, (1) dispersed bubble regime, (2) vortical-spiral flow regime, and (3) turbulent flow regime. In the dispersed bubble or homogeneous regime, relatively small nearly spherical gas bubbles are present with a more or less uniform size, whereas in the vortical-spiral and turbulent flow regimes besides the small gas bubbles relatively (very) large gas bubbles are seen with a size-dependent characteristic shape. In the next two subsections two different models will be discussed which apply to a situation where only many small (spherical) gas bubbles are present and a situation where a few large (nonspherical) gas bubbles are present.

\section{Discrete Bubble Model}

Delnoij et al. (1997a) developed a detailed hydrodynamic model for dispersed gas-liquid two-phase flow based on a mixed Eulerian-Lagrangian approach. Their model describes the time-dependent motion of small, spherical gas bubbles in a bubble column operating in the homogeneous regime where all relevant forces acting on the bubble (drag, virtual mass, lift, and gravity forces) were accounted for. Direct bubble-bubble interactions were accounted for via an interaction model, which resembles the collision approach followed by Hoomans et al. (1996) to model gas fluidized beds. Delnoij et al. (1997a) simulated two experiments reported by Becker et al. (1994), one for a large superficial gas velocity and another one for a small superficial gas velocity. The geometry used by Becker et al. comprises a pseudo two-dimensional bubble column equipped with a gas distributor section containing five, individually controllable porous plates. Becker et al. fed air to the column through only one of the five porous plates during their experiments.

a. Results for a Large Superficial Gas Velocity: Figure 23 shows a sequence of plots showing both the instantaneous configuration of the bubbles and the associated flow field in the liquid phase. In accordance with visual observations reported by Becker $e$ al. the model correctly predicts a powerful liquid circulation, which pushes the bubble swarm firmly toward the left wall of the column. This strong liquid circulation is induced by the large number of bubbles that rise through the column.

b. Results for a Small Superficial Gas Velocity. At lower superficial gas velocities Becker et al. (1994) observed a remarkable transition in the liquid phase flow pattern in their bubble column. Contrary to the experiment with a large superficial 
gas velocity, in this case the bubble swarm was found to move upward in a meandering manner. Several liquid circulation cells were reported that changed their location and size continuously. The flow was observed to be highly dynamic with a period of oscillation of the plume of approximately $41 \mathrm{~s}$. Figure 24 shows a sequence of plots showing both the instantaneous configuration of the bubbles and the associated flow field in the liquid phase. The meandering behavior and main liquid phase flow characteristics reported by Becker et al. could clearly be reproduced by the model developed by Delnoij et al. However, the period of oscillation calculated by their model was approximately $30 \mathrm{~s}$ and was felt to be due to the two-dimensional nature of the model.

c. Effect of Column Aspect Ratio on Flow Structure. Delnoij et al. also investigated the effect of the bubble column aspect ratio on the prevailing flow structure and compared their computational results with experimental data reported by Chen et al. (1989). A transition in the gas-liquid flow pattern was predicted in case the aspect ratio of the column changed from two to four. For an aspect ratio of two the Gulfstream type of liquid circulation was predicted, whereas for an aspect ratio of four a highly dynamic liquid flow pattern with multiple vortices was computed. From computer animations it could be seen that these vortices were generated at the free surface. Furthermore, these vortices were found to be positioned staggered with respect to each other in the column. In part these computational results were supported by Chen's findings. The only major difference with the experimental observations of Chen et al. was the fact that the aforementioned transition already occurred at an aspect ratio of one. Again this discrepancy is most likely due to the two-dimensional nature of the model developed by Delnoij et al. Finally as an illustration in Figure 25 a few plots of instantaneous bubble configurations are shown for bubble columns with aspect ratios ranging from 4.8 to 11.0. From these figures it can be clearly seen that the flow structure is significantly affected by the column aspect ratio. In Figure 25 for one case $(L / D=7.7)$ the time-averaged liquid velocity is also shown. The existence of the characteristic large-scale circulation with liquid uptlow in the center of the column and down-flow near the walls can be recognized clearly.

\section{Volume of Fluid (VOF) Model}

Delnoij et al. (1997b) developed a computer code based on the volume of fluid (VOF) method (Hirt and Nichols, 1981; Nichols et al., 1980) to describe the dynamics of single gas bubbles rising in a quiescent Newtonian liquid. They were able to show that the predicted bubble shape and associated flow patterns induced in the liquid phase could be predicted very well with the VOF method over a wide range of Eotvös (EO) and Morton (M) numbers (see Sec. IV,B,1 for the definitions of Eo and $\mathrm{M}$ ). In Fig. 26 the formation and rise of single gas bubbles emanating from a central orifice is shown for various values of the Eotvös and Morton num- 

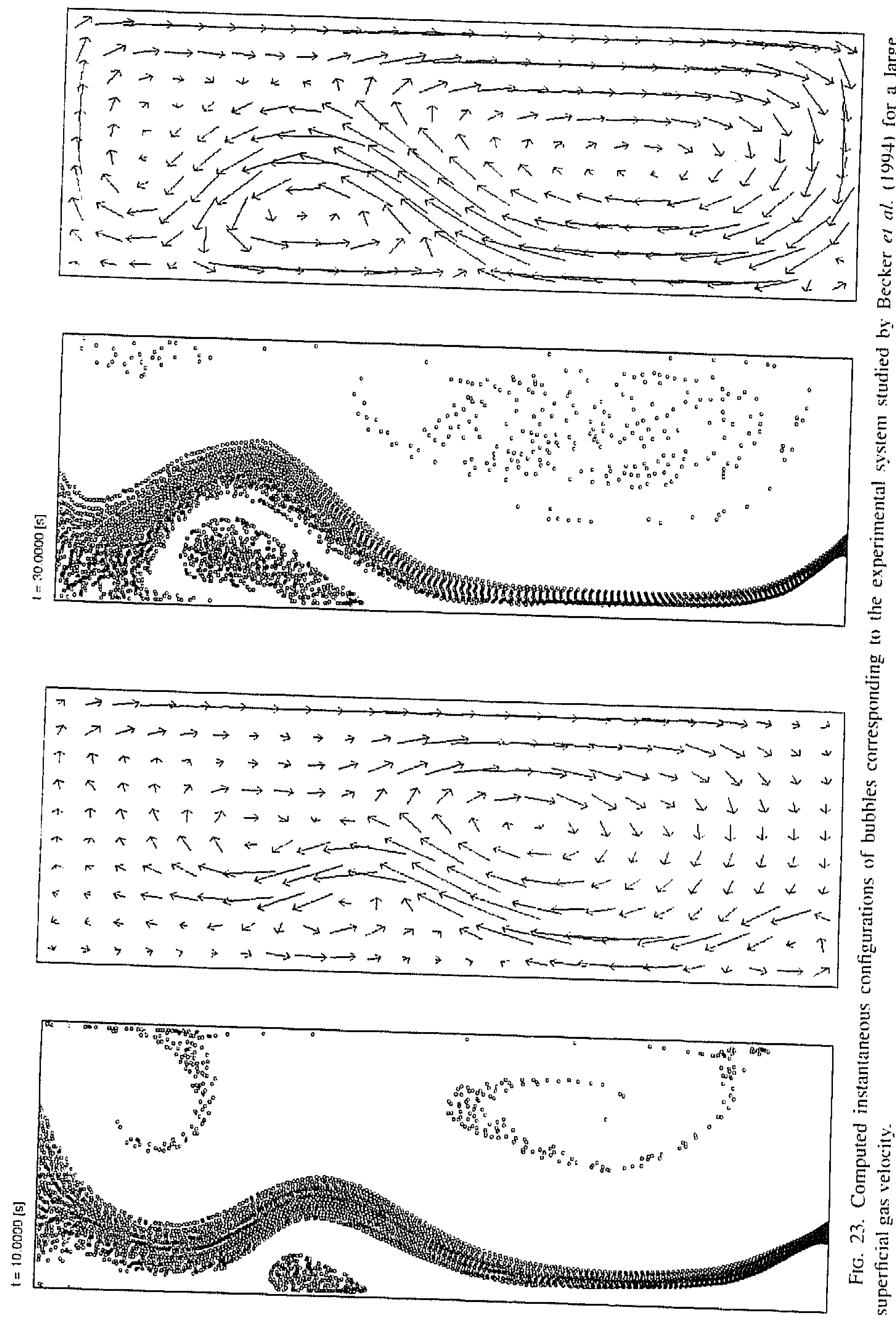


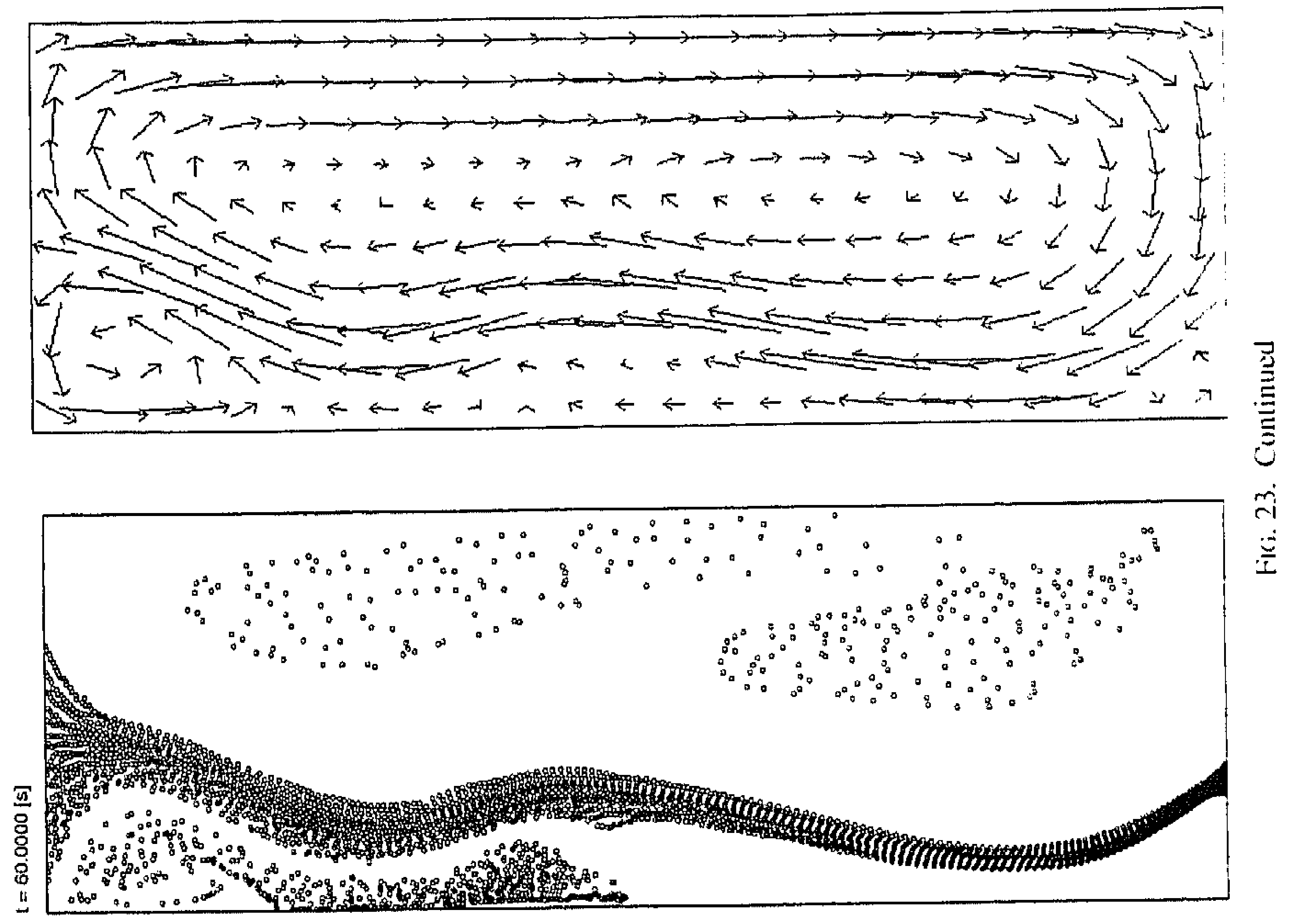



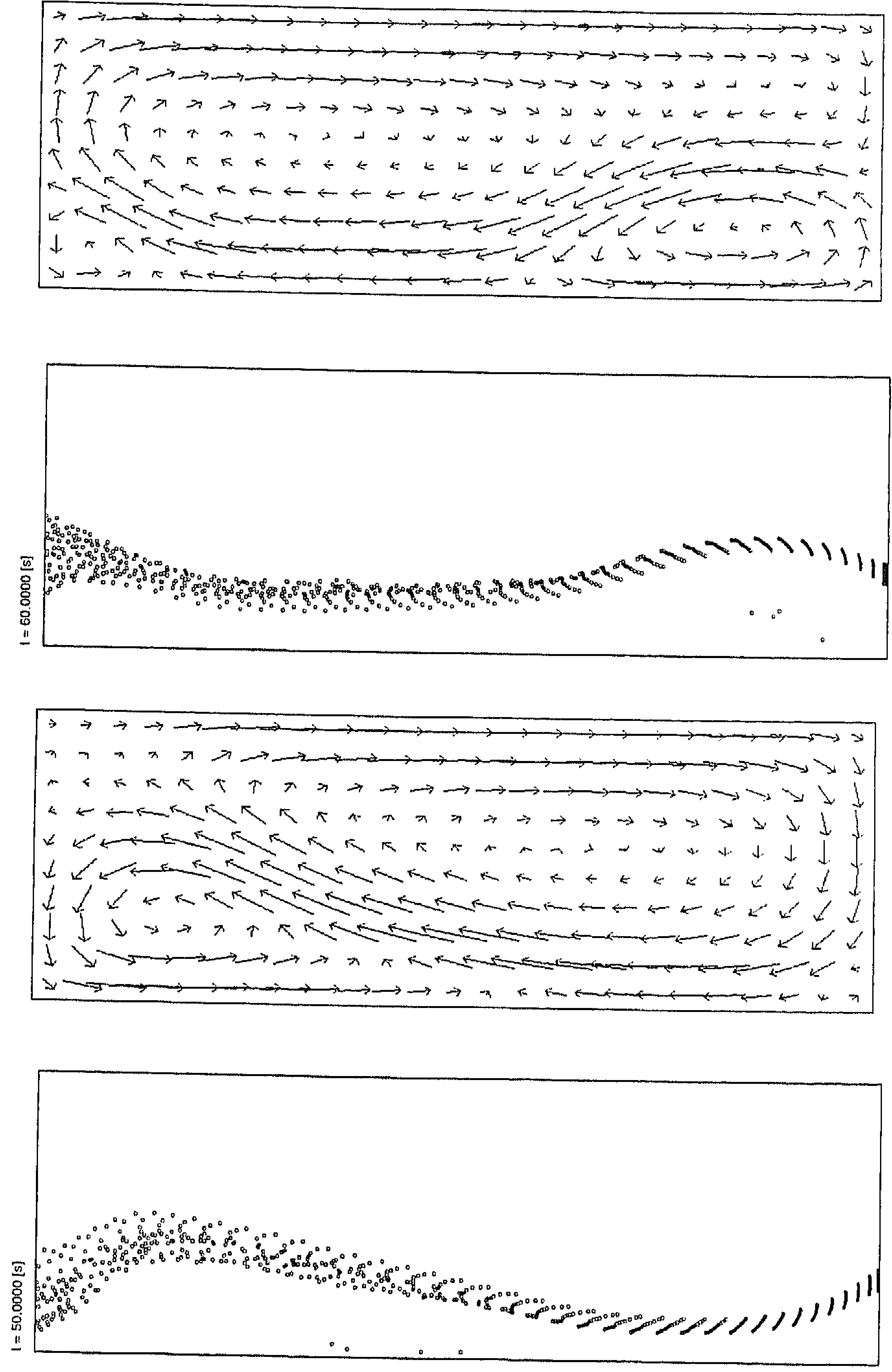

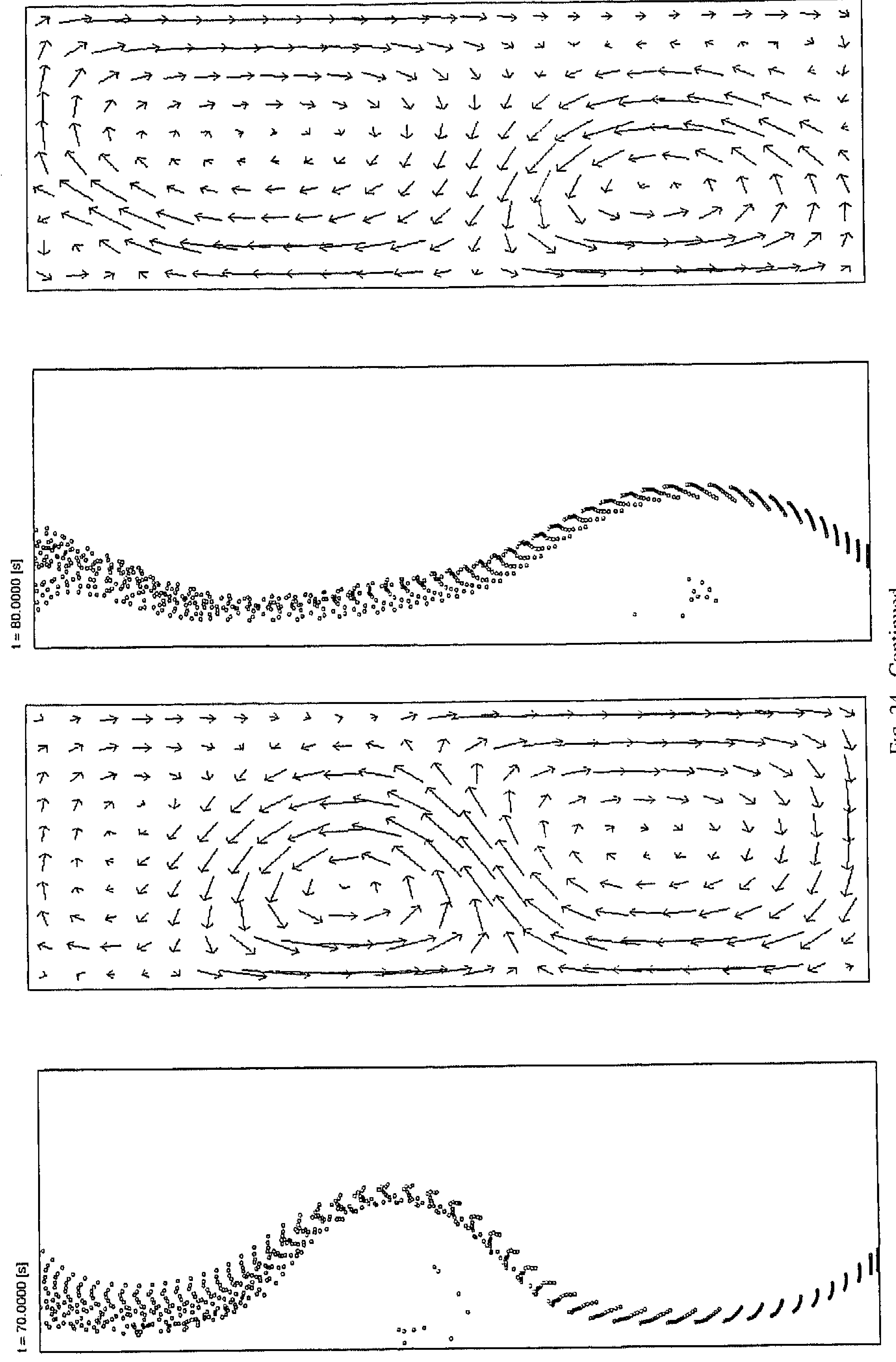

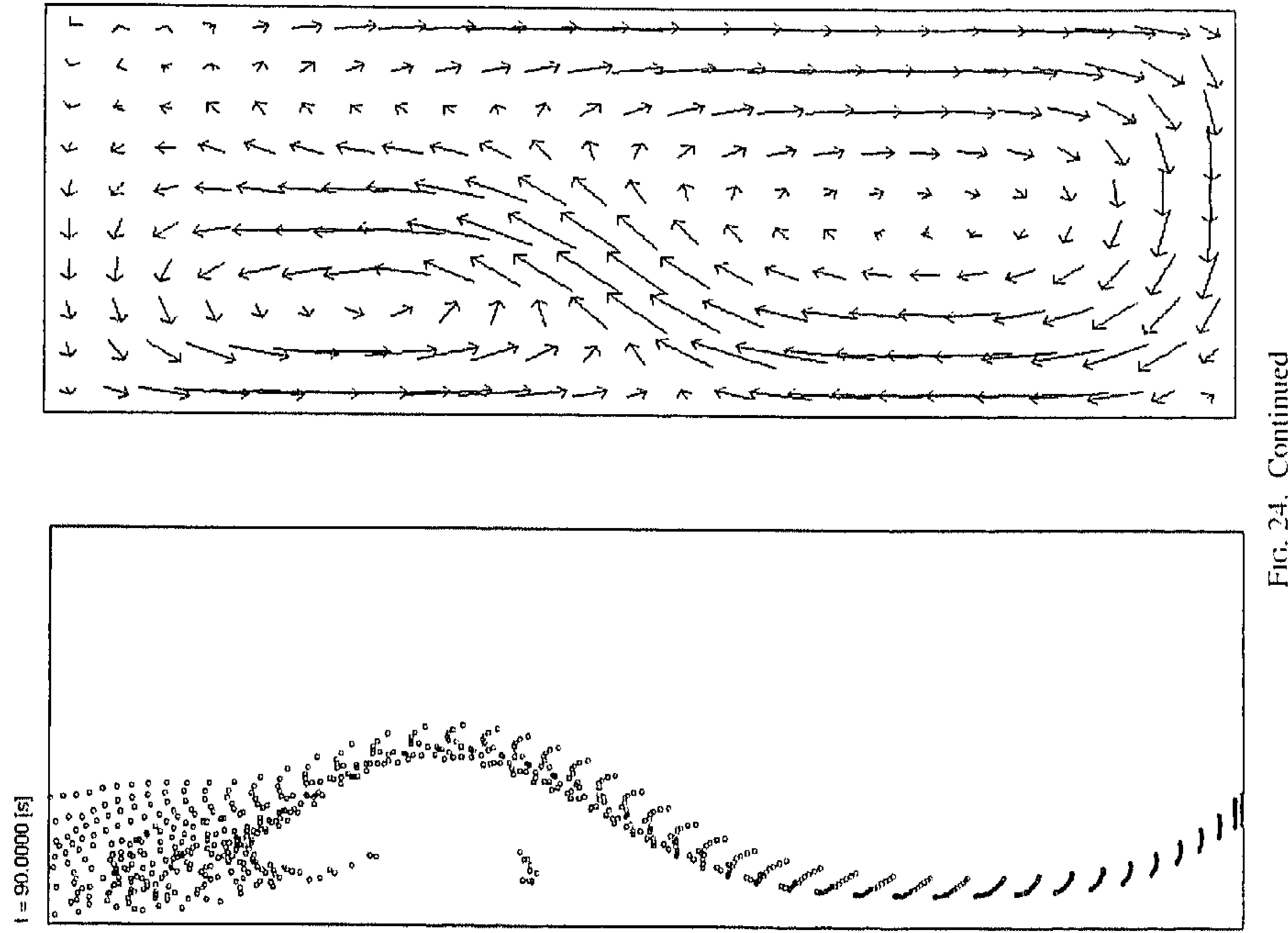

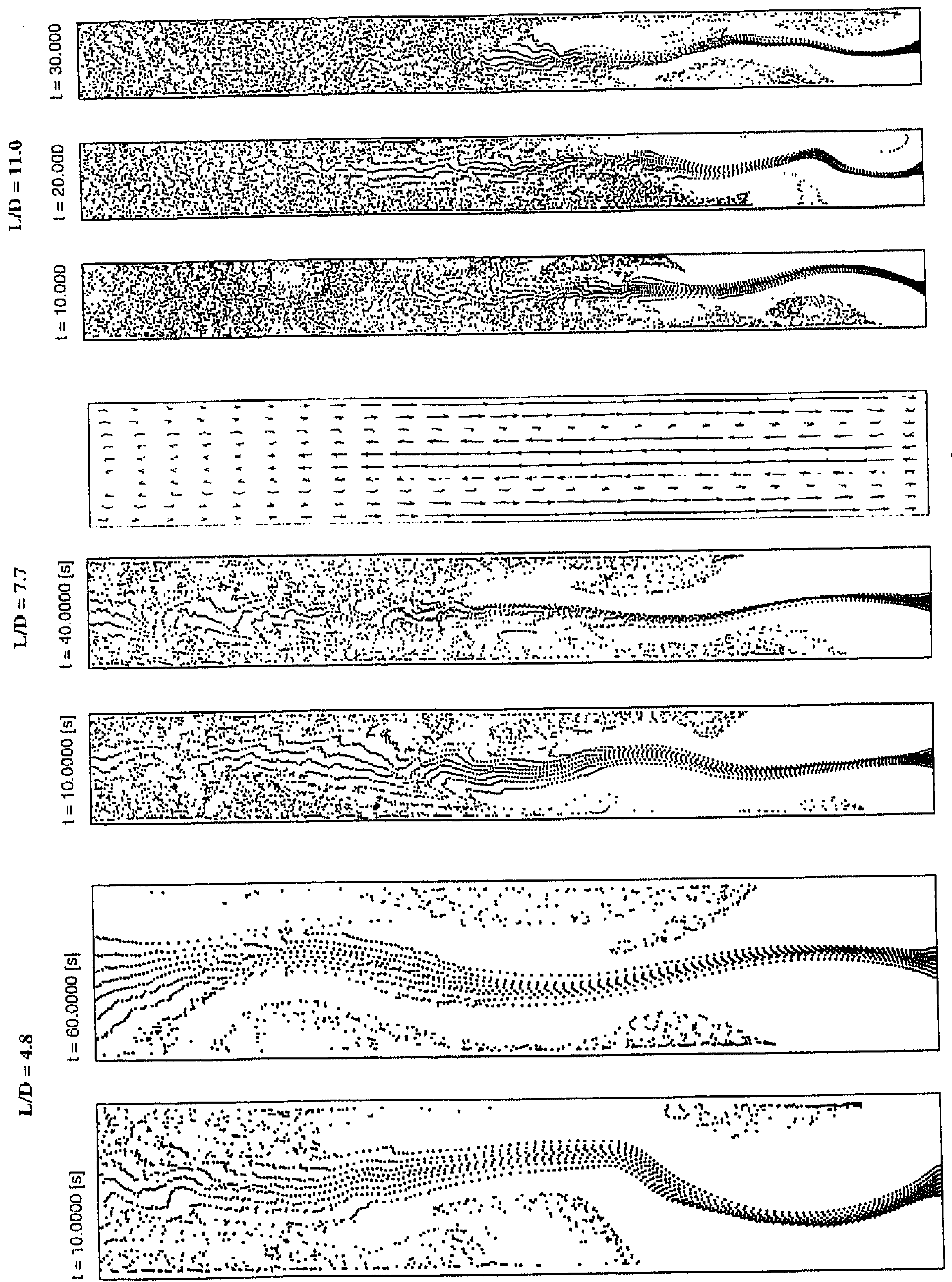
ber. For the purpose of reference in this figure the diagram presented by Grace (1973) and Grace et al. (1976) is included, which shows the effect of fluid properties and equivalent bubble diameter on the bubble characteristics (i.e., shape and terminal velocity). The relevant parameter values corresponding to the simulation results presented in Fig. 26 are listed in Table V. From Fig. 26 it can be seen that the bubble corresponding to case $d$ (the wobbling regime) exhibits an oscillatory motion during its rise through the liquid. From computer animations it could be clearly seen that this phenomenon is caused by vortices that are shed in an alternating mode at the left and right rear part of the bubble, similar to vortices that are shed behind a (stationary) circular cylinder subjected to cross-flow (Schlichting, 1975). As evident from inspection of Fig. 26 the computed bubble shapes show very close resemblance with those expected on the basis of the aforementioned diagram. It should be mentioned here that the diagram presented by Grace (1973) and Grace $e t$ al. (1976) is valid for three-dimensional gas bubbles rising in an unbounded Newtonian liquid, whereas the model is based on a two-dimensional approach where the bubbles rise in a system with finite lateral dimensions. These two differences were felt to be responsible for the fact that the bubble rise velocities inferred from this diagram systematically exceeded the computed bubble rise velocity found by Delnoij et al. Therefore they also studied the effect of the column width on the magnitude of bubble rise velocity and found an increasing bubble rise velocity with increasing column width at fixed (equivalent) bubble diameter. This is expected behavior because in a system with relatively small lateral dimension there is less space for the downward-moving liquid induced by the rising gas bubble.

In addition Delnoij et al. used the VOF method to compute the coalescence of two coaxial gas bubbles of identical size generated at the same orifice. Figure $27 \mathrm{a}$ shows the temporal evolution of the positions of the two gas bubbles. From the sequence of bubble positions it can be seen that the trailing bubble moves faster than the leading bubble and eventually at $t=0.42 \mathrm{~s}$ coalescence of the two gas bubbles commences. From computer animations it could clearly be seen that just after completion of the coalescence process (at $t=0.45 \mathrm{~s}$ ) a "splashing" liquid

TABLE V

VAlUES OF THE EOTVÖS (EO) AND MORTON (M) Numbers USEl) IN THE Simulations Depicted IN Fig. 6.11

\begin{tabular}{llrlll}
\hline Calse & Regime & Eo & \multicolumn{1}{c}{$\mathrm{M}$} & $\begin{array}{c}D^{\prime \prime} \\
(\mathrm{m})\end{array}$ & $\begin{array}{c}L^{\prime \prime} \\
(\mathrm{m})\end{array}$ \\
\hline $\mathrm{a}$ & Spherical & 1 & $1.4 .10^{-4}$ & 0.01 & 0.02 \\
$\mathrm{~b}$ & Ellipsoidal & 10 & 0.137 & 0.05 & 0.10 \\
$\mathrm{c}$ & Spherical cap & 100 & $2.5 .10^{-11}$ & 0.10 & 0.20 \\
$\mathrm{~d}$ & Wobbling & 2 & $2.5 .10^{-11}$ & 0.02 & 0.06 \\
\hline
\end{tabular}

" $D$ and $L$. respectively. represent the lateral and vertical dimensions of the computational domain. 
(d)

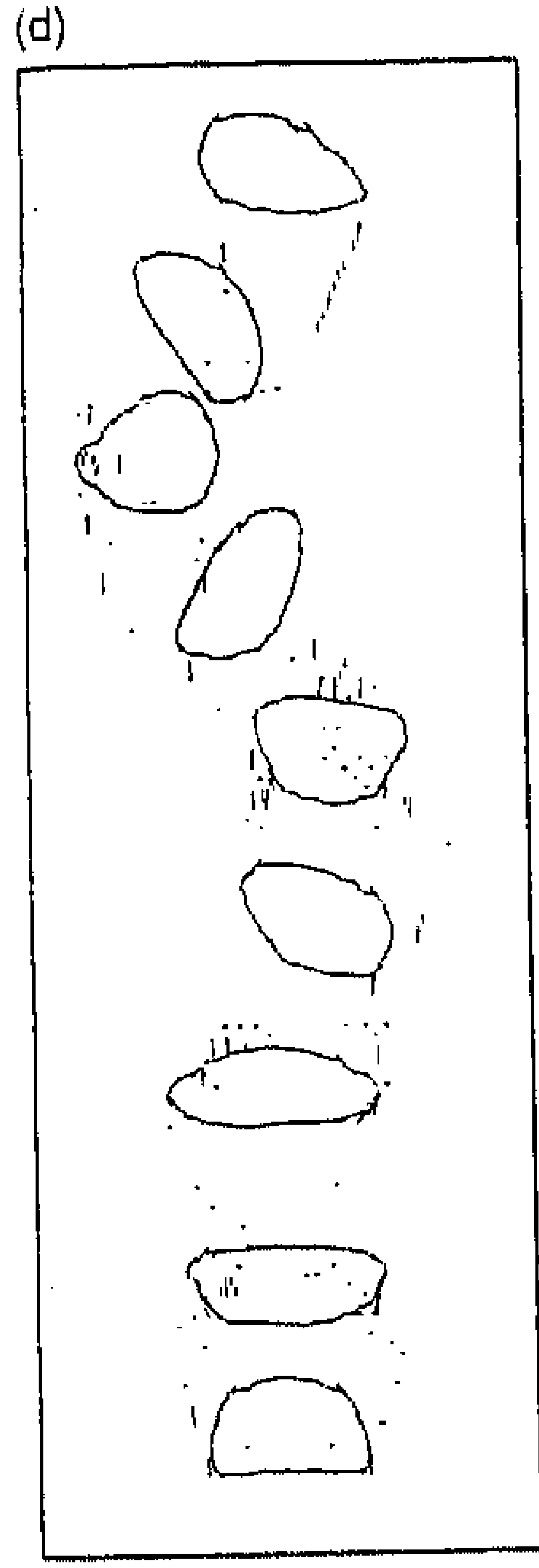

(c)

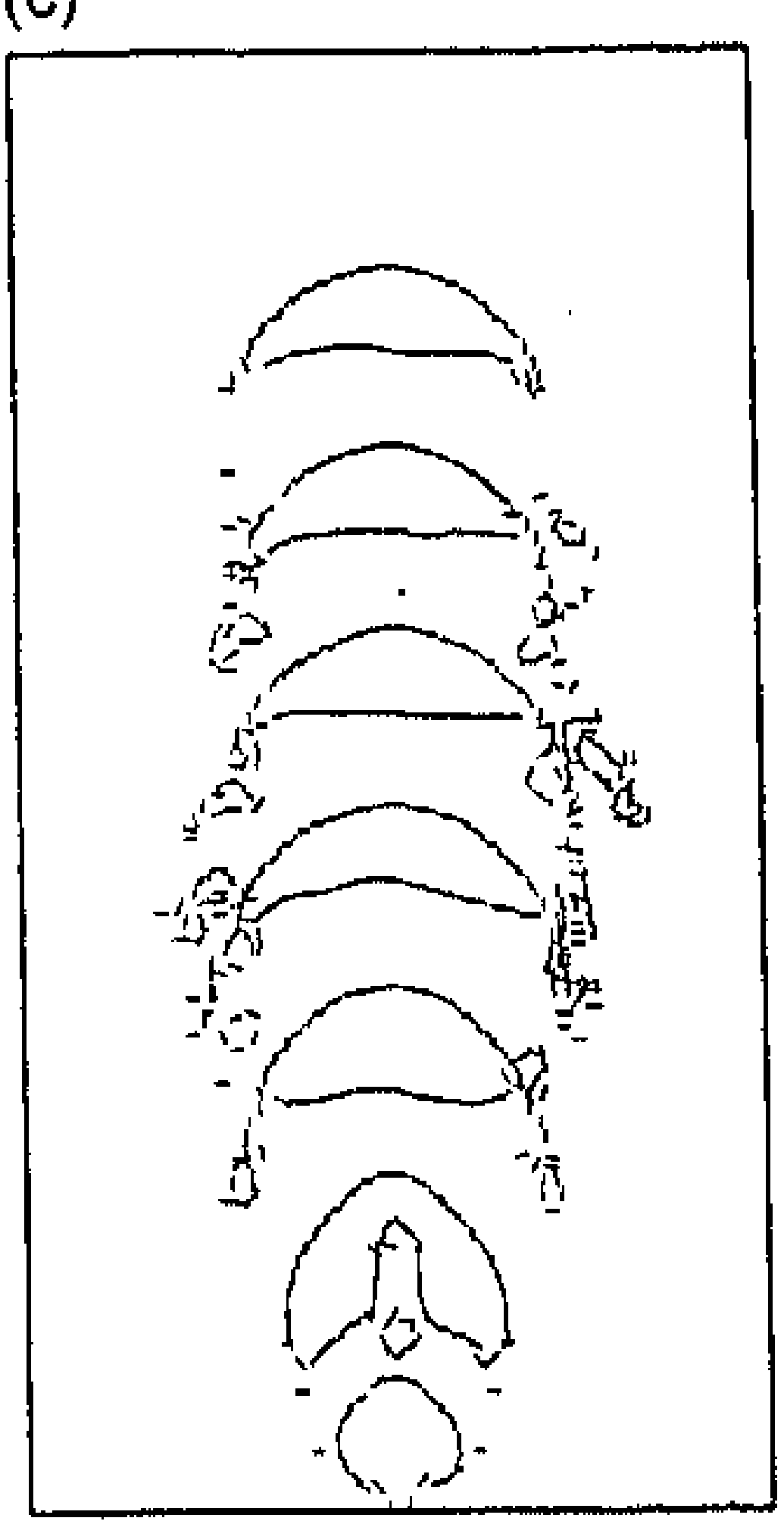

(b)

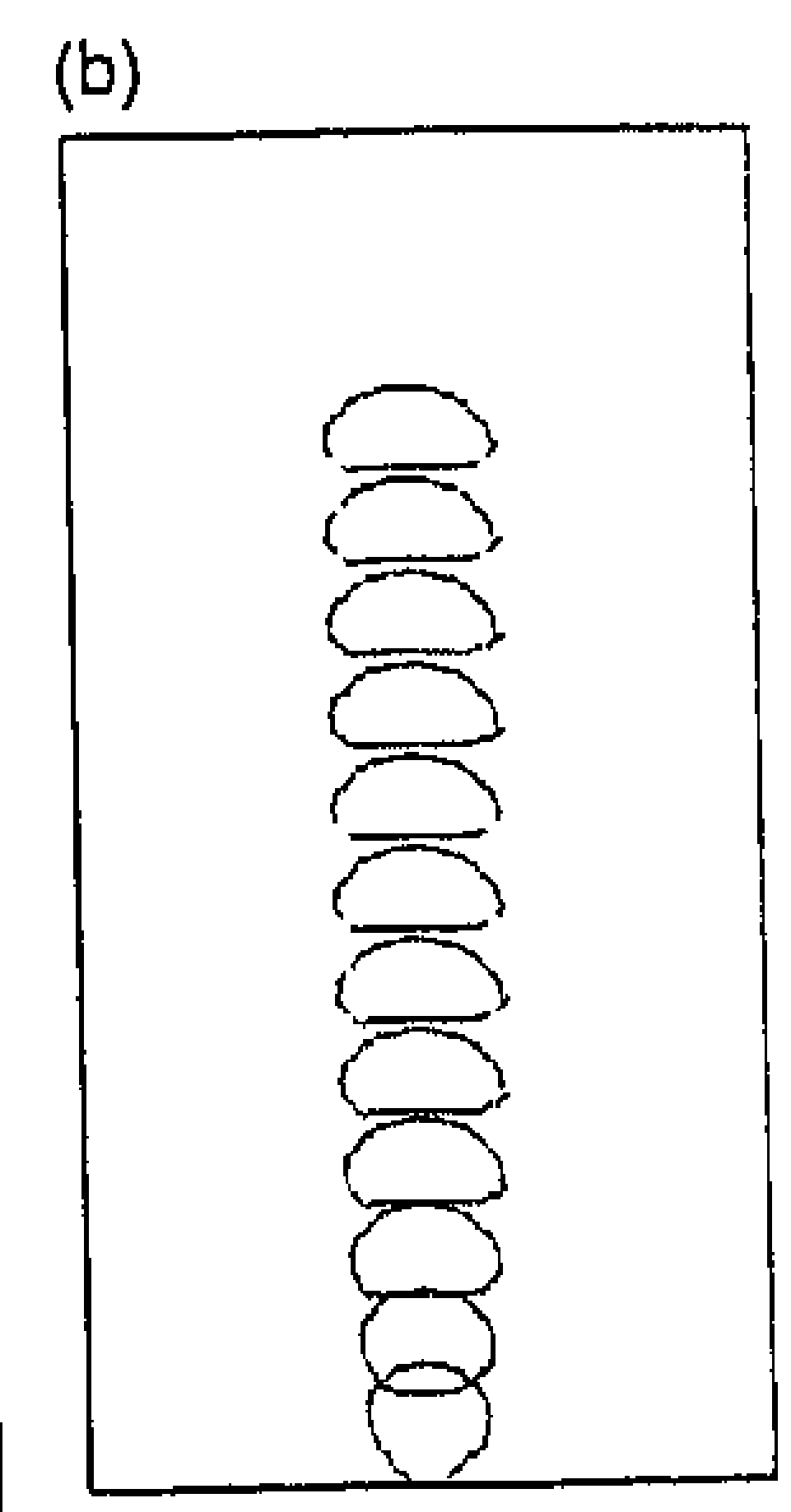

(a)

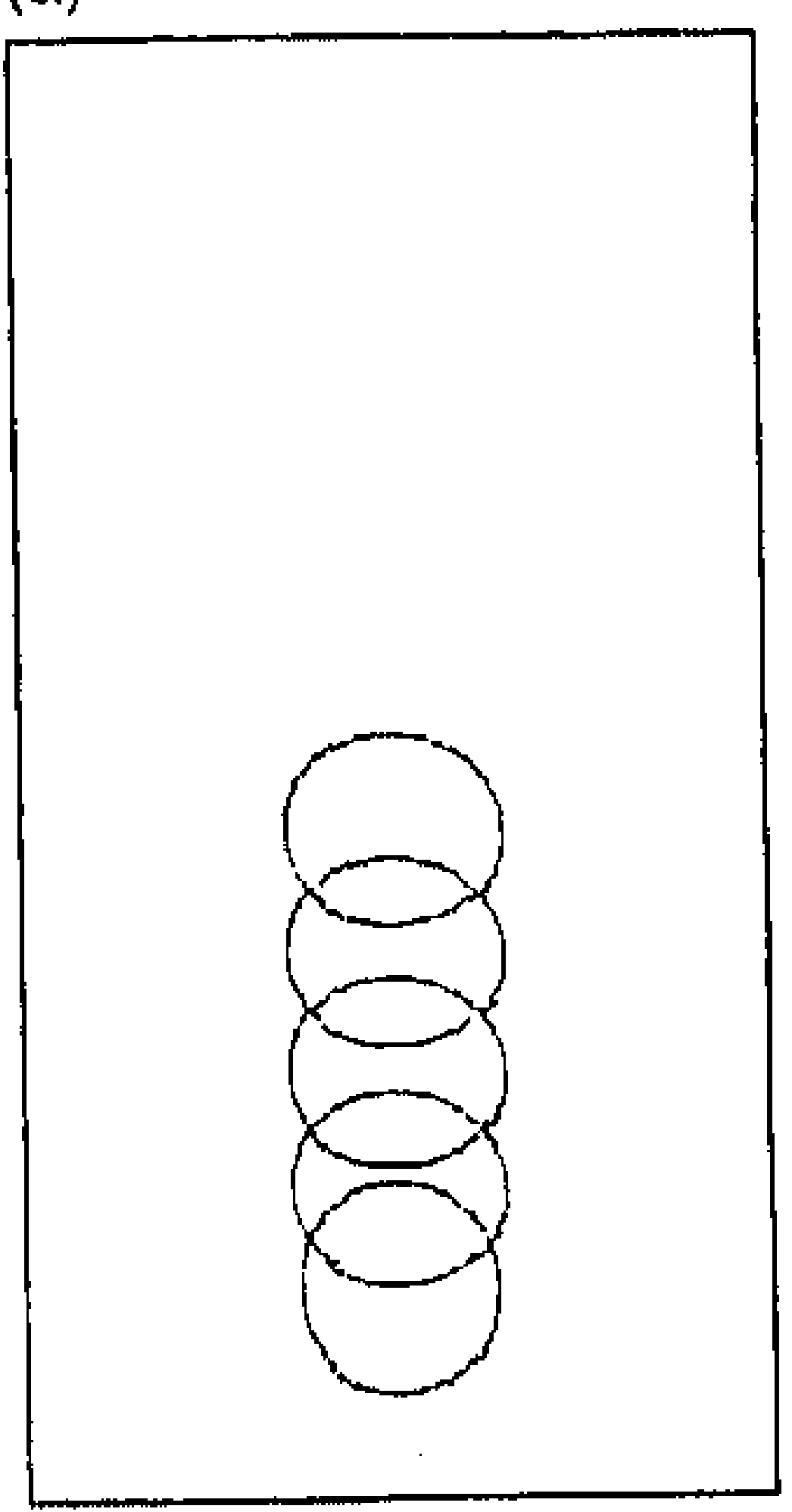

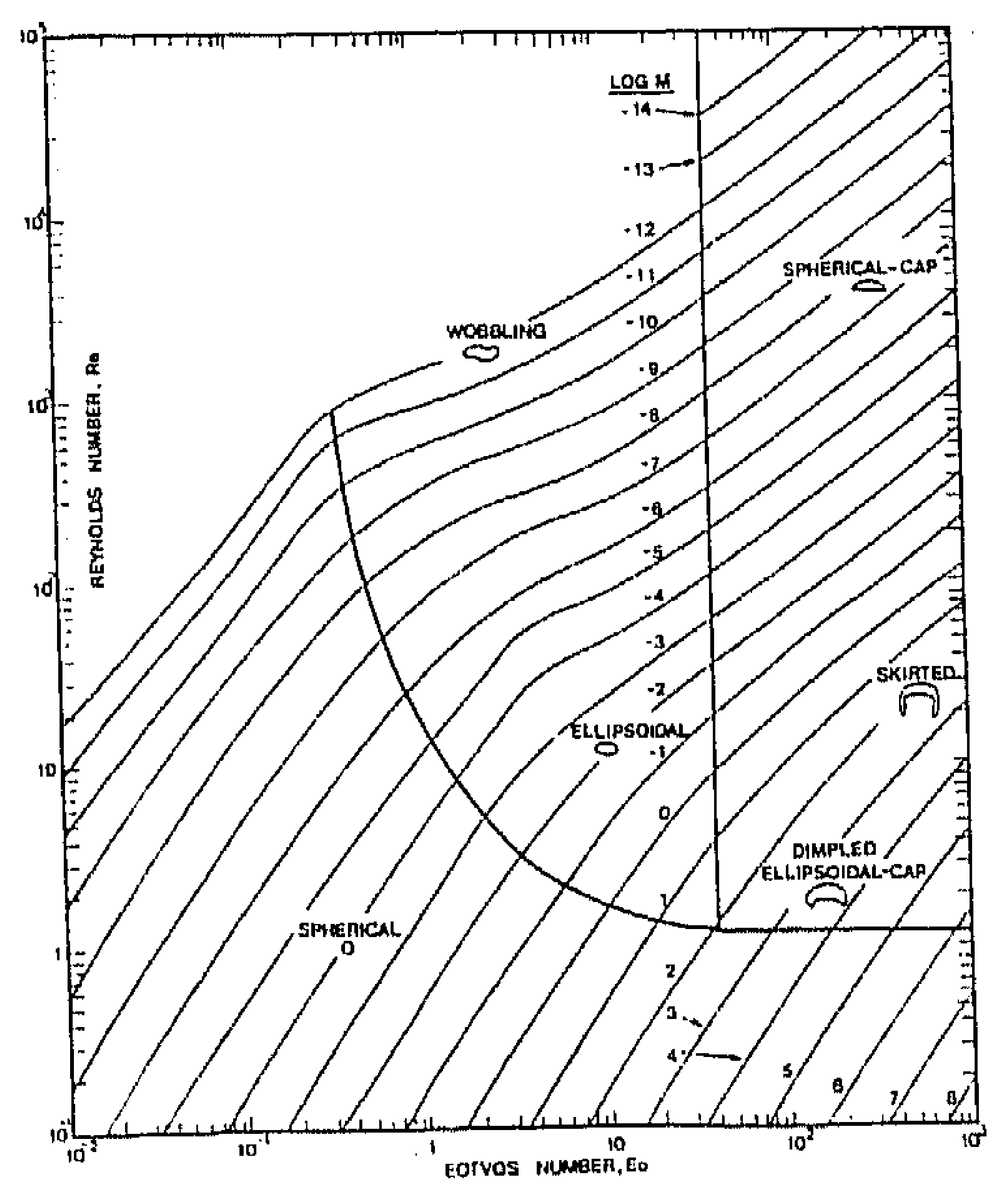

FIG. 26. Computed formation and rise of single gas bubbles emanating from a central orifice for top) various values of the Eotvös (Eo) and Morton $(\mathrm{M})$ number and (bottom) graphical correlation due to Grace (1973) and Grace et al. (1976), 
(a)

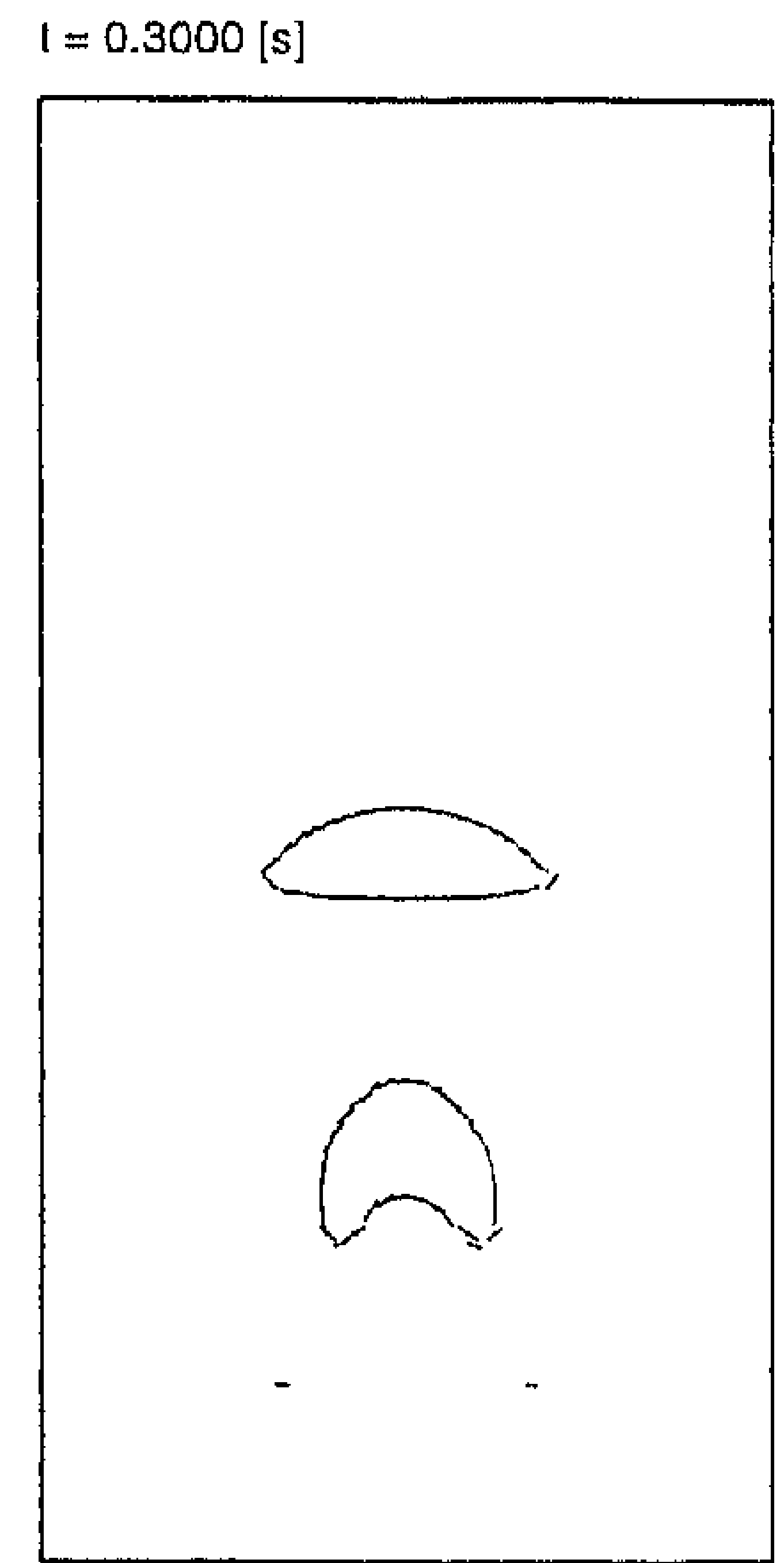

$t=0.3900[s]$

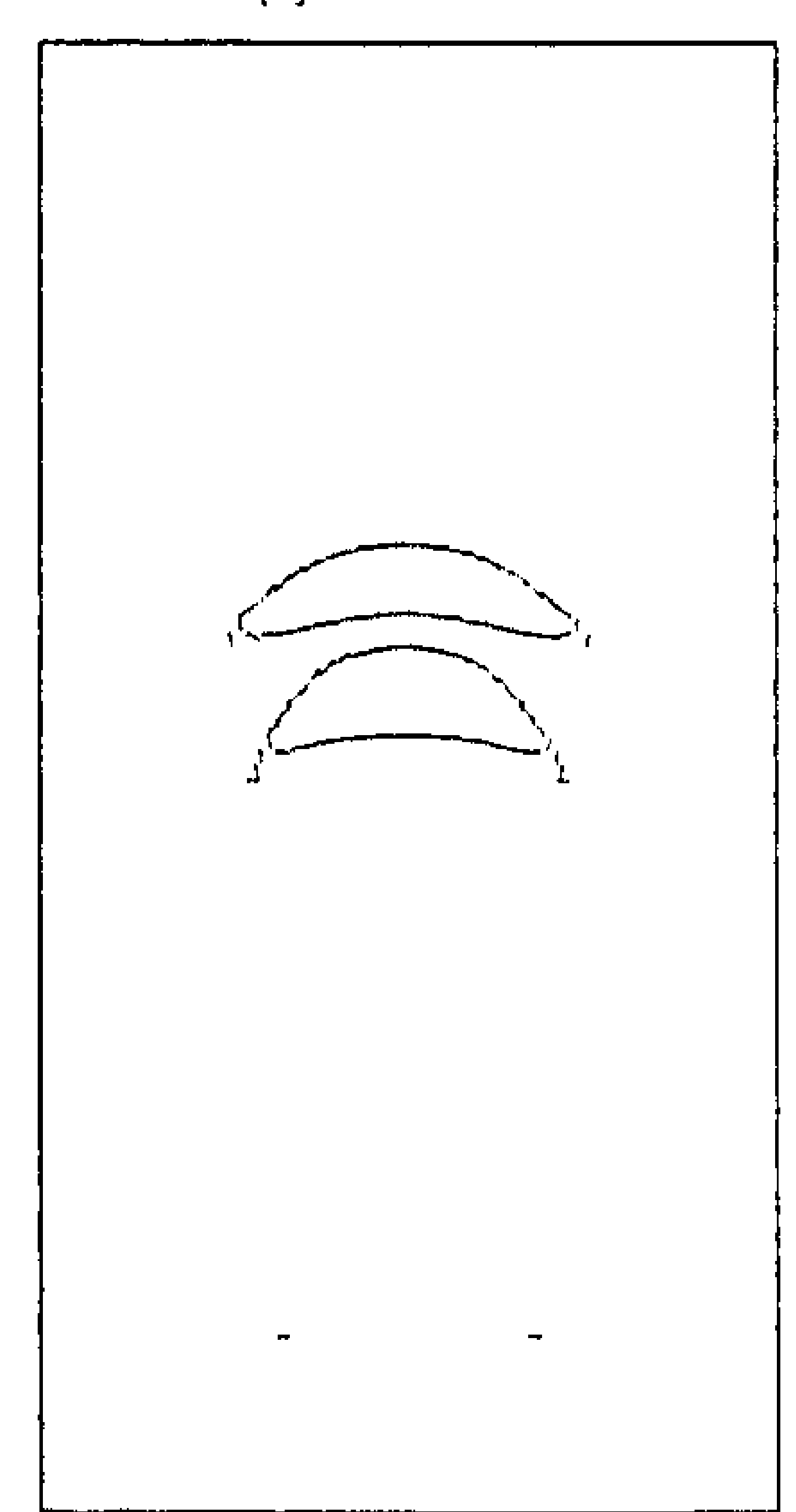

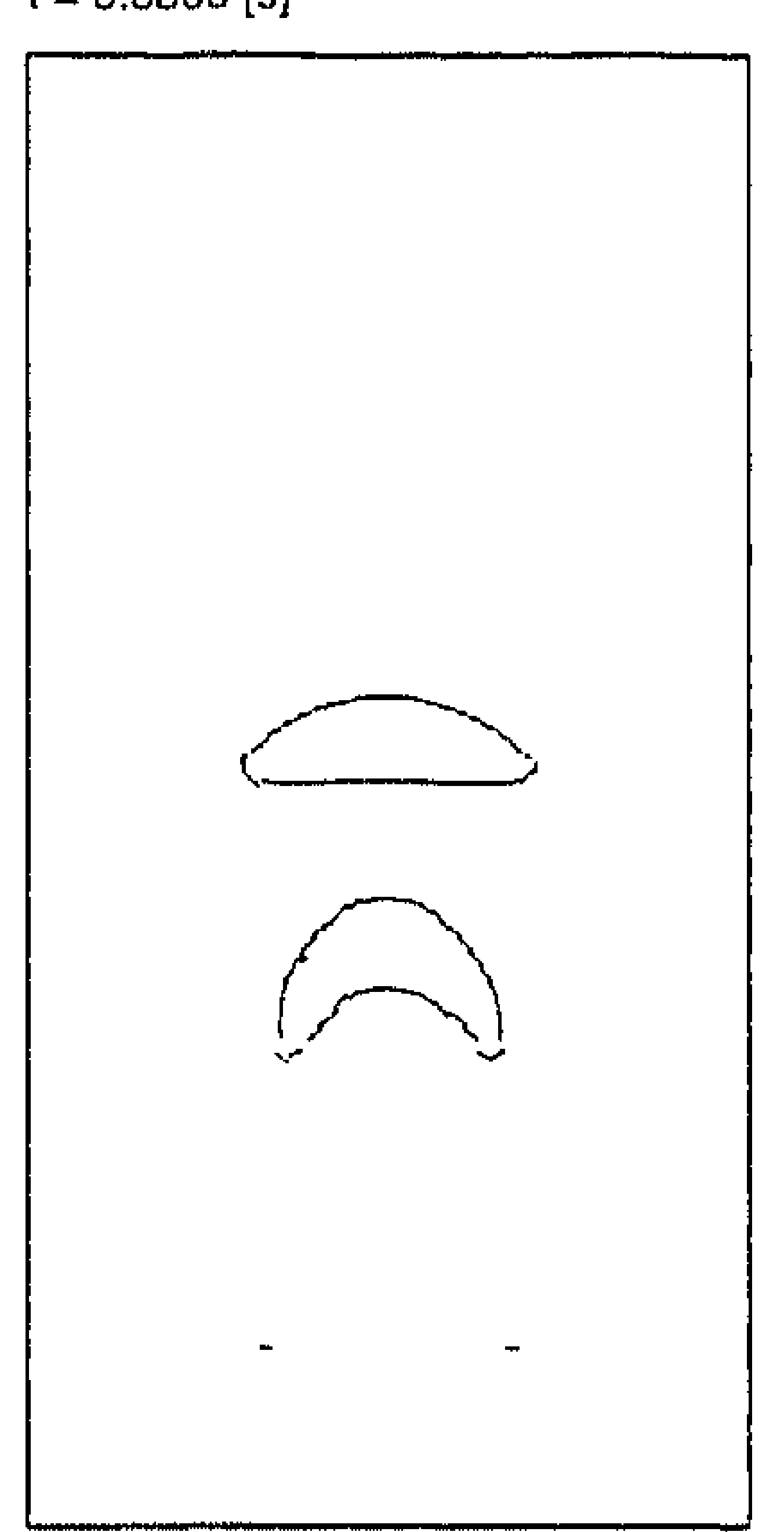

$t=0.4200[s]$

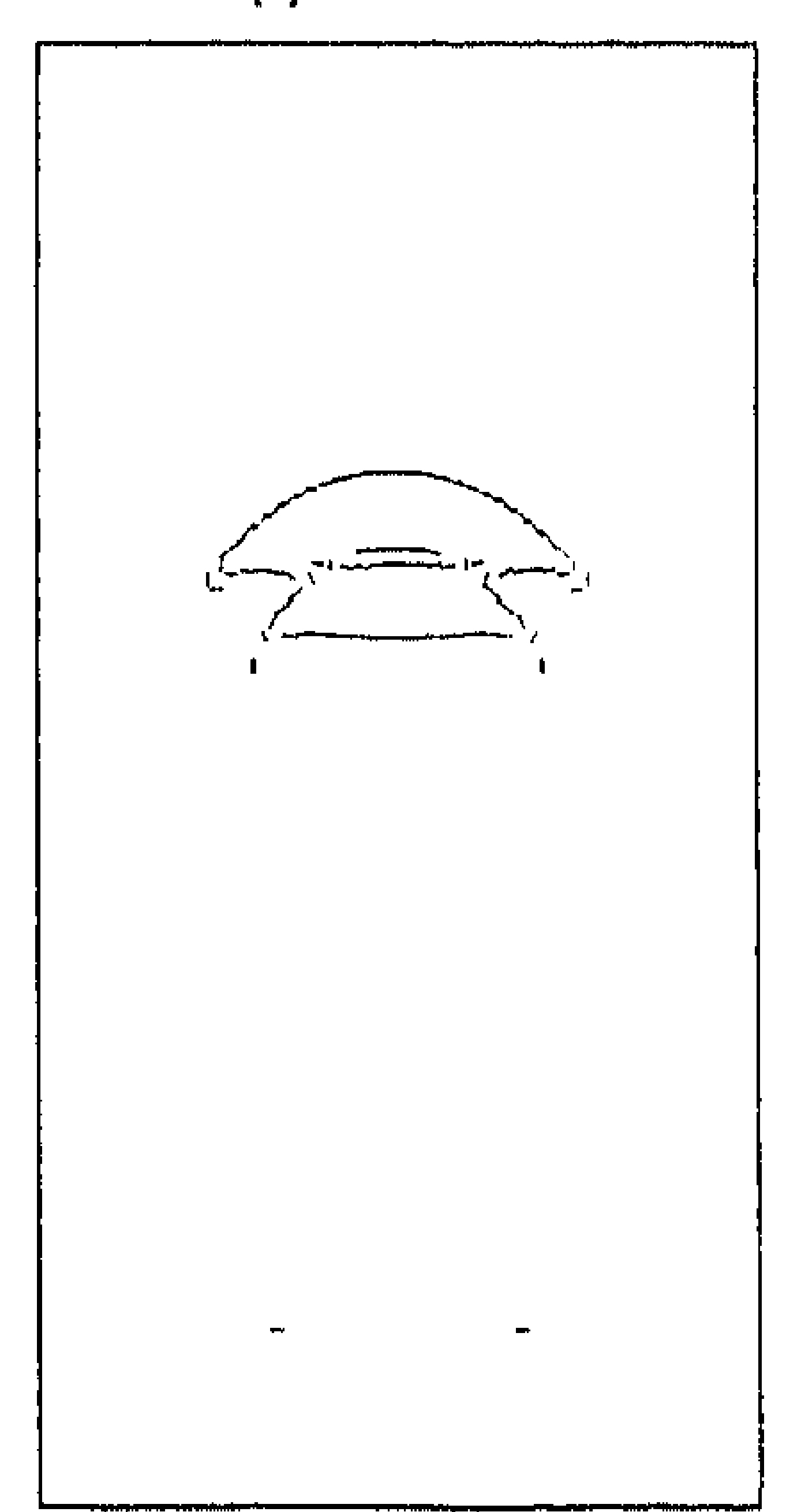

$t=0.3600[s]$

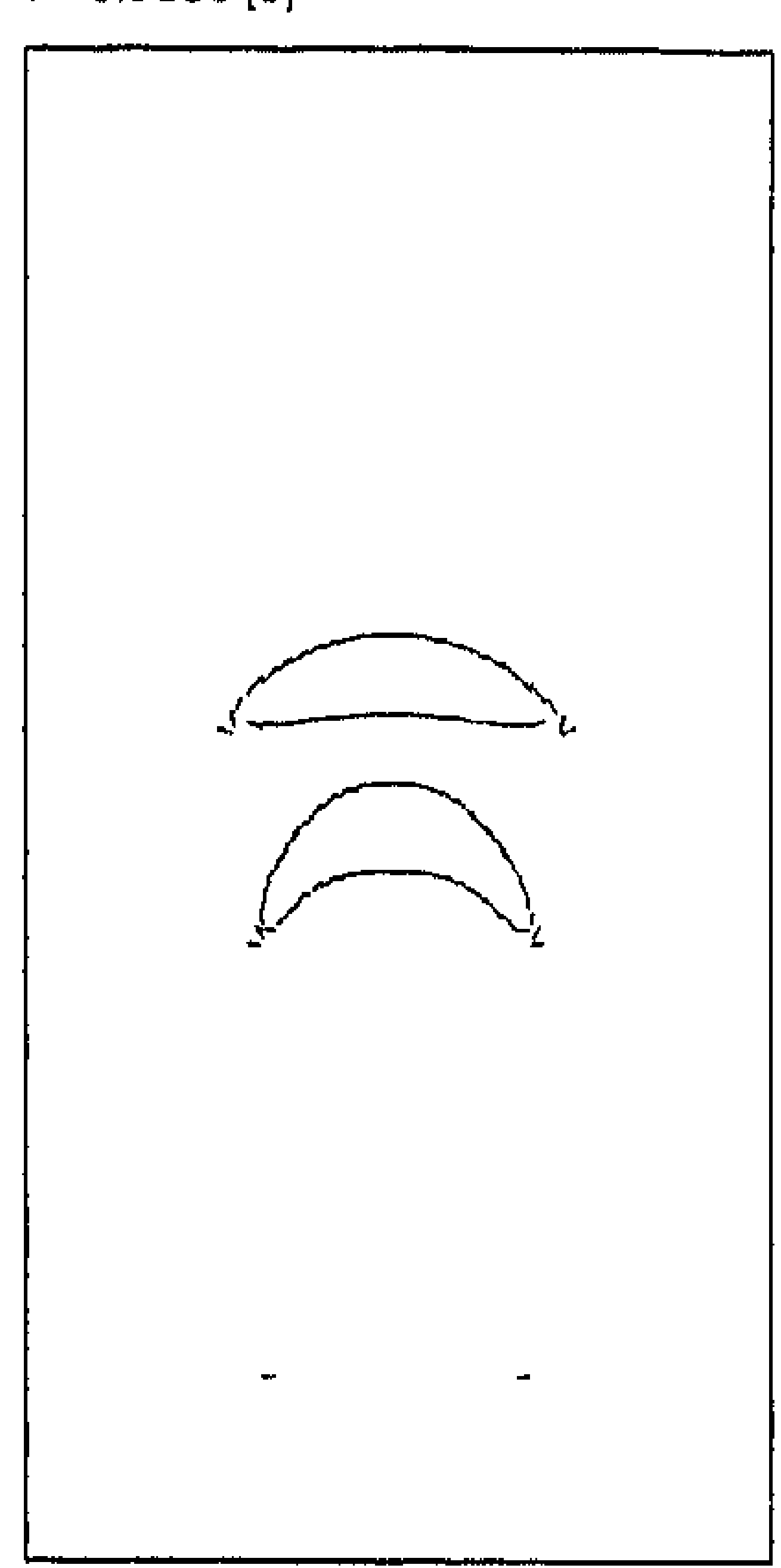

$t=0.4500[s]$

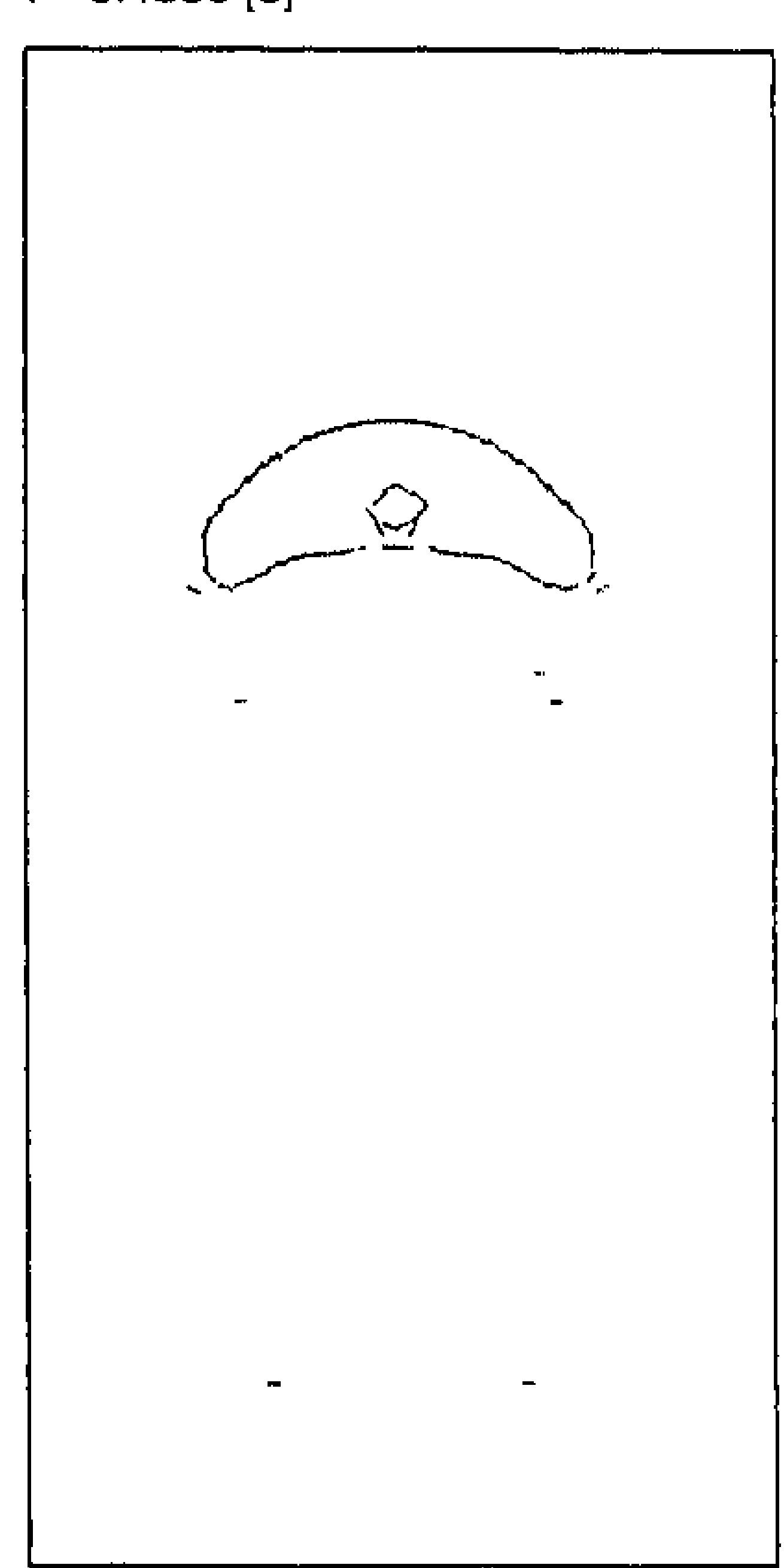

FIG. 27. (a) Experimentally observed and (b) theoretically predicted coalescence between two coaxial bubbles generated at an orifice $\left(E_{0}=16, \mathrm{M}=2.0 .10^{-4}\right.$, lateral $(D)$ and vertical $(L)$ dimensions of the computational domain: $D=0.05 \mathrm{~m}, L=0.10 \mathrm{~m}$ ). 
(b)

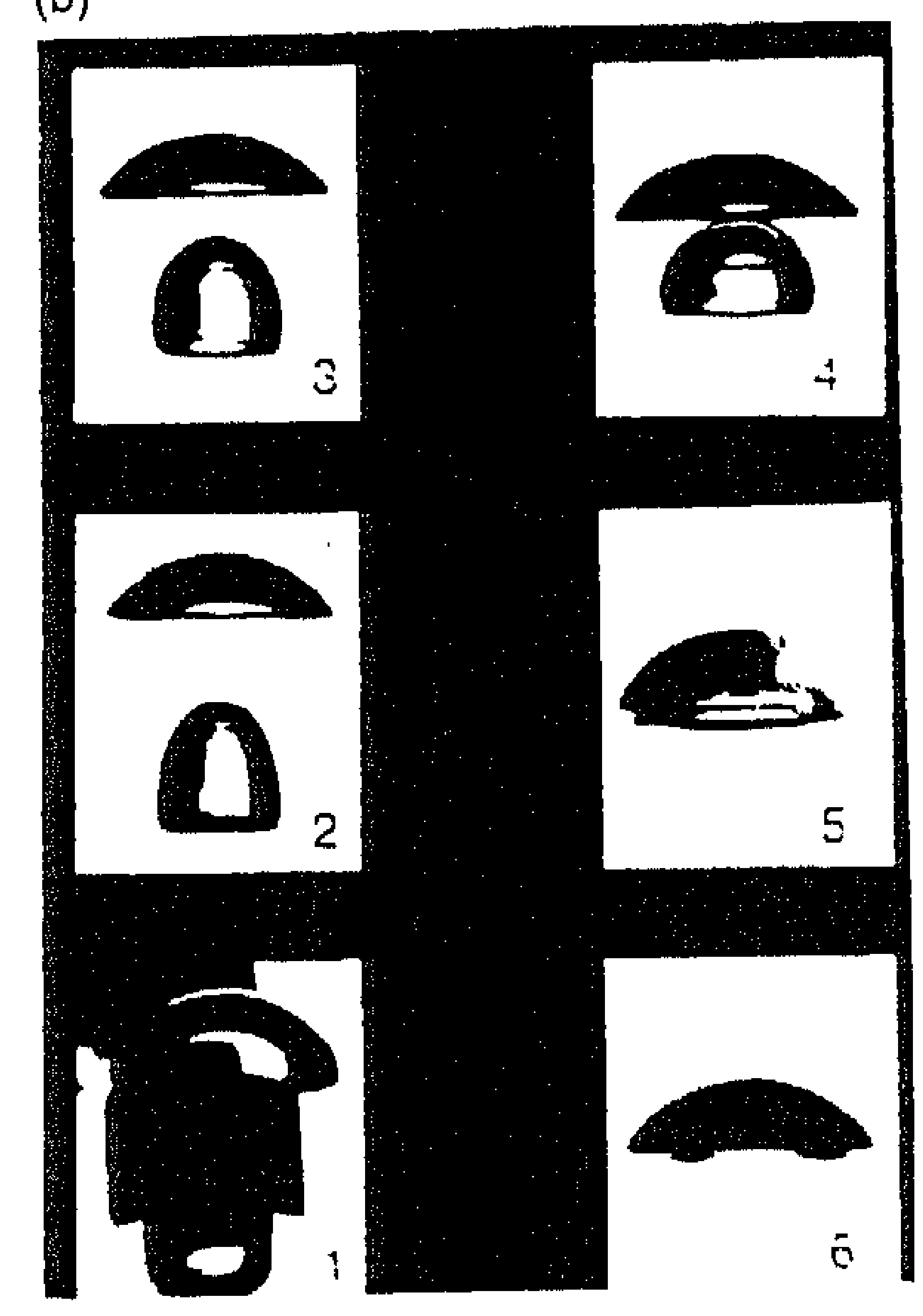

FIG. 27. b, Continued

pocket forms at the bubble base. Due to the fact that the trailing bubble rises in the wake region of the leading bubble, a difference in bubble shape develops. In Figure $27 \mathrm{~b}$ the results of the corresponding experiment, reported by Brereton and Korotney (1991), are shown. From a comparison of the theoretical and experimental results one can conclude that a reasonable agreement is obtained, especially in view of the complexity of the coalescence process. Moreover, one should keep in mind that no adjustable parameters were used in the VOF model. Finally Fig. 28 shows the positions of the two coaxial gas bubbles at $t=0.33 \mathrm{~s}$ together with the distribution of the relative velocity field of the liquid phase. In this figure the instantaneous velocity of the leading gas bubble was subtracted from the instantaneous liquid phase velocity distribution. From Fig. 28 it can be seen that at $t=0.33 \mathrm{~s}$ the trailing bubble rises in the wake region of the leading bubble with upward (instead of downward) flowing liquid near its nose. As a consequence of this phenomenon, the trailing bubble attains a higher rise velocity than the leading bubble with bubble coalescence as the final result.

\section{E. Modeling of a Laminar Entrained Flow Reactor}

Within the context of a study aimed at the determination of the pyrolysis kinetics of polymers using a laminar entrained flow reactor (LEFR), Westerhout et al. (1996) developed a comprehensive model that couples a single-particle con- 


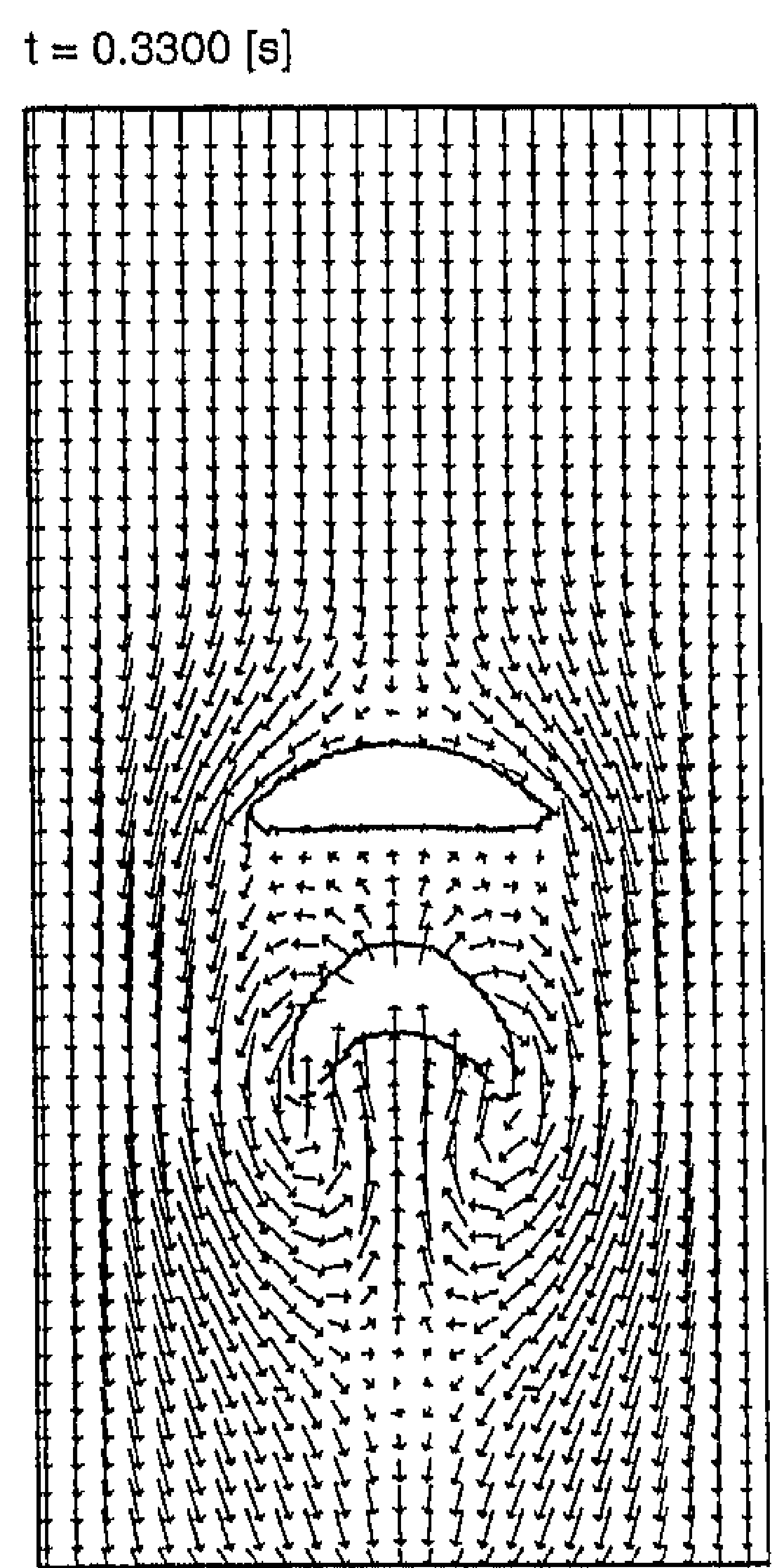

FlG. 28. Positions of two coaxial gas bubbles generated subsequently at the same orifice together with the distribution of the relative (i.e., with respect to the velocity of the leading gas bubble) liquid phase velocity.

version model with a CFD model describing the prevailing velocity and temperature distributions inside the reactor. The main characteristics of the reactor model developed by Westerhout et al. are as follows:

- The conversion of the polymer particles obeys first-order kinetics.

- The finite rate of heat penetration in the spherical polymer particles is accounted for.

- Due to the very low particle loading one-way coupling between the solid phase (i.e., the polymer particles) and the gas phase (nitrogen) prevails.

- The residence time of the polymer particles in the reactor and the temperature history experienced by these particles during their journey through the reactor is governed by the compressible nonisothermal Navier-Stokes equations.

- The gas phase flowing through the reactor is transparent with respect to thermal radiation.

In Fig. 29 a schematic drawing of the LEFR used by Westerhout et al. is shown. The polymer particles are fed to the reactor through a central cooled pipe ("cold finger") with the aid of a small nitrogen stream and subsequently they are heated by the annular nitrogen stream supplied through the flow distributor. Due to the 


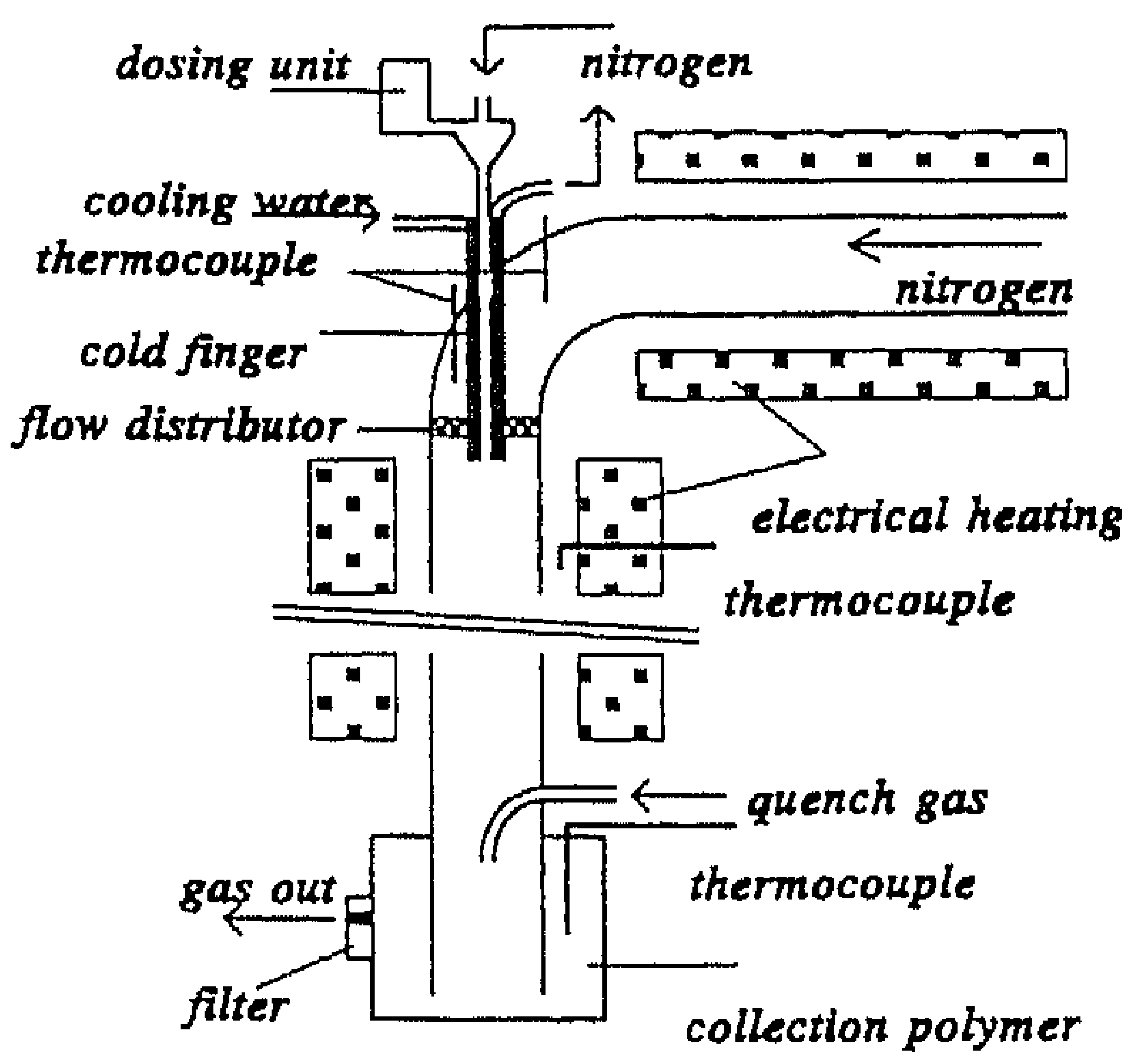

FIG. 29. Schematic drawing of the LEFR used by Westerhout et al. (1996).

velocity and temperature differences that exist between the central nitrogen stream (containing the polymer particles) and the annular nitrogen stream, the polymer particles experience a nonuniform (axial) velocity and temperature history which possibly influences their chemical conversion.

In Fig. 30a the radial profiles of the axial component of the gas velocity are shown at various axial locations, whereas the corresponding temperature profiles are shown in Fig. 30b. From these figures it can be seen that significant radial and axial velocity and temperature differences exist in the LEFR. The consequences of these phenomena for the chemical conversion of the polymer particles are depicted in Fig. 31. In this figure the computed conversion of the polymer particles is shown as a function of the axial coordinate for a case where the aforementioned velocity and temperature gradients are neglected and another case where they are taken into account. As evident from inspection of Fig. 31, significant differences exist between these two cases. By using the information on the velocity and temperature profiles prevailing in the LEFR it is possible to correct for these nonuniformities. This application demonstrates that CFD can also be used as a tool to interpret experimental data correctly.

\section{Conclusion}

a. Future Role of CFD in Chemical Reaction Engineering. From the review presented in this paper it should have become apparent that CFD offers great potential for the chemical engineer and that this rapidly emerging new hybrid science of mathematics and mechanics at present already has a profound impact on chemical reaction engineering. It is expected that the role of CFD in the future design of 
(a)

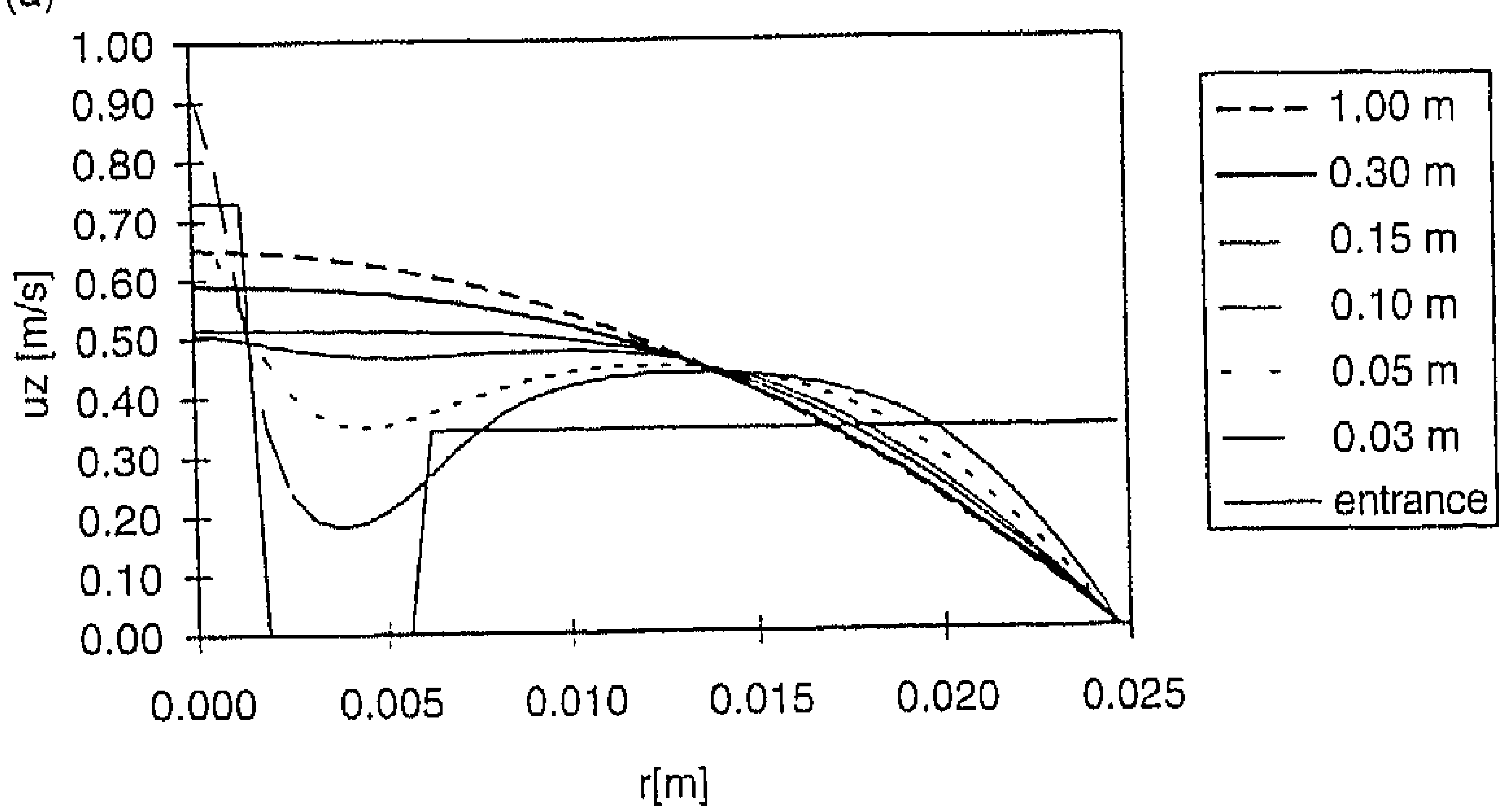

(b)

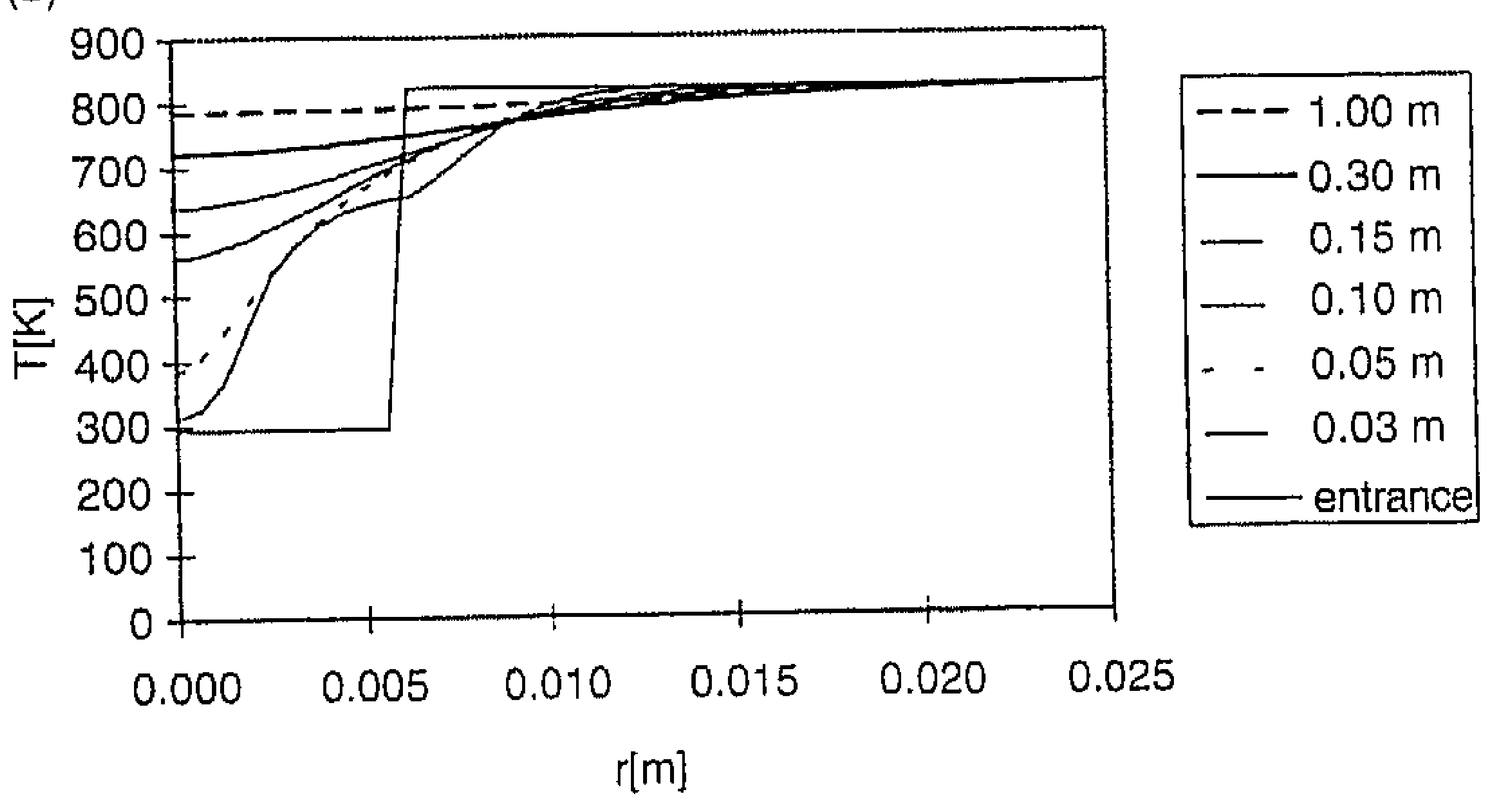

FIG. 30. (a) Computed radial profiles of the axial component of the gas velocity at various axial locations and (b) corresponding temperature profiles for LEFR studied by Westerhout et al. (1996).

chemical reactors will increase substantially and that CFD will reduce the experimental effort required to develop industrial (multiphase) reactors. Due to the anticipated future role of CFD in chemical reaction engineering the authors believe that the incorporation of CFD courses in chemical engineering curricula is of crucial importance. These courses should provide the chemical engineer at least with upto-date knowledge on the foundations, possibilities, and limitations of CFD in the context of chemical reaction engineering.

b. Directions for Future Research. The variety and degree of complexity of systems encountered in industrial practice demands an integrated modeling approach where models with an increasing degree of sophistication should be used to feed models that invoke submodels with a strong empirical base. As far as turbulent flows are concerned, in this respect both LES and DNS offer the possibility to gener- 


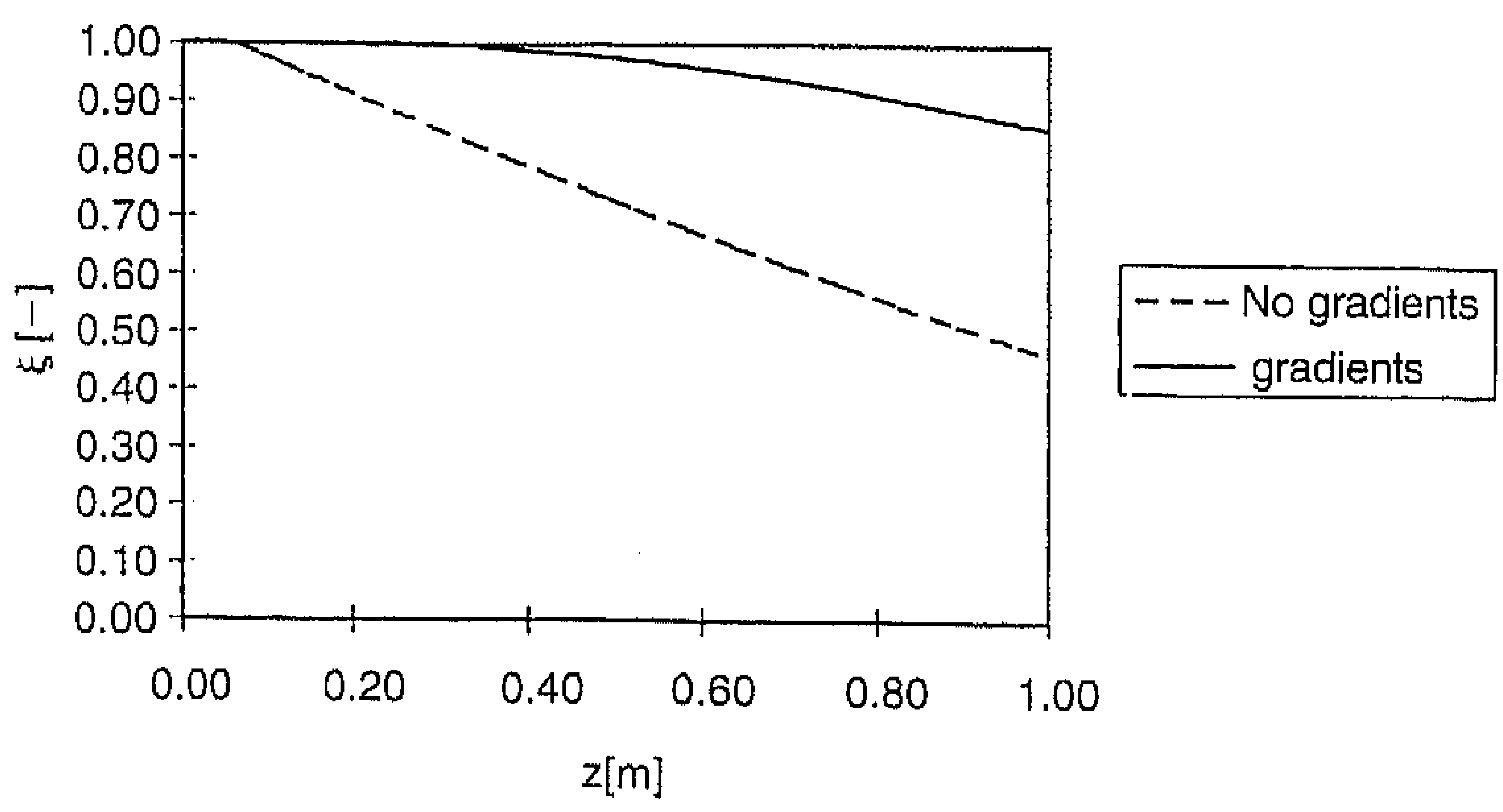

FIG. 31. Computed conversion of the polymer particle as a function of the axial coordinate for cases with and without accounting for existing velocity and temperature gradients in the LEFR.

ate databases to test and develop phenomenological turbulence models, whereas for dispersed multiphase flows mixed Eulerian-Lagrangian models offer the possibility to develop closure laws that can be used in a multifluid framework suited for the simulation of macroscopic systems of interest. In this connection Stokesian dynamics should also be mentioned because it offers great potential for simulation of concentrated suspensions in which hydrodynamic interaction has to be accounted for.

Non-Newtonian flows need much more attention since in many industrial applications the chemical engineer has to deal with materials that exhibit a complex rheological behavior that cannot (even approximately) be described with Newtonian closure models.

As is evident from inspection of Table III turbulence modeling of multiphase flow systems requires major attention in the near future. Also the development of closure laws for phenomena taking place in the vicinity of interfaces such as coalescence, breakup, and accumulation of impurities should be considered in more detail. Once these requirements have been met, in principle, it would be possible to predict a.o. flow regime transition and the spatial distribution of the phases with confidence, which is of utmost importance to the chemical engineer dealing with the design of (novel) multiphase reactors.

c. Experimental Validation. As mentioned earlier, at the present state of theoretical development carefull experimental validation of all CFD predictions involving turbulent (multiphase) flows in complex geometries is of crucial importance. Due to this necessity there exists, especially for multiphase flow systems, a strong demand for the further development of nonintrusive experimental techniques that permit measurement of both the time-average and the fluctuating component of key quantities such as phase volume fractions, phase velocities, and species concentration. The 
requirement for the measurement of fluctuating components of key quantities is due to the fact that multiphase flows very often exhibit oscillatory behavior.

d. Commercial CFD Packages. At present a large number of commercial CFD packages are available, each with their own specific areas of application. In the near future the role of these packages in both academic and industrial research applications will most likely expand. At the same time it appears that the most significant efforts of the vendors of commercial CFD packages are directed toward the development of more advanced preprocessing and postprocessing facilities including a.o. grid generation and visualization of the computational data and not toward the development of new and more detailed physical models, an activity which is apparently left to the users of these packages. As far as this last activity is concerned. it is of crucial importance that the CFD package provide for an open programming environment where the user has full access to all relevant parts of the code so he or she can successfully implement and validate submodels.

\section{Nomenclature}

$\bar{a} \quad$ Acceleration. $\mathrm{m} / \mathrm{s}^{2}$

$C_{\mu} \quad$ Empirical constant

D diameter, $\mathrm{m}$

$\mathrm{Da} \quad$ Damköhler number

$D_{i} \quad$ Fickian diffusion coefficient of species $i, \mathrm{~m}^{2} / \mathrm{s}$

e Restitution coefficient

Internal energy per unit mass. $\mathrm{J} / \mathrm{kg}$

$e_{k} \quad$ Internal energy per unit mass for phase, $k, \mathrm{~J} / \mathrm{kg}$

$E \quad$ Differential residence time distribution function, $\mathrm{s}^{-1}$

$E_{k l} \quad$ Energy transfer rate between phase $k$ and $l$ per unit volume, $\mathrm{W} / \mathrm{m}^{3}$

Eo Eotvös number

$F \quad$ Cumulative residence time distribution function

Fractional amount of fluid

$\bar{F}_{i} \quad$ Force acting on $i$ th particle, $\mathrm{kg} \cdot \mathrm{m} / \mathrm{s}^{2}$

$\bar{g} \quad$ Gravitational force per unit mass, $\mathrm{m} / \mathrm{s}^{2}$

G $\quad$ Solids mass flux. $\mathrm{kg} /\left(\mathrm{m}^{2} \cdot \mathrm{s}\right)$

$h \quad$ Wall-to-bed heat transfer coefficient, $\mathrm{W} /\left(\mathrm{m}^{2} \cdot \mathrm{K}\right)$

I Identity tensor

$\bar{J}_{i} \quad$ Mass flux vector of species $i$ due to molecular transport, $\mathrm{kg} /\left(\mathrm{m}^{2} \cdot \mathrm{s}\right)$

$\bar{J}_{k, 1} \quad$ Mass flux vector of species $i$ in phase $k$ due to molecular transport, $\mathrm{kg} /\left(\mathrm{m}^{2} \cdot \mathrm{s}\right)$

$k \quad$ Turbulent kinetic energy, $\mathrm{m}^{2} / \mathrm{s}^{2}$

Thermal conductivity, $\mathrm{W} /(\mathrm{m} \cdot \mathrm{K})$ 


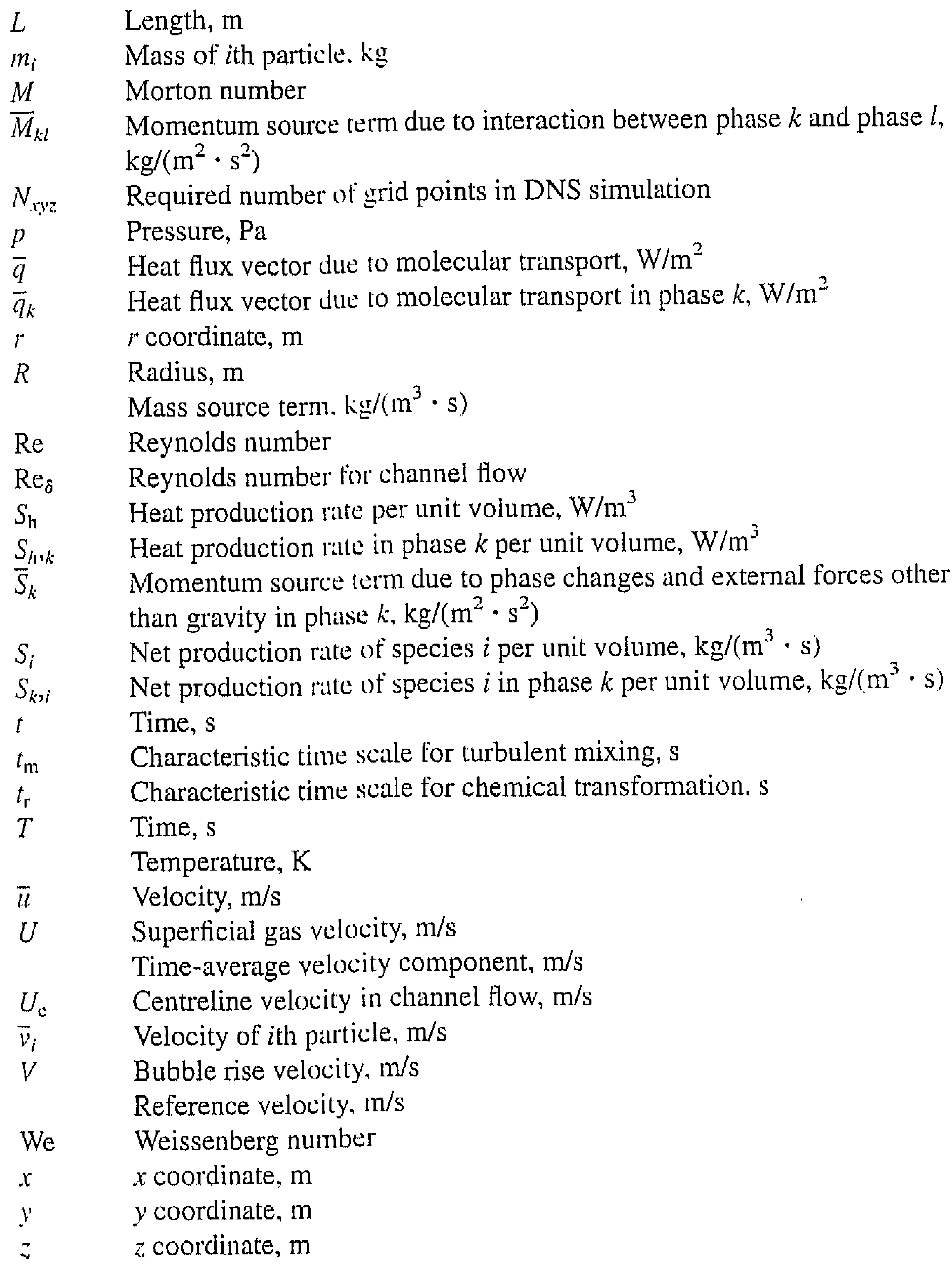

\section{Greek Letters}

$\delta \quad$ Channel half-width, $m$

$\epsilon \quad$ Volume fraction

Viscous dissipation rate, $\mathrm{m}^{2} / \mathrm{s}^{3}$ 


$\begin{array}{ll}\gamma & \begin{array}{l}\text { Bulk viscosity, } \mathrm{kg} /(\mathrm{m} \cdot \mathrm{s}) \\ \text { Relaxation time in viscoelastic flow, } \mathrm{s}\end{array} \\ & \begin{array}{l}\text { Linear dimension of smallest eddies in turbulent flow, } \mathrm{m} \\ \text { Shear viscosity, } \mathrm{kg} /(\mathrm{m} \cdot \mathrm{s})\end{array} \\ & \begin{array}{l}\text { Dynamic friction coefficient } \\ \nu\end{array} \quad \text { Kinematic viscosity, } \mathrm{m}^{2} / \mathrm{s} \\ \omega_{i} & \text { Mass fraction of species } i \\ \omega_{k . i} & \text { Mass fraction of species } i \text { in phase } k \\ \rho & \text { Density, } \mathrm{kg} / \mathrm{m}^{3} \\ \sigma & \text { Surface tension, Pa } \cdot \mathrm{m} \\ \tau & \text { Stress tensor. Pa } \\ \tau_{\mathrm{p}} & \text { Particle response time. } \mathrm{s} \\ \tau_{\mathrm{k}} & \text { Kolmogoror time scale. } \mathrm{s} \\ \xi & \text { Chemical conversion }\end{array}$

Subscripts

b Bed

Bubble

c Centerline

h Heat

i Particle index number Species index

$k \quad k$ th phase in multiphase system

$\mathrm{K}$ Kolmogorov

$\mathrm{m} \quad$ Turbulent mixing

p Particle

$r$ Chemical transformation

s Solids

$t$ Terminal

w Wall

$z \quad$ Axiál direction

\section{Superscripts}

$\begin{array}{ll}\mathrm{t} & \text { Turbulent } \\ \mathrm{T} & \text { Transpose } \\ \text {, } & \text { Vector quantity } \\ \text { - } & \text { Fluctuating component } \\ & \text { Time average }\end{array}$




\section{Operator}

$\begin{array}{ll}\nabla & \text { Gradient } \\ \nabla \cdot & \text { Divergence } \\ <> & \text { Average }\end{array}$

\section{References}

Abid, M., Xuereb, C., and Bertrand. J., Hydrodynamics in vessels stirred with anchors and gare agitators: Necessity of 3-D modeling. Trans. I. Chem. E. 70(Part A), 377 (1992).

Adrian, R. J., Particle-imaging techniques for experimental fluid mechanics. Annu. Rev. Fluid Mech. 23, 261, (1991).

Alamdari, F., Edwards, S. C., and Hammond, S. P., Microclimate pertormance of an open atrium oftice building: A case study in thermo-fluid modeling. In "Computational Fluid Dynamics tor the Environmental and Building Services Engineer-Tool or Toy?", The Institution of Mechanical Engineers, London, 1991, p. 81.

Amsden, A. A., "KIVA II: A Computer Program for Chemically Reactive Flows with Sprays." U.S Department of Commerce NTIS Report LA-11560-MS, May 1989.

Amsden, A. A., "KIVA: A Computer Program for Two- and Three-Dimensional Fluid Flows with Chemical Reactions and Fuel Sprays," U.S. Department of Energy Report LA-10245-MS/UC32 and UC-34, Feb. 1985.

Amsden, A. A., and Harlow, F. H., "The SMAC Method: A Numerical Technique for Calculating Incompressible Fluid Flows," Los Alamos Scientific Laboratory Report LA-4370, 1970.

Anagnostopoulos, P., and Iliadis, G., Numerical study of the blockage effect on viscous flow past a circular cylinder. Int. J. Num. Methods Fluids 22, 1061 (1996).

Anderson, J. D., "Computational Fluid Dynamics: The Basics with Applications," McGraw-Hill. New York, 1995.

André, C., David, R., André, J., and Villermaux, J., A new fluorescence method for measuring crossfluctuations of two non-reative components in a mixing device. Chem. Eng. Technol. 15, 182 (1992).

Angelov, G., Journe, E., and Gourdon. C., Simulation of the flow patterns in a disc and doughnut column. Chem. Eng. J. 45, 87 (1990)).

Bader, R., Findlay, J., and Knowlton, T., Gas/solids flow patterns in a $30.5 \mathrm{~cm}$ diameter circulating bed. In "Circulating Fluidized Bed Technology II" (P. Basu, ind J. F. Large, eds.). Pergamon Press, New York, 1988, p. 123.

Bai, X. S., and Fuchs, L., Numerical model for turbulent diffusion flames with applications. In "Computational Fluid Dynamics" (C. Hirsch, J. Periaux and W. Kordullit, eds.). Elsevier, Amsterdam. 1992, vol. 1, p. 169

Baker, A. J., "Finite Element Computational Fluid Mechanics," Hemisphere Publishing Corporation, New York, 1985

Bakker, A., and van den Akker, H. E. A., A computational model for the gas-liquid flow in stirred reactors. Truns. I. Chem. E. 72(Part A), 594 (1994).

Banerjee, S., Turbulence structures, sixth P. V. Danckwerts memorial lecture. Chem. Eng. Sci., 47(8), 1793 (1991).

Beam, R. M., and Warming, R. F., An implicit factored scheme for the compressible Navier-Stokes equations. AIAA J. 16(4), 393 (1978). 
Beam, R. M., and Warming, R. F., An implicit finite difference algorithm for hyperbolic systems in conservation-law form. J. Comp. Phys. 22(1), 87 (1977).

Becker, A. A.. "The Boundary Element Method in Engineering: A Complete Course." McGraw-Hill. London. 1992.

Becker, S., Sokolichin. A.. and Eigenberger. G.. Gas-liquid foow in bubble colunns and loop reactors: Part II: Comparison of detailed experiments and flow simulation. Chem. Eng. Sci.. 49, 5747 (1994).

Beenackers, A. A. C. M. and van Swaaij, W. P. M., Review article number 42. Mass transfer in gasliquid slurry reactors. Chem. Eng. Sci., 48(18), 3109 (1993).

Bird. R. B.. Stewart. W. E.. and Lightlool, E. N.. "Transport Phenomenal." John Wiley \& Sons. New York. 1960.

Boger, D. V.. Viscoelastic flows through contractions, Ammi, Re': Fluid Mech. 19, 157 (1987).

Bouillard, J. X., Lyczkowski. R. W.. and Gidaspow. D.. Porosity distributions in a fluidized bed with an immersed obstacle. A/ChE. J. 35(6). 908 (1989).

Boussinesq, J., Essai sur la Théorie des Eaux Courantes. Mém. Prés. Acad. Sci. XXIII. 46. Paris. 1877.

Boysan. F., Ayers, W. H., and Swithenbank. J.. A fundamental mathematical modeling approach 10 cyclone design. Trans. I. Chem. E. 60, 222 (1982).

Brady. J. F., and Bossis. G., Stokesian dynamics. Ammu. Res: Fluid Mech. 20, 111 (1988).

Brereton, G.. and Korotney. D., Co-axial and oblique coalescence of two rising bubbles. In "Dynamics of Bubbles and Vortices Near a Free Surface." ASME. AMD 119, 1 (1991).

Bridgwater. J., Broadbent. C. J., and Parker. D. J.. Study of the influence of blade speed on the performance of a powder mixer using positron emmission particle tracking. Trans. l. Chem. E. 71(Part A), 675 (1993).

Briley, W. R., and McDonald, H. Solution of the three-dimensional compressible Navier-Stokes equations by an implicit technique. Proc. $4 \mathrm{th} \mathrm{Im}$. Cont. Nitm. Methods in Fluid Dynamics, Le'ture Notes in Physics, Springer-Verlag. Berlin. 1975. vol. 35. p. 105.

Brinkman. H. C., A calculation of the viscous force exerled by a flowing fluid on a dense swarm of particles. Appl. Sci. Res. A1, 27.81 (1947).

Brodkey. R. S.. and Lawelle, J., Reactor selectivity based on first order closures of the turbulent concentration equations. AIChE. J. 31, 111 (1985).

Canuto. C.. Hussaini, M. Y.. Quarteroni. A., and Zang. T. A.. "Spectral Methods in Fluid Dynamics." Springer-Verlag. Berlin, 1988.

Chang. S. L.. Lyczkowski. R. W., and Berry. G. F. Spectral dynamics of computer simulated iwodimensional fewtube fluidized beds. A/ChE. Simp. Ser: $26 \%$. 267 (1989).

Chaouki, J., Larachi, F. and Dudukovic. M. P. (eds.). "Non-Invasive Monitoring of Multiphase Flows," Elsevier, Amsterdam, 1997.

Chen. J. J. J.. Jamialahmadi. M. and Li. S. M. Effect of liguid depth on circulation in bubble columns: A visual study. Chem. Eng. Res. Des. 67. 2(1)3(1989).

Chen, R. C.. and Fan. L. S. Particle image velocimetry for characterizing the flow structures in threedimensional gas-liquid-solid fluidized beds. Che'm. Eng. Sci. 47. 3615 (1992).

Cheremisinoff, N. P., "Instrumentation for Complex Fluid Flows." Technomic Publishing Company. Lancaster. PA, 1986a.

Cheremisinofr. N. P., Review of experimental methods for studying the hydrodynamics of gith-solid fluidized beds, IdEEC Process Des. Devel. 25, 329)(19866).

Clift. R., An Occamist review of fluidized bed modelling. A/ChE. Simp. Se?: 296 89, 1 (1993).

Colenbrander. G. W. CFD research for the petrochemical industry. Appl. Sci. Res. 48, 211 (194)11.

Cook. T. L., Demuth, R. B., and Harlow, F. H., PIC calculations of multiphalse flow. J. Comp. Phis. 41 , $51(1981)$.

Corbett. A. M., Phillips. R. J., Kauten, R. J. and McCarthy. K. L., Magnetic resonance imaging of concentration and velocity profiles of pure fluids and solid suspensions in rotating geometries. J. Rheol. 39(5). 907 (1995). 
Correa, S. M., and Shyy, W., Computational models and methods for continuous gaseous turbulent combustion. Prog. Energy Combust. Sci. 13, 249 (1987).

Crochet, M. J., Numerical simulation of flow processes. Chem. Eng. Sci 42(5), 979 (1987).

Crowe, C. T., Review-Numerical models for dilute gas-particle flows. J. Fluids Eng. 104, 297 (1982).

Cundall, P. D., and Strack, O. D. L., A discrete numerical model for granular assemblies. Geotechnique 29, 47 (1979).

Curl, R. L., Dispersed phase mixing: I. Theory and effects in simple reactors. AIChE. J. 9(2), 175 (1963).

Cuvelier, C., Segal, A., and van Steenhoven, A. A., "Finite Element Methods and Navier-Stokes Equations." D. Reidel Publishing Company, Dordrecht, 1986.

Dahm, W., Southerland, L., and Su. L., Fully-resolved four dimensional spatio-temporal micromeasurements of the fine scale structure and dynamics of mixing in turbulent flows. AIChE Meet.. Miami Beach, FL, paper 262c (1995).

Delnoij, E., Lammers, F. A., Kuipers. J. A. M., and Van Swaaij, W. P. M., Dynamic simulation of dispersed gas-liquid two-phase flow using a discrete bubble model. Chem. Eng. Sci. 52, 1429 (1997a).

Delnoij, E., Kuipers. J. A. M.. and van Swaaij, W. P. M., Stimulation of bubble dynamics in gasliquid two-phase How using the volume of huid (VOF) method. Submitted for publication (1997b).

Derbyshire, J. A., Gibbs, S. J., Carpenter. T. A., and Hall, L. D., Rapid three-dimensional velocimetry by nuclear magnetic resonance imaging. AIChE. J. 40(8), 1404 (1994).

De Saegher. J. J.. Detemmerman. T., and Froment, G. F., Three dimensional simulation of high severity internally finned cracking coils for olefin production. Rev. Inst. Francais Du Pétrole $51(2)$. 245 (1996),

de Saint Venant, B., Note a Joindre un Mémoire sur la Dynamique des Fluides, Comptes Rendiss 17. $1240(1843)$.

Devanathan, N., Dudukovicz, M. P., Lapin, A., and Lübbert. A., Chaotic flow in bubble column reactors. Chem. Eng. Sci. 50, 2661 (1995).

Devanthan, N., Moslemian, D., and Dudukovicz. M. P., Flow mapping in bubble columns using CARPT. Chem. Eng. Sci. 45, 2285 (1990).

Ding, J., and Gidaspow. D.. A bubble fluidisation model using kinetic theory of granular flow. AIChE. J. 36(4), $523(1990)$.

Eggels, J. G. M., Direct and large eddy simulation of turbulent How in a cylindrical pipe geometry, Ph.D. Thesis. Delft University (1994).

Elgobashi, S., Particle-laden turbulent flows: Direct numerical simulation and closure models. Appl. Sci. Res. 48, 301 (1991).

Ettehadieh, B., Gidaspow. D., and Lyczkowski. R. W., Hydrodynamics of fluidization in al semicircular bed with a jet. A/ChE. J. 30(4), 529 (1984).

Fan, L. S., Bubble dynamics in liquid-solid suspensions. AIChE. Symp. Ser: 305 91, 1 (1995).

Fun, L. S.. "Gas-Liquid-Solid Fluidization Engineering," Butterworth Publishers. New York. 1989.

Farmer, W. M., Appl. Opt. 13,610(1974).

Farmer. W. M., Appl. Opt. 11, 2603 (1972).

Fletcher, C. A., "Computational Techniques for Fluid Dynamics, Vol. I. Fundamental and General Techniques. Springer Verlag, Berlin, 1988a.

Fletcher, C. A., "Computational Techniques for Fluid Dynamics, Vol. II, Specitic Techniques for Different Flow Categories." Springer Verlag, Berlin, 1988b.

Fox. R. O., Computational methods for turbulent reacting flows in the chemical process industry. Re: Inst. Francais Du Pétrole 51(2), 215 (1996).

Frank, M. J. W., Kuipers, J. A. M., Versteeg, G. F., and van Swaaij, W. P. M., Modelling of simultane- 
ous mass and heat transfer with chemical reactions using the Maxwell-Stefan theory-1. Model development and isothermal study. Chem. Eng. Sci. 50(10), 1645 (1995a).

Frank, M. J. W., Kuipers, J. A. M., Krishna. R., and van Swaaij, W. P. M., Modelling of simultaneous mass and heat transfer with chemical reactions using the Maxwell-Stefan theory-II. Nonisothermal study. Chem. Eng. Sci. 50(10), 1661 (1995b).

Gasche, H. E.. Edinger, C.. Kömpel, H.. and Hofmann, A fluid-dynamically based model of bubble column reactors. Chem. Eng. Technol. 13, 341 (1990).

Gaskey, S., Vacus. P., David. R.. André. J. C., and Villermaux, J., A method for the study of turbulent mixing using fluorescence spectroscopy. Exp. Fluids, 9, 137 (1990).

Ge, W., and Li. J., Pseudo-particle approach to hydrodynamics of gas-solid two-phase flow, Proc. CFB-5, Beijing. paper DT8 (1996).

Gharib, M., Hernan. M. A., Yavrouian. A. H., and Sarohia, V., Flow velocity measurement by image processing of optically activated tracers. AIAA paper no, 85-0172 (1985).

Gidaspow, D.. Hydrodynamics of fluidization and heat transfer: super computer modelling. Appl. Mech. Rev: 39(1), 1 (1986).

Gidaspow. D.. and Ettehadieh. B.. Fluidization in a tw')-dimensional bed with a jet. Part 2: Hydrodynamic modelling. Ind. Eng. Chem. Fundam. 22, 193 (1983).

Gidaspow, D., Lin, C., and Seo. Y. C.. Fluidization in a two-dimensional bed with a jel, Part 1: Experimental porosity distributions. Ind. Eng. Chem. Fundam. 22. 187 (1983).

Givi, P., and McMurtry, P. A.. Nonpremixed reaction in homogeneous turbulence: Direct numerical simulation. AIChE. J. 34, 1039 (1988).

Grace, J. R., Shapes and velocities of bubbles rising in infinite liquids. Trans. Inst. Chem. Eng. 51, 116 (1973).

Grace, J. R., Wairegi. T., and Nguygen. T. H.. Shapes and velocities of single drops and bubbles moving freely through immiscible liquids. Trans. Inst. Chem. Eng. 54, 167 (1976).

Grier, M. R.. and Fox. R. O.. A particle-conserving composition joint probability density function code. Report 259, Kansas State University, 1993.

Griffin. M. E., Diwaker, R., Anderson. J. D.. and Jones. E.. Computational fluid dynamics applied to flows in an internal combustion engine. AlAA 16th Aerospace Sciences Mect., paper 78-57. January 1978.

Guj. G. and De Matteis. G.. Fluid-particles interaction in particulate fluidized beds: Numerical and expermental analysis. Physico-Chemical Hydrodynamics 7(2/3), 145 (1986).

Hamill, I. S.. Hawkins, I. R.. Jones, I. P., Lo. S. M., Splawski. B. A.. and Fontenot, K.. The application of CFDS-FLOW3D to single and multiphase flow's in mixing vessels. AlChE Symp. Ser: 30591. $150(1995)$.

Hargreaves. J. H.. and Silvester. R. S.. Computational fluid dynamics applied to the analysis of deoiling hydrocyclone performance. Trans. I. Chem. E. 68, 365 (1990).

Harlow. F. H.. and Amsden. A. A.. Numerical calculation of multiphase fluid flow. J. Comp. Phys. 17, 19 (1975).

Harlow, F. H., and Amsden. A. A., KACHINA: An eulerian computer program for multifield fluid flows. Los Alamos Scientific Laboratory Report LA-5680. 1974.

Harris. C. K., Roekaerts. D.. and Rosendal. F. J. J.. Computational fluid dynamics for chemical reaction engineering. Chem. Eng. Sci. 51, 1569 (1995).

Hartge, E. U., Li. Y., and Werther. J.. Analysis of the local structure of two-phase flow in a fast fluidized bed. In "CFB Technology I" (P. Basu, ed.). Pergamon Press, 1986, p. 153.

Henline, W. D., Klein. H. H.. Scharff. M. F., and Srinivas, B., "Final report on computer modelling of the U-Gas Reactor," DOE. DE-ACO2-77E'T13406. Jaycor, 1981.

Hirsch, C., "Numerical Computation of Internal and External Flows. Vol. II: Computational Methods for Inviscid and Viscous Flows." Wiley, New York. 1990.

Hirsch, C., "Numerical Computation of Internal and External Flows. Vol, I: Fundamentals of Numerical Discretisation." Wiley. New York. 1988. 
Hirt, C. W., and Nichols, B. D., Volume of fluid (VOF) method for the dynamics of tree boundaries. J. Comp. Phys. 39, 201 (1981).

Hjertager, B. H., and Morud, K., Computational thuid dynamics simulation of bioreactors. In "Bioreactor Performance" (U. Mortensen and H. J. Noorman, eds.). Ideon, Lund, 1993. p. 47.

Hoffman, J. D. "Numerical Methods for Engineers and Scientists." McGraw-Hill. New York. 1992.

Hotfmann, A. C., and van den Bogaard. H. A., A numerical investigation of bubbles rising at intermediate Reynolds and large Weber numbers. Ind. Eng. Chem. Res. 34, 366 (1995).

Hoomans, B. P. B., Kuipers, J. A. M., and van Swaaij, W. P. M., Discrete particle simulation of a twodimensional gas-fluidised bed: Comparison between a soft sphere and a hard sphere approach. Submitted for publication (1998).

Hoomans, B. P. B., Kuipers, J. A. M., Briels, W. J., and van Swaaij, W. P. M.. Discrete particle simulation of bubble and slug formation in a two dimensional gas-Huidised bed: A hard sphere approach. Chem. Eng. Sci. 51(1), 99 (1996).

Hrenya, C. M., Bolio, E. J., Chakrabarti, D., and Sinclair. J. L., Comparison of low Reynolds k- $\epsilon$ turbulence models in predicting fully developed pipe flow. Chem. Eng. Sci. 50(12). 1923 (1995).

Hsieh, K. T., and Rajamani, R. K.. Mathematical model of the hydrocyclone based on physics of fluid How. AIChE. J. 37(5), $735(1991)$.

Huttenhuis, P. J. G., Kuipers. J. A. M., and W. P. M. van Swaaij, The effect of gals phase density on bubble formation at a single orifice in a two-dimensional gas-Huidized bed. Chem. Eng. Sci. 51(24), 5273 (1996).

Ichiki, K., and Hayakawa, H.. Dynamical simulation of fluidized beds: Hydrodynamicilly interacting granular particles. Phys, Rev E 52(1),658 (1995).

Ishii. M., "Thermo-Fluid Dynamic Theory of Two-Phase Flow," Eyrolles. Paris, 1975.

Jackson. R., Progress toward a mechanics of dense suspensions of solid particles. AlChE. Symp. Ser: $30190,1(1994)$.

Jameson, A., Computational aerodynamics tor aircraft design. Science 245, 361 (1988).

Johansen, S. T., and Kolbeinsen. L.. "Applications of Computational Fluid Dynamics in Optimisation and Design of Metallurgical Processes," SINTEF Materials Technology, N-703+ TrondheimNTH, Norway, 1996.

Joshi, J. B. and Sharma, M. M.. A circulation cell model for bubble columns. Trans. Inst. Chem. Engrs. 57, 244 (1979).

Kawaguchi, T., Yamamoto, Y., Tanaka, T., and Tsuji, Y.. Numerical simulation of a single rising bubble in a two-dimensional fluidized bed. Proc. 2nd Int. Conf. Multiphase Flow. Kyoto/Japan. FB2-17-FB2-22 (1995).

Keunings, R.. Progress and challenges in computational theology. Rheol. Acta 29, 556 (1990).

Keunings. R, and Crochet, M. J., Numerical simulation of the flow of a viscoelastic fluid through an abrupt contraction. J. Non-Newtonian Fluid Mech. 14, 279 (1984).

Kim, J., Moin, P., and Moser, R. D.. Turbulence Statistics in fully-developed channel flow at low Reynolds number. J. Fluid Mech. 177, 133 (1987).

Koelman, J. M. V. A., and Hoogerbrugge. P. J., Dynamic simulations of hard-sphere suspensions under steady shear. EuroPhys. Lett 21(3), 363 (1993).

Kothe, D. B., Mjolsness, R. C.. and Torrey, M. D., "RIPPLE: A Computer Program for Incompressible Flows with Free Surfaces," Los Alamos Scientific Laboratory Report LA-12007-iMS, 1991.

Kresta, S. M., and Wood, P. E.. Prediction of the three-dimensional turbulent flow in stirred tanks. AIChE. J. 37(3), 448 (1991).

Kuipers. J. A. M., A two-fluid micro balance model of fluidized beds. Ph.D. Thesis, University of Twente (1990).

Kuipers, J. A. M., and van Swaaij, W. P. M., Developing flow in CFB-risers: A computational study of the effect of riser inlet configuration on How structure development. Workshop II on Modelling and Control of Fluidized Bed Systems, CFB-5, Beijing (1996).

Kuipers, J. A. M., van Duin, K. J., van Beckum, F. P. H.. and van Swaaij, W. P. M.. Computer simula- 
tion of the hydrodynamics of a two-dimensional gas-fluidized bed. Comp. Chem. Eng. 17(8), 839 (1993).

Kuipers, J. A. M., van Duin. K. J., van Beckum. F. P. H., and van Swaaij. W. P. M.. A numerical model of gas-fluidized beds. Chem. Eng. Sci, 47(8), 1913 (1992a).

Kuipers. J. A. M., Tammes, H., Prins, W., and van Swaaij, W. P. M., Expermental and theoretical porosity profiles in a two-dimensional gas-fluidized bed with a central jet. Powder Techlmol. 71 , $87(1992 \mathrm{~b})$.

Kuipers, J. A. M., Prins, W., and van Swaaj,. W. P. M., Numerical calculation of wall-lo-bed heal transfer coefficients in gas-fluidized beds. AIChE. J. 38(7), 1079 (1992c).

Kuipers, J. A. M., Prins, W. and van Swaaij, W. P. M., Theoretical and experimental bubble formition at a single orifice in a twomdimensional gas-fluidized bed. Chem. Eng. Sci. 460111, 2881 (1991).

Kumar, S., VanderHeyden, W. B., Devanathan, N., Padial, N. T., Duclukovicz. M. P., and Kashiwa. B. A., Numerical simulation and experimental verification of the gats-liquid flow in bubble columns. A/ChE Symp. Ser 30591,11 (1995).

Kunii. D., and Levenspiel. O., "Fluidization Engineering," 2nd ed. Butterworth-Heinemann. Boston. 1991.

Lapin. A., and Lubbert. A., Numerical simulations of the dynamics of two-phase gas-liquid fows in bubble columns. Chem. Eng. Sci. 49, 3661 (1994).

Larachi. F., Cassanello, M., Chaouki. J., and Guy, C., Flow structure of the solids in a 3-D gas-liculudsolid fluidized bed, AIChE.J. 42(9), 2439 (1996).

Larachi, F., Chaouki. J., and Kennedy. G., 3-D mapping of solids flow tields in multiphase reactors with RPT. AIChE. J. 41(2), 439 (1995).

Launder, B. E., Current capabilities for modelling turbulence in industrial flows. Appl. Sci. Res. 48 , 247 (1991).

Lemoine. F. Wolff, M.. and Lebouche, M., Simultaneous concentration and velocity meatsurement us. ing combined laser-induced fluorescence and laser Doppler velocimetry: Application to turbulent transport. Exp Fluids 20, 319 (1996).

Leonard, A. D.. and Hill, J. C., Direct numerical simulation of turbulent flows with chemical reaction. J. Sci. Comp. 3, 25 (1988).

Levenspiel. O.. "Chemical Reaction Engineering." John Wiley \& Sons. New' York, 1962

Li. K. T., and Toor, H. L., Turbulent renctive mixing with a series-parallel reaction: Eflect of mixing on yield. $A / C h E . J .32,1312(1986)$.

Lim. K. S.. Zhu. J. X., and Grace, J. R., Hydrodynamics of gats-solid fluidization. Imt. I. Maltiphase' Flow 21, 141 (1995).

Lin. T. J., Reese, J., Hong, T., and Fan, L. S., Quantitative analysis and computalion of iwodimensional bubble columns. AIChE. J. 42(2). 301 (1996).

Lyons, S. L.. Hanratty, T. J., and McLaughlin. J. B.. Turbulence-producing eddies in the viscous wall region. AIChE. I. 35(12), 1962 (1989).

Mampaey, F.. and Xu, Z. A., An experimental and simulation study of nould filling conbined with heat transfer. In "Computational Fluid Dynamics '92, (C. Hirsch. J. Periaux and W. Kordulla. eds.), Elsevier. Amsterdam, 1992, Vol, 1, 421.

Manger, E., Modelling and simulation of gas-solids flow in curvilinear coordinates. Ph.D. Thesis. Telemark College, 1996.

Marcum, D. L., and Hoffman, J. D., Calculation of three-dimensional inviscid flow-fields in propulsive nozzles with centerbodies. Am. Inst. Aeronatics Astronautics. Propulsion Power 4(2). 172 (1988).

Martin, M. P.. Turlier, P., and Bertrand, J. R.. Gas and solid behavior in cracking circulating fluidized beds. Powder Techol. 70, 249 (1992).

Matsunaga, K., Mijata, H., Aoki, K., and Zhu, M., Finite-difference simulation of 3D vortical flows past road vehicles. Vehicle Hydrodynamics, SAE Special Publication 908. 65 (1992). 
McMurtry, P. A.. and Givi, P.. Direct numerical simulations of mixing and reaction in a nonpremixed homogeneous turbulent flow. Combust. Flame 77, 171 (1989).

Melli, T. R., and Scriven. L. E., Theory of two-phase cocurrent downflow in networks of passages. Ind. Eng. Chem. Re's. 30, 951 (1991).

Merzkirch, W., "Flow Visualization." Academic Press, Orlando, 1987.

Mompean, G., and Deville, M. O., Recent developments in three-dimensional unsteady flows of nonNewtonian fluids. Rev. Inst. Francais Du Pétrole 51(2), 261 (1996).

Myong, H. K.. and Kasagi, N., A new approach to the improvement of k- $\epsilon$ models for wall-bounded shear flows. JSME Int. J. (Series II) 33, 63 (1990).

Nakayama. T., and Mori. M., An Eulerian finite element method for time-dependent free surface problems in hydrodynamics. Int. J. Num. Methods Fluids 22, 175 (1996).

Navier. M., Mémoire sur les Lois du Mouvement des Fluides. Mem. de l'Acad. de Sci. 6, 389 (1827)

Nichols, B. D. Hirt. C. W., and Hotchkiss, R. S., "SOLA-VOF: A Solution Algorithm for Transient Fluid Flow with Multiple Free Boundaries," Los Alamos Scientific Laboratory Report LA-8355. 1980.

Nieuwland, J. J., Hydrodynamic modelling of gas-solid two-phase flows. Ph.D. Thesis, University of Twente, 1995.

Nieuwland, J. J., Veenendaal. M. L., Kuipers. J. A. M., and van Swaaij, W. P. M., Bubble formation at a single oritice in a two-dimensional gas-fluidized bed. Chem. Eng. Sci. 51(17), 4087 (1996a).

Nieuwland. J. J., Sing Annaland van. M., Kuipers, J. A. M., and van Swaaij, W. P. M., Hydrodynamic modelling of gas-particle flows in riser reactors. AIChE. J. 42(6), 1569 (1996b).

Nieuwland, J. J.. Kuipers, J. A. M., and van Swataij, W. P. M., Theoretical and experimental study of CFB riser hydrodynamics. Proc. CFB-5, Beijing, paper MSS3 (1996c).

Nieuwland. J. J., Meijer. R., Kuipers. J. A. M., and van Swaaij, W. P. M., Measurement of solids concentration and axial solids velocity in gas-solid two-phase flows. Powder Technol. 87, 127 (1996d).

Nieuwland. J. J.. Kuipers, J. A. M., and van Swaaij, W. P. M., Bubble formation in a two-dimensional gas-fluidized bed at elevated pressures, Proc. Fluidization VIII, Tours (1995).

Ohta. M., Yamamoto, M., and Suzuki. M., Numerical analysis of a single drop formation process under pressure pulse condition. Chem. Eng. Sci. 50(18), 2923 (1995).

Ottino, J. M. Mixing and chemical reactions-A tutorial. Chem. Eng. Sci, 49(24A), 4005 (1994).

Pain. Y., and Banerjee, S., Numerical simulation of particle interactions with wall turbulence. Phys. Fluids 8(10), 2733 (1996a).

Pan, Y., and Banerjee, S., Numerical investigation of the elfects of large particles on wall-turbulence. Submitted for publication (1996b).

Patankar. S. V., "Numerical Heat Transfer and Fluid Flow," McGraw-Hill, New York, 1980.

Pedinotti, S., Mariotti, G.. and Banerjee, S., Effect of Reynolds number on particle behavior near walls in two phase turbulent flows. Proc. 5th. Int. Symp. Flow Mod. Turb. Meas., Paris, Presses Pont et Chausees, 425 (1993).

Pedinotti, S.. Mariotti, G.. and Bamerjee, S., Direct numerical simulation of particle behavior in the wall region of turbulent lows in horizontal channels. Int. J. Mult. Flow 18, 927 (1992).

Perng, C. Y., and Murthy, J. Y., A moving-delorming-mesh technique for simulation of flow in mixing lanks. AlChE. Symp. Ser: 293 89, 37 (1992).

Pipino, M., and Fox, R., Reactive mixing in a tubular jet reactor: A comparison of PDF simulations with experimental data. Chem. Eng. Sci. 49(24B), 5229 (1994).

Pita. J. A., and Sundaresan, S., Developing tlow of a gas-particle mixture in a vertical riser. $A / C h E, /$, 39(4). 541 (1993).

Pita, J. A., and Sundaresan. S., Gas solid tlow in vertical tubes. AlChE. J. 37(7), 1009 (1991).

Poisson, S. D. Mémoire sur les Equations Générales de l'Equilibre et du Mouvement des Corps Solides Elastiques et des Fluides. J. de L'Ecole Polytechn. 13, 139 (1831). 
Pope, S. B.. Lagrangian PDF methods for turbulent flows. Annu. Rev. Fluid Mech. 26, 23 (1994). Pritchett. J. W., Blake, T. R., and Garg, S. K., A numerical model of gas fluidized beds. AIChE. Symp. Ser: $176,74,134$ (1978).

Ranade, V. V., and Joshi, J. B., Flow generated by a disc turbine: Part I. Experimental. Trans. I. Chem. E. 68(Part A). 19 (1990a).

Ranade, V. V., and Joshi, J. B., Flow generated by a disc turbine: Part II. Mathematical modelling and comparison with experimental data. Trans. I. Chem. E. 68(Part A), 34 (1990b).

Reese, J.. and Fan, L. S.. Transient flow structure in the entrance region of a bubble column using particle image velocimetry. Chem. Eng. Sci. 49(24B), 5623 (1994).

Reynolds. W. C., The potential and limitations of direct and large eddy simulations. In "Whither Turbulence"? Turbulence at the Crossroads" (J. L. Lumley, ed.). Springer Verlag, Berlin, 1991, p. 313.

Rivard. W' C.. and Torrey, M. D.. "THREED: An Extension of the K-FIX Code for Three-Dimensions. "Los Alamos Scientific Laboratory Report AL-NUREG-6623. 1979.

Rivard. W. C., and Torrey, M. D., "K-FIX: A Computer Program for Transient, Two-DImensional, Two-Fluid Flow," Los Alamos Scientific Laboratory Report LA-NUREG-6623, 1977.

Roache. P. J., "Computational Fluid Mechanics," Hermosa, Albuquerque, NM. 1972.

Rodi. W.. "Turbulence Models and Their Application in Hydraulics-A State of the Art Review." International Association for Hydraulic Research Section on Fundm. of Division II: Exp. and Math. Fluid Dynamics.

Rogallo. R. S., and Moin, P., Numerical simulation of turbulent flows. Ammu. Rev: Fluid Mech. 16, 99 (1984).

Rowe. P. N.. In "Fluidization" (J. F. Davison and D. Harrison. eds.). Academic Press, London, 1971.

Rudman, M., Volume-tracking methods for interfacial flow calculations. Int. J. Num. Methods Fluids $24,671(1997)$.

Ryskin, G.. and Leal, L. G.. Numerical solution of free-boundary problems in fluid mechanics. Part I. The finite difference technique. J. Fluid Mech. 148, 1 (1984a).

Ryskin. G.. and Leal, L. G.. Numerical solution of free-boundary problems in fluid mechanics. Part II, Buoyancy-driven motion of a gas bubble through a quiescent liquid. J. Fluid Mech. 148, 19 (1984b).

Ryskin. G., and Leal. L. G.. Numerical solution of free-boundary problems in fluid mechanics. Par III. Bubble deformation in an axisymmetric straining fow. J. Fluid Mech. 148, 37 (1984c).

Scharff, M. F., Chan, R. K-C.. Chiou. M. J., Dietrich. D. E.. Dion, D. D.. Klein, H. H., Laird, D. N.. Levine, H. R.. Meister, C. A.. and Srinivas, B., "Computer Modelling of Mixing and Agglomeration in Coal Conversion Reactors," Vols. I \& Il, DOE/ET/10329-1211, Jaycor, 1982.

Schlichting. H., "Boundary Layer Theory." McGraw-Hill. New York, 1975.

Schneyer. G. P.. Peterson. E. W.. Chen, P. J., Brownell. D. H., and Blake. T. R., "Computer Modelling of Coal Gasification Reactors. "Final Report for June 1975-1980 DOE/ET/10247. Systems, Science and Software, 1981.

Shaw, C. T., "Predicting Vehicle Aerodynamics Using Computational Fluid Dynamics-A User's Perspective." Research in Automotive Aerodynamics. SAE Special Publication 747, Feb. 1988, p. 119.

Simonin. O.. Modelling turbulent reactive dispersed two-phase flows in industrial equipments. Proc: Third World Conf. Applied Computational Fluid Dynamics, May 19-23, Freiburg, Germany. Workshop E, 17.9, 1996.

Sinclair. J.. and Jackson, R., Gas-particle flow in a vertical pipe with particle-particle interactions. AIChE. J. 35(5), $1473(1989)$.

Sokolichin. A., and Eigenberger. G., Gas-liquid flow in bubble columns and loop reactors: Part I: Detailed modelling and numerical simulation. Chem. Eng. Sci. 49, 5735 (1994).

Sokolichin, A., Eigenberger, G., Lapin, A., and Lübbert. A., Dynamic simulation of gas-liquid twophase flows-Euler/Euler versus Euler/Lagrange. Chom. Eng. Sci. 52, 611 (1997). 
Spielman, L. A., and Levenspiel, O.. A Monte-Carlo treatment for reacting and coalescing dispersed phase systems. Chem. Eng. Sci. 20, 247 (1965).

Squires, K. D., and Eaton. J. K., Particle response and turbulence modification in isotropic turbulence. Phys, Fluids A2, 1191 (1990).

Steijsiger, C., Lankhorst. C. M., and Roman, Y. R., Influence of gas phase reactions of the deposition rate of silicon carbide from the precursors methyltrichlorosilane and hydrogen. In "Numerical Methods in Engineering '92" (C. Hirsch and O. C. Zienkiewicz, eds.). Elsevier, Amsterdam, 1992, p. 857.

Stewart. H. B., and Wendrolf, B.. Two-phase flow: Models and methods. J. Comp. Phys. 56, 363 (1984).

Stokes, G. G. On the theories of internal friction of fluids in motion. Trans. Cambr: Phil. Soc. 8, 287 (1845).

Svendsen, H. F., Jakobsen. H. A.. and Torvik. R.. Local how structure in internal loop and bubble column reactors. Chem. Eng. Sci. 47(13/14), 3297 (1992).

Syamlal, M., and Gidaspow. D.. Hydrodynamics of fluidization: Prediction of wall-to-bed heat transfer coefficients. A/ChE. J. 31(1). 127 (1985).

Tanaka, T., Yonemura. S.. Kiribayashi, K., and Tsuji. Y., Cluster formation and particle-induced instability in gas-solid two-phase hows predicted by the DSMIC method. JSME. Ser. B 39(2), 239 (1996).

Targett, M. J., Retallick. W. B., and Churchill. S. W.. Flow through eurved rectangular channels of large aspect ratio. AIChE. J. 4115), 1061 (1995).

Tennekes. H., and Lumley, J. L.. "A First Course in Turbulence." The MIT Press, Cambridge, MA. 1977.

Theologos, K. N., and Markatos. V. C.. Modelling of flow and heat transfer in fluidized catalytic cracking riser-type reactors. Trans. I. Chem. E. 70(Part A), 239 (1992).

Thompson, J. F., ed., "Numerical Grid Generation." North-Holland, New York, 1982.

Thompson. J. F., Warsi. Z. U. A.. and Mastin. C. W.. "Numerical Grid Generation," Foundations and Applications." North-Holland. New York. 1985.

Thompson, J. F., Warsi. Z. U. A.. and Mastin. C. W.. Boundary-fitted coordinate systems for numerical solution of partial differential equations-A review. J. Comp. Phys. 47, 1 (1982).

Tomiyama, A., Zun. 1.. Sou, A., and Sakaguchi. T., Numerical analysis of bubble motion with the VOF method. Nucl. Eng. Des. 141, 69 (1993).

Torvik, R., and Svendsen, H. F., Modelling of slurry reactors, a fundamental approach. Chem. Eng. Sci 45, 2325 (1990).

Toye, D., Marchot, P.. Crine, M.. and L'Homme. G.. Analaysis of liquid fiow distribution in trickling How reactor using computer assisted x-ray tomography. Trans. I. Chem. E. 73(Part A), 258 (1995).

Trambouze, P., Computational fluid dynamics applied to chemical reaction engineering. Rev. Inst. Francais du Petrole 48(6). 595 (1993).

Trapp, J. A., and Mortensen. G. A.. A discrete particle model for bubble slug two-phase flow. J. Comp. Phys. 107, 367 (1993).

Tsai, K., and Fox, R. O., PDF simulations of a turbulent series-parallel reaction in an axisymmetric reactor. Chem. Eng. Sci. 49(24B), $5141(1994)$.

Tsuji, Y., Kawaguchi. T., and Tanaka. T., Discrete particle simulation of two-dimensional fluidized bed. Powder Technol 77, 79 (1993).

Tsuo, Y. P., and Gidaspow, D., Computation of flow patterns in circulating fluidized beds. AlChE. J. 36(6), $885(1990)$.

Udaykumar, H. S., Shyy, W., and Rao, M. M.. ELAFINT: A mixed Eulerian-Lagrangian method for thuid flows with complex and moving boundaries. Int. J. Num. Methods Fluids 22, 691 (1996).

Van Kemenade, V.. and Deville. M. O.. Spectral elements for viscoelastic flows with change of type. J. Rheol. 38(2), 291 (1994). 
Versteeg, G. F., Kuipers, J. A. M., van Beckum, F. P. H., and van Swaaij, W. P. M., Mass transfer with complex reversible chemical reactioms-II. Parallel reversible chemical reactions. Chem. Eng. Sci. 45(1), $183(1990)$.

Versteeg, G. F.. Kuipers, J. A. M., van Beckum, F. P. H., and van Swaaij, W. P. M., Mass transfer with complex reversible chemical reactions-I. Single reversible chemical reaction. Chem. Eng. Sci. 44(10). 2295(1989).

Villermaux, J., Personal Communication, 1996.

Villermaux, J., and Devillon. J. C., Représentation de la Coalescence el de la Redispersion des Domaines de Ségrégation dans un Fluide par un Modele d'Interaction Phénomenologique. Proc. 2nd lnt. Symp. Chem. React Engin., Amsterdam, B 1-B 13 (1972).

Villermaux. J., and Falk. L.. Recent advances in modelling micromixing and chemical reaction. Rev: Inst. Francais Du Pétrole 51(2), 205 (1996).

Villermaux. J., and Falk, L.. A generalized mixing model for initial contacting of reactive fluids. Chem. Eng. Sci. 49(24B), 5127 (1994).

Vortmeyer. D., and Schuster, J., Evaluation of steady flow profiles in rectangular and circular packed beds by a variational method. Chem. Eng. Sci. 1691 (1983).

Wang. J. W.. and Andrews. J. R. G., Numerical simulation of flow in helical ducts. $A I C h E$. J. 41(5). 1071 (1995).

Wang. B. N.. and Hoffman, J. D., Algebraic grid generation for annular nozzle flowfield prediction. In "Proceedings of the First International Conference on Numerical Grid Generation in Computational Fluid Dynamics," Pineridge Press, Swansea, United Kingdom, 399 (1986).

Warsi. Z. U. A.. "Fluid Dynamics, Theoretical and Computational Approaches." CRC Press, Boca Raton, FL (1993).

Welch, J. E.. Harlow. F. H.. Shannon, J. P., and Daly, B. J., "The MAC Method: A Computing Technique for Solving Viscous Incompressible Transient Fluid Flow Problems Involving Free Surfaces." Los Alamos Scientific Laboratory Report LA-3425, 1965.

Wen-Jei Yang, "Handbook of Flow Visualization." Hemisphere Publishing Corporation, New York. 1989.

Westerhout, R. W. J., Kuipers. J. A. M. and van Swaiaj. W. P. M., Development, Modelling and Evaluation of a (Laminar) Entrained Flow Reactor for the Determination of the Pyrolysis Kinetics of Polymers. Chem. Engng. Sci., 51(10), 2221, (1996).

Westerweel. J.. Digital particle image velocimetry-theory and application. Ph.D. Thesis, Delft University. 1993.

Xu. Q.. and Michaelides, E. E.. A numerical study of the flow over ellipsoidal objects inside a cylindrical tube. Int. J. Num. Methods Fluids 22, 1075 (1996).

Yoo, J. Y., and Na. Y.. A numerical study of the planar contraction flow of a viscoelastic fluid using the SIMPLER algorithm. I. Non-Newtonian Fluid Mech. 39, 89 (1991).

Youngs, D. L.. Time-dependent multi-material flow with large fluid distortion. In "Numerical Methods for Fluid Dynamics" (K. W. Morton and M. J. Baines, eds.). Academic Press, New York. 1982, p. 273.

Zienkiewicz. O.C., and Taylor, R. L.. "The Finite Element Method, Basic Formulation and Linear Problems." 4th ed. McGraw Hill. London, 1989a. Vol. I.

Zienkiewicz, O. C.. and Taylor, R. L.. "The Finite Element Method, Solid and Fluid Mechanics, Dynamics and Non-Linearity," 4th ed.. McGraw Hill, London, 1989b, Vol. II. 